Universidad Autónoma de MADRID

Facultad de Ciencias

Departamento de Física Teórica

\title{
The Higgs as a pseudo-Goldstone boson
}

Memoria de Tesis Doctoral realizada por

Sara Saa Espina

y presentada ante el Departamento de Física Teórica

de la Universidad Autónoma de Madrid

para la obtención del Título de Doctora en Física Teórica.

Tesis Doctoral dirigida por

M. Belén Gavela Legazpi,

Catedrática del Departamento de Física Teórica

de la Universidad Autónoma de Madrid.

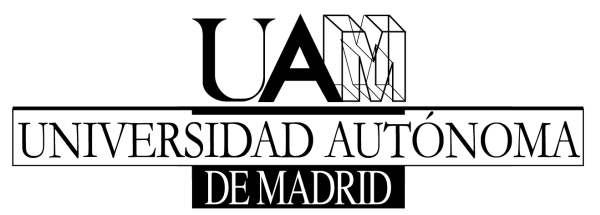

ift

Madrid, 27 de junio de 2017 


\section{Contents}

$\begin{array}{lr}\text { Purpose and motivation } & 1\end{array}$

$\begin{array}{ll}\text { Objetivo y motivación } & 4\end{array}$

I Foundations $\quad 9$

1 The Standard Model (SM) 11

$\begin{array}{ll}1.1 \text { Gauge fields and fermions } & 11\end{array}$

$\begin{array}{lll}1.2 & \text { Renormalizability and unitarity } & 14\end{array}$

$2 \quad$ Symmetry and spontaneous breaking $\quad 17$

$\begin{array}{lll}2.1 & \text { Symmetry types and breakings } & 17\end{array}$

$\begin{array}{ll}2.2 & \text { Spontaneous breaking of a global symmetry } \\ & 18\end{array}$

2.2.1 Goldstone theorem 20

2.2.2 Spontaneous breaking of the chiral symmetry in QCD 21

$\begin{array}{lll}2.3 & \text { Spontaneous breaking of a gauge symmetry } 26\end{array}$

3 The Higgs of the SM $\quad 29$

3.1 The EWSB mechanism 29

3.2 SM Higgs boson phenomenology at the LHC: production and decay 32

3.3 The Higgs boson from experiment 34

$\begin{array}{ll}3.4 & \text { Triviality and Stability } \\ \end{array}$

4 Having a light Higgs $\quad 41$

4.1 Naturalness and the "Hierarchy Problem" 41

4.2 Higgsless EWSB 44

$\begin{array}{lll}4.3 & \text { The Higgs as a pseudo-GB } & 45\end{array}$

II Higgs Effective Field Theory (HEFT) 49

$5 \quad$ Effective Lagrangians $\quad \mathbf{5 1}$

5.1 Generalities of EFTs $\quad 51$

5.2 The Chiral Effective Lagrangian $\quad 52$

5.3 Including a light Higgs 54

$\begin{array}{lll}5.4 & \text { Linear vs. Non-linear approach } & 56\end{array}$

6 Renormalization of the scalar sector $\quad 59$

$\begin{array}{lll}6.1 & \text { Renormalization in EFTs } & 59\end{array}$ 
6.2 The scalar Lagrangian 60

6.2.1 Parametrization of the GB matrix 62

6.2.2 Counterterm Lagrangian 64

6.3 Renormalization of the off-shell Green functions 66

$\begin{array}{lll}6.3 .1 & \text { 1-point functions } & 67\end{array}$

$\begin{array}{lll}6.3 .2 & \text { 2-point functions } & 67\end{array}$

$\begin{array}{lll}\text { 6.3.3 3-point functions } & 69\end{array}$

$\begin{array}{lll}6.3 .4 & 4 \text {-point functions } & 72\end{array}$

6.3.5 Comparison with previous works and SM limit $\quad 75$

6.3.6 Dealing with the apparent non-invariant divergences (NIDs) 76

$\begin{array}{lll}6.4 & \text { Renormalization Group Equations } & 78\end{array}$

7 Complete renormalization $\quad \mathbf{8 1}$

7.1 A gauge and Yukawa theory for a manifold of scalars 81

7.1.1 One-loop renormalization $\quad 83$

$\begin{array}{lll}7.2 & \text { One-loop HEFT } & 85\end{array}$

7.2.1 Renormalization of the Leading Lagrangian 88

7.2.2 Renormalization of the Sub-leading Lagrangian 90

\section{Linear sigma model for a pseudo-Goldstone Higgs 95}

8 Presentation of the model $\quad 97$

$\begin{array}{lll}8.1 & \text { The } S O(5) / S O(4) \text { scalar sector } & 98\end{array}$

$\begin{array}{lll}8.1 .1 & \text { The scalar potential } & 99\end{array}$

$\begin{array}{ll}\text { 8.1.2 Scalar-gauge boson couplings } & 102\end{array}$

8.1.3 Renormalization and scalar tree-level decays 102

$\begin{array}{ll}\text { 8.1.4 Scalar parameter space } & 103\end{array}$

$\begin{array}{lll}8.2 & \text { Fermionic sector } & 106\end{array}$

8.2.1 The fermionic Lagrangian 108

$\begin{array}{lll}8.3 & \text { Coleman-Weinberg potential } & 110\end{array}$

9 Phenomenology $\quad \mathbf{1 1 3}$

9.1 Bound from Higgs measurements 113

$\begin{array}{lll}9.2 & \text { Precision electroweak constraints } & 114\end{array}$

$\begin{array}{lll}9.2 .1 & S, T \text { and } g_{L}^{b} & 114\end{array}$

9.2.2 Scalar contributions in the linear $S O(5)$ model: $h$ and $\sigma \quad 115$

$\begin{array}{lll}9.2 .3 & \text { Fermionic contributions } & 117\end{array}$

$\begin{array}{lll}9.3 & \text { Higgs and } \sigma \text { coupling to gluons } & 122\end{array}$

$\begin{array}{lll}\text { 9.3.1 } & \boldsymbol{h} \leftrightarrow \boldsymbol{g g} \text { transitions } & 123\end{array}$

9.3.2 $\boldsymbol{\sigma} \leftrightarrow \boldsymbol{g} \boldsymbol{g}$ transitions $\quad 124$

9.4 Higgs and $\sigma$ decay into $\gamma \gamma \quad 126$

$\begin{array}{lll}9.4 .1 & h \leftrightarrow \gamma \gamma \text { transitions } & 126\end{array}$

$\begin{array}{lll}9.4 .2 & \sigma \leftrightarrow \gamma \gamma \text { transitions } & 128\end{array}$

$\begin{array}{lll}9.5 & \text { The } \sigma \text { resonance at the } \mathrm{LHC} & 129\end{array}$

10 Connection with EFTs

$\begin{array}{ll}10.1 \text { Model independent analysis } & 134\end{array}$ 
10.1.1 Impact on Higgs observables $\quad 140$

$\begin{array}{lll}10.2 & \text { Explicit fermion sector } & 143\end{array}$

10.2.1 Integrating out only the heavy fermions 144

$\begin{array}{ll}\text { 10.2.2 Integrating out only the heavy scalar } & 146\end{array}$

$\begin{array}{ll}\text { 10.2.3 Combining the two limits } & 147\end{array}$

IV HEFT and Baryon Number Violation 149

11 Baryon non-invariant couplings in Higgs effective field theory $\quad 151$

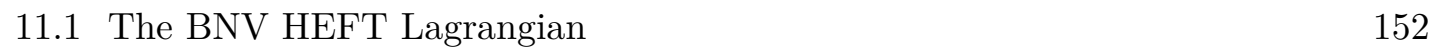

$\begin{array}{ll}11.2 \text { Comparison with the SMEFT } & 153\end{array}$

$\begin{array}{lll}11.3 & \text { Flavor contraction counting } & 155\end{array}$

$\begin{array}{lr}\text { Summary and conclusions } & 157\end{array}$

$\begin{array}{ll}\text { Resumen y conclusiones } & 161\end{array}$

$\begin{array}{ll}\text { Bibliography } & 167\end{array}$ 


\section{List of Figures}

$\begin{array}{lll}1.1 & \text { Particle content of the SM } & 12\end{array}$

2.1 Spontaneous symmetry breaking in a ferromagnet 18

$\begin{array}{lll}2.2 & \text { Paraboloid versus Mexican hat potentials } & 19\end{array}$

3.1 Diagrams contributing to WW scattering 32

3.2 Local p-value as a function of the Higgs mass observed in the CMS and ATLAS experiments 32

3.3 Computed SM Higgs production cross sections and branching ratios 33

3.4 Higgs production channels at LHC 33

$\begin{array}{ll}3.5 & \text { Higgs decay channel into photons } \\ 34\end{array}$

3.6 ATLAS and CMS best fits for the signal strengths 36

3.7 ATLAS and CMS combined fit of $\kappa_{g}$ vs $\kappa_{\gamma}$. 37

3.8 ATLAS and CMS combined measurements of coupling modifiers. $\quad 38$

3.9 Fit for Higgs couplings as a function of the particle mass. 39

3.10 Regions for the stability of the SM vacuum in terms of Higgs and top pole masses. 40

$\begin{array}{lll}4.1 & \text { Relaxion potential } & 43\end{array}$

6.1 Diagram contributing to the Higgs 1-point function. 67

$\begin{array}{ll}6.2 & \text { Diagrams contributing to the } \pi \text { self-energy. }\end{array}$

$\begin{array}{ll}\text { 6.3 Diagrams contributing to the Higgs self-energy. } & 69\end{array}$

6.4 Diagrams contributing to the $h h \rightarrow h$ amplitude, not including diagrams obtained by crossing. $\quad 70$

6.5 Diagrams contributing to the $\pi \pi \rightarrow h$ scattering amplitude, not including diagrams obtained by crossing. $\quad 71$

6.6 Diagrams contributing to the $h h \rightarrow h h$ amplitude, not including diagrams obtained by crossing. $\quad 73$

6.7 Diagrams contributing to the $\pi \pi \rightarrow h h$ amplitude, not including diagrams obtained by crossing.

6.8 Diagrams contributing to the $\pi \pi \rightarrow \pi \pi$ amplitude, not including diagrams obtained by crossing. $\quad 75$

8.1 Schematics of the $S O(5) \rightarrow S O(4)$ model $\quad 97$

$\begin{array}{lll}8.2 & \text { Parameter space for } m_{\sigma} \text { and } \sin ^{2} \gamma \text {. } & 105\end{array}$

$\begin{array}{lll}8.3 & \text { Schematics of light fermion mass generation } & 107\end{array}$

9.1 Uncombined contributions of the scalar sector (black curve) and the exotic fermionic sector to the parameters $S$ and $T$. 
9.2 Combined contributions to $S$ and $T$ from the scalar sector and the exotic fermionic sector.

9.3 Scalar and fermionic impact on the $T$ parameter and on the $Z-b_{L}-b_{L}$ coupling $g_{b}^{L}$.

9.4 Examples of correlations between fermion mixings and EW precision measurements.

9.5 LHC constraints on $\sin ^{2} \gamma$ versus $\sigma$ mass parameter space neglecting exotic heavy fermion contributions.

9.6 LHC constraints on $\sin ^{2} \gamma$ versus $\sigma$ mass parameter space considering non-negligible exotic heavy fermion contributions.

10.1 Schematic fermion mass operator at low scales with arbitrary insertions of the scalar fields. 


\section{List of Tables}

$\begin{array}{lll}1.1 & \text { Transformation properties of the SM fermions } & 13\end{array}$

6.1 Operators in the $\mathcal{L}_{4}$ Lagrangian for the Higgs and GBs. 63

6.2 Counterterms from the non-linear Lagrangian (only $h$ and GBs) contributing to Green functions 67

8.1 Heavy fermion charges assignments under the different groups. 108

10.1 Yukawa effective interactions for different fermion embeddings in $S O(5) 135$

10.2 Effective operators and first corrections in $1 / \lambda \quad 139$

10.3 Table with the definitions for the renormalization factors. 144

10.4 Leading order effective operators with heavy fermions integrated out. 145

$\begin{array}{ll}10.5 \text { Effective operators up to order } f / \mathcal{M}_{i} \text { and } 1 / \lambda & 148\end{array}$ 


\section{Purpose and motivation}

An extremely compact and elegant formulation of the dynamics of the known fundamental particles is given by the so-called Standard Model (SM) of particle physics, which has withstood all experimental tests since its conception around forty years ago. Aside from strong interactions, the Lagrangian of the SM describes both the electromagnetic and weak interactions by an $S U(2)_{L} \times U(1)_{Y}$ gauge-invariant theory. In 2012 LHC data showed the existence of a spin zero resonance of mass around $125 \mathrm{GeV}[1,2]$. This particle has been identified with the boson of the Higgs mechanism [3-6], responsible for the mass of the SM particles.

Nevertheless, despite its general success, there remain observations without explanation within the SM. Only around $5 \%$ of the Universe energy content is explained by ordinary matter. Then, around $27 \%$ of the energy balance seems to require an explanation in terms of particles, the so-called "dark matter", not accounted for in the SM, while the rest is known as the "dark energy", which encodes the accelerated expansion of the Universe. Besides, the SM does not explain massive neutrinos and it contains no mechanism to describe the matter/antimatter asymmetry present in the Universe either.

Moreover, the SM has three unsatisfactory internal issues: it provides no hint about the hierarchy in particle flavors observed in nature (also known as the flavor puzzle), the absence of $\mathrm{CP}$ violation in strong interactions requires a stringent fine-tuning and the Higgs mass seems unnaturally light if there is New Physics (NP) to which it couples. The latter is often referred to as the so-called electroweak (EW) hierarchy problem. This might not be a real problem in the sense that the SM is mathematically consistent; but taking naturalness seriously could be a way to provide a good intuition on NP. Furthermore, Higgs physics has an impact on most of the SM problems mentioned above. As a consequence, a different nature of the Higgs particle than the SM one can lead to a change in the overall current picture.

Despite having a lot of information on the interactions of the Goldstone bosons (GBs), which are absorbed to become the longitudinal components of the EW gauge bosons, little is known about the underlying dynamics of the electroweak symmetry breaking (EWSB). Learning about the true nature of the Higgs particle is thus key to clarify this and here lies the main motivation for this thesis. A simple way to evade the hierarchy problem is the introduction of a symmetry that "protects" the Higgs mass from large quantum corrections. For instance, supersymmetry or alternatively, a pseudo-Goldstone boson (pGB) nature for the Higgs particle [7] are two main approaches to the problem. Correspondingly, whether the underlying 
Higgs dynamic is weak, as in supersymmetry, or strong, as in composite Higgs models, is still in debate. Besides, while present data are compatible with the physical Higgs being embedded in an exact SM doublet representation, departures from that behavior are still viable within the present experimental accuracy of the Higgs measured couplings. For example, the Higgs could have a singlet component, as in dilaton-like models.

In our analysis we will have in mind as inspiration the possibility that the Higgs boson would descend from a GB produced in the breaking of some global symmetry at high energies. When this symmetry is explicitly broken in a soft way, an effective scalar potential is generated, in such a way that the Higgs acquires a light mass and the EWSB is triggered. Typically, in these theories the Higgs particle does not behave as an exact doublet.

In order to undertake this exploration in a model-independent way, we have first used an Effective Field Theory (EFT) approach. Among the two effective expansions commonly used in the literature to study physics Beyond the Standard Model (BSM), only the Standard Model Effective Field Theory (SMEFT), where the Higgs is an exact SM doublet at low-energies and EWSB is realized linearly, has been extensively studied at next-to-leading order (NLO) [8-15]. The alternative approach is that of Higgs Effective Field Theory (HEFT), which is built upon the chiral symmetry of the GB sector of the SM [16-18]. The GBs appear as decoupled from the physical Higgs scalar, whose couplings are encoded in generic functions [19-21]. Therefore, this effective Lagrangian allows by construction to study the possibility that the Higgs particle is not part of an exact doublet at low energies and thus it is especially appropriated to the goal of this study.

The renormalization of the chiral Lagrangian with a decoupled Higgs particle had already been discussed in the past [16,22-24], and has only been studied to some extent when including a light Higgs [25-28]. We will first explore the one-loop renormalization of the scalar sector of the HEFT basis, and further extend the analysis to the complete Lagrangian in a second step. In this way, we explicitly determine all the counterterms required by the renormalization procedure to be present at NLO, given the initial leading order (LO) Lagrangian chosen.

In order to examine the idea of a Higgs with a pGB nature, we will also develop a complete renormalizable model, inspired by the linear sigma model that describes pions as pGBs in QCD [29]. This is in contrast to most analyses in the literature [3033], performed in non-linear (effective) implementations.

The scalar sector of the model is based on the well-known minimal possibility to have EWSB with a Higgs as pGB by implementing an approximate global $S O(5)$ symmetry spontaneously broken to $S O(4)$. The renormalizable implementation contains an extra scalar, $\sigma$, singlet of the SM. We extend in this way simpler previous studies [34] and discuss its impact on phenomenology and constraints from precision observables and LHC data.

The model might be considered as a renormalizable ultraviolet (UV) completion of some deeper dynamics, or be regarded as a renormalizable model made out of elementary fields. Indeed, the mass of the extra scalar $\sigma$ will be used as a tool to 
gain intuition on the UV completion for a Higgs as pGB. Taking the limit of infinite $\sigma$ mass we have explicitly determined the corresponding benchmark effective Lagrangian, which should be a subset of the general effective Lagrangian mentioned above. In addition, with such an analysis we can also provide the first linear corrections to the non-linear effective theory.

The analysis of the scalar sector of the renormalizable model is rather general and would share similar features with any model containing a $S O(5) / S O(4)$ breaking pattern. However, the heavy fermion sector, introduced in order to induce masses for the SM fermions through "partial compositeness" [35] and be the dominant source for the explicit breaking of $S O(5)$, is quite model-dependent. With the intention of studying and classifying different alternative fermion options available in the literature, we will develop a method based on the parametrization of effective low-energy Yukawa interactions with respect to different $S O(5)$ heavy fermion embeddings.

In a similar spirit, the construction of the basis of leading operators non-invariant under baryon number within the HEFT is also presented as a complementary study, with a comparison with the SMEFT approach and a detailed discussion on the counting of the independent flavor contractions for a generic number of fermion families. The Hilbert series technique to count the number of independent structures is also considered.

In summary, this thesis is structured around the examination of the possibility of the Higgs as a pGB. To do this, both HEFT and the phenomenological study of a simple toy model have been employed. We refer the reader to the different chapters in order to understand the different analyses in more detail. 


\section{Objetivo y motivación}

El Modelo Estándar de la física de partículas constituye una formulación elegante y extremadamente compacta de las interacciones de las partículas fundamentales conocidas, el cual ha resistido todas las pruebas experimentales desde su concepción hace alrededor de cuarenta años. Además de las interacciones fuertes, el Lagrangiano del ME describe las interacciones electromagnéticas y débiles usando una teoría con simetría gauge $S U(2)_{L} \times U(1)_{Y}$. En 2012, datos del LHC mostraron la existencia de una resonancia de spin cero y masa alrededor de $125 \mathrm{GeV}[1,2]$. Esta partícula ha sido identificada con el bosón del mecanismo de Higgs [3-6], responsable de la masa de las partículas del ME.

En cualquier caso, a pesar de su éxito, hay observaciones sin explicación dentro del ME. Del contenido en energía del Universo sólo en torno al 5\% es explicado por la materia ordinaria. Alrededor del $27 \%$ parece requerir una explicación en términos de partículas, la llamada "materia oscura", la cual está fuera del ME, y el resto constituye la conocida como "energía oscura", relacionada con la expansión acelerada del Universo. Por otro lado, el ME no explica la masa de los neutrinos y tampoco contiene ningún mecanismo que describa la asimetría de materia/antimateria presente en el Universo.

Además, el ME tiene tres problemas internos sin solución satisfactoria: no proporciona ninguna pista acerca de la jerarquía en los distintos sabores de partículas observadas en la Naturaleza (también conocido como el "puzzle de sabor"). Por otro lado, la ausencia de violación de $\mathrm{CP}$ en las interacciones fuertes requiere severos ajustes y la masa del Higgs parece demasiado ligera si hubiera Nueva Física (NF) con la que se acopla. Esto último suele ser referido como el problema de la jerarquía electrodébil (ED). Esto podría no ser un verdadero problema en el sentido de que el ME es consistente matemáticamente; pero tomando los argumentos de naturalidad en serio podría ser una manera de obtener una cierta intuición de la posible NF. Adicionalmente, la física del Higgs tiene un impacto en la mayoría de problemas del ME mencionados anteriormente. Como consecuencia, una naturaleza distinta de la partícula Higgs que la del ME podría llevar a un cambio en la imagen actual.

En efecto, a pesar de tener mucha información sobre las interacciones de los bosones de Goldstone (BGs), los cuales son absorbidos para convertirse en las componentes longitudinales de los bosones gauge electrodébiles, poco se sabe acerca de las dinámicas subyacentes a la ruptura de la simetría electrodébil (RSED). Aprender acerca de la verdadera naturaleza de la partícula de Higgs es por tanto clave para clarificarlo y en ello yace la principal motivación para esta tesis. Una manera simple de evitar el 
problema de la jerarquía es introducir una simetría que "proteja" la masa del Higgs de correcciones cuánticas grandes. Por ejemplo, supersimetría o, alternativamente, un origen como pseudo-bosón de Goldstone (pBG) para la partícula de Higgs [7] son los dos principales enfoques al problema. En este sentido, si las dinámicas subyacentes al sector del Higgs son débiles, como en supersimetría, o fuertes, como en los modelos de Higgs compuesto, está todavía en debate. Aparte, aunque los datos actuales son compatibles con el Higgs físico estando embebido en una representación exacta doblete del ME, alejamientos de ese comportamiento son todavía viables dentro de la precisión experimental actual de los acoplos medidos del Higgs. Por ejemplo, el Higgs podría tener una componente que fuera singlete del ME, como en los modelos tipo dilatón.

En nuestro análisis tendremos en cuenta como inspiración la posibilidad de que el bosón de Higgs podría descender de un BG producido en la ruptura de una simetría global a altas energías. Cuando esta simetría está explícitamente rota un potencial escalar efectivo es generado, tal que el Higgs adquiere una masa ligera y se induce la RSED. Típicamente, en estas teorías la partícula de Higgs no se comporta como un doblete exacto.

Para poder realizar esta exploración de una manera independiente del modelo concreto, inicialmente hemos utilizado un enfoque de la Teoría Efectiva de Campos (TEC). De las dos expansiones efectivas usadas típicamente en la literatura para estudiar la física más allá del Modelo Estándar, sólo la Teoría Efectiva de Campos del Modelo Estándar, en la cual el Higgs es un doblete exacto del ME a bajas energías y la RSED se realiza linealmente, ha sido extensamente estudiada a segundo orden [8-15]. La alternativa es la TEC no-lineal para un Higgs ligero, la cual se basa en la simetría quiral del sector de BGs del ME [16-18]. En esta expansión los BGs aparecen desacoplados del Higgs, cuyos acoplos se encuentran codificados en funciones genéricas [19-21]. De esta manera, este Lagrangiano efectivo permite por construcción estudiar la posibilidad de que la partícula Higgs no sea parte de un doblete exacto del ME a bajas energías y por tanto es especialmente apropiada para el objetivo de este estudio.

La renormalización del Lagrangiano quiral con una partícula Higgs desacoplada ya ha sido discutida en el pasado [16,22-24], y sólo ha sido estudiado hasta cierto punto cuando un Higgs ligero es incluido [25-28]. Aquí exploraremos en primer lugar la renormalización a 1 loop del sector escalar de la base de la TEC quiral con un Higgs ligero, tras lo cual extenderemos el análisis al Lagrangiano completo en un segundo paso. De esta manera, determinaremos explícitamente todos los contratérminos requeridos a segundo orden por el procedimiento de renormalización, dado el Lagrangiano a primer orden que ha sido elegido.

Para poder explorar la idea de un Higgs con una naturaleza de pBG, estudiaremos un modelo completamente renormalizable, inspirado por el modelo sigma lineal que describe a los piones como pBGs en QCD [29]. Esto contrasta con la mayoría de análisis de la literatura [30-33], realizados en implementaciones (efectivas) nolineales.

El sector escalar del modelo está basado en la bien conocida posibilidad de tener una RSED de manera mínima con un Higgs como pBG, la cual consiste en imple- 
mentar una simetría global aproximada $S O(5)$ espontáneamente rota a $S O(4)$. La implementación renormalizable contiene un escalar extra, $\sigma$, singlete del ME. Extendemos de esta manera estudios previos más simples [34] y discutimos su impacto en la fenomenología y las restricciones provenientes de los observables de precisión y los datos del LHC.

El modelo puede considerarse que completa en el ultravioleta (UV) de manera renormalizable, codificando las dinámicas subyacentes, a los modelos no-lineales, o podría ser considerado como un modelo renormalizable que contiene campos elementales. En efecto, la masa del escalar extra $\sigma$ será usada como herramienta para ganar intuición del modelo en el UV para un Higgs como pBG. En el límite de masa del $\sigma$ infinita, determinaremos explícitamente el correspondiente Lagrangiano efectivo, el cual se espera que contenga un subconjunto de operadores pertenecientes al Lagrangiano efectivo general mencionado más arriba. Además, realizando dicho análisis también proporcionamos las primeras correcciones lineales a la teoría efectiva no-lineal.

El análisis del sector escalar del modelo es bastante general y podría compartir características similares con cualquier modelo que contenga un patrón de ruptura $S O(5) / S O(4)$. Sin embargo, el sector fermiónico pesado es bastante dependiente del modelo. Este es introducido para inducir las masas de los fermiones del ME a través de la llamada "composición parcial" [35] y ser la fuente dominante para la ruptura explícita de la simetría $S O(5)$. Con la intención de estudiar y clasificar diferentes opciones alternativas para los fermiones existentes en la literatura, desarrollaremos un método basado en la parametrización de las interacciones de Yukawa efectivas a bajas energías con respecto de las diferentes formas de incluir los fermiones en representaciones de $S O(5)$.

Como estudio complementario, se presenta también la construcción de la base de operadores no-invariantes bajo número bariónico dentro de la TEC quiral para un Higgs ligero. Se compara asimismo con la TEC del ME y se realiza una discusión detallada acerca del recuento de las contracciones de sabor independientes para un número genérico de familias de fermiones. La técnica de las series de Hilbert para contar el número de estructuras independientes también se ha tomado en cuenta.

En resumen, esta tesis se estructura alrededor del examen de la posibilidad de un Higgs como pBG. Para hacer esto, tanto técnicas de TEC como el estudio fenomenológico de un modelo simple han sido empleadas. Referimos al lector a los diferentes capítulos para entender los diferentes análisis en más detalle. 


\section{Part I}

\section{Foundations}




\section{Chapter 1}

\section{The Standard Model (SM)}

\subsection{Gauge fields and fermions}

The Standard Model (SM) of particle physics provides a compact and elegant formulation of the dynamics of the known fundamental particles. Gravity is not included, but the electromagnetic, weak and strong interactions are fully described by the theory. The model is a $S U(3)_{C} \times S U(2)_{L} \times U(1)_{Y}$ gauge invariant quantum field theory, where $S U(3)_{C}$ is the symmetry describing the strong interaction whose theory is given by quantum chromodynamics (QCD); while $S U(2)_{L} \times U(1)_{Y}$ describe the unified Electroweak (EW) force. The seminal papers that contributed to the founding of the Standard Model appeared in the sixties [36-40] and the theory has endured increasingly precise experimental tests ever since [41].

The identification of symmetries has been the source of the main achievements in the understanding of the fundamental laws in particle physics. In particular, the gauge principle is essential in this regard, since it determines the dynamics. Once the gauge symmetry is identified and implemented it leads to several testable properties regarding gauge bosons. In contrast, beyond kinetic terms, the behavior of fermions and scalars is not as constrained, producing some arbitrariness in their properties, reflected for instance in the flavor puzzle.

The importance of gauge symmetries is related to the fact that there are particles that are experimentally indistinguishable from one another under an interaction. As Yang and Mills put it in [42], the differentiation between a neutron and a proton is a purely arbitrary process, so one should be free of choosing what to call a proton or a neutron at any point in spacetime, or it would not be consistent with the idea of localized fields underlying the usual theories.

Therefore that the symmetry is gauged means that the Lagrangian is invariant under local transformations (the transformations vary depending on the point in spacetime); which implies that a bunch (their amount is the number of generators 


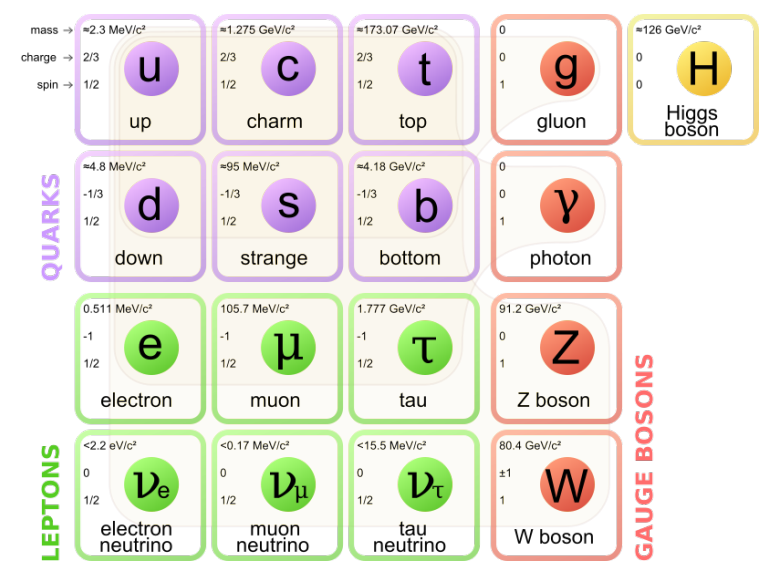

Figure 1.1: Particle content of the SM with masses and charges. On the left there are the three families of fermions. The physical gauge bosons are a combination of the ones described in this chapter. On the right, the only scalar, the Higgs boson; which will be dealt with in Chapter 3. Figure taken from https://commons . wikimedia.org/w/index . php? curid=4286964.

of the gauge group) of gauge fields have to be introduced with specific interactions with the matter content in order to preserve the symmetry. In particular, in the SM there are eight gauge fields, $G_{\mu}^{A}$ mediating the strong interaction, and four mediators of the EW interaction, $\left(W_{\mu}^{a}, B_{\mu}\right)$, three related to the $S U(2)_{L}$ group and one from the $U(1)_{Y}$ symmetry. Uppercase indices, $A \in[1,8]$, span color space, while lowercase indices, $a \in[1,3]$, span isospin space; being color $(\mathrm{C})$ and isospin $(\mathrm{T})$ the charges of the strong and weak interactions, respectively.

The kinetic terms that describe how these gauge fields propagate are elegantly written in terms of the so-called field strengths:

$$
\begin{aligned}
& \mathcal{L}_{\text {gauge }}=-\frac{1}{4} G_{\mu \nu}^{A} G^{A, \mu \nu}-\frac{1}{4} W_{\mu \nu}^{a} W^{a, \mu \nu}-\frac{1}{4} B_{\mu \nu} B^{\mu \nu}, \\
& G_{\mu \nu}^{A}=\partial_{\mu} G_{\nu}^{A}-\partial_{\nu} G_{\mu}^{A}+g_{s} f^{A B C} G_{\mu}^{B} G_{\nu}^{C}, \\
& W_{\mu \nu}^{a}=\partial_{\mu} W_{\nu}^{a}-\partial_{\nu} W_{\mu}^{a}-i g \epsilon^{a b c} W_{\mu}^{b} W_{\nu}^{c}, \\
& B_{\mu \nu}=\partial_{\mu} B_{\nu}-\partial_{\nu} B_{\mu},
\end{aligned}
$$

where $f^{A B C},-i \epsilon^{a b c}$ are the structure constants of the strong and weak group algebras, respectively, and $g_{s}$ and $g$ are the corresponding gauge couplings. These terms appear in the strong and weak cases because the symmetry groups are non-abelian. The $U(1)_{Y}$ gauge coupling will be called $g^{\prime}$ in what follows, as customary.

Besides the gauge fields, there are three families or flavors (three copies with different masses) of spin $1 / 2$ particles (fermions). As it is shown in Fig. 1.1, each generation consists of a couple of particles called leptons and a couple of particles called quarks; which makes a total of twelve particles: six quarks $(u, d, c, s, t, b)$ and 
six leptons $\left(e, \mu, \tau, \nu_{e}, \nu_{\mu}, \nu_{\tau}\right)$; which are usually displayed as flavor multiplets:

$$
\begin{aligned}
\text { up-type quarks: } & U=(u, c, t), \\
\text { down-type quarks: } & D=(d, s, b), \\
\text { charged leptons: } & E=(e, \mu, \tau), \\
\text { neutrinos: } & \nu=\left(\nu_{e}, \nu_{\mu}, \nu_{\tau}\right),
\end{aligned}
$$

The distinction between them is determined by their behavior with respect to the SM interactions. In Table 1.1 the transformation properties of the different types of fermions are summarized. For instance, only the quarks are subject to the strong interaction, transforming in the triplet representation of the $S U(3)_{C}$ group (there are indeed three copies of every quark, each with a different color); regardless of their chirality. On the other hand, both the leptons and quarks transform under the weak group; but only their left-handed (LH) components; which come in groups of two (doublets of $\left.S U(2)_{L}\right), Q_{L}$ and $L_{L}$, where the upper component has a charge under the third component of isospin, $T_{3}$, of $+1 / 2$ while the down component has $T_{3}=-1 / 2$.

\begin{tabular}{|c|c|c|c|}
\hline \hline Fermion & $S U(3)_{C}$ & $S U(2)_{L}$ & $U(1)_{Y}$ \\
\hline \hline$Q_{L}=\left(U_{L}, D_{L}\right)^{T}$ & $\mathbf{3}$ & $\mathbf{2}$ & $1 / 6$ \\
\hline$U_{R}$ & $\mathbf{3}$ & $\mathbf{1}$ & $2 / 3$ \\
\hline$D_{R}$ & $\mathbf{3}$ & $\mathbf{1}$ & $-1 / 3$ \\
\hline$L_{L}=\left(\nu_{L}, E_{L}\right)^{T}$ & $\mathbf{1}$ & $\mathbf{2}$ & $-1 / 2$ \\
\hline$E_{R}$ & $\mathbf{1}$ & $\mathbf{1}$ & -1 \\
\hline \hline
\end{tabular}

Table 1.1: Transformation properties of the fermions of the SM. 1, 2 and $\mathbf{3}$ mean they transform as the singlet (i.e. they are invariant), doublet or triplet representation of the corresponding group, respectively. The charges (hypercharges) under $U(1)_{Y}$ are shown in the last column for each particle. As originally defined, there are no right-handed neutrinos in the Standard Model.

As we were briefly introducing at the beginning of this section, preserving gauge symmetries shapes how the particles interact. This is encoded in their kinetic terms,

$$
\mathcal{L}_{k i n, f}=\bar{Q}_{L} i \not D Q_{L}+\bar{U}_{R} i \not D U_{R}+\bar{D}_{R} i \not D D_{R}+\bar{L}_{L} i \not D L_{L}+\bar{E}_{R} i \not D E_{R}
$$

since making the Lagrangian invariant under the gauge symmetries forces their derivatives to be promoted to covariant ones:

$$
\partial_{\mu} \psi \longrightarrow D_{\mu} \psi
$$




$$
\begin{gathered}
D_{\mu} Q_{L}=\left(\partial_{\mu}+i g_{s} \frac{\lambda^{A}}{2} G_{\mu}^{A}+i g \frac{\tau^{a}}{2} W_{\mu}^{a}+i g^{\prime} Q^{Y} B_{\mu}\right) Q_{L} \\
D_{\mu} Q_{R}=\left(\partial_{\mu}+i g_{s} \frac{\lambda^{A}}{2} G_{\mu}^{A}+i g^{\prime} Q^{Y} B_{\mu}\right) Q_{R} \\
D_{\mu} L_{L}=\left(\partial_{\mu}+i g \frac{\tau^{a}}{2} W_{\mu}^{a}+i g^{\prime} Q^{Y} B_{\mu}\right) L_{L} \\
D_{\mu} Q_{L}=\left(\partial_{\mu}+i g^{\prime} Q^{Y} B_{\mu}\right) E_{R}
\end{gathered}
$$

where $Q_{R}$ means any right-handed $(\mathrm{RH})$ quark; $\lambda^{A}$ and $\tau^{a}$ are the generators of the $S U(3)$ and $S U(2)$ algebras (the so-called Gell-Mann and Pauli matrices, respectively), and $Q^{Y}$ stands for the hypercharge of the fermion, which can be seen in Table 1.1. The electric charge, $\mathrm{Q}$, is related to the third component of isospin and hypercharge by a linear relation

$$
Q=T_{3}+Q^{Y}
$$

Note that both gauge bosons and fermions of the SM as described here are massless; having introduced only their kinetic terms. Possible mass terms for gauge bosons $\left(A_{\mu}\right)$ and fermions $(\psi)$

$$
A_{\mu} A^{\mu}, \quad \bar{\psi}_{L} \psi_{R}
$$

violate the SM gauge symmetry; which will prevent us from writing them in the Lagrangian.

\subsection{Renormalizability and unitarity}

Adding the Higgs sector, the SM contains 19 free parameters: the 9 fermion masses, 3 gauge couplings, the Higgs mass and vev (see Sect. 3.1), 3 mixing angles and $1 \mathrm{CP}$ phase from the Cabibbo-Kobayashi-Maskawa (CKM) matrix (the matrix determining flavor-changing charged currents due to quark mixing in the mass eigenstates), and $\theta_{Q C D}$, which characterizes the QCD vacuum*.

In order for the theory to make sense, all the free parameters have to be fixed by as many experimental observables before predictions can be made with them. Through the renormalization procedure they are used to absorb the divergences (infinities) arising in computations of physical quantities in perturbative quantum field theory. This translates in that the original parameters defining the theory in the Lagrangian will have a dependence on the energy scale after renormalization is performed, so they are said to "run" with energy; i.e. have different values in the infrared (IR) with respect to the ultraviolet (UV). This running is given by the Renormalization Group Equations (RGEs).

\footnotetext{
*If RH neutrinos with Dirac mass terms are added to the SM then there are seven additional observable parameters: the three neutrino masses plus three mixing angles and one CP-violating phase from the Pontecorvo-Maki-Nakagawa-Sakata (PMNS) matrix, the analog of the CKM matrix in the lepton sector. If neutrinos are Majorana fermions there are 2 additional physical CP phases; i.e. $19+9$ free parameters for the SM in total.
} 
Therefore, that a theory is described as renormalizable means that there is a finite set of parameters that are needed to be redefined in order to absorb all the divergences. See Chapter 6 for comments on renormalizability in effective field theories.

The Standard Model with massless gauge bosons and quarks, as presented in the previous section is renormalizable. However, weak interactions are experienced as short-range in nature, meaning that the corresponding force carriers $\left(W^{ \pm}\right.$and $Z$, as will be shown later) must be massive and therefore the EW symmetry must be somehow broken at low energies. The Higgs mechanism [4-6] is the simplest way to do this preserving gauge invariance and at the same time renormalizability in this sense (see Sect. 3.1).

There is the possibility of having massive gauge bosons in a gauge-invariant way without a Higgs particle in the spectrum; as it is described in Sect. 4.2. However, this constitutes a non-renormalizable theory. For instance, it turns out that the scattering of longitudinally polarized $W$ bosons $^{\dagger}$ grows with the square of the energy, which eventually violates the perturbative unitarity of the theory ${ }^{\ddagger}$

$$
\mathcal{A}\left(W_{L}^{+} W_{L}^{-} \rightarrow W_{L}^{+} W_{L}^{-}\right) \stackrel{E \gg M_{W}}{\simeq} \frac{g^{2}}{4 M_{W}^{2}}(s+t) \propto E^{2},
$$

where $M_{W}$ is the $W$ mass and s and t are the so-called Mandelstam variables, related to the kinematics of the process. In the case of the SM this loss of perturbativity occurs at around 2-3 TeV. There are then two possibilities: either the theory just becomes strongly coupled at high energies, thus appearing to lose unitarity in the perturbative expansion, or new degrees of freedom appear to restore unitarity, which is what indeed happens in the SM Higgs mechanism.

\footnotetext{
${ }^{\dagger}$ The gauge bosons have spin 1; so naively it seems they would possess three degrees of freedom. However, a real (i.e. not virtual) massless spin-1 boson can exist only in two transverse polarization states; while a massive spin-1 boson can also be longitudinally polarized.

$\ddagger$ The transition amplitudes of a process are the elements of the so-called S-matrix (the evolution operator describing how a physical system changes due to a scattering process) has to remain unitary in order for the theory to be consistent. This is equivalent to the statement that the sum of probabilities of all possible outcomes in the scattering process has to be 1 and this has to be preserved over time.
} 


\section{Chapter 2}

\section{Symmetry and spontaneous breaking}

\subsection{Symmetry types and breakings}

A symmetry is said to be present when there is a transformation on the variables of a system leaving the essential physics unchanged. A symmetry can either be global (the transformation is constant throughout spacetime) or gauge (the transformation is local). The concept of gauge symmetries has been very briefly described in the beginning of Sect. 1.1. The $S U(3)_{C} \times S U(2)_{L} \times U(1)_{Y}$ SM symmetry group is an example of a gauge symmetry; while $B-L$ ("baryon minus lepton number") is an example of a global symmetry of the SM Lagrangian.

Sometimes it is still useful to think in terms of symmetries in situations when the physics is invariant under a transformation just approximately. Usually in these cases the Lagrangian contains parameters such that when set to zero the symmetry is recovered. If these parameters are "small", then the predicted physical consequences that would be there if the symmetry was exact would still be approximately valid.

Depending on the dynamics of the theory, a symmetry in the Lagrangian may either remain exact or be broken in different ways:

a) The symmetry can be explicitly broken if there are terms in the Lagrangian that are not invariant under the associated transformation. These terms might be small, as in the case of an approximate symmetry. An example of this is the explicit breaking of the chiral symmetry of QCD (Quantum Chromodynamics, the modern theory describing the strong force) by quark masses. This symmetry is also broken spontaneously, see next point and Sect. 2.2.2.

b) It may happen that the ground state of the theory does not display the full symmetry of the Lagrangian and as a consequence the symmetry is lost in the spectrum of physical states; so the symmetry can be said to be hidden. Actually the symmetry is not really broken in this case; but it is realized in a 
very special way, as it will be better described in the following sections in this chapter. This may occur through scalar fields acquiring a vacuum expectation value (vev), which is the case of EWSB, the main topic of this chapter, or by quantum effects (dynamical breaking), as happens in the spontaneous breaking of QCD chiral symmetry by quark condensates.

c) Finally, it is possible that a symmetry in the Lagrangian is conserved at the classical level; but it is broken by quantum effects through an anomaly. An example of this is the case of global $U(1)$ axial symmetry of QCD.

\subsection{Spontaneous breaking of a global symmetry}

As commented above, the spontaneous breaking of a symmetry takes place in systems where the vacuum state (the lowest-energy state) is not invariant under the symmetry in the Lagrangian. A typical example is a ferromagnet where the underlying interactions are invariant under spatial rotations; while, depending on the temperature, the ground state of the system possesses two very different configurations as shown in figure 2.1 .

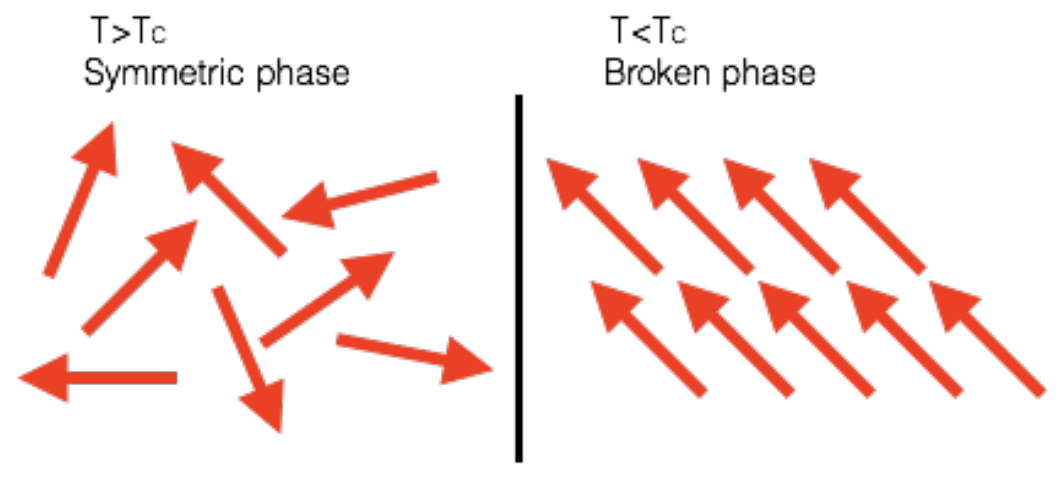

Figure 2.1: Left: Above Curie temperature, the spins describing a ferromagnetic material are randomly oriented thus making a rotational invariant ground state.

Right: Below Curie temperature, the spins are oriented in some particular direction, so the ground state is no longer invariant under rotations. The system "chooses" a particular orientation among the infinite possible ones.

The symmetric phase can be described by a paraboloid potential such as the left plot in Fig. 2.2; where there is a unique vacuum at the origin, with vanishing magnetization. On the other hand, the broken phase can be modeled by a potential with a shape that is commonly compared to that of a Mexican hat, see right plot in Fig. 2.2. There is still an extremum at the origin, which now is a local maximum; while the true vacuum is a circle of degenerate global minima. At the top of the hat everything looks symmetric in any direction; while down at the brim the symmetry seems to be lost. That is not true: the symmetry is still there; but now it is hidden.

According to Noether's theorem, there exists a classical charge $Q$; such that it is timeindependent $(d Q / d t=0)$, for each continuous transformation leaving the action 

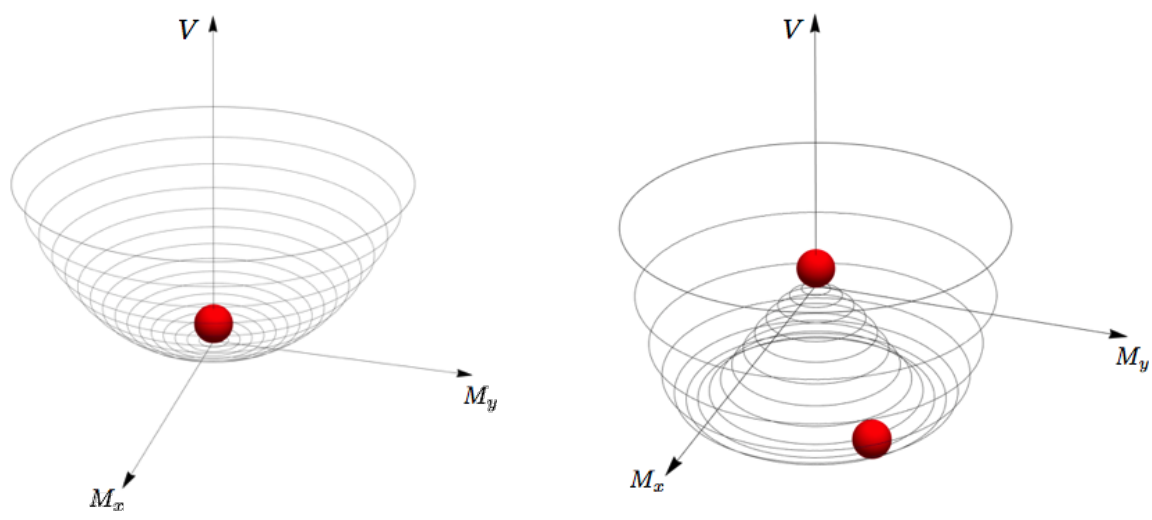

Figure 2.2: Left: Symmetric phase: the potential is a paraboloid with a unique vacuum with $|\vec{M}|=0$. A ball left to roll on this potential would simply fall and remain at this minimum.

Right: Broken phase: "Mexican hat" potential. A ball left at the origin would stay on the top of the hat; but the slightest perturbation would make it roll to the lower brim of the hat so it ends at a particular position among the infinite possible positions along the brim. That position marks the orientation of the spins after they become aligned; while the radius of the circle of minima is the modulus of the magnetization and is the same for all the possible orientations.

invariant (i.e. every symmetry of the action is related to a conservation law).

Let us consider a system with $n$ conserved charges $Q_{a}$ The associated transformation can be applied on the vacuum state, $|0\rangle$, through the unitary operators $U_{a}=e^{i \alpha_{a} Q_{a}}$

$$
|0\rangle \rightarrow e^{i \alpha_{a} Q_{a}}|0\rangle, \quad a \in[1, \ldots, n],
$$

where $\alpha_{a}$ are continuous parameters. Therefore, invariance of the vacuum means that it is annihilated by the charge operator i.e. the generator of the symmetry:

$$
U_{a}|0\rangle=e^{i \alpha_{a} Q_{a}}|0\rangle=|0\rangle \quad\left(\text { for any } \alpha_{a}\right) \quad \Rightarrow \quad Q_{a}|0\rangle=0,, \quad a \in[1, \ldots, n] .
$$

As a consequence, the vacuum is unique and the symmetry behaves in the expected way; as in the symmetric situation in Fig. 2.2.

On the other hand, in the case of a symmetry that is broken spontaneously, the vacuum is not invariant under the full symmetry. In the next example, the vacuum is invariant under $k$ generators $(k<n)$; but variant under $(n-k)$, so a subset of transformations takes the vacuum to other states,

$$
\begin{aligned}
U_{a}|0\rangle=e^{i \alpha_{a} Q_{a}}|0\rangle=|0\rangle & \Rightarrow & Q_{a}|0\rangle=0, & a \in[1, \ldots, k], \\
U_{\hat{a}}|0\rangle=e^{i \alpha_{\hat{a}} Q_{\hat{a}}}|0\rangle=\left|\alpha_{\hat{a}}\right\rangle & \Rightarrow & Q_{\hat{a}}|0\rangle \neq 0, & \hat{a} \in[k+1, \ldots, n] .
\end{aligned}
$$

Since Noether's theorem says that symmetry charges are time-independent, and thus they commute with the Hamiltonian, $d Q_{a} / d t=i\left[H, Q_{a}\right]=0$, then all states $U_{\hat{a}}|0\rangle$, which are connected by transformations of the broken symmetries, have the same energy as the vacuum, $E_{0}$,

$$
H\left|\alpha_{\hat{a}}\right\rangle=H e^{i \alpha_{\hat{a}} Q_{\hat{a}}}|0\rangle=e^{i \alpha_{\hat{a}} Q_{\hat{a}}} H|0\rangle=E_{0} e^{i \alpha_{\hat{a}} Q_{\hat{a}}}|0\rangle=E_{0}\left|\alpha_{\hat{a}}\right\rangle .
$$


Therefore, they constitute a continuous family of degenerate ground states, since the symmetry transformation is continuous.

\section{Goldstone theorem}

In simple words, the Goldstone theorem [43-45] states that

when a system has a global continuous symmetry that is not a symmetry of the vacuum, there has to exist one massless boson of spin 0 associated to each generator of the symmetry that does not preserve the ground state.

The massless particles arising due to the spontaneous breaking are referred to as Nambu-Goldstone bosons or, more simply, as we will do along this thesis, as Goldstone bosons (GBs).

Let us take a toy model with $n$ scalar fields, $\phi_{i}$, to provide a simple proof of the theorem. The Lagrangian contains the kinetic and potential terms

$$
\mathcal{L}=\frac{1}{2} \partial_{\mu} \phi_{i} \partial^{\mu} \phi_{i}-V(\phi)
$$

and is invariant under a global symmetry group whose algebra is spanned by $n$ generators, $i T_{i j}^{a}$, with $a \in[1, \ldots, n]$. Under an infinitesimal transformation, parametrized by some $\epsilon^{a} \ll 1$, the fields transform according to

$$
\phi_{i}(x) \rightarrow \phi_{i}(x)-\epsilon^{a} T_{i j}^{a} \phi_{j}(x)
$$

The invariance of the Lagrangian under the transformation imposes a condition on the potential:

$$
\begin{aligned}
\mathcal{L}\left(\phi_{i}-\epsilon^{a} T_{i j}^{a} \phi_{j}\right) & =\mathcal{L}\left(\phi_{i}\right) \\
\Rightarrow V\left(\phi_{i}-\epsilon^{a} T_{i j}^{a} \phi_{j}\right)-V\left(\phi_{i}\right) & =-\frac{\delta V}{\delta \phi_{i}} \epsilon^{a} T_{i j}^{a} \phi_{j}=0, \quad\left(\text { for any } \epsilon^{a}\right) .
\end{aligned}
$$

On the other hand, the vacuum state corresponds to a field configuration that minimizes the potential

$$
\left.\frac{\delta}{\delta \phi_{i}} V(\phi)\right|_{\left\langle\phi_{i}(x)\right\rangle}=0,
$$

with $\phi_{i}(x) \equiv\left\langle\phi_{i}(x)\right\rangle$ the fields expectation value at the vacuum (vev). If the symmetry is spontaneously broken so the vacuum is invariant only under a subset of transformations, and there are $(n-k)$ broken generators, then the analogous conditions to the ones in Eqs. (2.3) and (2.4) are:

$$
\begin{aligned}
& T_{i j}^{a}\left\langle\phi_{j}\right\rangle=0, \quad(\text { for any } i), \quad a \in[1, \ldots, k], \\
& \exists i \mid \quad T_{i j}^{\hat{a}}\left\langle\phi_{j}\right\rangle \neq 0, \quad \hat{a} \in[k+1, \ldots, n] .
\end{aligned}
$$


Differentiating Eq. (2.8), the potential invariance condition, with respect to $\phi_{k}$ and evaluating at the vacuum, gives a new condition,

$$
\frac{\delta V}{\delta \phi_{i}} T_{i j}^{a} \phi_{j}=\left.0 \Rightarrow \frac{\delta^{2} V}{\delta \phi_{i} \delta \phi_{k}}\right|_{\left\langle\phi_{i}\right\rangle} T_{i j}^{a}\left\langle\phi_{j}\right\rangle+\left.\frac{\delta V}{\delta \phi_{i}}\right|_{\left\langle\phi_{i}\right\rangle} T_{i k}^{a}=0,
$$

whose last term vanishes due to Eq. (2.9):

$$
\left.\frac{\delta^{2} V}{\delta \phi_{i} \delta \phi_{k}}\right|_{\left\langle\phi_{i}\right\rangle} T_{i j}^{a}\left\langle\phi_{j}\right\rangle=0 .
$$

According to Eq. (2.11), $T_{i j}^{\hat{a}}\left\langle\phi_{j}\right\rangle \neq 0$ for $(n-k)$ generators, so Eq. (2.13) implies that the second derivative of the potential at the vacuum has precisely $(n-k)$ eigenstates with vanishing eigenvalue. From the variation of the action, it can be seen that the excitations around the vacuum, $\pi_{i}(x) \equiv \phi_{i}(x)-\left\langle\phi_{i}(x)\right\rangle$, must satisfy the Klein Gordon equation

$$
\left(\delta_{i j} \partial_{\mu} \partial^{\mu}+\left.\frac{\delta^{2} V}{\delta \phi_{i} \delta \phi_{j}}\right|_{\left\langle\phi_{i}\right\rangle}\right) \pi_{j}(x)=0 .
$$

Therefore, the $(n-k)$ zero eigenvalues of the second derivative of the potential should correspond to $(n-k)$ massless particles in the spectrum: the Goldstone bosons.

Finally, the fact that the Lagrangian is nevertheless invariant under the $(n-k)$ transformations, means that it has to be invariant under shifts of the corresponding Goldstone fields. Therefore, when the Lagrangian is written in terms of the physical fields, it can only contain derivative couplings of the GBs. For this reason, it can be said that the shift symmetry forbids a mass term for them.

\section{Spontaneous breaking of the chiral symmetry in QCD}

The QCD Lagrangian with $n$ flavors of massless quarks has a Lagrangian*

$$
\mathcal{L}_{Q C D}=-\frac{1}{4} G_{\mu \nu}^{A} G^{A, \mu \nu}+\bar{Q}_{L} i \not D Q_{L}+\bar{Q}_{R} i \not D Q_{R}
$$

which contains only gluon and quark fields and their kinetic terms. Therefore, in this limit, as there are no mass terms mixing the left- and right- handed quark fields, the Lagrangian is invariant under a global chiral $S U(n)_{L} \times S U(n)_{R}$ symmetry. Under the associated transformations, the quark fields of each chirality transform as doublets:

$$
\begin{aligned}
Q_{L}=\left(\begin{array}{c}
U_{L} \\
D_{L}
\end{array}\right) \rightarrow L Q_{L}, & L \in S U(2)_{L}, \\
Q_{R}=\left(\begin{array}{c}
U_{R} \\
D_{R}
\end{array}\right) \rightarrow R Q_{R}, & R \in S U(2)_{R},
\end{aligned}
$$

${ }^{*}$ The so-called $\theta$-term, related to the "strong CP problem", is omitted here, as it is irrelevant for the discussion. 
where $L, R=e^{-i \alpha_{L, R}^{a} \tau^{a} / 2}$ are arbitrary $S U(2)$ matrices. However, it is expected that the quark-gluon confining gauge interaction produces quark-antiquark (spinless) condensates, whose vev is non-vanishing, at a characteristic energy scale, $\Lambda_{Q C D}$ :

$$
\langle\bar{Q} Q\rangle=\left\langle\bar{Q}_{L} Q_{R}+\bar{Q}_{R} Q_{L}\right\rangle \neq 0
$$

This vacuum configuration is not symmetric under the complete chiral symmetry; but only under the transformations with $\boldsymbol{\alpha}_{L}=\boldsymbol{\alpha}_{R}$. Therefore, the condensates are breaking spontaneously the chiral symmetry down to the vector (also called isospin or diagonal) subgroup:

$$
S U(n)_{L} \times S U(n)_{R} \rightarrow S U(n)_{V}, \quad S U(n)_{V} \equiv S U(n)_{L+R}
$$

Therefore, as the Goldstone theorem dictates, $\left(n^{2}-1\right)$ massless spinless fields should appear in the spectrum. These correspond to the $\left(n^{2}-1\right)$ generators of the broken axial symmetry $\left(\boldsymbol{\alpha}_{L}=-\boldsymbol{\alpha}_{R}\right)$; so they will behave as pseudoscalar fields (i.e. odd under a parity transformation).

It is worth noting that this is an example that the Goldstone bosons are not necessarily elemental; but they can be composite as well. In this case, they correspond to bound states of some elementary fields, the quarks. This is usually referred to as dynamical symmetry breaking.

In reality the quarks are not massless. However, the up, down and strange quarks have masses that are very small as compared to $\Lambda_{Q C D} \sim 200-300 \mathrm{MeV}$. Therefore, the Lagrangian can be considered to be approximately chiral symmetric, and thus the spontaneous breaking produces eight $(n=3$ approximately massless flavors) pseudo-Goldstone bosons, which are identified with the lighter mesons in the spectrum (pions, kaons and eta). Indeed, this way has proved to be successful in understanding the hierarchy between mesons and hadrons in QCD.

\section{Linear sigma model}

The linear sigma model [29] was introduced to study the spontaneous breaking of QCD chiral symmetry and it constitutes a very simple way to exemplify the main characteristics of a global symmetry that is spontaneously broken, which will reappear in a similar fashion when discussing the spontaneous breaking of a gauge symmetry. For simplicity, let us first just focus on the scalar part of the model and consider just a $U(1)$ global symmetry; so the Lagrangian can be simply written as that of a complex scalar field:

$$
\mathcal{L}=\partial_{\mu} \phi^{*} \partial^{\mu} \phi-V\left(\phi^{*} \phi\right)=\partial_{\mu} \phi^{*} \partial^{\mu} \phi-m^{2} \phi^{*} \phi-\lambda\left(\phi^{*} \phi\right)^{2}
$$

which is invariant under transformations of the type $\phi(x) \rightarrow e^{i \alpha} \phi(x)$, with constant $\alpha$. It turns out the potential has a very different behavior depending on the sign of $m^{2}$, as it is shown in the following figures (the self-coupling $\lambda$ is assumed positive so the potential is bounded from below): 


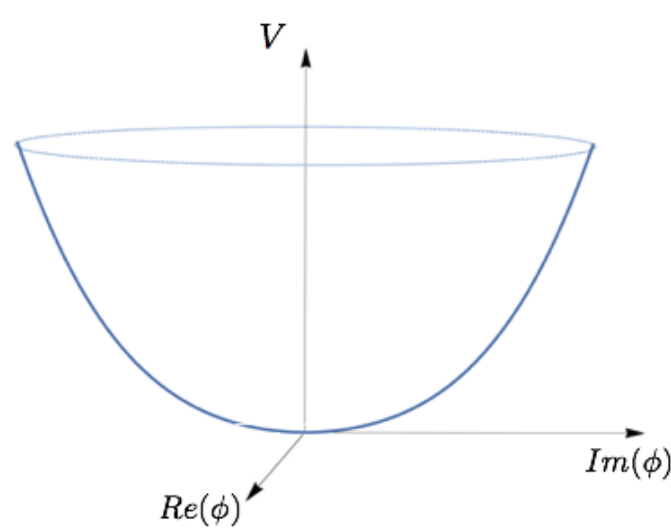

For $m^{2}>0$ the potential has a unique minimum at $\langle\phi\rangle=0$. The vacuum is symmetric under the global $U(1)$.

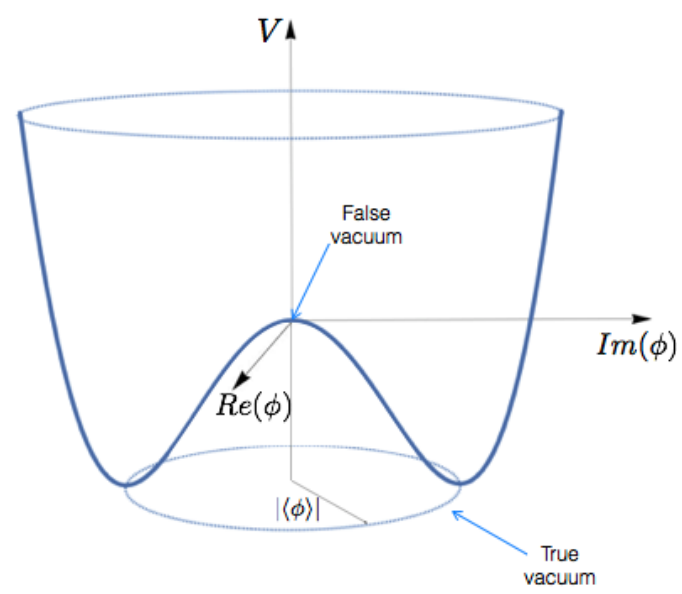

For $m^{2}<0$ the potential has a degenerate infinite set of minima, with modulus $|\langle\phi\rangle|=\sqrt{\frac{-m^{2}}{2 \lambda}} \equiv \frac{f}{\sqrt{2}}$, The vev is not invariant under $U(1)$ once a phase is chosen.

This is very similar to the two-phase behavior that was qualitatively described in Fig. 2.2. In fact, the Ginzburg-Landau theory [46], developed to study superconductivity implements a potential analogous to the scalar potential described here. In the vacuum the modulus of the field configuration is fixed; while the complex phase is arbitrary. It is the choice of a particular phase that produces the breaking (since the vev is no longer invariant under $U(1)$ ). Therefore, following Goldstone theorem, one GB field should appear in the spectrum in this case.

To analyze perturbations around the true vacuum, it is convenient to shift the field as

$$
\phi(x)=\frac{1}{\sqrt{2}}(\sigma(x)+f) e^{i \pi(x) / f_{\pi}}, \quad f^{2}=\frac{-m^{2}}{\lambda},
$$

where $\sigma$ and $\pi$ are real scalar fields and they are normalized by the appropriate constants in order to have canonical kinetic energy in the Lagrangian (see below). The GB field, $\pi(x)$, is the excitation along the circle of minima; while $\sigma(x)$ represents excitations in the perpendicular direction. The GB field should possess a shift symmetry since moving along the circle of minima does not have any potential energy cost (all minima are degenerate) and thus it is natural to write it as a phase. In other words, the symmetry is still realized as $\pi(x) \rightarrow \pi(x)+f \theta$, with $\sigma$ invariant.

The Lagrangian in terms of the shifted fields takes the form

$$
\mathcal{L}=\frac{1}{2}\left(\partial_{\mu} \sigma\right)^{2}+\frac{1}{2}\left(1+\frac{\sigma}{f}\right)^{2}\left(\partial_{\mu} \pi\right)^{2}-\frac{1}{2} m_{\sigma}^{2} \sigma^{2}-\lambda f \sigma^{3}-\frac{\lambda}{4} \sigma^{4}
$$

where it can be seen that the radial field has acquired a mass $m_{\sigma}=-2 m^{2}$ (in the broken phase $m^{2}<0$ ); while the GB field shows no mass term at all. The constant term appearing in the potential after the shift is omitted. 
This can be generalized to the spontaneous breaking of $S U(2)_{L} \times S U(2)_{R}$; which would simply translate into three GBs, $\vec{\pi}(x)=\left(\pi_{1}(x), \pi_{2}(x), \pi_{3}(x)\right)$, transforming as a triplet under the $S U(2)$ isospin group, and a $\sigma$ field, singlet under $S U(2)$, which will acquire a non-zero vev. Actually, there is an isomorphism between the Lie algebras of $S U(2)_{L} \times S U(2)_{R}$ and $S O(4)$, so it is convenient to write an $S O(4)$ invariant Lagrangian in terms of the matrix field

$$
\Sigma=\sigma \mathbb{1}+i \vec{\tau} \cdot \vec{\pi}
$$

transforming as a bidoublet of $S U(2)_{L} \times S U(2)_{R}$ :

$$
\Sigma(x) \rightarrow L \Sigma(x) R^{\dagger}
$$

so the potential and its minimum now are

$$
V\left(\Sigma^{\dagger} \Sigma\right)=\frac{m^{2}}{4} \operatorname{Tr}\left(\Sigma^{\dagger} \Sigma\right)+\frac{\lambda}{16}\left(\operatorname{Tr}\left(\Sigma^{\dagger} \Sigma\right)\right)^{2}, \quad \frac{\left\langle\operatorname{Tr}\left(\Sigma^{\dagger} \Sigma\right)\right\rangle}{2}=\frac{-m^{2}}{\lambda} \equiv f^{2} .
$$

where $\frac{1}{2} \operatorname{Tr}\left(\Sigma^{\dagger} \Sigma\right)=\sigma^{2}+\vec{\pi} \cdot \vec{\pi}$. Using the expression for the minimum of the potential and choosing it to be aligned with the $\sigma$ field, so

$$
\langle\Sigma\rangle=f \mathbb{1}, \quad \leftrightarrow \quad\langle\sigma\rangle=f, \quad\langle\vec{\pi}\rangle=0,
$$

so the $\pi_{i}$ fields are the GBs. It is actually clearer to add a constant term and recast the potential in the form

$$
V=\frac{\lambda}{4}\left(\sigma^{2}+\vec{\pi} \vec{\pi}-f^{2}\right)^{2}
$$

where $f^{2}=-m^{2} / \lambda$. Shifting the fields by the chosen vevs it can be seen the $\sigma$ particle acquires the following mass, while the $\pi_{i}$ fields remain massless:

$$
m_{\sigma}^{2}=2 \lambda f^{2}=-2 m^{2}, \quad m_{\pi_{i}}=0 .
$$

\section{Non-linear sigma model}

The previous discussion is an example of a spontaneous breaking of a symmetry that is realized in a linear way. It is however possible to implement the spontaneous breaking in a non-linear fashion by integrating out the radial field (so only the GBs remain in the spectrum).

The non-linear sigma model can be generated by taking $m_{\sigma} \rightarrow \infty$ keeping $f$ constant (or, equivalently, taking $\lambda \rightarrow \infty$ keeping $f$ constant), which leads to the non-linear constraint

$$
\Sigma^{\dagger} \Sigma=f^{2} \mathbb{1} .
$$

The parametrization of the $\Sigma$ matrix field in terms of the GBs is indeed not unique, as long as this condition is satisfied (see Sect. 6.2 for further details on this). In the parametrization described in the previous section, $\Sigma=\sigma \mathbb{1}+i \vec{\tau} \vec{\pi}$, the non-linear condition enforces that $\sigma(x)=\left(f^{2}-\vec{\pi}^{2}(x)\right)^{1 / 2}$, so now the $\pi_{i}(x)$ fields transform non-linearly under $S U(2)_{L} \times S U(2)_{R}$ due to the missing bidoublet component that 
has been integrated out. In the decoupling limit, for this parametrization, the Lagrangian reduces to

$$
\mathcal{L}_{N L}=\frac{f^{2}}{4} \operatorname{Tr}\left(\partial_{\mu} \mathbf{U}^{\dagger} \partial^{\mu} \mathbf{U}\right)
$$

Taking the self-coupling to infinity obviously spoils perturbation theory. However, this is just a consequence of the non-linear theory being perturbatively nonrenormalizable (the renormalization of the effective expansion will be discussed in Sect. 6.1). In fact, the linear theory can be thought of as the UV completion of the non-linear model; with a finite $m_{\sigma}$ as a cut-off to regulate the theory.

Alternatively, the polar parametrization,

$$
\Sigma(x)=\varphi(x) \mathbf{U}(x), \quad \mathbf{U}(x)=e^{i \frac{\vec{\tau} \vec{\pi}^{\prime}(x)}{f}},
$$

can be used. It is analogous to the one used in Eq. (2.20). The non-linear condition now simply reduces to $\varphi(x)=f$ and the corresponding non-linear Lagrangian is found to be

$$
\mathcal{L}_{N L}=\frac{1}{2} \partial_{\mu} \vec{\pi} \cdot \partial^{\mu} \vec{\pi}+\frac{1}{2} \frac{\left(\vec{\pi} \cdot \partial_{\mu} \vec{\pi}\right)^{2}}{f^{2}-\vec{\pi}^{2}} .
$$

The fields $\varphi$ and $\hat{\pi}$ are related to the previous $\sigma$ and $\vec{\pi}$ used in $\Sigma=\sigma \mathbb{1}+i \vec{\tau} \vec{\pi}$ through a non-linear change of variables:

$$
\sigma=\varphi \cos \left(\frac{\left|\overrightarrow{\pi^{\prime}}\right|}{f}\right), \quad \pi_{i}=\varphi \frac{\pi_{i}^{\prime}}{f} \sin \left(\frac{\left|\overrightarrow{\pi^{\prime}}\right|}{f}\right)
$$

In this case all the GBs degrees of freedom are contained in the matrix $\mathbf{U}$, in a way which retains the chiral behavior ( $\mathbf{U}$ transforms as $\mathbf{U} \rightarrow L \mathbf{U} R^{\dagger}$ ). This is the reason why this parametrization is useful in constructing an effective theory in which the EWSB is realized non-linearly, in which case there is no need of a Higgs field (at the expense of losing renormalizability).

As discussed above, the Lagrangian is invariant under the shifts of the GB fields, associated to the broken symmetries of the vacuum, which in this case corresponds to the axial symmetries. The corresponding Noether currents have the expression, at first-order:

$$
j_{A}^{\mu a}=-f \partial^{\mu} \pi^{a}
$$

Therefore, defining $|\pi\rangle$ as the state created and annihilated from the vacuum, the amplitude for the creation of a pion $\pi$ from the vacuum with momentum $p$,

$$
\left\langle 0\left|j_{A}^{\mu a}\right| \pi^{b}\right\rangle=i f p^{\mu} \delta^{a b},
$$

gives the physical interpretation of $f$ as the pion decay constant. This can be measured, for instance, from the decay $\pi^{+} \rightarrow \mu^{+} \nu_{\mu}$, yielding $f \approx 92 \mathrm{MeV}$. 


\subsection{Spontaneous breaking of a gauge symmetry}

The mechanism by which spontaneous symmetry breaking occurs when the symmetry is local is also called the Higgs mechanism. Historically, this was developed after the efforts of different people [3-6] and drew heavily from previous work in Solid State Physics [47], which, in turn, was motivated by previous work by Schwinger $[48,49]$. See also earlier work by Stueckelberg [50].

When the global symmetry in (2.7) is made local, the field transforms as

$$
\phi_{i}(x) \rightarrow \phi_{i}(x)-\epsilon^{a}(x) T_{i j}^{a} \phi_{j}(x) .
$$

As it was introduced in 1.1, a set of gauge bosons, $A_{\mu}^{a}(x)$, has to be introduced in order to respect the gauge symmetry transforming as

$$
A_{\mu}^{a}(x) \rightarrow A_{\mu}^{a}(x)-\frac{1}{g} \partial_{\mu} \epsilon^{a},
$$

so they will make $D_{\mu} \phi$ transform in the same way as $\phi$ under the gauge symmetry, with the covariant derivative is defined as

$$
D_{\mu} \phi_{i}(x)=\partial_{\mu} \phi_{i}(x)-g A_{\mu}^{a}(x) T_{i j}^{a} \phi_{j}(x)
$$

where $g$ is the gauge coupling constant. Using these definitions the Lagrangian for $n$ scalar fields of Eq. (2.6) becomes

$$
\mathcal{L}=-\frac{1}{4} A_{\mu \nu}^{a} A^{a \mu \nu}+\frac{1}{2} D_{\mu} \phi_{i} D^{\mu} \phi_{i}-V(\phi),
$$

Expanding the scalar kinetic term yields

$$
\frac{1}{2} D_{\mu} \phi_{i} D^{\mu} \phi_{i}=\frac{1}{2} \partial_{\mu} \phi_{i} \partial^{\mu} \phi_{i}-g A^{a \mu} \partial_{\mu} \phi_{i}\left(T^{a} \phi\right)_{i}+\frac{1}{2} g^{2} A_{\mu}^{a} A^{b \mu}\left(T^{a} \phi\right)_{i}\left(T^{b} \phi\right)_{i}
$$

where the last piece has the structure of a mass term for the gauge bosons:

$$
\frac{1}{2} m_{a b}^{2} A_{\mu}^{a} A^{b \nu}
$$

Let us assume some of the scalars get a vev such that they make the vacuum noninvariant under $(n-k)$ of the symmetry generators, $T^{\hat{a}}$, so we expand them around the vacuum

$$
\phi_{i}(x)=\langle\phi\rangle_{i}+\varphi_{i}(x) .
$$

Then the gauge boson mass matrix is

$$
m_{a b}^{2}=g^{2}\left(T^{a}\langle\phi\rangle\right)_{i}\left(T^{b}\langle\phi\rangle\right)_{i}=m_{a i} m_{b i},
$$

where $m_{a i} \equiv g\left(T^{a}\langle\phi\rangle\right)_{i}$. When one of the generators leave the vacuum invariant $\left(T^{a}\langle\phi\rangle=0\right)$, its contribution to the mass matrix vanishes and thus the associated gauge boson will remain massless. 
At the vacuum, from the second term in Eq. (2.39), the Lagrangian will also contain terms of the form

$$
-g A_{\mu}^{\hat{a}} \partial^{\mu} \varphi_{k}\left(T^{\hat{a}}\langle\phi\rangle\right)_{k}
$$

which are non-zero only for the broken generators $\left(T^{\hat{a}}\langle\phi\rangle \neq 0\right)$. As shown in the discussion in Sect. 2.2, the broken generators connect all the degenerate states at the minimum of the potential; so the states $\partial^{\mu} \varphi_{k}$ appearing in this term, being parallel to $\left(T^{\hat{a}}\langle\phi\rangle\right)_{k}$, have to be the excitations along these directions, and thus, the field fluctuations around the set of minima; i.e. the Goldstone bosons. Let us change their name to $\chi_{i}(x)$ in order to distinguish them from the bosons with non-zero vev, $\varphi(x)$, so the term above can be written as

$$
-g A_{\mu}^{\hat{a}} \partial^{\mu} \chi_{k} T_{k j}^{\hat{a}}\langle\phi\rangle_{j}=-m_{\hat{a} k} A_{\mu}^{\hat{a}} \partial^{\mu} \chi_{k} .
$$

In order to understand how the physical gauge bosons will become massive, it is instructive to compute their two-point function. Let us define $i \Pi_{\mu \nu}\left(q^{2}\right)$ as the sum of all one-particle-irreducible $(1 \mathrm{PI})^{\dagger}$ insertions in the propagator. The Ward identity, which is a consequence of gauge invariance, requires that it is completely transverse:

$$
i \Pi_{\mu \nu}^{a b}\left(q^{2}\right)=i \delta_{a b} q^{2}\left(g_{\mu \nu}-\frac{q_{\mu} q_{\nu}}{q^{2}}\right) \Pi\left(q^{2}\right),
$$

where $\Pi\left(q^{2}\right)$ is the vacuum polarization amplitude. The two terms of Eqs. (2.42) and (2.44) can be treated as vertices in perturbation theory, so at leading order they contribute to the gauge bosons two-point function. Thus, at leading order,

$$
\begin{aligned}
& \overbrace{}^{a \mu} \sim \sim \overbrace{}^{b \nu}+\overbrace{-i m_{a i} q_{\mu}}^{a \mu} \underset{-i m_{i} q_{\nu}}{\chi x_{j}} \sim^{b \nu} \\
& =i \Pi_{\mu \nu}^{a b}\left(q^{2}\right)=i m_{a b}^{2} g_{\mu \nu}-m_{a i} q_{\mu} \frac{i \delta_{i j}}{q^{2}} m_{b j} q_{\nu}=i m_{a b}^{2}\left(g_{\mu \nu}-\frac{q_{\mu} q_{\nu}}{q^{2}}\right),
\end{aligned}
$$

where it can be seen that the GBs have provided the necessary contribution to make the polarization amplitude transverse. Therefore, by comparison with Eq. (2.45), it is found

$$
\Pi\left(q^{2}\right)=\frac{m_{a b}^{2}}{q^{2}},
$$

In terms of the exact two-point function, which is an infinite geometric series of all the 1PI insertions in the propagator (which is $-i g_{\mu \nu} / q^{2}$ before the spontaneous breaking takes place), it can be resummed as

$$
\frac{-i}{q^{2}} \frac{1}{1-\Pi\left(q^{2}\right)}\left(q^{2} g_{\mu \nu}-\frac{q_{\mu} q_{\nu}}{q^{2}}\right),
$$

the gauge bosons have become massive because the GBs have created the necessary pole in $q^{2}=m^{2}$ :

$$
\frac{-i}{q^{2}-m^{2}}\left(q^{2} g_{\mu \nu}-\frac{q_{\mu} q_{\nu}}{q^{2}}\right)
$$

\footnotetext{
${ }^{\dagger} 1 \mathrm{PI}$ diagrams are such that cannot be separated in two pieces when one internal line is cut.
} 
The kinetic mixing of Eq. (2.44) is quite inconvenient because it mixes the propagators of the gauge and Goldstone bosons. This is indeed a reflection from the fact that Goldstone bosons are not actual physical massless particles; since the mixing term should not appear in the Lagrangian once the fields have been rotated to the physical basis. We can use the freedom of Eq. (2.36) to redefine the gauge boson field in such a way that the Goldstone bosons disappear from the theory ${ }^{\ddagger}$.

However, it is easier to see it at work in a simple example. For instance, in the spontaneous breaking of a gauge $U(1)$ symmetry, with a complex field $\phi \rightarrow e^{i \alpha(x)} \phi$, with Lagrangian

$$
\mathcal{L}=\frac{1}{4} F_{\mu \nu} F^{\mu \nu}+D_{\mu} \phi^{\dagger} D^{\mu} \phi-V\left(\phi^{\dagger} \phi\right)
$$

where

$$
D_{\mu} \phi_{i}=\partial_{\mu} \phi_{i}-i e A_{\mu} \phi, \quad A_{\mu} \rightarrow A_{\mu}^{\prime}-\frac{1}{e} \partial_{\mu} \alpha
$$

and the field is parametrized as

$$
\phi=\frac{\sigma}{\sqrt{2}} U(\pi), \quad U(\pi) \equiv e^{i \pi / f}
$$

where $\sigma$ is the field acquiring a non-zero vev $(f)$ and $\pi$ is the Goldstone boson field. Therefore, the kinetic terms from $D_{\mu} \phi^{\dagger} D^{\mu} \phi$, involving the GB and the gauge boson, once the $\sigma$ field has acquired a vev $(\sigma \rightarrow \sigma+f)$, are

$$
\frac{1}{2}\left(\partial_{\mu} \pi\right)^{2}+\frac{1}{2} e^{2} f^{2} A_{\mu}^{2}-e f A_{\mu} \partial^{\mu} \pi=\frac{1}{2} e^{2} f^{2}\left(A_{\mu}-\frac{1}{e f} \partial_{\mu} \pi\right)^{2} .
$$

Therefore, the specific gauge choice to make the GB disappear in the Lagrangian (the unitary gauge) is simply $\alpha(x)=-\partial_{\mu} \pi(x) / f$, so the gauge field is redefined as

$$
A_{\mu}^{\prime}=A_{\mu}-\frac{1}{e f} \partial_{\mu} \pi
$$

and the expression above is reduced to $\frac{1}{2} e^{2} f^{2} A_{\mu}^{\prime 2}$ and thus the gauge field has acquired a mass

$$
m_{A}=e f
$$

Besides, the scalar field has also to change accordingly due to the gauge transformation, so

$$
\phi \rightarrow e^{i \alpha} \phi=U(\pi)^{-1} \frac{\sigma+f}{\sqrt{2}} U(\pi)=\frac{\sigma+f}{\sqrt{2}}
$$

and it is clear the GB has disappeared in this gauge.

\footnotetext{
${ }^{\ddagger}$ It is also possible to do this by introducing explicitly the gauge-fixing term required by the Faddeev-Popov quantization procedure, which in the $R_{\xi}$ gauges can be chosen of the form

$$
\mathcal{L}_{G F}=\frac{1}{2 \xi}\left(\partial_{\mu} A^{a \mu}-\xi g T_{k i}^{a}\langle\phi\rangle_{i} \chi_{k}\right)^{2} .
$$

In this case, the kinetic mixing term between the gauge bosons and the GBs is cancelled automatically. The Goldstone bosons acquire a mass proportional to the gauge fixing parameter, $\xi$, (a gauge-dependent mass is another sign that they are in reality unphysical particles), so the unitary gauge corresponds to the $\xi \rightarrow \infty$ limit, where they can be integrated out.
} 


\section{Chapter 3}

\section{The Higgs of the SM}

\subsection{The EWSB mechanism}

In the SM we want to generate masses for three gauge fields: $W^{+}, W^{-}$and $Z$; while keeping the photon, $A$, massless (since the symmetry breaking only affects the EW gauge sector, the gluons will remain massless all along). That makes three degrees of freedom will be needed to become the longitudinal modes of the massive bosons. As explained throughout the previous chapter, one needs to introduce a new field with a potential analogous to the one in Eq. (2.19) that keeps the Lagrangian invariant under $S U(2)_{L} \times U(1)_{Y}$; while making the vacuum not invariant under the $\mathrm{EW}$ gauge symmetry. Therefore, the new degrees of freedom must be charged under both the weak and hypercharge symmetries in order to break the complete gauge group. The simplest solution to this that also preserves the renormalizability of the Lagrangian is to introduce a complex $S U_{L}(2)$ doublet, $\Phi$, with hypercharge $Q^{Y}=1 / 2$, the so-called Higgs doublet:

$$
\Phi=\left(\begin{array}{c}
\Phi^{+} \\
\Phi^{0}
\end{array}\right)
$$

The charges are chosen according to Eq. (1.4) in a way that the electromagnetic subgroup, $U(1)_{e m}$, is preserved, so the component acquiring a non-zero vev in the symmetry breaking must be electrically neutral.

The most general gauge invariant Lagrangian for the Higgs field that can be constructed with renormalizable interactions $(d \leq 4)$ is:

$$
\mathcal{L}_{\Phi}=D_{\mu} \Phi^{\dagger} D^{\mu} \Phi-V(\Phi)-\left[\bar{Q}_{L} \tilde{\Phi} Y_{U} U_{R}+\bar{Q}_{L} \Phi Y_{D} D_{R}+\bar{L}_{L} \Phi Y_{E} E_{R}+\text { h.c. }\right],
$$

where

$$
D_{\mu} \Phi=\partial_{\mu} \Phi+\frac{i g}{2} W_{\mu}^{a} \tau^{a} \Phi+\frac{i g^{\prime}}{2} B_{\mu} \Phi
$$

and $Y_{U}, Y_{D}, Y_{E}$ are the so-called Yukawa matrices, which contain the couplings of the Higgs boson with the three families of fermions. The Yukawa term for the up-type 
quark has to be constructed with the charge conjugate of the Higgs field in order to preserve the EW gauge group:

$$
\tilde{\Phi}=i \tau^{2} \Phi^{*}=\left(\begin{array}{c}
\Phi^{0 *} \\
-\Phi^{-}
\end{array}\right)
$$

where the complex conjugation makes the term invariant under hypercharge and the antisymmetric tensor, $\epsilon_{i j}=\left(i \tau^{2}\right)_{i j}$, contracts the left-handed doublets in a way $S U(2)_{L}$ is preserved.

According to Sect. 2.3 the scalar potential should have a form

$$
V(\Phi)=\mu^{2} \Phi^{\dagger} \Phi+\frac{\lambda}{2}\left(\Phi^{\dagger} \Phi\right)^{2}, \quad \lambda>0
$$

In the non-symmetric phase $\left(\mu^{2}<0\right)$ it can be minimized with

$$
\langle\Phi\rangle=\sqrt{\frac{-\mu^{2}}{\lambda}}\left(\begin{array}{l}
0 \\
1
\end{array}\right) \equiv \frac{v}{\sqrt{2}} .
$$

Excitations around this vacuum can be parametrized in polar coordinates as

$$
\Phi(x)=\frac{v+h(x)}{\sqrt{2}} \mathbf{U}(x)\left(\begin{array}{l}
0 \\
1
\end{array}\right), \quad \mathbf{U}(x)=e^{i \pi^{a}(x) \tau^{a} / v},
$$

where it is made explicit that $\Phi$ contains four real degrees of freedom: three phases (the would-be-GBs), $\pi_{i}(x)$, and a radial excitation, $h(x)$, the physical Higgs boson field that remains in the particle spectrum. The $\mathbf{U}$ can be expanded as

$$
\Phi(x)=\frac{1}{\sqrt{2}}\left(\begin{array}{c}
i \pi_{1}(x)+\pi_{2}(x) \\
v+h(x)-i \pi_{3}(x)
\end{array}\right)+\mathcal{O}\left(\pi_{i}^{2}\right) .
$$

It is possible to eliminate the unphysical would-be-GBs fields $\vec{\pi}(x)$ doing a gauge transformation (i.e. going to the unitary gauge), so they are "eaten" by the gauge boson fields (see Sect. 2.3).

Expanding Eq. (3.3) one finds

$$
D^{\mu} \Phi=\partial_{\mu} \Phi+\frac{i v}{\sqrt{8}}\left(\begin{array}{c}
g\left(W_{1}-i W_{2}\right) \\
\left(-g W_{3}+g^{\prime} B_{\mu}\right)
\end{array}\right) .
$$

Therefore, in terms of the physical gauge fields defined as

$$
\begin{aligned}
& W_{\mu}^{ \pm}=\frac{1}{\sqrt{2}}\left(W_{\mu}^{1} \mp i W_{\mu}^{2}\right), \\
& Z_{\mu}=\frac{1}{\sqrt{g^{2}+g^{\prime 2}}}\left(g W_{\mu}^{3}-g^{\prime} B_{\mu}\right), \\
& A_{\mu}=\frac{1}{\sqrt{g^{2}+g^{\prime 2}}}\left(g^{\prime} W_{\mu}^{3}+g B_{\mu}\right),
\end{aligned}
$$

the kinetic term for the Higgs field in the unitary gauge has the form

$$
\left(D_{\mu} \Phi\right)^{\dagger}\left(D^{\mu} \Phi\right)=\frac{1}{2} \partial_{\mu} h \partial^{\mu} h+\frac{(v+h)^{2}}{4} g^{2} W_{\mu}^{+} W^{-\mu}+\frac{(v+h)^{2}}{8}\left(g^{2}+g^{\prime 2}\right) Z_{\mu} Z^{\mu}
$$


From this equation, the masses for the $W$ and $Z$ bosons can be read:

$$
m_{W}=\frac{g v}{2}, \quad m_{Z}=\frac{\sqrt{g^{2}+g^{\prime 2}} v}{2},
$$

while the photon, $A_{\mu}$, remains massless.

Eq. (3.1) shows how the weak gauge bosons $W^{1}$ and $W^{2}$ combine in the physical $W^{ \pm}$bosons; while $W^{3}$ and $B_{\mu}$ combine to yield the $Z$ boson and the photon. The angle that rotates the mass matrix to provide the neutral mass eigenstates is the weak angle, $\theta_{W}$, which is related to the gauge coupling constants as

$$
\theta_{W}=\arctan \left(\frac{g^{\prime}}{g}\right)
$$

which is not a prediction of the SM; but a free parameter, with $\sin ^{2} \theta_{W} \simeq 0.23$ from experimental data [41]. The electromagnetic coupling is

$$
e=g \sin \theta_{W}=g^{\prime} \cos \theta_{W}
$$

Going back to the scalar sector, the potential for $h$ is

$$
V(h)=\frac{m_{h}^{2}}{2} h^{2}+\frac{\lambda v}{2} h^{3}+\frac{\lambda}{8} h^{4}, \quad m_{h}^{2}=-2 \mu^{2}=\lambda v^{2} .
$$

Notice that both $m_{h}$ and $\lambda$ are unknown parameters in the SM model. However, it turns out that the vacuum expectation value of the Higgs field, $v$ is precisely the EW energy scale, $v_{E W}$, which was known from experiments long before the Higgs mechanism was proposed. They can be related through the Fermi constant, $G_{F}$ and Eq. (3.12):

$$
\frac{G_{F}}{\sqrt{2}}=\frac{1}{2 v_{E W}^{2}}=\frac{g^{2}}{8 m_{W}^{2}} \Rightarrow v=v_{E W}=246 \mathrm{GeV},
$$

thus the EW scale v can be determined form the EW gauge bosons masses and be extracted for instance from the muon decay rate [41].

The fermions acquire a mass through the Yukawa interactions with the Higgs field. In the unitary gauge the Yukawa Lagrangian reads

$$
-\frac{v+h}{\sqrt{2}}\left[\bar{U}_{L} Y_{U} U_{R}+\bar{D}_{L} Y_{D} D_{R}+\bar{L}_{L} Y_{E} E_{R}+\text { h.c. }\right]
$$

Therefore, once the Higgs boson acquires a vev, and after rotation to the fermion mass eigenstate basis all fermions are given a mass that can be schematically written as

$$
m_{f}=\frac{y_{f} v}{\sqrt{2}},
$$

where $y_{f}$ is the Yukawa coupling for each fermion. Note that the Higgs mechanism does not provide any explanation for the disparity of fermion masses, often referred to as the flavor puzzle.

Finally, the Higgs mechanism solves the loss of unitarity problem in the SM (see Sect. 1.2). For instance, the Higgs contribution to WW scattering (see diagrams 

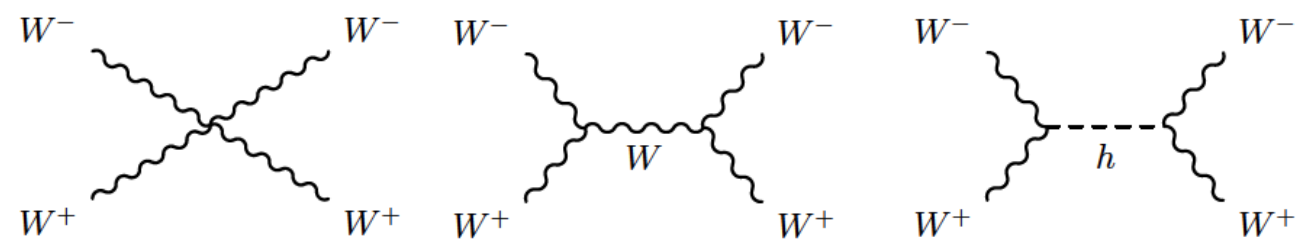

Figure 3.1: Diagrams contributing to the WW scattering at tree level (without crossings). The amplitude from the diagrams in which a Higgs is exchanged adds to Eq.(1.6) to make unitarity be restored.

in Fig. 3.1) precisely provides the necessary contribution to cancel the dependence with the square of the energy that eventually violates unitarity, see Eq. (1.6).

In 2012 a new resonance of mass around $125 \mathrm{GeV}$ was discovered at the LHC (see Fig. 3.2) [1,2], which so far seems consistent with the hypothesis of being a SM Higgs boson. To simplify, we will thus refer to this scalar as the "Higgs boson", $h$, in the remaining part of this thesis.
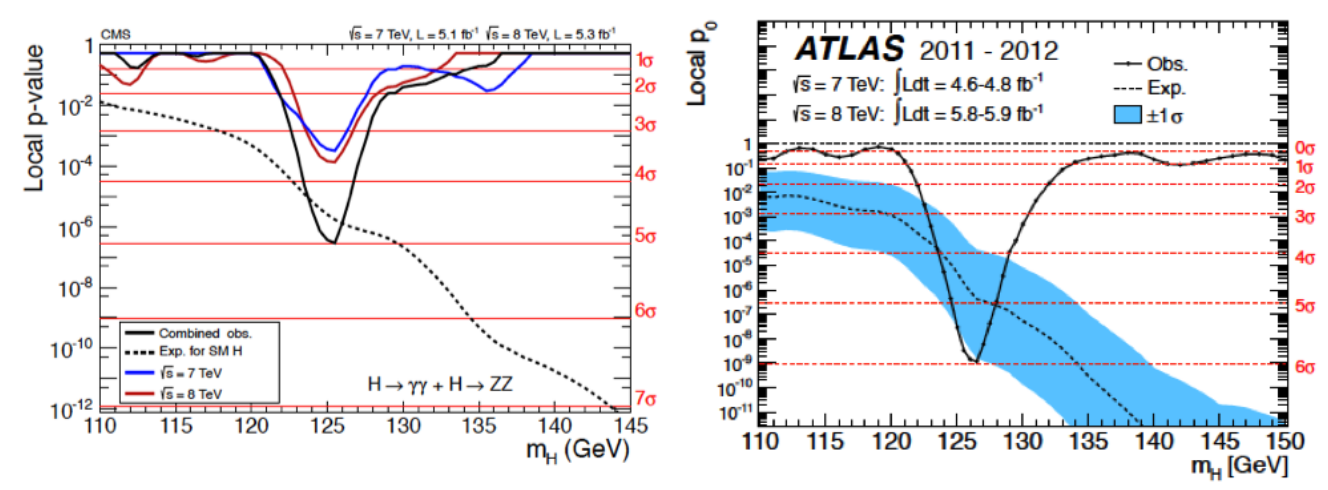

Figure 3.2: The local p-value (solid line) as a function of the Higgs mass observed in the CMS (left) and ATLAS (right) experiments by $2012[1,2]$. The dashed curve is the expected local p-values for having a SM Higgs at a certain value for $m_{H}$. The p-value is a measure of the probability of seeing something at a certain spot against the hypothesis that there is nothing there. The horizontal dashed red lines indicate the p-values corresponding to different significances.

\subsection{SM Higgs boson phenomenology at the LHC: production and decay}

In recent years both Higgs production cross sections and decay branching ratios have all been calculated at high order in perturbation theory. They are shown in Fig. 3.3 with their uncertainties. A summary of the computations can be found in Refs. [51-54]. 

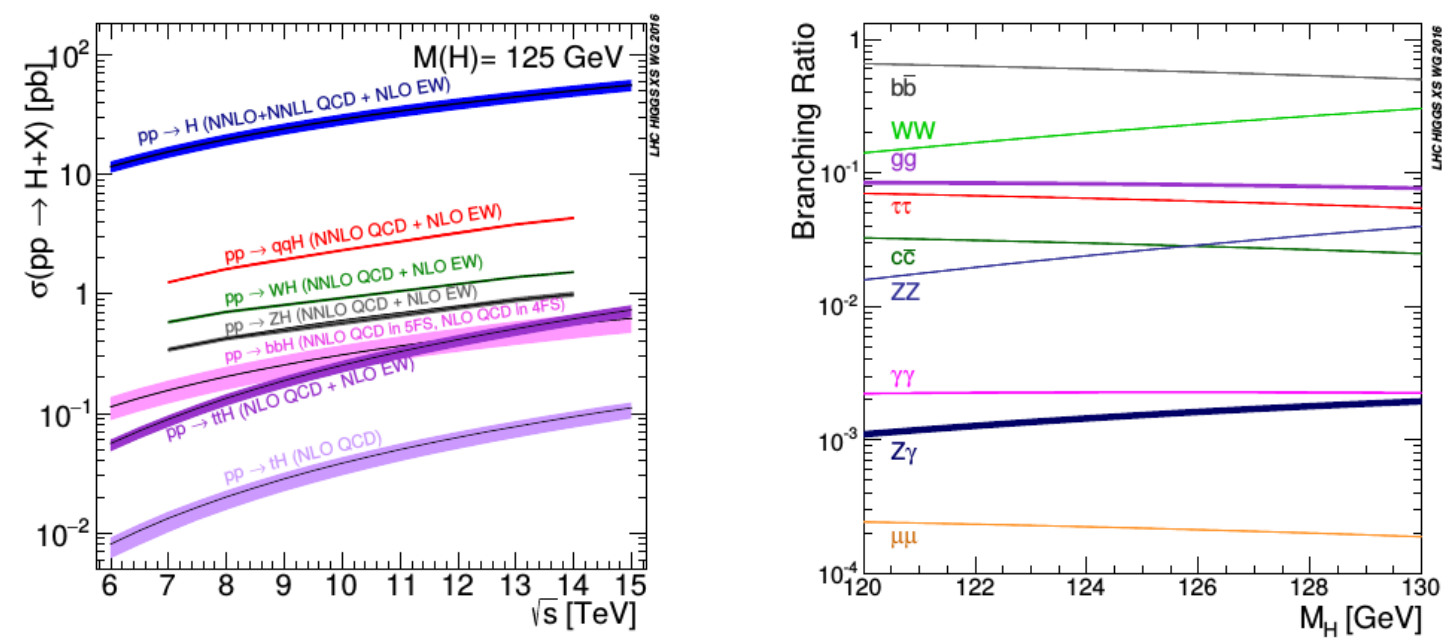

Figure 3.3: SM Higgs computed production cross sections (left) as a function of the center of mass energy for pp collisions and branching ratios (right) for a Higgs boson mass near $125 \mathrm{GeV}$. The theoretical uncertainties are indicated as bands. Figure taken from [41].

The main SM Higgs production channels at the LHC are gluon fusion (ggF), vector boson fusion (VBF) and associated production with vector bosons (VH) or a pair of top quarks (ttH); whose representative Feynman diagrams can be seen in Fig. 3.4. In particular, at LHC the production is dominated by far by gluon-gluon fusion,
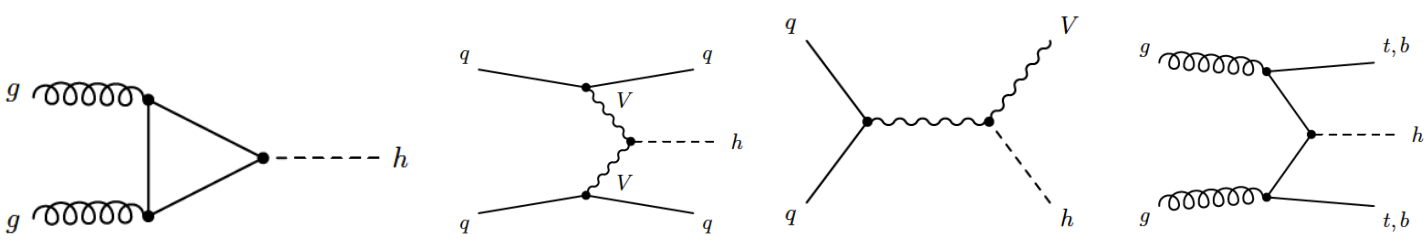

Figure 3.4: Representative Feynman diagrams for the Higgs main production modes at LHC. From left to right: gluon fusion ( $\mathrm{ggF}$ ), vector boson fusion (VBF), associated production with a weak gauge boson $(\mathrm{VH})$ and associated production with a $t \bar{t}$ pair $(\mathrm{ttH})$. V stands for any of the weak gauge bosons, $Z, W^{ \pm}$.

with the largest contribution coming from a top quark running in the loop (since it has the largest Yukawa coupling). Next comes VBF production; but despite being tree-level, it is suppressed with respect to gluon fusion by the weak coupling and the lower pdfs* of the quarks. It has however a very characteristic signature with two forward hard jets from the scattered quarks.

On the other hand, the Higgs decay is dominated by $b \bar{b}$; followed by the decays into $W W^{*}$, gluons, taus and $Z Z^{*}$. Decays into lighter particles are suppressed by their Yukawa couplings and decays into photons or $Z \gamma$ show a low branching ratio due to being loop-induced. However, despite the low decay rate, $h \rightarrow \gamma \gamma$ is one of the channels with best sensitivity for the observation of a Higgs boson at LHC for its high mass resolution and low background. The other relevant channel to observe

\footnotetext{
*Parton distribution functions
} 


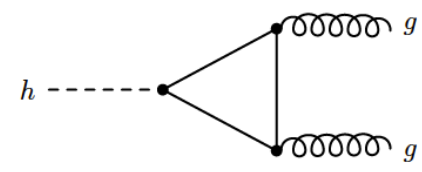

Figure 3.5: Higgs decay into photons occurs through a loop (dominated by tops).

the Higgs is $h \rightarrow Z Z^{*} \rightarrow 4 l$ ( $l$ meaning lepton) because leptons are easily measured and the invariant mass can be reconstructed more easily than in the $h \rightarrow W W^{*} \rightarrow$ $l^{+} \nu l^{-} \bar{\nu}$ case (where neutrinos are present). Finally, the decays involving hadrons are difficult to measure due to the overwhelming QCD background.

\subsection{The Higgs boson from experiment}

The aim of this section is simply to briefly state the main properties of the observed Higgs boson and summarize the current status of the experimental data.

Main quantum numbers: $J^{P C}=0^{++}$

In brief, the discovered neutral particle was found to be a CP-even scalar of spin $J=0[55,56]$. The fact that it was detected for the first time in the decay into two photons $(h \rightarrow \gamma \gamma)$; since that photons are odd under charge conjugation (C), meant that it should be a C-even state. Furthermore, the Landau-Yang theorem ${ }^{\dagger}[57,58]$, states that a massive particle with spin 1 cannot decay into a pair of identical massless spin-1 particles; thus forbidding the observed particle to have $J=1$ from the observation in the diphoton channel. Finally, systematic analyses of different decays $\left(h \rightarrow \gamma \gamma, h \rightarrow W\left({ }^{*}\right) W\left({ }^{*}\right) \rightarrow l \nu l \nu\right.$ and $h \rightarrow Z\left({ }^{*}\right) Z\left({ }^{*}\right) \rightarrow 4 l$ ) yield that the spin-parity of the boson is $J^{P}=0^{+}$(it has spin 0 and even parity), excluding the other possibilities at more than $99 \%$ confidence level $[59,60]$.

\section{Mass}

The SM does not provide a prediction for the mass of the Higgs boson. It is measured from the two channels with best resolution: $\gamma \gamma$ and $Z Z$. The combination from the mass measurements by ATLAS and CMS is [61]

$$
m_{h}=125.09 \pm 0.21 \text { (stat.) } \pm 0.11 \text { (syst.) } \mathrm{GeV}
$$

which is a precision of $2 \%$.

\section{Width}

\footnotetext{
$\dagger$ The Landau-Yang theorem does not apply if the observed state is not decaying to a pair of photons but to a pair of scalars subsequently decaying to two very collimated pairs of photons. However, this possibility is not experimentally favored.
} 
The total width for a $125 \mathrm{GeV}$ SM Higgs boson is rather narrow [41,62]:

$$
\Gamma_{h}^{S M}=\left(4.07 \times 10^{-3}\right)_{-3.9 \%}^{+4.0 \%} \mathrm{GeV} .
$$

See Sect. 3.2 for a brief reminder of the main Higgs decay channels. The total width is the sum of the decay rates for all the allowed decays of the particle. Therefore, finding deviations from the expected value is very important regarding possible new physics beyond the SM. For instance, if $\Gamma_{h}$ was found to be larger, it could indicate the Higgs effective couplings to visible particles are larger than in the SM expectation, or even that the resonance is indeed two quasi-degenerate states.

The Higgs width is still well below the resolutions of LHC experiments, so just constraints for it are available so far. Direct constraints can be obtained from analyses of the reconstructed mass lineshapes in the same decay channels that allow the best mass determination, $\gamma \gamma$ and $Z Z[63,64]$. In the following table the direct constraints at $95 \%$ CL obtained through this method by the two experiments are shown:

\begin{tabular}{ccc}
\hline Channel: & $h \rightarrow \gamma \gamma$ & $h \rightarrow Z Z \rightarrow 4 l$ \\
\hline ATLAS & $<5.0 \mathrm{GeV}$ & $<2.6 \mathrm{GeV}$ \\
CMS & $<2.4 \mathrm{GeV}$ & $<3.4 \mathrm{GeV}$ \\
\hline
\end{tabular}

All these constraints are much larger than the expected SM width and are fully compatible with the SM hypothesis.

On the other hand, the best indirect constraint on the total width is obtained by analyzing diboson (VV) decay channels using simultaneously on-shell measurements (near the resonance peak, where the Higgs propagator is on-shell) and off-shell measurements (away from the resonance peak, where the Higgs propagator is offshell). The best constraint obtained using this method has been determined by ATLAS [65], being $\Gamma_{h}<5.7 \Gamma_{S M} \approx 23 \mathrm{MeV}$ at $95 \% \mathrm{CL}$. This constraint is much stronger than the direct bounds; but it was obtained under the assumption that no new physics alters the Higgs boson couplings in the off-shell regime. CMS has also derived a bound allowing for anomalous couplings of the Higgs boson, giving as result $\Gamma_{h}<10.9 \Gamma_{S M} \approx 44 \mathrm{MeV}$ at $95 \% \mathrm{CL}$ [66].

\section{Couplings to SM particles}

As shown in Eq. (3.20), the SM Higgs boson is expected to be a narrow resonance $\left(\Gamma_{h} / m_{h}\right.$ is small), so it is possible to factorize the production and decay of the Higgs boson in an individual channel:

$$
\sigma(i \rightarrow h \rightarrow \mathrm{f})=\sigma(i \rightarrow h) * B R(h \rightarrow \mathrm{f})=\frac{\sigma(i \rightarrow h) \Gamma(h \rightarrow \mathrm{f})}{\Gamma_{h}},
$$

where $\Gamma_{h}$ is the Higgs total width. $B R$ stands for the branching ratio of the decay, which measures the fraction of events for the particle to decay through a particular channel, so it is given by the ratio between the partial and total decay widths.

Higgs data is usually expressed in terms of the so-called signal strengths. These parameters are defined as the ratio between the observed rate and the SM prediction. 
For a process $i \rightarrow h \rightarrow f$

$$
\mu_{i}^{f}=\frac{\sigma(i \rightarrow h) B R(h \rightarrow f)}{\sigma_{S M}(i \rightarrow h) B R(h \rightarrow f)} .
$$

Using the narrow width approximation as above the individual signal strengths can be factorized:

$$
\mu_{i}^{f}=\mu_{i} \mu^{f}, \quad \mu_{i}=\frac{\sigma(i \rightarrow h)}{\sigma_{S M}(i \rightarrow h)}, \quad \mu^{f}=\frac{B R(h \rightarrow f)}{B R(h \rightarrow f)} .
$$

By definition, all $\mu_{i}^{f}=1$ for a SM Higgs boson. The simplest test of compatibility of the experimental data with the SM expectation is to assume $\mu_{i}=\mu^{f}=\mu$ for all production decay channels; so all SM predictions are scaled by a global signal strength $\mu$. A fit for this from the latest combined data from ATLAS and CMS provides the value [67]

$$
\mu=1.09_{-0.10}^{+0.11}
$$

which is compatible with the SM. The fits to the individual signal strengths for the most relevant production and decay modes are shown in Fig. 3.6.
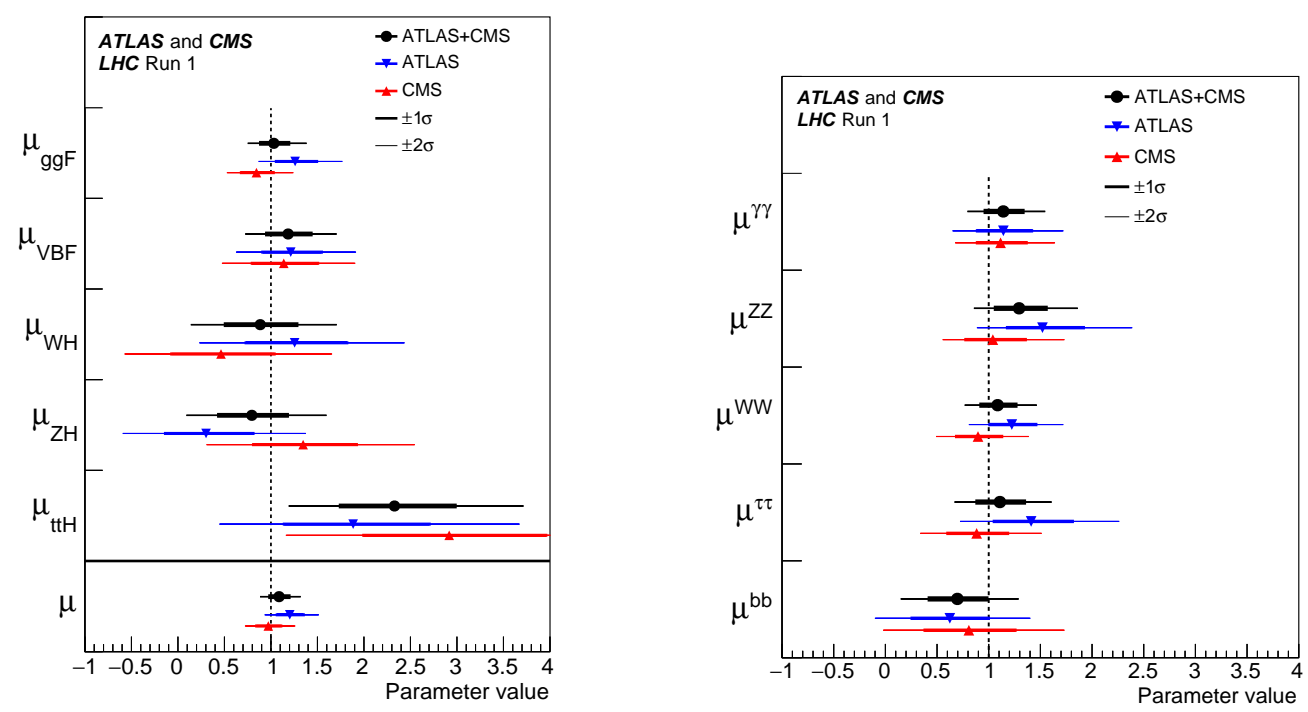

Figure 3.6: ATLAS and CMS fit results for the signal strengths for the different Higgs production (left) and decay (right) channels. On the left plot the measurements for the global signal strength $\mu$ are also shown. Figure taken from [67].

The Higgs couplings are not measured directly, instead it is their ratio with the SM predictions that is determined. Deviations from the SM in the Higgs couplings can be parametrized within the $\kappa$-framework [68]; where some rescaling factors for every production cross-section and decay width of the $h$ particle are introduced:

$$
\kappa_{i}^{2}=\frac{\sigma(i \rightarrow h)}{\sigma_{S M}(i \rightarrow h)} \quad \text { or } \quad \kappa_{\mathrm{f}}^{2}=\frac{\Gamma(h \rightarrow \mathrm{f})}{\Gamma_{S M}(h \rightarrow \mathrm{f})}
$$

in such a way that all $\kappa_{i}=1$ if $h$ is the SM Higgs. This amounts to a rescaling of the couplings of the SM; so in this way it is possible to rewrite the relevant part of 
the Lagrangian for the Higgs production and decay in the following form:

$$
\begin{aligned}
\mathcal{L}_{h}= & -\sum_{f} \kappa_{f} \frac{m_{f}}{v} \bar{f} f h+\kappa_{Z} \frac{m_{Z}^{2}}{v} Z_{\mu} Z^{\mu} h+\kappa_{W} \frac{m_{W}^{2}}{v} W_{\mu}^{+} W^{\mu-} h \\
& +\kappa_{g} \frac{g_{s}^{2}}{48 \pi^{2} v} G_{\mu \nu}^{A} G^{A \mu \nu} h+\kappa_{\gamma} \frac{e^{2}}{16 \pi^{2} v} A_{\mu \nu} A^{\mu \nu} h+\kappa_{Z \gamma} \frac{e^{2}}{4 \pi^{2} v} A_{\mu \nu} Z^{\mu \nu} h .
\end{aligned}
$$

It is clearly seen in this equation that the main limitations from the $\kappa$-framework are that there are no different Lorentz structures than in the SM and that the Lagrangian is not explicitly invariant under the whole SM gauge symmetry, although it does respect the electromagnetic gauge group. Therefore it is just suited to treat deviations from the SM at leading order ${ }^{\ddagger}$; but it works well in fits based on total rates. Interference effects from different subprocesses can provide some sensitivity to relative signs between different Higgs couplings.

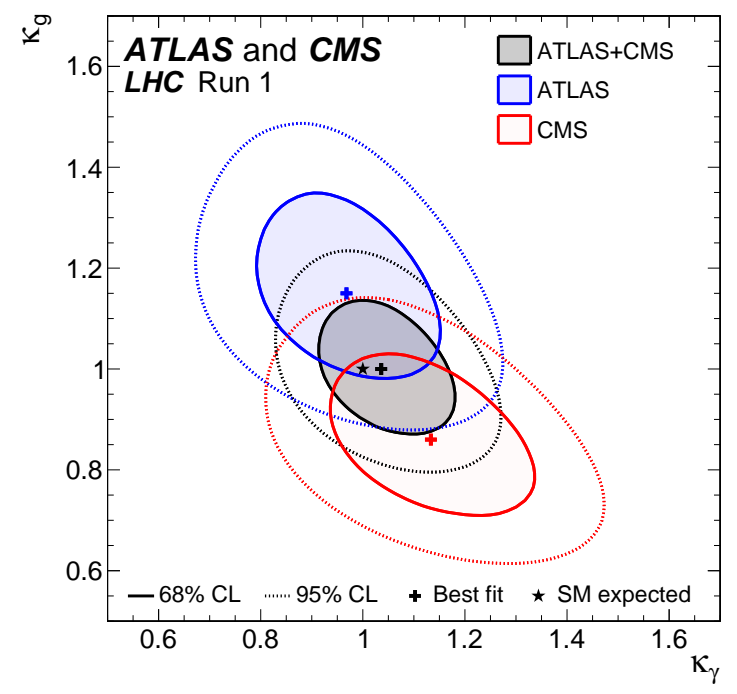

Figure 3.7: Negative log-likelihood contours at $68 \%$ and $95 \%$ CL in the $\left(\kappa_{g}, \kappa_{\gamma}\right)$ from ATLAS and CMS experiments. The fit is obtained by constraining all other coupling modifiers to 1 and assuming no BSM contribution to the Higgs width. Figure taken from [67].

The last three terms in (3.26) are effective couplings generated at one-loop in the SM. Fig. 3.7 shows the fit to $\kappa_{g}$ and $\kappa_{\gamma}$ assuming there is no BSM contribution to the $h$ couplings to SM particles, so the presence of BSM resonances in the loop can be tested. It is found that the SM point $\left(\kappa_{g}=\kappa_{\gamma}=1\right)$ lies within the $68 \% \mathrm{CL}$ contour and the p-value of the compatibility with the SM is $82 \%$ [67]. This makes the assumption that there are no BSM particles in those loops a reasonable one, as it is done in the fit shown in Fig. 3.8. Both fits in that figure show that the experimental data is generically consistent with the SM expectation; although the error bars are still quite large.

The Higgs boson couplings to SM particles depend on their masses. In particular, Higgs couplings to fermions depend linearly on their masses and are flavor diagonal;

${ }^{\ddagger}$ Most dominant higher-order QCD corrections factorize though. 


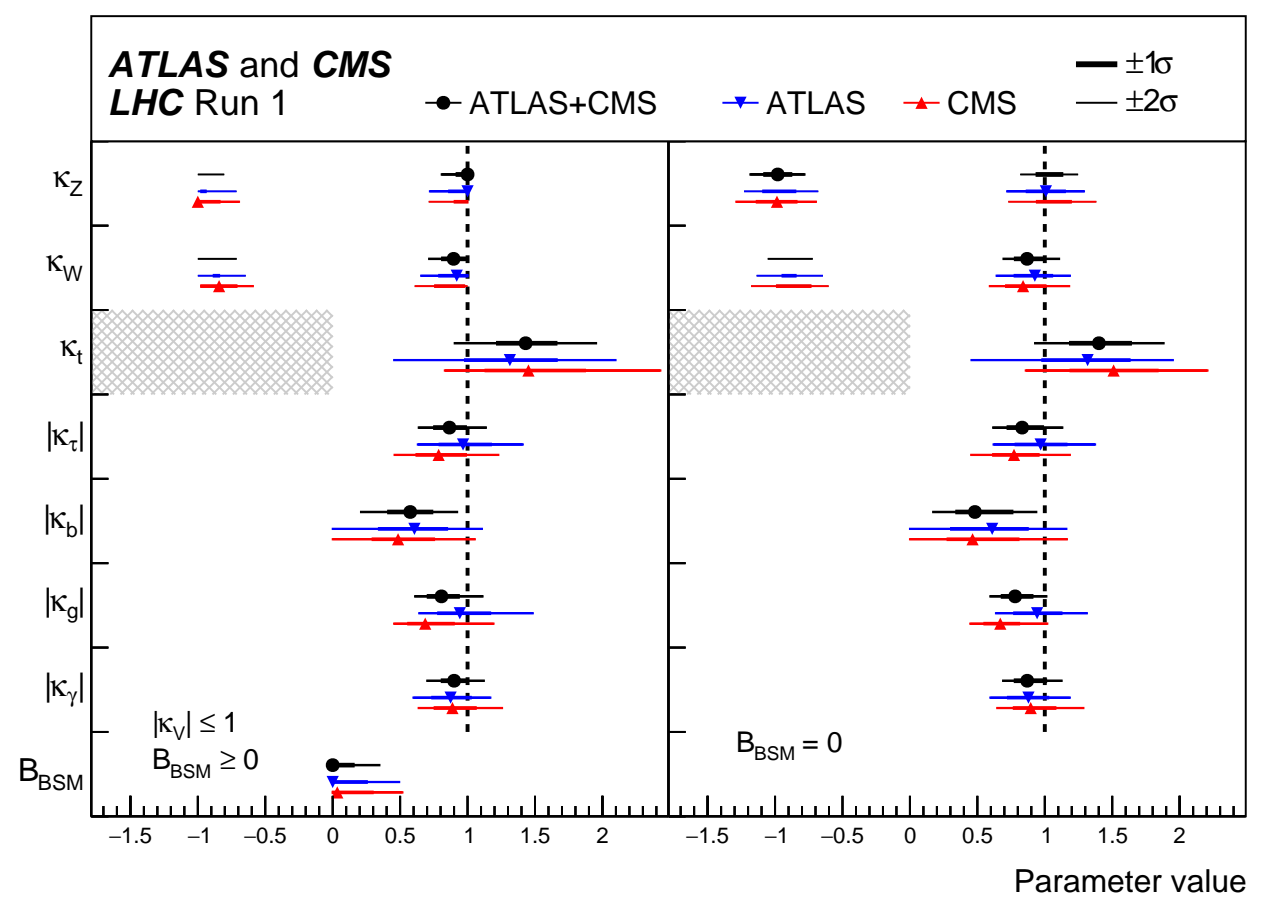

Figure 3.8: ATLAS and CMS fit results for the coupling modifiers both allowing (left) and not allowing (right) BSM contributions $\left(B_{B S M}\right)$ to the Higgs width. The hatched areas show the non-allowed regions for $\kappa_{t}$, which is assumed to be positive without loss of generality. Figure taken from [67].

while couplings to the $\mathrm{W}$ and $\mathrm{Z}$ bosons are proportional to the square of their mass (see Sect. 3.1). Fig. 3.9 shows the fit for the measured couplings as a function of the particle masses [67]. This way it can be clearly seen how the couplings scale with the particle mass as in the SM expectation (see dashed line on the plot).

As some couplings are very weak (Higgs couplings to the lighter fermions or its own self-couplings), it is very challenging to probe them and so they are missing in the fits shown here. There are several other fits to study the compatibility of the Higgs couplings with the SM; see Refs. [41,67,70].

\subsection{Triviality and Stability}

It is possible to compute how the quartic coupling of the Higgs potential ( $\lambda$ in Eq. (3.5)) runs with the energy scale ${ }^{\S}$. It turns out that within the values in the SM for the Higgs couplings, $\lambda$ remains perturbative all the way up to the Planck mass. For large values of $\lambda$, the running with energy is approximately

$$
\lambda(\mu) \sim \frac{\lambda\left(\mu_{0}\right)}{1-\frac{3 \lambda\left(\mu_{0}\right)}{4 \pi^{2}} \log \left(\frac{\mu}{\mu_{0}}\right)},
$$

\footnotetext{
§See for instance Ref. [71] for a computation of the SM renormalization group equations, with the Higgs self-coupling being one of them.
} 


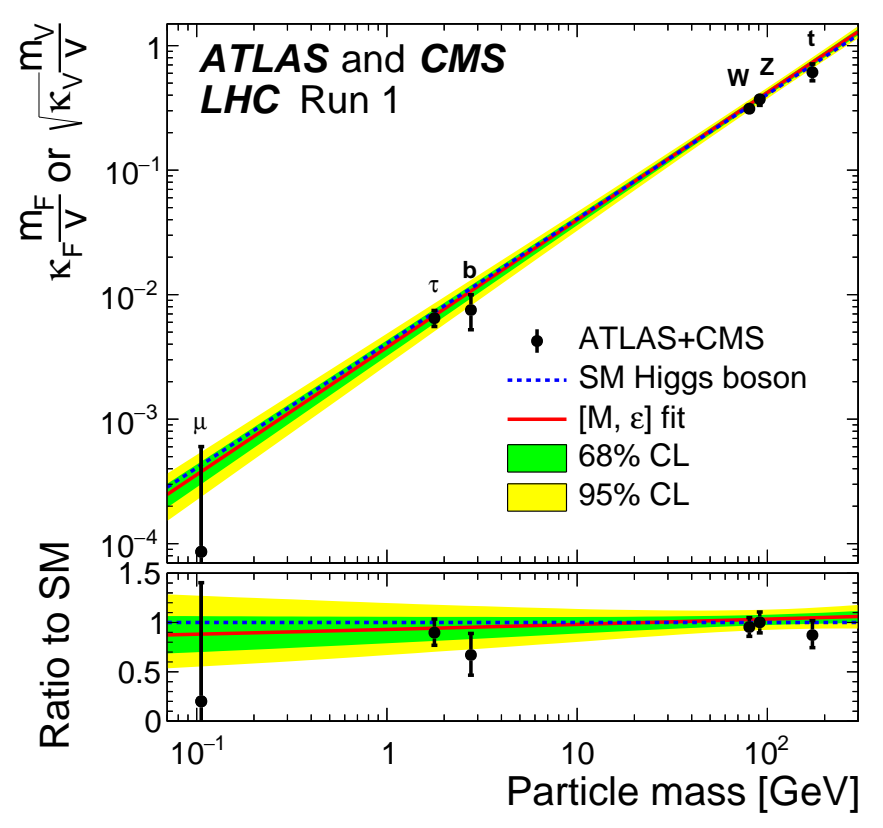

Figure 3.9: Best fit values of the Higgs couplings as a function of the particle mass from the combination of ATLAS and CMS data. Yukawa couplings are defined as $\kappa_{F} m_{F} / v$ for fermions, while $\sqrt{\kappa_{V}} \frac{m_{V}}{\sqrt{v}}$ is the reduced coupling for the weak gauge bosons, where $v=246 \mathrm{GeV}$ is the Higgs vacuum expectation value. The $[M, \epsilon]$ fit is explained in [69]. The dashed (blue) line indicates the SM prediction and the ratio of the reduced couplings to the SM expectation is shown in the bottom panel. Figure taken from [67].

where $\mu$ is the energy scale and $\mu_{0}$ is some fixed energy scale. All gauge and Yukawa contributions have been neglected (which is a reasonable approximation for large $\lambda)$. From the equation it can be seen that the coupling would grow with $\mu$ until it will eventually hit a pole at

$$
\tilde{\mu}=\mu_{0} \exp \left(\frac{4 \pi^{2}}{3 \lambda\left(\mu_{0}\right)}\right) .
$$

$\tilde{\mu}$ might be pushed to infinity, while fixing $\lambda\left(\mu_{0}\right)$ to a finite value; so it is equivalent with taking the limit $\mu_{0} \rightarrow \infty$ in Eq. (3.27) so the effective coupling $\lambda(\mu) \rightarrow 0$ and thus the theory is non-interacting or, in other words, trivial. A way out of this is to assume the existence of a physical cut-off, for instance the Planck mass, $M_{P} \approx$ $1.2210^{19} \mathrm{GeV}$, and fix $\mu_{0}=v=246 \mathrm{GeV}$, the EW energy scale. This provides an upper bound on the coupling that translates into the Higgs mass $m_{h}^{2}=\lambda(v) v^{2}$ :

$$
\lambda(v)<\frac{4 \pi^{2}}{3 \log \left(\frac{M_{P}}{v}\right)} \Rightarrow m_{h}^{2}<\frac{4 \pi^{2} v^{2}}{3 \log \left(\frac{M_{P}}{v}\right)} \approx(144 \mathrm{GeV})^{2} .
$$

Since the Higgs boson has been found to be quite light (see previous section), this bound is satisfied. Besides, the limit gets less restricted as the cut-off decreases and it assumed a large $\lambda$ (large Higgs mass) from the start.

Indeed, in the SM, with a Higgs mass of $125 \mathrm{GeV}$ and the EW scale $v=246 \mathrm{GeV}$, the quartic coupling is rather small, $\lambda=0.13$. For small $\lambda$, the main contributions 

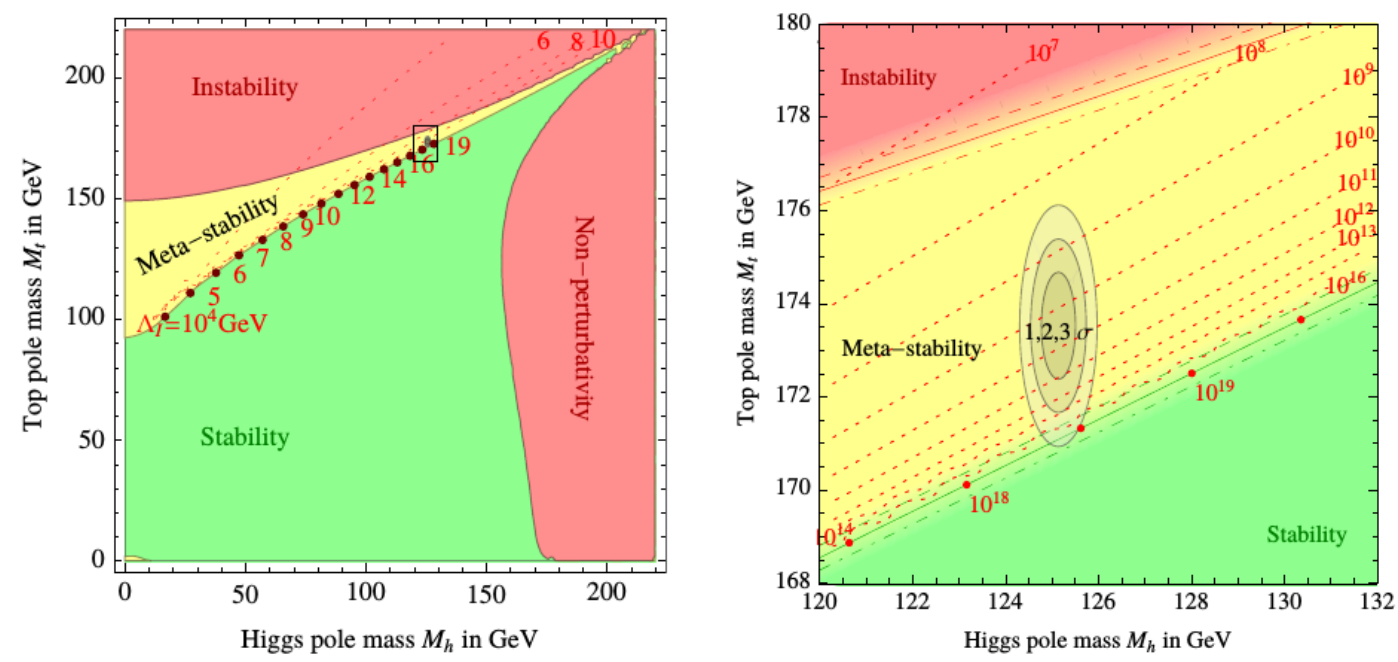

Figure 3.10: Stability, meta-stability and instability regions of the SM vacuum in terms of Higgs and top pole masses. On the right zoom in the meta-stability region showing the $1 \sigma, 2 \sigma$ and $3 \sigma$ regions of the preferred experimental range of the Higgs and top pole masses. Figure taken from [72].

to its Renormalization Group Equation (RGE) - i.e. its running with the energy scale - come from the weak gauge bosons and the top quark:

$$
\mu \frac{d \lambda}{d \mu} \approx \frac{3}{4 \pi^{2} v^{4}}\left(2 m_{W}^{4}+m_{Z}^{4}-4 m_{t}^{4}\right) .
$$

In particular the top quark contribution eventually wins at large energies, making $\lambda$ negative, so the potential becomes unbounded from below.

In the SM the $\lambda$ coupling becomes negative at around $10^{10} \mathrm{GeV}$ [72-75]. It actually turns out that the SM parameters are precisely such that they keep the SM in meta-stability (see Fig. 3.10); i.e. the SM vacuum is just a local one and at larger energies there is the global one to which the SM can decay through tunneling effects. The tunneling probability is proportional to $e^{-1 / \lambda}$, and $\lambda$ only gets slightly negative with the SM parameters, so the EW vacuum would remain stable with an expected lifetime much larger than the age of the Universe. Still, all this analysis relies on the assumption that there is no new physics anywhere up to the Planck scale. 


\section{Chapter 4}

\section{Having a light Higgs}

\subsection{Naturalness and the "Hierarchy Problem"}

The notion of naturalness in particle physics is often formulated in terms of the definition given by t'Hooft [76]:

"At any energy scale $\mu$, a physical parameter or set of physical parameters $\alpha_{i}(\mu)$ is allowed to be very small only if the replacement $\alpha_{i}(\mu)=0$ would increase the symmetry of the system".

There has been occasions in the past where naturalness has been a guide for new physics. A beautiful example of this is the prediction of the charm quark mass: in Ref. [77] a quite accurate estimation of the charm quark mass was proposed in order to explain the suppression of flavor-changing neutral currents in kaon physics through the GIM mechanism [78].

The so-called Hierarchy Problem (HP) can be stated as the failure of the SM to provide a natural explanation for the lightness of the Higgs particle. The technical reason for this is that when loop corrections of the self-energy of the Higgs scalar are computed (the result obtained is proportional to the UV cut-off scale squared $\left(\Lambda^{2}\right)$. Then the bare Higgs mass, $m_{0}^{2}$ has to be big enough to cancel the corrections, $\Delta m_{H}^{2}$, to yield the physical mass

$$
m_{h}^{2}=m_{0}^{2}+\Delta m_{H}^{2}\left(\Lambda^{2}\right) .
$$

Therefore, there would not be a problem if the SM, which is a renormalizable theory, does not break down at any scale and there is no NP coupled to the Higgs. However, there are good reasons to think there has to be NP somewhere, since it is indicated by observations outside the SM, as it was introduced in the Motivations to this thesis.

In conclusion, the hierarchy problem appears whenever there is NP with particles of mass $\Lambda$ that couple to the Higgs, as the associated loop corrections to its self-energy 
will push its mass up to the new scale by terms of order $\Lambda^{2}$. This means that the Higgs is sensitive to any beyond the standard model (BSM) scale coupled to it, and it is actually very easy to construct operators containing a scalar via terms with $|\phi|^{2}$ coupled to any kind of new particle (for instance, right-handed neutrinos as in type I seesaw).

Eq. (4.1) then points to a fine-tuning problem, depending on the gap between $\Lambda$ and $v$, the EW scale. The fine-tuning manifests itself only in the corrections to the elementary scalar in the theory, and not to any other particle of the SM, due to the gauge symmetry that is protecting their masses; i.e. the gauge symmetry protects explicit mass terms for fermions and gauge bosons in the Lagrangian, while a mass term for a scalar boson is allowed. Note that it might be argued that there are renormalization schemes in which the hierarchy problem can be avoided, as, for instance, dimensional regularization, where there is no cut-off dependence; but this reasoning is misleading. Indeed, the hierarchy problem is much more than a question of an absorption of loop divergences; it has to do with the lack of a natural explanation for the huge gap in energy scales between EW physics and any New Physics (NP). Or put in other words, it has to do with the unnaturalness (i.e. fine-tuning) in the parameters of the low energy theory (the SM), which should be reproduced by a NP superseding theory at higher scales.

Of course, the hierarchy problem might be regarded as only a theoretical consideration, and maybe we just should learn to live with fine-tunings in the parameters and abandon the idea that they are indications calling for new physics. However, as in the case of the charm quark mentioned above, naturalness issues could point to new physics and in this sense it is considered a worthy discussion.

A possible solution to the hierarchy problem is to introduce a symmetry that protects a mass term for the $h$ scalar; just as fermion and gauge boson mass terms are protected in the SM model by the gauge symmetry. We will come back to them later.

There are also possibilities without implementing new symmetries. For instance, it is possible to justify the HP through anthropic arguments: an EW vev at around $246 \mathrm{GeV}$ can be explained from baryon stability [79]. Therefore, assuming a selfcoupling of the order of the SM one, the Higgs is found to be light because that is the one having the right properties for our existence; so fine-tuning is no longer a problem. However, that explanation is at the expense of assuming that there are different values of the physical parameters in different regions of the universe (or in different parallel universes).

Another possibility, which was proposed very recently [80] - see also Ref. [81], is the so-called relaxion mechanism. In this theory the SM Higgs couples to a new field, the relaxion, so the Higgs mass depends on the value of this field. As shown in Fig. 4.1, the relaxion has a potential with many local minima; so that at present times it is sitting on one of those minima, such that the Higgs mass has the observed value. However, one has to find a suitable candidate for the relaxion particle and another recent work [82] has discussed whether a relaxion model is consistent in its simplest implementation. 


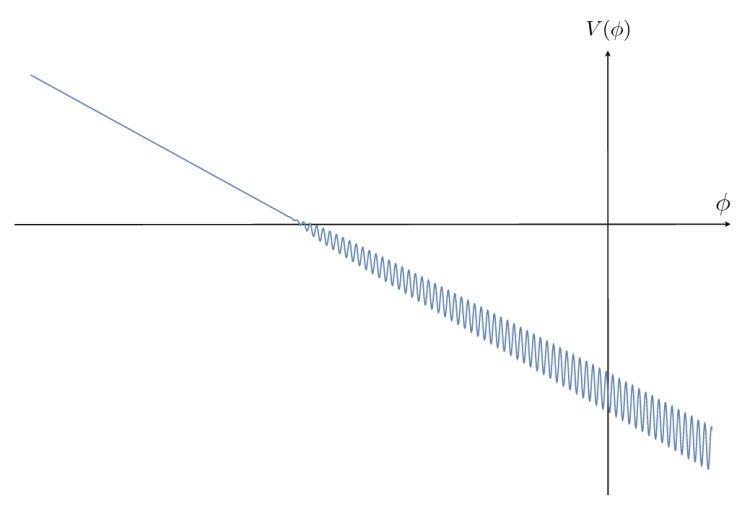

Figure 4.1: The potential for the relaxion field, $\phi$, which rolls down during inflation until the EWSB stops the time dependence and the EW scale is fixed and the relaxion field stays at one of the minima. From Ref. [80].

Two main approaches study the possibility of a symmetry protecting the Higgs mass: low-scale supersymmetry (SUSY) and the Higgs as a pseudo-Goldstone boson (pGB). In SUSY there are two Higgs doublets and each particle in the SM is accompanied by a superpartner (an sparticle), which contributes to the Higgs self-energy with opposite sign with respect to that of the SM particle. Indeed, if the SUSY symmetry is not broken, the superpartners have the same mass and the whole correction to the Higgs mass vanishes.

Furthermore, supersymmetry has very interesting properties; since it makes possible for the strong, weak and hypercharge gauge couplings to unify at high energies and also the lightest supersymmetric particle (LSP) as it is usually predicted in SUSY models constitutes a good candidate for cold dark matter (it is stable due to Rparity, neutral and has the right relic abundance). Supersymmetry also have other very interesting properties motivated by some theoretical problems. For instance, it allows to combine the gauge interactions with gravity within the supergravity framework.

Nevertheless, no SUSY particle has been seen so far. Actually, no particle associated with NP has been detected at the LHC yet, which seems to point to a gap in the spectrum. The non-observation of superpartners also means that supersymmetry must be broken somehow. If it turns out that the masses of the new particles are too heavy, there might still be a fine-tuning problem. Therefore, it is argued that the scale of a supersymmetric model to explain the hierarchy problem, at least in its simplest implementations, should not be too far away from the TeV scale.

As it was stated in the Motivation for this thesis, our focus has been on the study of the implications of the pseudo-GB possibility. This possibility has the interesting feature of providing a natural energy gap, as it will be explained below; although it is not free from fine-tunings either. To introduce the idea, which will be done in Sect. 4.3, it is instructive first to revise the possibility of describing EWSB without a Higgs particle. 


\subsection{Higgsless EWSB}

As it was briefly introduced in Sect. 2.2.2, in the non-linear sigma model it is possible to describe the spontaneous chiral symmetry breaking of QCD chiral symmetry by means only of the GB degrees of freedom, at the expense of losing renormalizability. This was the inspiration for technicolor models, which began to be developed in the late 1970s [83-87].

Let us consider again QCD with two flavors of massless quarks, so it has a $S U(2)_{L} \times$ $S U(2)_{R}$ chiral symmetry; which is broken by quark-antiquark condensates producing three pions as GBs. When the $S U(2)_{L} \times U(1)_{Y}$ interactions are turned on, the EW gauge bosons will couple to the three broken axial currents, "eating" the pions as it is expected to happen when a local symmetry is broken spontaneously (see Sect. 2.3). The pions have thus become the longitudinal components of the gauge bosons and therefore are no longer present in the spectrum. The gauge bosons have then acquired a mass of order $g f_{\pi}$ where $g$ is the corresponding gauge coupling and $f_{\pi}$ is the pion decay constant (the energy scale of the GBs). However, since the pion decay constant is measured to be $f_{\pi} \approx 92 \mathrm{MeV}$, this mass is far too low, just around $30 \mathrm{MeV}$. See Refs. [88, 89] for a good pedagogical description of EWSB induced through QCD chiral symmetry spontaneous breaking.

One can then try to extend this and add to the model a $S U\left(N_{T C}\right)$ technicolor gauge sector with a global $S U(2)_{L} \times S U(2)_{R}$ symmetry. Let us assumed this is a confining theory, analogously to QCD, so condensates of "techniquarks" break the chiral symmetry spontaneously producing at least $3 \mathrm{GBs}$. The new GBs energy scale can now be pushed upwards to be the EW scale, $F_{\pi} \simeq v=246 \mathrm{GeV}\left(v^{2}=f_{\pi}^{2}+F_{\pi}^{2}\right.$, $f_{\pi} \ll F_{\pi}$ ); a value that can provide the right values for the masses of the $W^{ \pm}$and $Z$ gauge bosons. In this context it is a linear combination of the standard pions and the new technipions that becomes the longitudinal components of the gauge bosons. But since $f_{\pi} \ll F_{\pi}$, the longitudinal components of the gauge bosons will mostly be the technipions; while the physical pions will mostly be the QCD pions.

In its simplest implementations, there is no light Higgs scalar in the technicolor spectrum. As commented in Sect. 1.2, in such an scenario unitarity is lost and the EWSB sector is strongly-interacting at high energies. Nevertheless, unitarity in the scattering of longitudinal gauge bosons is restored due to the contribution from a tower of new composite resonances. The strength of the interaction would indeed grow with energy; but as the energy is high enough for the composite resonances to start being produced and contribute to the scattering, the effective theory breaks down and the unitarity bound is not violated. The spectrum would in fact be like a heavier replica of that of QCD. Thus the most relevant contribution is expected to be that of the lightest resonance (the "technirho") and its mass can be estimated in terms of that of the $\rho$ meson in QCD $\left(m_{\rho} \approx 775 \mathrm{MeV}\right)$ by means of the large-N approximation [90-92]:

$$
m_{\rho}^{T C} \sim \frac{F_{\pi}}{f_{\pi}} \sqrt{\frac{N_{C}}{N_{T C}}} m_{\rho} \approx 1.8 \mathrm{TeV}, \quad N_{T C}=4,
$$

so the new resonances are expected to appear at the TeV. Therefore, technicolor 
solves the hierarchy problem of the EW scale because it is generated in such a way that it can have much lower values than the typical resonances of the $S U\left(N_{T C}\right)$ theory.

However, most simple technicolor models are plagued by flavor-changing neutral currents (FCNC); which depends on the implementation of quark masses in the model and thus is rather model-dependent. Moreover, they are strongly constrained by electroweak precision observables (EWPO), in particular by the so-called PeskinTakeuchi parameters S and T [93-95].

\subsection{The Higgs as a pseudo-GB}

There is a way to construct a hybrid theory between technicolor and elementary Higgs models. This was proposed by Georgi and Kaplan in the eighties with a $S U(5)$ symmetry spontaneously broken to $S O(5)$ [7], see also Refs. [7,96-99]. This theory is an example of composite Higgs models. The aim of this section is to revise the main features of these models, as they are an excellent example of why they provide a way to evade the hierarchy problem, before going on to comment briefly on different modern approaches; which serves as the final part of the prelude to present the work done for this thesis.

In the theory of Georgi and Kaplan, it is assumed there is some strong dynamics given by an "ultracolor" theory with some global symmetry. The ultracolor theory is assumed to confine, so, just as the pions in QCD, the Higgs particle, $h$, arises as a bound state, at a lower scale than the ultracolor confinement scale because it is a pGB, associated to the spontaneous breaking of the global symmetry by the ultrafermion-antiultrafermion condensate.

Now, the main difference of these models with respect to technicolor is that the ultracolor interaction does not directly break $S U(2)_{L} \times U(1)_{Y}$. At this level, the gauge bosons, together with the light fermions remain massless still. Also the Higgs particle remains massless, as any of the other GBs. Therefore, an independent source of breaking of the EW symmetry has to be introduced at a lower scale, which should also break explicitly the global chiral symmetry. This provides an effective potential for the $h$ field, which triggers EWSB and thus provides mass both to the EW gauge bosons and the Higgs particle, differentiating it from the other GBs by making it a pGB instead.

In consequence, these models are characterized by the existence of separate energy scales in the theory. On the one hand, there is the characteristic scale of the ultracolor theory, $\Lambda$, around which the tower of resonances is expected to appear. On the other hand, below, there is the characteristic scale of the GBs of the theory (which include the Higgs particle), $f$. These two scales are separated by a gap, alike the gap between the light mesons and the other heavier resonances that exists in QCD, with

$$
\Lambda \lesssim 4 \pi f
$$

see Ref. [100]. 
The vev of the Higgs field, $\langle h\rangle$, i.e. the scale at which the EWSB occurs. Finally, $v$, the EW scale, appears here as a fine-tuning introduced to obtain the right masses for the $W^{ \pm}$and $Z$ gauge bosons. It is thus possible that $\langle h\rangle$ and $v$ do not coincide; but there is always a model-dependent relation that links together $f,\langle h\rangle$ and $v$. The ratio

$$
\xi=\frac{v^{2}}{f^{2}}
$$

parametrizes the degree of non-linearity of the Higgs dynamics. It is important for phenomenology as it enters, for example, in the Higgs couplings, so the fine-tuning can be easily constrained from experimental data (see Refs. [32,33,101] and also Sect. 9.1). It takes values between 0 and 1 . When $\xi \rightarrow 0$ it refers to the linear regime, since it corresponds to $f \rightarrow \infty$, i.e. $\Lambda \rightarrow \infty$, so it is equivalent to recovering the SM, as there would be no way to distinguish the composite Higgs from an elementary one. On the other hand, $\xi=1$ refers to the non-linear regime, as $f=v$ makes the extreme case of technicolor models.

The $S U(5) \rightarrow S O(5)$ breaking pattern mentioned at the beginning of this section implies that 24 generators are broken, so 24 GBs should appear as a result. Three of them are needed to be absorbed in order to become the longitudinal components of the EW gauge bosons, and one will turn out in the end to be the Higgs pseudo-GB, so all the rest need to be given a heavy mass, so they do not affect low energy phenomenology. For this reason, most successful recent composite Higgs models implement a $S O(5) \rightarrow S O(4)$ breaking instead (see Refs. [30,102]); so only the necessary minimal amount of GBs (four) are produced. Models considering a $S O(6) \rightarrow S O(5)$ [103] are also interesting because the extra pseudo-GB can provide a dark matter candidate, and because it is isomorphic to $S U(4) \rightarrow S p(4)$; interesting in terms of a possible UV completion with a purely fermionic gauge theory [104-106].

The gauging of the SM symmetry group is not enough to induce the explicit breaking; but in many of these models (for instance, in [30] or see Sect. 8.2 and other references therein), the gauging of the EW symmetry is combined with the inclusion of linear couplings between the heavy resonances and the SM fermions; giving rise to a scenario alike partial compositeness [35]; since as a consequence the SM physical particles emerge after the diagonalization to the mass basis as a superposition of the SM original fields and the new heavier states.

These models avoid the hierarchy problem mentioned above, since the Higgs, being a pGB, has a mass that is protected by the global approximate symmetry against quantum corrections. Furthermore, they provide a natural energy gap; which has become an interesting feature, since the discovery of the Higgs particle has not been accompanied so far by any sign of any other new exotic resonance. However, significant fine-tunings seem to plague composite Higgs models, coming from EWPO or because they quite generally require resonances associated with the top quark (top partners) not to be far from the $\mathrm{TeV}$ scale $[32,33,107-110]$. Also custodial symmetry breaking (see Sect. 5.2) imposes restrictions on these models, so realistic possibilities need are forced to include a custodial symmetry in order to protect for instance the Zbb coupling [111]. 
Some UV completions of composite Higgs models in the market involve an extra dimension in the context of Randall-Sundrum scenarios [112] and make use of the AdS/CFT correspondence; which relates a weakly coupled model of gravity in Antide Sitter spacetime (AdS) in 5 dimensions with a strongly coupled Conformal Field Theory (CFT) in 4 dimensions; see for instance Refs. [30,113,114].

Other examples with a Higgs boson realized as a pseudo-GB are little Higgs models [115-117]. As Georgi explains in [118], the problem in the original models was that it was necessary to introduce two independent sources of breaking of the global symmetry so they contribute differently to the mass and the quartic coupling of the Higgs particle, and thus prevent the Higgs vev from being large. Little Higgs models manage to bypass this by including two (or more) symmetry groups in such a way that each of the associated couplings preserve a symmetry leaving the Higgs as an exact GB; while they break completely the symmetries protecting the Higgs when they act together, and hence this technique is given the name of collective symmetry breaking. In this way they ensure that quadratically divergent contributions to the Higgs mass do not arise at one loop.

In the second part of this thesis an effective field approach is discussed to analyze the implications of a Higgs as a pseudo-GB; while in the third and final part of the thesis a simple toy model and its connection with effective field theory is described. In this model, no composite dynamics is explicitly assumed or described; so it can be used either as a theory of elementary fields or an effective model with a cut-off representing some strong dynamics through a $\sigma$ particle like that of the linear sigma model of QCD. 


\section{Part II}

\section{Higgs Effective Field}

Theory (HEFT) 



\section{Chapter 5}

\section{Effective Lagrangians}

\subsection{Generalities of EFTs}

Without specifying a specific model, there is still useful information about new physics that one can extract by making use of an effective approach. This exploits the fact that, usually, in order to describe the physics at a particular energy scale, one does not need to know the details of physics at other scales; being enough just to keep the relevant degrees of freedom at the scale of interest.

Indeed, Effective Field Theories (EFTs) have been a successful tool in the past and probably the best known example is the Fermi theory of beta decay. Let us review it briefly. It is based on an operator constructed as a contact interaction between the fermions involved in the decay $\left(n \rightarrow p e^{-} \bar{\nu}_{e}\right)$ :

$$
\mathcal{L}_{F e r m i}=-\frac{G_{F}}{\sqrt{2}} J^{\mu} J_{\mu}^{\dagger}
$$

where the strength of the interaction is parametrized with $G_{F}$, the Fermi constant, and the current contains both the quarks and leptons $J_{\mu}=\sum_{i j} \bar{u}_{i} \gamma_{\mu}\left(1-\gamma_{5}\right) V_{i j} d_{j}+$ $\sum_{l} \bar{\nu}_{l} \gamma_{\mu}\left(1-\gamma_{5}\right) l$, with $V_{i j}$ the Cabibbo-Kobayashi-Maskawa mixing matrix.

We know nowadays that the Fermi theory is a low energy theory of the weak interactions. Actually, it is easy to obtain it from the underlying theory, $S U(2)_{L} \times U(1)_{Y}$. At low energies (as compared to the cut-off of the Fermi theory, the EW scale) physical $W$ bosons are not produced. In this case, a virtual $W$ boson is exchanged in the process. Since the momentum transferred is small compared to the $W$ mass, an expansion of the propagator can be performed:

$$
\frac{-g_{\mu \nu}+q_{\mu} q_{\nu} / M_{W}^{2}}{q^{2}-M_{W}^{2}} \quad \underset{q^{2} \ll M_{W}^{2}}{\vec{M}_{W}^{2}} \quad \frac{g_{\mu \nu}}{M_{W}^{2}}
$$

Plugging this in the computation of the amplitude of the process with the full EW theory, the four-point interaction of Eq. (5.1) is obtained. This yields a relation for 
the effective Fermi constant by matching with the high energy parameters:

$$
\frac{G_{F}}{\sqrt{2}}=\frac{g^{2}}{8 M_{W}^{2}} .
$$

Expanding further the $\mathrm{W}$ boson propagator, higher order corrections to (5.1) can be obtained.

In general, an effective field theory is formulated via an effective Lagrangian

$$
\mathcal{L}=\sum_{i} c_{i} \mathcal{O}_{i}
$$

consisting in a set of operators, $\mathcal{O}_{i}$, constructed from the low energy fields. The operators are accompanied by a set of arbitrary coefficients, $c_{i}$, which encode the high energy physics and have to be determined phenomenologically. The tower of operators is usually organized according to their dimension, $\left[\mathcal{O}_{i}\right]=d_{i}$, as this fixes the dimension of the associated coefficient, depending on the powers of the cutoff scale, $\Lambda$, needed to suppress it so all the terms in the Lagrangian have mass dimension four:

$$
c_{i} \sim \frac{1}{\Lambda^{d_{i}-4}}
$$

Therefore, there is a finite set of parameters to compute all the physical quantities at a characteristic energy $E$ at each order, $k=d_{i}-4$, in the effective expansion. Thus the error made by including interactions up to order $k$ is of the order $(E / \Lambda)^{k}$. For this reason, EFTs typically yield best results when the separation between the scale of interest and the scale of the NP, i.e. the cutoff, is large. Increasing the energy means that the non-renormalizable interactions will become more and more important, until the effective theory eventually breaks down.

Finally, the effect from the high-energy theory is not only in the coefficients of the effective expansion, but it also constrains the structure of the low-energy theory from the symmetries it imposes on it, as it will be seen in the following section.

\subsection{The Chiral Effective Lagrangian}

In the case of theories with spontaneously broken symmetries, the general formalism for an effective Lagrangian was developed by Callan, Coleman, Wess, and Zumino $[119,120]$. Let us first describe the case of a chiral theory without a Higgs, which is analogous to chiral QCD with pions. In Sect. 2.2.2, we introduced the nonlinear sigma model, where the $\sigma$ particle is integrated out so the description can be made in terms of the GBs, which are contained in a dimensionless unitary matrix, $\mathbf{U}$, transforming as a bidoublet of the global chiral symmetry $S U(2)_{L} \times S U(2)_{R}$ :

$$
\mathbf{U}(x) \rightarrow L \mathbf{U}(x) R^{\dagger},
$$

where $L, R$ denote the $S U(2)_{L, R}$ global transformations.

It turns out that the Higgs sector of the SM also exhibits an accidental global chiral symmetry $S U(2)_{L} \times S U(2)_{R}$, which is spontaneously broken to the diagonal 
vector subgroup when the Higgs field acquires a vev, which actually is the so-called custodial symmetry, $S U(2)_{V}$. The symmetry can be made manifest by taking the Higgs doublet in Eq. (3.1). Then an EW bi-doublet can be written by means of both the doublet and its conjugate

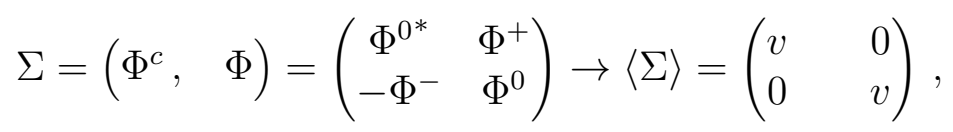

which is such that it transforms as $\Sigma \rightarrow L \Sigma R^{\dagger}$. It is easily seen from the above equation that the vev the Higgs takes upon EWSB breaks down the chiral symmetry to the (diagonal) custodial one. can be constructed in order to study Higgsless EWSB.

The custodial symmetry is actually only approximate; as the gauging of only part of the $S U(2)_{R}$ group to obtain the $U(1)_{Y}$ explicitly breaks it. Indeed, in the $g^{\prime}=0$ limit, the three massive gauge bosons $W^{ \pm}$and $Z$ have degenerate masses; while $g^{\prime} \neq 0$ leads to the well-known splitting relation

$$
M_{W}=\cos \theta_{W} M_{Z}
$$

where $\theta_{W}$ is the Weinberg angle of the SM (see Sect. 3.1). This relation must hold for any Higgs mechanism respecting the custodial symmetry and it thus provides an excellent tool to test BSM physics through the measurement of deviations to the so-called rho parameter*

$$
\rho=\frac{M_{W}^{2}}{\cos ^{2} \theta_{W} M_{Z}^{2}} .
$$

Yukawa terms in the Lagrangian also violate custodial symmetry if the masses of the two components of the fermion doublets are not degenerate.

Going back to the effective Lagrangian formulation, it is also useful to define

$$
\mathbf{V}_{\mu}(x) \equiv\left(D_{\mu} \mathbf{U}(x)\right) \mathbf{U}^{\dagger}(x)=-\mathbf{U}(x)\left(D_{\mu} \mathbf{U}^{\dagger}(x)\right),
$$

where $D_{\mu}$ is the covariant derivative

$$
D_{\mu} \mathbf{U}(x) \equiv\left(\partial_{\mu}+\frac{i g}{2} \tau^{a} W_{\mu}^{a}(x)-\frac{i g^{\prime}}{2} \tau^{3} B_{\mu}(x)\right) \mathbf{U}(x),
$$

$\tau_{a}$ are the Pauli matrices (generators of $\left.S U(2)\right), W_{\mu}^{a}, B_{\mu}$ denote the $S U(2)_{L}, U(1)_{Y}$ gauge bosons, respectively, and $g$ and $g^{\prime}$ are the corresponding gauge couplings. Under the custodial symmetry, $V_{\mu}$ transforms in the adjoint of $S U(2)_{L}$

$$
\mathbf{V}_{\mu}(x) \rightarrow L \mathbf{U}(x) L^{\dagger}
$$

while being a singlet under $S U(2)_{R}$. Another useful structure is

$$
\mathbf{T}(x) \equiv \mathbf{U}(x) \tau_{3} \mathbf{U}(x)^{\dagger}, \quad \mathbf{T}(x) \rightarrow L \mathbf{T}(x) L^{\dagger},
$$

*At tree level in the $\mathrm{SM} \rho=1$. The current experimental value for the rho parameter is $\rho=1.00040 \pm 0.00024$ [41]. Any BSM theory must respect this experimental constraint, which means there is little room for custodial violation in NP. Unless specified, the expression "custodial violating" in what follows will just refer to BSM custodial breaking effects. 
which also transforms under $S U(2)_{L}$ as a vector. However, it does not have a well-defined transformation under $S U(2)_{R}$ : it is invariant with respect to the third component (which is related to hypercharge); while it does break explicitly the other two components of the group.

With these elements it is thus possible to build the most general effective bosonic Lagrangian respecting the chiral symmetry. As is explained in Sect. 2.2, GBs are invariant under shifts, so they couple via derivatives. Each derivative in the effective operators will translate into factors of momentum in the computation of amplitudes, meaning that at sufficiently low energies (as compared to the cut-off of the effective field theory), there is an effective Lagrangian that can be organized by the number of derivatives in the different operators (which is indeed their dimensionality, since $\mathbf{U}$ is adimensional). This systematic classification of the effective chiral operators in terms of a $(p / \Lambda)$ expansion is also known as Weinberg's counting rule [121].

On the other hand, in order to include the fermions in the chiral effective basis they have to be arranged in doublets of the $S U(2)_{L}$ or $S U(2)_{R}$ symmetries. The LH doublets are those already present in the $\mathrm{SM}$ and the $\mathrm{RH}$ ones are analogous:

$$
Q_{R}=\left(\begin{array}{c}
U_{R} \\
D_{R}
\end{array}\right), \quad L_{R}=\left(\begin{array}{c}
N_{R} \\
E_{R}
\end{array}\right)
$$

where $N_{R}$ stands for RH neutrinos added to the SM (although the origin of neutrino masses will not be discussed in this thesis). The chiral (or non-linear - NL) Lagrangian containing the SM is therefore written as

$$
\mathcal{L}_{N L}=\mathcal{L}_{0}+\Delta \mathcal{L}_{N L}
$$

where the leading order $(\mathrm{LO})$ piece, $\mathcal{L}_{0}$, has the form

$$
\begin{aligned}
\mathcal{L}_{0}= & -\frac{1}{4} G_{\mu \nu} G^{\mu \nu}-\frac{1}{4} W_{\mu \nu} W^{\mu \nu}-\frac{1}{4} B_{\mu \nu} B^{\mu \nu} \\
& -\frac{v^{2}}{4} \operatorname{Tr}\left(\mathbf{V}_{\mu} \mathbf{V}^{\mu}\right)+\frac{c_{T} v^{2}}{4}\left(\operatorname{Tr}\left(\mathbf{T} \mathbf{V}_{\mu}\right)\right)^{2} \\
& +i \bar{Q} \not D Q+i \bar{L} \not D L-\frac{v}{\sqrt{2}}\left[\bar{Q}_{L} \mathbf{U} \mathcal{Y}_{Q} Q_{R}+\bar{L}_{L} \mathbf{U} \mathcal{Y}_{L} L_{R}+\text { h.c. }\right],
\end{aligned}
$$

where color, weak and flavor indices are omitted for simplicity. Also the $\theta$ term for QCD has been omitted for brevity. The Yukawa matrices are defined as $6 \times 6$ block-diagonal matrices in flavor space: $\mathcal{Y}_{Q}=\operatorname{diag}\left(Y_{U}, Y_{D}\right), \mathcal{Y}_{L}=\operatorname{diag}\left(0, Y_{E}\right)$. From Eqs. (5.12) and (5.11), it is immediate to see that the GBs kinetic term, $\operatorname{Tr}\left(\mathbf{V}_{\mu} \mathbf{V}^{\mu}\right)$, yields the masses for the EW gauge bosons.

In the bosonic sector, the next-to-leading order (NLO) is given by the AppelquistLonghitano basis, and consists in a complete basis of independent invariants made of operators with up to four derivatives. See Refs. [16-19, 122, 123].

\subsection{Including a light Higgs}

In recent years the chiral formulation has been expanded to include a light Higgs particle $h[20,21,124-127]$. We will refer to this effective expansion as the Higgs 
Effective Field Theory (HEFT). Since the physical $h$ is considered a singlet under the SM, it is customary to include its interactions in the chiral Lagrangian via adimensional generic polynomial functions [19-21]:

$$
\mathcal{F}_{i}(h)=1+2 a_{i} \frac{h}{v}+b_{i} \frac{h^{2}}{v^{2}}+\ldots
$$

As explained in Sect. 4.3, the GBs scale is $f$, so a priori the expansion should be in $h / f$ (analogously to the expansion in $\pi / f_{\pi}$ for pions in QCD), with coefficients expected to be of order one. However, it is standard to choose to write $h / v$, reflecting the fine-tuning in composite Higgs models given by $\xi=v^{2} / f^{2}$. Thus the parameters within $\mathcal{F}(h)$ are expected to be proportional to this ratio, justifying the truncated expansion of the $\mathcal{F}_{i}(h)$, up to $h^{2}$. This is the same fine-tuning that imposes a $v^{2}$ factor in front of $\operatorname{Tr}\left(\mathbf{V}_{\mu} \mathbf{V}^{\mu}\right)$ in order to obtain the right gauge boson masses, despite a $f^{2}$ would have seem more natural within the chiral expansion.

The LO Lagrangian of Eq. (5.16), is thus modified to be (disregarding the custodial breaking $\left(\operatorname{Tr}\left(\mathbf{T V}_{\mu}\right)\right)^{2}$ operator, see discussion below):

$$
\begin{aligned}
\mathcal{L}_{0}= & -\frac{1}{4} G_{\mu \nu} G^{\mu \nu}-\frac{1}{4} W_{\mu \nu} W^{\mu \nu}-\frac{1}{4} B_{\mu \nu} B^{\mu \nu}-\frac{v^{2}}{4} \operatorname{Tr}\left(\mathbf{V}_{\mu} \mathbf{V}^{\mu}\right) \mathcal{F}_{C}(h) \\
& +i \bar{Q} \not D Q+i \bar{L} \not D L-\frac{v}{\sqrt{2}}\left[\bar{Q}_{L} \mathbf{U} \mathcal{Y}_{Q}(h) Q_{R}+\bar{L}_{L} \mathbf{U} \mathcal{Y}_{L}(h) L_{R}+\text { h.c. }\right] \\
& +\frac{1}{2} \partial_{\mu} h \partial^{\mu} h-V(h)
\end{aligned}
$$

where the Yukawa coupling matrices contain also generic functions of $h$, with $Y_{I}(h)=$ $\sum_{n} Y_{I}^{(n)} \frac{h^{n}}{v^{n}}(I=U, D, E)$. This Lagrangian is equivalent to the SM one in the limit

$$
\begin{gathered}
\mathcal{F}_{C}^{S M}(h)=\left(1+\frac{h}{v}\right)^{2}, \quad \mathcal{Y}_{I}^{S M}(h)=Y_{I}\left(1+\frac{h}{v}\right) \\
V_{S M}(h)=\frac{m_{h}^{2}}{2} h^{2}+\frac{\lambda v}{2} h^{3}+\frac{\lambda}{8} h^{4}, \quad\left(m_{h}^{2}=\lambda v^{2}\right) .
\end{gathered}
$$

The reason why gauge boson kinetic terms of Eq. (5.18) do not contain any $\mathcal{F}(h)$ function is that they involve only transversal components; which are assumed not to couple with the EWSB sector at LO; but they are considered as NLO. On the other hand, the Higgs kinetic term does not contain any function $\mathcal{F}(h)$ either. The reason is that those are redundant couplings, as they can be removed via a $h$ field redefinition and then be reabsorbed in other arbitrary $h$ functions [128]. Analogously, the $\mathcal{F}(h)$ in the fermions kinetic term can be absorbed through a redefinition of the fermion fields and then be absorbed in the Yukawa generic $h$ functions, see Appendix B in Ref. [127].

The NLO Lagrangian contains a non-redundant basis of operators that is constructed with the same techniques as the Appelquist-Longhitano basis mentioned previously; but allowing also for the $\mathcal{F}_{i}(h)$ structures, together with all its possible derivatives. See the references mentioned at the beginning of the section. 
Finally, it is interesting to mention that the ordering of the non-linear tower of operators is a non-trivial issue, since the chiral Lagrangian is in fact mixing two expansions very different in nature:

- On the one hand, the chiral expansion given by the number of derivatives for the would-be GBs inside $\mathbf{U}$, as explained above. The terms at LO in this case have mass dimension two (see below a comment on the case of the custodial-breaking operator of dimension two).

- On the other hand, there is a SM-like weakly-interacting expansion for the fermions and the transversal components of the gauge bosons, where the interactions are organized through the canonical dimensions of the operators, with the kinetic terms, of mass dimensions equal four, included at LO.

Naturally, when there are interactions between the two sectors, the analysis becomes tricky. The first attempt to propose a unified counting rule was made some time ago with the so-called Naive Dimensional Analysis (NDA) [100]. See also Refs. [129,130] for related work. Besides, very recently, different works on the issue have appeared in the literature [131-135], reviving the discussion.

In other words, the SMEFT has a clear LO versus NLO, as the LO is the SM and then the tower of effective operators is well defined. In contrast, there is a certain freedom in the choice of the precise terms to be included in the LO Lagrangian for non-linear realizations of EWSB. Furthermore, in the literature other supplementary choices are made. For instance, the term with

$$
\left(\operatorname{Tr}\left(\mathbf{T} \mathbf{V}_{\mu}\right)\right)^{2}
$$

of mass dimensions two, is often considered phenomenologically as NLO and we will also do so in the works described in this thesis. The reason is that it breaks the accidental custodial symmetry in the SM, while experimentally these effects are very constrained. In particular, it contributes to the mass term of the $Z$ boson, but not to the $W$ mass. This translates into a sizable contribution to the $\rho$ parameter in Eq. (5.9) that imposes a constraint of order $10^{-2}$ on the coefficient $c_{T}$.

\subsection{Linear vs. Non-linear approach}

Besides the chiral effective Lagrangian, there is the Standard Model Effective Field Theory (SMEFT); which is the effective theory built with the SM fields and symmetries and is often referred to as the linear Lagrangian. The tower of operators is organized by their canonical mass dimension and the LO contains the renormalizable interactions of $d=4$ and coincides with the SM Lagrangian:

$$
\mathcal{L}_{\text {linear }}=\mathcal{L}_{S M}+\Delta \mathcal{L}_{d>4},
$$

Linear effective Lagrangians are useful to describe perturbative BSM physics. The first complete classification of $d=6$ linear invariants was done in Ref. [136]; while a 
more recent work [137] corrected some redundancies and proposed the basis of operators that nowadays is widely used. An alternative, also common, choice, more suitable for phenomenology in the EW boson sector, is the Hagiwara-Ishihara-SzalapskiZeppenfeld (HISZ) basis [138, 139]. The main differences between the linear and non-linear expansions are listed in the following paragraphs.

In the linear expansion the Higgs field is not a generic singlet; but is embedded in the SM doublet $\Phi$ of Eq. (3.1), together with the three GBs of the SM EWSB. $\Phi$ transforms linearly under the SM gauge group; hence the name linear. As a result of this linear behavior, insertions of the physical Higgs, $h$ always come accompanied of the GBs. Furthermore, they always appear in structures of the form $(v+h)^{n}$; whereas in the chiral expansion the Higgs couplings are completely free.

As stated above, the SMEFT is organized as an expansion in canonical mass dimensions. The expansion in the chiral Lagrangian is more complicated though. The GBs sector follows an expansion in derivatives and are contained in the matrix $\mathbf{U}$, which is an independent object from the physical $h$. Besides, $\mathbf{U}$ has no dimensions, which causes a reshuffling of the interactions at each order, as compared to the linear tower. In particular, as a consequence of the non-linear behavior, insertions of the longitudinal gauge boson components (GBs) in the chiral Lagrangian are less suppressed than in the SMEFT.

As a result of the above features, the non-linear Lagrangian contains a larger number of invariant structures at any given order, as compared to the SMEFT. This can be seen by replacing

$$
\Phi \rightarrow \frac{(v+h)}{\sqrt{2}} \mathbf{U}\left(\begin{array}{l}
0 \\
1
\end{array}\right)
$$

inside the linear operators, it is possible to identify the corresponding non-linear invariants. For example, taking the following operator of the HISZ basis:

$$
\begin{aligned}
\mathcal{O}_{B}= & \frac{i g^{\prime}}{2} D_{\mu} \Phi^{\dagger} B^{\mu \nu} D_{\nu} \Phi \\
& \longrightarrow \quad \frac{i g^{\prime}}{4} B^{\mu \nu}\left(\frac{(v+h)^{2}}{2} \operatorname{Tr}\left(\mathbf{T}\left[\mathbf{V}_{\mu}, \mathbf{V}_{\nu}\right]\right)+\partial_{\mu}\left((v+h)^{2}\right) \operatorname{Tr}\left(\mathbf{T V}_{\mu}\right)\right)
\end{aligned}
$$

As a consequence, effects that are expected to be correlated in the linear Lagrangian appear to be uncorrelated, in general, in the non-linear series. On the other hand, in other cases, effects expected to appear at a certain order in the non-linear expansion are found to appear as higher-order corrections, i.e. they are more suppressed, in the linear series (see for example Sect. 11.2). Ref. [140] contains a detailed study on how to disentangle both scenarios experimentally; since this fact might translate into different predictions to be tested at accelerators such as the LHC.

In short, in the case of the Higgs particle being a pGB, the best approach is a general non-linear effective Lagrangian with a light $h$ particle, which ultimately needs to be supplemented by model-dependent relations. Also, if the $h$ field has a component that is a generic SM singlet, or it is not embedded in the SM doublet representation, the appropriate tool continues to be a non-linear effective Lagrangian with arbitrary coefficients. These can actually describe the corresponding linear interactions by imposing on them the appropriate constraints in order to recover the Higgs doublet 
structure. Therefore, in order to study the EWSB in all generality, the non-linear approach is the object of study of the most part of this thesis, as is further described in the following chapters. 


\section{Chapter 6}

\section{Renormalization of the scalar sector}

\subsection{Renormalization in EFTs}

EFTs are generally not renormalizable strictly speaking, in the sense that there is not a finite set of operators to absorb all the infinities arising by quantum corrections, as in the SM. However, this is not really a problem; it only means that it will be valid just until the scale of its UV completion. In the case of the chiral expansion there is actually a general, systematic and consistent procedure to renormalize it, called Chiral Perturbation Theory, which has already been used extensively and successfully in the study of QCD at low energies; see, for instance, Ref. [141]. As it will be done below, the method consists in taking first up to two-derivatives as the LO Lagrangian, $\mathcal{L}_{0}+\mathcal{L}_{2}$; which is used both at tree and one-loop levels. Then $\mathcal{L}_{4}$ is taken as the NLO, so it is used only at tree level, as it will yield contributions of the same order as one-loop $\mathcal{L}_{2}$. As a consequence, to say that the effective theory can be renormalized means that all one-loop divergences generated by $\mathcal{L}_{2}$ have to be absorbed by the terms in $\mathcal{L}_{4}$ and nothing else. Therefore, the procedure ensures that there is a finite set of parameters to absorb the divergences, order by order in the chiral expansion.

In this chapter we explore the one-loop renormalization to the scalar sector of the HEFT Lagrangian [142]; i.e. we will just consider operators containing scalar fields: the $h$ particle plus the Goldstone bosons. This analysis is not irrelevant, since at high energies $\left(E>>m_{V}\right)$ the GBs correspond to the longitudinal components of the massive gauge bosons, as is dictated by the so-called Equivalence Theorem [143-145]. Basically, it states that "the amplitude for emission or absorption of a longitudinally polarized gauge boson $\left(V_{L}\right)$ is equal, up to corrections of order $\mathcal{O}\left(m_{V} / \sqrt{s}\right)$, to the amplitude for emission or absorption of the associated Goldstone boson"; where $m_{V}$ is the gauge boson mass and $s$ is centre-of-mass energy. In other words, the amplitude $\mathcal{M}$ for an scattering involving longitudinally polarized gauge bosons, $V_{L}$, can be expressed in terms of the amplitude involving the associated GBs, $\pi$, by the 
equation:

$$
\begin{aligned}
& \mathcal{M}\left(V_{L}\left(p_{1}\right), \ldots, V_{L}\left(p_{n}\right)+X \rightarrow V_{L}\left(q_{1}\right), \ldots, V_{L}\left(q_{m}\right)+Y\right) \\
& =(-i)^{n+m} \mathcal{M}\left(\pi\left(p_{1}\right), \ldots, \pi\left(p_{n}\right)+X \rightarrow \pi\left(q_{1}\right), \ldots, \pi\left(q_{m}\right)+Y\right)\left(1+\mathcal{O}\left(m_{V} / \sqrt{s}\right)\right) .
\end{aligned}
$$

The one-loop renormalization has been extensively studied in the literature for the linear effective Lagrangian (see for instance [8-15]), and up to some extent for the non-linear expansion [16,22-27]. We also perform a one-loop study; but since we are more interested in analyzing the operators structure of the effective Lagrangian, we take an off-shell approach; in contrast to recent work that focused on phenomenology from on-shell scatterings.

Performing the renormalization off-shell guarantees that all counterterms required for consistency are identified, as each Green function is renormalized independently (see Sect. 6.3). In this way, this makes it possible to see if the basis of chiral invariant scalar operators is complete. On the other hand, we are also interested in obtaining the most general full set of counterterms in the scalar sector of the effective Lagrangian; without disregarding operators that are usually traded by fermionic ones via the equations of motion. Only when considering all possible couplings and all SM fields, should one choose which set of operators to keep in order to avoid redundancies.

Finally, doing the renormalization off-shell was also instructive because it revealed the existence of apparent chiral non-invariant divergences (see Sect. 6.3 and more in particular, the subsection 6.3.6); which were already encountered in the off-shell renormalization of the non-linear sigma model [146]. There, non-invariant counterterms were seen to be required to absorb the divergences from the off-shell renormalization of the pion four-point function and at the same time were shown to have no physical implication, thus proving that the chiral invariance is actually preserved. In our work, we also found new divergences of this type involving the light $h$ particle. Moreover, we also explored a general parametrization for the GB matrix, and the offshell renormalization provided a nice check that the freedom in the parametrization had no physical consequence.

\subsection{The scalar Lagrangian}

The formalism adopted here to describe in all generality a light dynamical scalar boson, denoted by $h$, in the context of a generic non-linear realization of EWSB is based on Refs. [21,30,124,125,140]. As explained in Sect. 5.3, this consists of a chiral effective Lagrangian including a light $h$ as a SM singlet, such that in general its couplings are different to those of a Higgs embedded as a SM doublet.

Since the focus is on the physics of the sector including the three low-energy GBs, $\pi_{a}$, and the Higgs scalar, $h$; fermions will not be considered and the gauge couplings $g$ and $g^{\prime}$ are switched off. Furthermore, we also omit custodial breaking operators, 
as phenomenology indicates that the size of their coefficients must be strongly constrained (see footnote in page 53). In consequence, and as no gauge nor Yukawa interactions are considered here, no custodial-breaking counterterm will be required by the renormalization procedure to be present among the four-derivative operators in $\mathcal{L}_{4}$, as we will see in Sect. 6.3.

The non-linear Lagrangian is organized as

$$
\mathcal{L}=\mathcal{L}_{0}+\mathcal{L}_{2}+\mathcal{L}_{4}
$$

where the $\mathcal{L}_{i}$ subindex indicates the number of derivatives; as it was explained in Sect. 5.2:

$$
\begin{aligned}
\mathcal{L}_{0} & =-V(h)=-\mu_{1}^{3} h-\frac{1}{2} m_{h}^{2} h^{2}-\frac{\mu_{3}}{3 !} h^{3}-\frac{\lambda}{4 !} h^{4}, \\
\mathcal{L}_{2} & =\frac{1}{2}\left(\partial_{\mu} h \partial^{\mu} h\right) \mathcal{F}_{H}+\frac{v^{2}}{4} \mathcal{F}_{C} \operatorname{Tr}\left(D_{\mu} \mathbf{U} D^{\mu} \mathbf{U}^{\dagger}\right) \\
& =\frac{1}{2}\left(1+2 a_{H} \frac{h}{v}+b_{H} \frac{h^{2}}{v^{2}}\right)\left(\partial_{\mu} h \partial^{\mu} h\right)+\frac{v^{2}}{4}\left(1+2 a_{C} \frac{h}{v}+b_{C} \frac{h^{2}}{v^{2}}\right) \operatorname{Tr}\left(D_{\mu} \mathbf{U} D^{\mu} \mathbf{U}^{\dagger}\right), \\
\mathcal{L}_{4} & =c_{i} \mathcal{P}_{i} .
\end{aligned}
$$

$\mathcal{L}_{0}$ contains a general Higgs potential, for which only up to terms quartic in $\mathrm{h}$ are made explicit, and arbitrary coefficients $\mu_{i}$ and $\lambda$. It will be assumed that the $h$ field is the physical one, with $\langle h\rangle=0$, so the linear term in $h$ in the Lagrangian is just kept in order to cancel the tadpole amplitude at one loop; see Sect. 6.3.1.

$\mathcal{L}_{2}$ contains a kinetic term for the Higgs and another for the GBs, contained inside the matrix $\mathbf{U}$ (see Sect. 5.2). We analyze in the next section the freedom in defining this matrix and work with a general parametrization truncated up to some order in $\pi / v$. Both kinetic terms appear with some arbitrary functions, $\mathcal{F}_{H, C}(h)$, attached, which represent the chiral-symmetry breaking interactions of h. In the SM limit only $a_{C}$ and $b_{C}$ survive, with $a_{C}=b_{C}=1$. As already commented in the introduction to chiral Lagrangians, the $v^{2}$ in front of the kinetic term for the GBs is fine-tuned in order to obtain the right mass for the EW gauge bosons.

The computation of Ref. [142], which is summarized in this chapter was performed keeping an explicit $\mathcal{F}_{H}(h)$ and thus we will keep it here. However, it is possible to absorb the coefficients in $\mathcal{F}_{H}(h)$ inside the free parameters within other generic functions by making a non-linear redefinition of $h$; as it is shown in Ref. [128]. Making $a_{H}=b_{H}=0$ in the results displayed in the next sections does not change the main conclusions on the counterterms that are needed to cancel the divergences at one-loop; since it can be checked that all the counterterm coefficients in $\mathcal{L}_{4}$ will be induced for generic $a_{C}$ and $b_{C}$; even if $\mathcal{F}_{H}(h)=1$. Indeed, the only contributions that will vanish completely would be those to $\delta a_{H}$ and $\delta b_{H}$.

Finally, $\mathcal{L}_{4}$ contains the NLO operators, with arbitrary constant coefficients, $c_{i}$, which are listed in Table 6.1, where $\square \equiv \partial_{\mu} \partial^{\mu}, V_{\mu} \equiv\left(D_{\mu} U\right) U^{\dagger}$ and the covariant derivative is simply the partial derivative $D_{\mu}=\partial_{\mu}$, since the transverse gauge field components are not considered in the analysis. The notation differs slightly from 
that used in Ref. [140]. For simplicity, redundant parameters have been eliminated via the following substitutions:

$$
\begin{aligned}
& \partial_{\mu} \mathcal{F}_{i}(h) \rightarrow \partial_{\mu} h \mathcal{F}_{i}(h), \\
& \partial_{\mu} \mathcal{F}_{i}(h) \partial_{\nu} \tilde{\mathcal{F}}_{i}(h) \rightarrow \partial_{\mu} h \partial_{\nu} h \mathcal{F}_{i}(h), \\
& \square \mathcal{F}_{i}(h) \rightarrow \square h \mathcal{F}_{i}(h), \\
& c_{i} \mathcal{F}_{i}(h) \equiv c_{i}+2 a_{i} h / v+b_{i} h^{2} / v^{2}
\end{aligned}
$$

\section{Parametrization of the GB matrix}

The non-linear $\sigma$ model, which is equivalent to our Lagrangian without the $h$ particle, can be written in terms of a derivative "covariant" under the non-linear chiral symmetry, $D_{\mu}[147]$ :

$$
\mathcal{L}_{N L}=\frac{v^{2}}{4} \operatorname{Tr}\left(D_{\mu} \mathbf{U} D^{\mu} \mathbf{U}^{\dagger}\right)=D_{\mu} \boldsymbol{\pi} D^{\mu} \boldsymbol{\pi}=G_{i j}\left(\boldsymbol{\pi}^{2}\right) \partial_{\mu} \pi_{i} \partial^{\mu} \pi_{j}
$$

where $G_{i j}\left(\boldsymbol{\pi}^{2}\right)$ can be interpreted as the metric of a 3-sphere in which the GBs, $\boldsymbol{\pi}=\left(\pi_{1}, \pi_{2}, \pi_{3}\right)$, live, and the freedom of parametrization simply translates into a coordinate transformation (see Ref. [146] and references therein, and also more recent work [148-150]*). In [147], it is shown that different linear realizations of the chiral symmetry lead to different metrics, which correspond to different parametrizations of the GB matrix; are all connected via redefinitions of the GB fields. All these transformations are equivalent with respect to the dynamics of the pion fields, since the non-linear transformation induced on them is unique, and as a consequence on-shell quantities will be independent of the choice of parametrization for the $\mathbf{U}$ matrix, as will be shown below.

To show the different possibilities for the $\mathbf{U}$ matrix explicitly here; let us parametrize the GB matrix through two general functions, $\mathrm{X}$ and $\mathrm{Y}:^{\dagger}$

$$
\mathbf{U} \equiv X(z)+i \frac{\boldsymbol{\tau} \cdot \boldsymbol{\pi}}{v} Y(z)
$$

where $\boldsymbol{\tau}$ denotes the Pauli matrices and $z \equiv \pi^{2} / v^{2} . \quad Y(0)= \pm 1$ is required for canonically normalized GB kinetic terms. $X(z)$ and $Y(z)$ are related via the unitarity condition $\mathbf{U U}^{\dagger}=\mathbf{U}^{\dagger} \mathbf{U}=\mathbf{1}$. Therefore

$$
\mathbf{U}=\sqrt{1-z Y(z)^{2}}+i \frac{\boldsymbol{\tau} \cdot \boldsymbol{\pi}}{v} Y(z)
$$

The Lagrangian is thus invariant under the transformation $Y \rightarrow-Y$, or equivalently, $\boldsymbol{\pi} \rightarrow-\boldsymbol{\pi}$ (only operators with even powers of the $\boldsymbol{\pi}$ fields will appear).

*Refs. [148-150] provide a geometric formulation of the scalar sector of Higgs EFT. In this way compute different experimental observables in terms of geometric invariants such as the curvature of the scalar field manifold; thus finding a way to disentangle through experiment between a scalar sector transforming linearly (flat manifold) or non-linearly (curved manifold) under the EW gauge group.

†The function $f(x)$ of Ref. [147] corresponds to $X(z) / Y(z)$ here. 


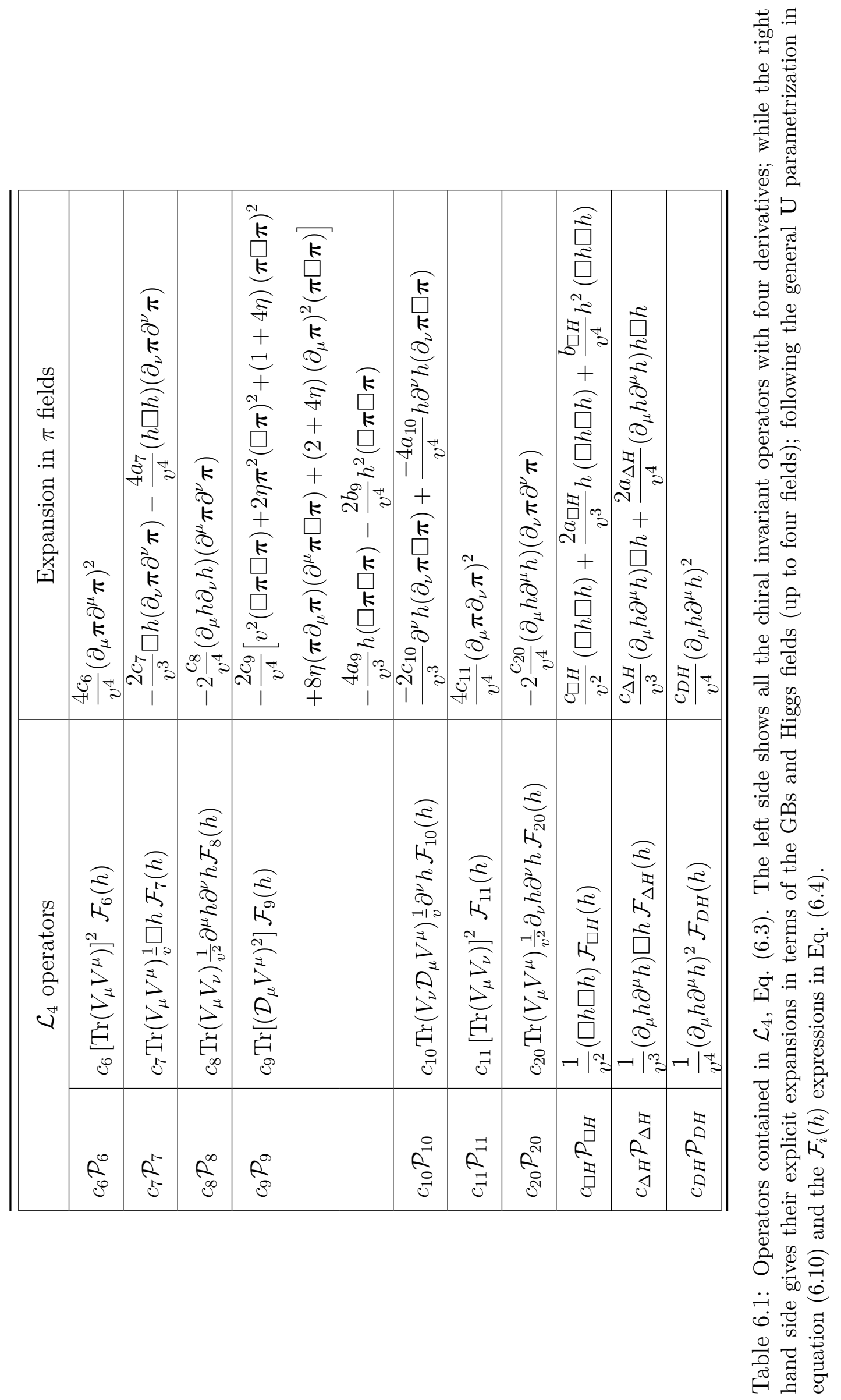


In terms of those functions, the $G_{i j}$ metric is

$$
G_{i j}\left(\boldsymbol{\pi}^{2}\right)=Y(z)^{2} \delta_{i j}+4\left(X^{\prime}(z)^{2}+z Y^{\prime}(z)^{2}+Y(z) Y^{\prime}(z)\right) \frac{\pi_{i} \pi_{j}}{v^{2}},
$$

where the primes indicate derivatives with respect to the $\mathrm{z}$ variable.

An arbitrary parameter, $\eta$, will encode the parametrization dependence. It is defined in the expansion of the $\mathrm{Y}$ function:

$$
Y(z)=1+\eta z+O\left(z^{2}\right)
$$

We truncate the expression at the quadratic order, as our analysis will only require to consider terms up to four GB fields in $\mathcal{L}_{2}$. One could think of terms up to six pion fields, though, which would contribute to 4-point functions by joining two of the GB legs into a closed loop. However, as the GBs are massless, this will result in a vanishing contribution. As a consequence, in practice it will suffice to consider inside $\mathbf{U}$ terms only up to cubic powers on the $\boldsymbol{\pi}$ fields:

$$
\mathbf{U}=1-\frac{\boldsymbol{\pi}^{2}}{2 v^{2}}-\left(\eta+\frac{1}{8}\right) \frac{\boldsymbol{\pi}^{4}}{v^{4}}+\frac{i(\boldsymbol{\pi} \boldsymbol{\tau})}{v}\left(1+\eta \frac{\boldsymbol{\pi}^{2}}{v^{2}}\right)+\ldots
$$

Specific values of $\eta$ correspond to different parametrizations, up to terms with four GBs. For instance, for the two most popular parametrizations:

- $\eta=0$ yields the square root parametrization: $\mathbf{U}=\sqrt{1-\boldsymbol{\pi}^{2} / v^{2}}+i(\boldsymbol{\pi} \cdot \boldsymbol{\tau}) / v$.

- $\eta=-1 / 6$ yields the exponential parametrization: $\mathbf{U}=\exp (i \boldsymbol{\pi} \cdot \boldsymbol{\tau} / v)$.

In terms of the GB fields with the parametrization freedom described in this section, the $\mathcal{L}_{2}$ Lagrangian results to be

$$
\begin{aligned}
\mathcal{L}_{2} & =\frac{1}{2} \partial_{\mu} h \partial^{\mu} h\left(1+2 a_{H} \frac{h}{v}+b_{H} \frac{h^{2}}{v^{2}}\right) \\
& +\left\{\frac{1}{2} \partial_{\mu} \boldsymbol{\pi} \partial^{\mu} \boldsymbol{\pi}+\frac{\left(\boldsymbol{\pi} \partial_{\mu} \boldsymbol{\pi}\right)^{2}}{2 v^{2}}+\eta\left[\frac{\boldsymbol{\pi}^{2}\left(\partial_{\mu} \boldsymbol{\pi}\right)^{2}}{v^{2}}+2 \frac{\left(\boldsymbol{\pi} \partial_{\mu} \boldsymbol{\pi}\right)^{2}}{v^{2}}\right]\right\}\left(1+2 a_{C} \frac{h}{v}+b_{C} \frac{h^{2}}{v^{2}}\right),
\end{aligned}
$$

where terms containing more than four fields are to be disregarded in the computation. In Table 6.1 the expressions up to four fields of the chiral invariant operators with four derivatives are shown. It will be shown in the renormalization procedure below that all of them need be present in $\mathcal{L}_{4}$ as counterterms.

\section{Counterterm Lagrangian}

The counterterm Lagrangian is obtained, as usual, by writing the bare parameters and fields in $\mathcal{L}_{0}, \mathcal{L}_{2}$ and $\mathcal{L}_{4}$ in terms of the renormalized ones. The bare (b) quantities can be written in terms of renormalized quantities (we choose not to write a superindex $r$ for them for simplicity) as follows:

$$
\begin{array}{ll}
h_{b}=\sqrt{Z_{h}} h, & \delta_{h} \equiv Z_{h}-1, \\
\boldsymbol{\pi}_{b}=\sqrt{Z_{\pi}} \boldsymbol{\pi}, & \delta_{\pi} \equiv Z_{\pi}-1,
\end{array}
$$




$$
\begin{array}{ll}
v_{b}^{2}=Z_{\pi}\left(v^{2}+\delta v^{2}\right) \mu^{-\varepsilon}, & a_{C}^{b}=\frac{1}{Z_{\pi}^{1 / 2} Z_{h}^{1 / 2}}\left(a_{C}+\delta a_{C}+\frac{a_{C}}{2} \frac{\delta v^{2}}{v^{2}}\right), \\
\left(m_{h}^{2}\right)^{b}=\frac{1}{Z_{h}}\left(m_{h}^{2}+\delta m_{h}^{2}\right), & b_{C}^{b}=\frac{1}{Z_{h}}\left(b_{C}+\delta b_{C}+b_{C} \frac{\delta v^{2}}{v^{2}}\right), \\
\left(\mu_{1}^{3}\right)^{b}=\frac{1}{Z_{h}^{1 / 2}}\left(\mu_{1}^{3}+\delta \mu_{1}^{3}\right) \mu^{3 \varepsilon / 2}, & a_{H}^{b}=\frac{Z_{\pi}^{1 / 2}}{Z_{h}^{3 / 2}}\left(a_{H}+\delta a_{H}+\frac{a_{H}}{2} \frac{\delta v^{2}}{v^{2}}\right), \\
\mu_{3}^{b}=\frac{1}{Z_{h}^{3 / 2}}\left(\mu_{3}+\delta \mu_{3}\right) \mu^{\varepsilon / 2}, & b_{H}^{b}=\frac{Z_{\pi}}{Z_{h}^{2}}\left(b_{H}+\delta b_{H}+b_{H} \frac{\delta v^{2}}{v^{2}}\right) .
\end{array}
$$

Dimensional regularization is used as regularization scheme. This avoids quadratic divergences appearing explicitly, which could lead to further technical complications $[151,152]$. In these expressions $\mu$ is the renormalization scale and is introduced in the Lagrangian, so the renormalized parameter has the same dimensions as the bare one, when doing dimensional regularization, where we will move from number of dimensions $d=4$ to $d=4-\varepsilon$.

From these redefinitions, the counterterm Lagrangian arising from the leading order $\mathcal{L}_{0}$ and $\mathcal{L}_{2}$ reads:

$$
\begin{aligned}
\delta \mathcal{L}_{0}+\delta \mathcal{L}_{2} & =\frac{1}{2} \partial_{\mu} h \partial^{\mu} h\left(\delta_{h}+2 \delta a_{H} \frac{h}{v}+\delta b_{H} \frac{h^{2}}{v^{2}}\right)-\frac{1}{2} \delta m_{h}^{2} h^{2}-\delta \mu_{1}^{3} h-\frac{\delta \mu_{3}}{3 !} h^{3}-\frac{\delta \lambda}{4 !} h^{4} \\
& +\frac{1}{2} \partial_{\mu} \boldsymbol{\pi} \partial^{\mu} \boldsymbol{\pi}\left(\delta_{\pi}+2 \delta a_{C} \frac{h}{v}+\delta b_{C} \frac{h^{2}}{v^{2}}\right) \\
& +\left(\delta_{\pi}-\frac{\delta v^{2}}{v^{2}}\right) \frac{1}{2 v^{2}}\left(\left(\boldsymbol{\pi} \partial_{\mu} \boldsymbol{\pi}\right)\left(\boldsymbol{\pi} \partial^{\mu} \boldsymbol{\pi}\right)+2 \eta\left(\boldsymbol{\pi}^{2}\left(\partial_{\mu} \boldsymbol{\pi}\right)^{2}+2\left(\boldsymbol{\pi} \partial_{\mu} \boldsymbol{\pi}\right)^{2}\right)\right) .
\end{aligned}
$$

For the coefficients in the NLO Lagrangian we have:

$$
\begin{array}{rlr}
X_{i}^{b} & =\left(X_{i}+\delta X_{i}+2 X_{i} \frac{\delta v^{2}}{v^{2}}\right) \mu^{-\epsilon}, & X_{i}=c_{6}, c_{9}, c_{11}, \\
X_{i}^{b}=\frac{Z_{\pi}^{1 / 2}}{Z_{h}^{1 / 2}}\left(X_{i}+\delta X_{i}+\frac{3}{2} X_{i} \frac{\delta v^{2}}{v^{2}}\right) \mu^{-\epsilon}, & X_{i}=c_{7}, a_{9}, c_{10}, \\
X_{i}^{b}=\frac{Z_{\pi}}{Z_{h}}\left(X_{i}+\delta X_{i}+2 X_{i} \frac{\delta v^{2}}{v^{2}}\right) \mu^{-\epsilon}, & X_{i}=a_{7}, c_{8}, b_{9}, a_{10}, c_{20}, \\
X_{i}^{b}=\frac{Z_{\pi}}{Z_{h}}\left(X_{i}+\delta X_{i}+X_{i} \frac{\delta v^{2}}{v^{2}}\right) \mu^{-\epsilon}, & X_{i}=c_{\square H}, \\
X_{i}^{b}=\frac{Z_{\pi}^{3 / 2}}{Z_{h}^{3 / 2}}\left(X_{i}+\delta X_{i}+\frac{3}{2} X_{i} \frac{\delta v^{2}}{v^{2}}\right) \mu^{-\epsilon}, & X_{i}=a_{\square H}, c_{\Delta H}, \\
X_{i}^{b}=\frac{Z_{\pi}^{2}}{Z_{h}^{2}}\left(X_{i}+\delta X_{i}+2 X_{i} \frac{\delta v^{2}}{v^{2}}\right) \mu^{-\epsilon}, & X_{i}=b_{\square H}, a_{\Delta H}, c_{D H} .
\end{array}
$$

And $\delta \mathcal{L}_{4}$ is simply given by $\mathcal{L}_{4}$ with the substitution $c_{i}, a_{i}, b_{i} \rightarrow \delta c_{i}, \delta a_{i}, \delta b_{i}$, except 
for the operator $\mathcal{P}_{9}$; which also involves $\delta v^{2}$ :

$$
\begin{aligned}
\delta\left(c_{9} \mathcal{P}_{9}\right)= & -\frac{2 \delta c_{9}}{v^{4}}\left((1+4 \eta)(\boldsymbol{\pi} \square \boldsymbol{\pi})^{2}+2(1+2 \eta)\left(\partial_{\mu} \boldsymbol{\pi}\right)^{2}(\boldsymbol{\pi} \square \boldsymbol{\pi})+2 \eta \boldsymbol{\pi}^{2}(\square \boldsymbol{\pi})^{2}\right. \\
& \left.+8 \eta\left(\boldsymbol{\pi} \partial_{\mu} \boldsymbol{\pi}\right)\left(\partial^{\mu} \boldsymbol{\pi} \square \boldsymbol{\pi}\right)\right)-\frac{4 \delta a_{9}}{v^{3}} h(\square \boldsymbol{\pi} \square \boldsymbol{\pi})-\frac{2 \delta b_{9}}{v^{4}} h^{2}(\square \boldsymbol{\pi} \square \boldsymbol{\pi}) \\
& -\frac{2}{v^{2}}\left(\delta c_{9}-\frac{\delta v^{2}}{v^{2}}\right)(\square \boldsymbol{\pi} \square \boldsymbol{\pi}) .
\end{aligned}
$$

Among the Lagrangian parameters above, $v$ plays the special role of being the characteristic scale of the Goldstone bosons (that is, of the longitudinal degrees of freedom of the electroweak bosons), analogous to the pion decay constant in QCD. It turns out that the counterterm coefficient $\delta v^{2}=0$ as shown below. We have left explicit the $\delta v^{2}$ dependence all through the paper, though, in case it may be interesting to apply our results to some scenario which includes sources of explicit chiral symmetry breaking in a context different than the SM one; it also serves as a check-point of our computations.

\subsection{Renormalization of the off-shell Green func- tions}

In this section the results for the renormalization of the 1-2-, 3-, and 4-point functions involving $h$ and/or $\boldsymbol{\pi}$ are presented in a general $\mathbf{U}$ parametrization; which is specified by the $\eta$ parameter in equation (6.10).

As renormalization procedure we choose minimal subtraction scheme (MS); which means that we make the coefficients of the counterterms be just equal to the infinite terms arising when performing the 1-loop computation. The divergence is denoted by

$$
\Delta_{\varepsilon}=+\frac{1}{(4 \pi)^{2}} \frac{2}{\varepsilon}
$$

To follow the modified Minimal Subtraction scheme $(\overline{M S})$ would essentially be the same, but $\Delta_{\varepsilon}$ would stand for $\frac{1}{(4 \pi)^{2}}\left(\frac{2}{\varepsilon}-\gamma_{E}+\log (4 \pi)\right)$, with $\gamma_{E}$ the EulerMascheroni constant. FeynRules, FeynArts, and FormCalc [153-157] have been employed to compute the one-loop amplitudes.

An overview of the results that we are going to detail in the following subsections, is shown in Table 6.2, with the operator coefficients contributing to amplitudes involving GBs and/or $h$, up to 4-point vertices. It also serves to illustrate the main conclusion from the computation: all operators in $\mathcal{L}_{4}$ will be shown to be required by the renormalization procedure. Furthermore we have checked that they are all independent, and they can be thus chosen as a non-redundant scalar set to be embedded in a complete Lagrangian (see below further discussion on the redundancy of the complete basis). 


\begin{tabular}{|c|c|c|c|c|c|c|c|}
\hline & \multicolumn{7}{|c|}{ Amplitudes } \\
\hline & $2 h$ & $3 h$ & $4 h$ & $2 \pi$ & $2 \pi h$ & $2 \pi 2 h$ & $4 \pi$ \\
\hline $\mathcal{P}_{6}$ & & & & & & & $c_{6}$ \\
\hline $\mathcal{P}_{7}$ & & & & & $c_{7}$ & $a_{7}$ & \\
\hline $\mathcal{P}_{8}$ & & & & & & $c_{8}$ & \\
\hline $\mathcal{P}_{9}$ & & & & $c_{9}$ & $a_{9}$ & $b_{9}$ & $c_{9}$ \\
\hline $\mathcal{P}_{10}$ & & & & & $c_{10}$ & $a_{10}$ & \\
\hline $\mathcal{P}_{11}$ & & & & & & & $c_{11}$ \\
\hline $\mathcal{P}_{20}$ & & & & & & $c_{20}$ & \\
\hline $\mathcal{P}_{\square H}$ & $c_{\square H}$ & $a_{\square H}$ & $b_{\square H}$ & & & & \\
\hline $\mathcal{P}_{\Delta H}$ & & $c_{\Delta H}$ & $a_{\Delta H}$ & & & & \\
\hline $\mathcal{P}_{D H}$ & & & $c_{D H}$ & & & & \\
\hline
\end{tabular}

Table 6.2: This table summarizes the operators in $\mathcal{L}_{4}$ (see equation (6.3) and Table 6.1) contributing to 2-, 3-, and 4-point amplitudes involving GBs and/or $h$ fields. The specific operator coefficients contributing to each amplitude are indicated, following the $c_{i} \mathcal{F}_{i}$ expansion in Eq. (6.4).

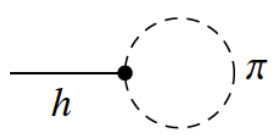

1

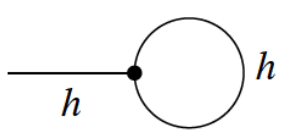

2

Figure 6.1: Diagram contributing to the Higgs 1-point function.

\section{1-point functions}

Because of chiral symmetry GBs always come in even numbers in any vertex, unlike Higgs particles, thus tadpole contributions may be generated only for the latter. At tree-level it would suffice to set $\mu_{1}=0$ in $V(h)$ (see Eq. (6.3)) in order to ensure $\langle h\rangle=0$. At one-loop, a tadpole term is induced from the triple Higgs couplings $\mu_{3}$ and $a_{H}$, though, via the Feynman diagram in Fig. 6.1. We simply use the tadpole term to get rid of this contribution in the renormalized Lagrangian by choosing $\mu_{1}^{r}=0$, so the counterterm required to absorb the divergency takes the form

$$
\delta \mu_{1}^{3}=m_{h}^{2}\left(\frac{\mu_{3}}{2}-a_{H} \frac{m_{h}^{2}}{v}\right) \Delta_{\varepsilon},
$$

and has no further impact on the rest of the Lagrangian.

\section{2-point functions}

The diagrams contributing to the GB self-energy are shown in Fig. 6.2. The di- 


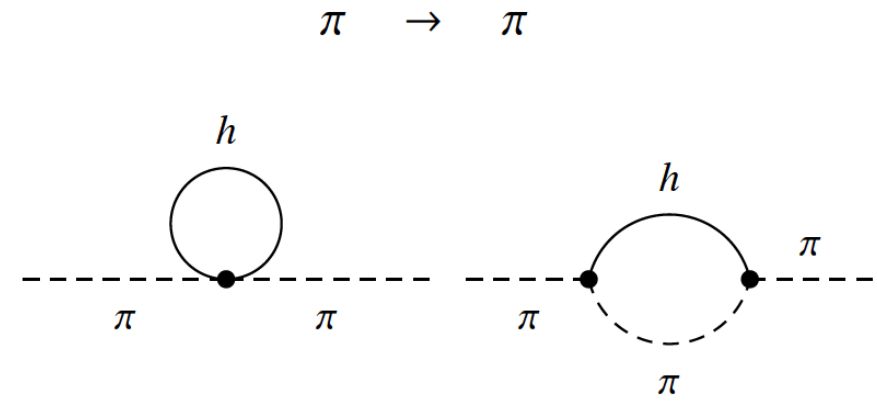

Figure 6.2: Diagrams contributing to the $\pi$ self-energy.

vergent part of the amplitudes, $\Pi_{\text {div }}^{i j}\left(p^{2}\right) \Delta_{\varepsilon}$, and the counterterm structure, from Eq. (6.14), are given by

$$
\begin{aligned}
& \Pi_{\text {div }}^{i j}\left(p^{2}\right)=\left[p^{2}\left(a_{C}^{2}-b_{C}\right) \frac{m_{h}^{2}}{v^{2}}+p^{4} \frac{a_{C}^{2}}{v^{2}}\right] \delta_{i j}, \\
& \Pi_{c t r}^{i j}\left(p^{2}\right)=\left[p^{2} \delta_{\pi}-p^{4} \frac{4}{v^{2}}\left(c_{9}-\frac{\delta v^{2}}{v^{2}}\right)\right] \delta_{i j} .
\end{aligned}
$$

Matching the momenta structures, the expressions are found for the counterterm coefficients:

$$
\begin{aligned}
\delta_{\pi} & =-\left(a_{C}^{2}-b_{C}\right) \frac{m_{h}^{2}}{v^{2}} \Delta_{\varepsilon}, \\
\delta c_{9}-\frac{\delta v^{2}}{v^{2}} & =\frac{a_{C}^{2}}{4} \Delta_{\varepsilon} .
\end{aligned}
$$

It follows that the $\pi$ wave function renormalization has no divergent part whenever $a_{C}^{2}=b_{C}$, which happens for instance in the SM case $\left(a_{C}=b_{C}=1\right)$. Note as well that the absence of a constant term in eq. (6.19) translates into no GB mass being induced at 1-loop level, as expected by chiral symmetry at any loop order. As shown below, this will not be the case of the $h$ particle, not being an exact GB. Furthermore, there is a $p^{4}$ divergent term in the amplitude; which demands the presence stems from the of a $\square \pi \square \pi$ counterterm in the $\mathcal{L}_{4}$ Lagrangian. This is a consequence of the $h-\pi-\pi$ coupling $a_{C}$, which is an entire new feature compared to the nonlinear $\sigma$ model renormalization (where there was no $h$ ).

Turning to the Higgs particle $h$, the diagrams contributing to its self-energy are shown in Fig. 6.3, with the divergent part and the required counterterm structure given by

$$
\begin{aligned}
\Pi_{\text {div }}\left(p^{2}\right) & =p^{4} \frac{\left(3 a_{C}^{2}+a_{H}^{2}\right)}{2 v^{2}}+p^{2}\left(-\frac{\mu_{3}}{v} a_{H}+\frac{m_{h}^{2}\left(5 a_{H}^{2}-b_{H}\right)}{v^{2}}\right) \\
& +\left(\frac{1}{2} \mu_{3}^{2}+\frac{1}{2} m_{h}^{2}\left(\lambda-8 \frac{\mu_{3}}{v} a_{H}\right)+\frac{m_{h}^{4}\left(6 a_{H}^{2}-b_{H}\right)}{v^{2}}\right), \\
\Pi_{c t r}\left(p^{2}\right) & =p^{4} \frac{2 \delta c_{\square H}}{v^{2}}+p^{2} \delta_{h}-\delta m_{h}^{2} .
\end{aligned}
$$




$$
h \rightarrow h
$$
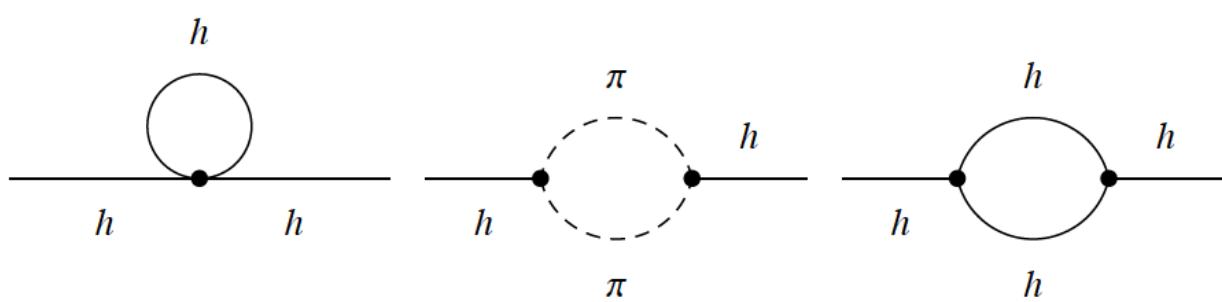

Figure 6.3: Diagrams contributing to the Higgs self-energy.

It follows that the required counterterms are given by

$$
\begin{aligned}
\delta_{h} & =\left[\frac{\mu_{3}}{v} a_{H}+\frac{m_{h}^{2}\left(b_{H}-5 a_{H}^{2}\right)}{v^{2}}\right] \Delta_{\varepsilon}, \\
\delta m_{h}^{2} & =\left[\frac{1}{2} \mu_{3}^{2}+\frac{1}{2} m_{h}^{2}\left(\lambda-8 \frac{\mu_{3}}{v} a_{H}\right)+\frac{m_{h}^{4}\left(6 a_{H}^{2}-b_{H}\right)}{v^{2}}\right] \Delta_{\varepsilon}, \\
\delta c_{\square H} & =-\frac{1}{4}\left(3 a_{C}^{2}+a_{H}^{2}\right) \Delta_{\varepsilon} .
\end{aligned}
$$

This result implies that a non-vanishing $a_{C}$ (as in the SM limit) and/or $a_{H}$ leads to a $p^{4}$ term in the counterterm Lagrangian, requiring a $\square h \square h$ term in $\mathcal{L}_{4}$. In this scheme, a Higgs wave function renormalization is operative only in deviations from the SM with non-vanishing $a_{H}$ and/or $b_{H}$.

\section{3-point functions}

The computations for the 3- and 4-point functions will not be explicitly shown in so much detail as the previous ones; since they are not particularly illuminating. Let us start with the 3-point Green functions. Since there are an odd number of external legs, there has to be always a h particle involved; so there are two possible types.

$h h h$

Let us consider first the $h h h$ amplitude at one loop. The relevant diagrams to be computed are displayed in Fig. 6.4. The results for the counterterms emerging from 


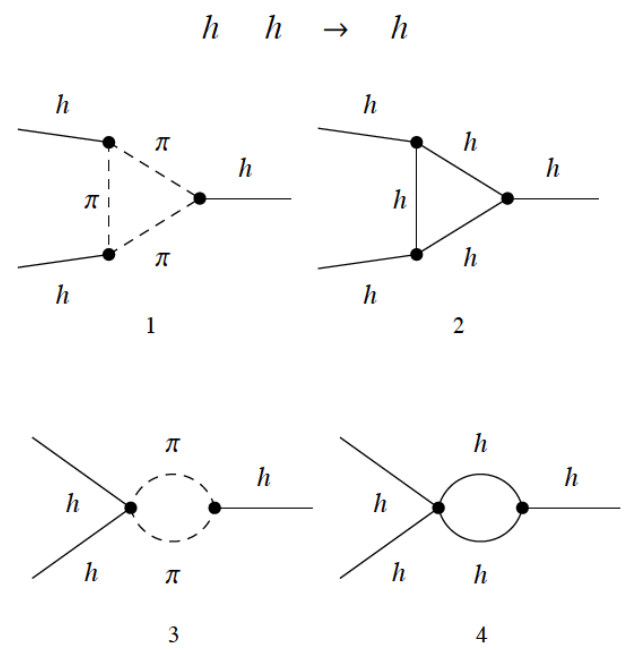

Figure 6.4: Diagrams contributing to the $h h \rightarrow h$ amplitude, not including diagrams obtained by crossing.

$\mathcal{L}_{0}$ and $\mathcal{L}_{2}$ are:

$$
\begin{aligned}
\delta a_{\square H}= & \frac{1}{2}\left(-\frac{3 a_{C} b_{C}}{2}-\frac{a_{H} b_{H}}{2}+3 a_{C}^{3}+a_{H}^{3}\right) \Delta_{\varepsilon}, \\
\delta c_{\Delta H}= & \frac{1}{2}\left(-3 a_{C} b_{C}+3 a_{C}^{3}-a_{H}^{3}\right) \Delta_{\varepsilon}, \\
\delta a_{H}= & {\left[\frac{1}{2}\left(-9 \frac{\mu_{3}}{v} a_{H}^{2}+\lambda a_{H}+2 \frac{\mu_{3}}{v} b_{H}\right)+a_{H}\left(15 a_{H}^{2}-7 b_{H}\right) \frac{m_{h}^{2}}{v^{2}}\right] \Delta_{\varepsilon}, } \\
\delta \mu_{3}= & {\left[\frac{3}{2} \mu_{3}\left(\lambda-4 \frac{\mu_{3}}{v} a_{H}\right)+6\left(6 \mu_{3} a_{H}^{2}-\lambda v a_{H}-\mu_{3} b_{H}\right) \frac{m_{h}^{2}}{v^{2}}\right.} \\
& \left.+6 a_{H}\left(3 b_{H}-8 a_{H}^{2}\right) \frac{m_{h}^{4}}{v^{3}}\right] \Delta_{\varepsilon},
\end{aligned}
$$

As $h$ behaves as a generic singlet, the vertices involving uniquely external $h$ legs which appear in the Lagrangian of Eq. (6.3) will span all possible momentum structures that can result from one-loop amplitudes. Hence, as we will see also in the case of $h h h h$, all divergences could be easily absorbed by the counterterms. As seen below, this will not be the case of processes involving GBs.

$\pi \pi h$

The diagrams for $\pi \pi h$ amplitudes are shown in figure 6.5. The explicit contributions 


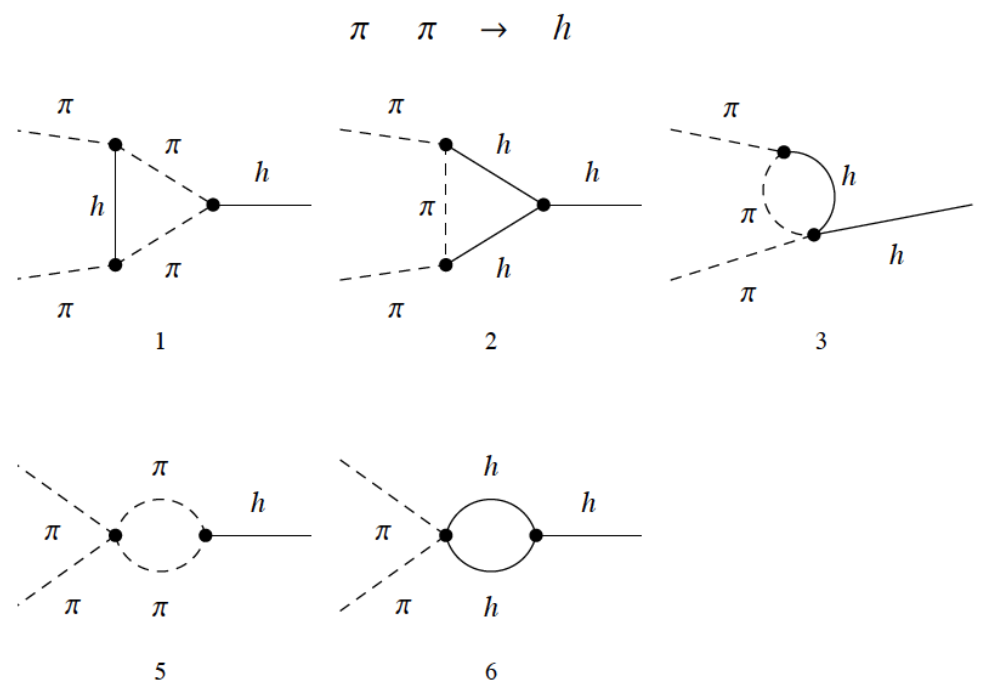

Figure 6.5: Diagrams contributing to the $\pi \pi \rightarrow h$ scattering amplitude, not including diagrams obtained by crossing.

to the counterterms are:

$$
\begin{aligned}
& \delta a_{C}=\frac{1}{2}\left(a_{C}^{2}-b_{C}\right)\left[2\left(a_{C}+2 a_{H}\right) \frac{m_{h}^{2}}{v^{2}}-\frac{\mu_{3}}{v}\right] \Delta_{\varepsilon}, \\
& \delta c_{7}=\frac{1}{4}\left(-a_{H} b_{C}+a_{C}^{2} a_{H}-a_{C}^{3}-2 a_{C}\right) \Delta_{\varepsilon} \\
& \delta a_{9}=-\frac{1}{8} a_{C}\left(a_{C} a_{H}+a_{C}^{2}-b_{C}\right) \Delta_{\varepsilon} \\
& \delta c_{10}=\frac{1}{2} a_{C}\left(-a_{C} a_{H}+a_{C}^{2}+b_{C}\right) \Delta_{\varepsilon} .
\end{aligned}
$$

From these expressions, it can be seen that neither $\delta a_{C}$ nor $\delta a_{9}$ are induced in the SM limit $\left(a_{C}=b_{C}=1, a_{H}=0\right)$. Furthermore, chiral symmetry restricts the possible structures spanned by the pure $\pi$ and $h-\pi$ operators. Because of this, it turns out that part of the divergent amplitude induced by the last diagram in Fig. 6.5 cannot be cast as a function of the $\mathcal{L}_{2}$ and $\mathcal{L}_{4}$ operators; that is, it cannot be reabsorbed by chiral-invariant counterterms. Moreover, its coefficient depends on the pion parametrization used. As a result, an apparent chiral non-invariant divergence (NID) has been identified; which should thus be absorbed by a chiralnon-invariant operator:

$$
\mathcal{O}_{1}^{\mathrm{NID}}=-a_{C}\left(\frac{3}{2}+5 \eta\right) \frac{\Delta_{\varepsilon}}{v^{3}} \pi \square \pi \square h .
$$

NIDs are an artifact of the apparent breaking of chiral symmetry when the oneloop analysis is treated in perturbation theory [147] and have no physical impact; since they vanish for on-shell amplitudes. While long ago NIDs had been isolated in perturbative analysis of four-pion vertices in the non-linear sigma model [146], the result obtained here is a new type of NIDs: a three-point function involving the Higgs particle. See below for more details on their treatment.

It is interesting to note that the renormalization conditions of all physical parameters are independent of the choice of $\mathbf{U}$ parametrization, as they should; while NIDs 
exhibit instead an explicit $\eta$ dependence. This pattern will be also present in the renormalization of 4-point functions, described below.

\section{4-point functions}

hhhh

Let us start by the pure- $h$ case. The relevant diagrams contributing to this amplitude are shown in Fig. 6.6. Applying the renormalization procedure for these amplitudes is straightforward and yields:

$$
\begin{gathered}
\delta b_{H}=\left[\frac{1}{2}\left(\frac{\mu_{3}}{v}\left(-40 a_{H} b_{H}+84 a_{H}^{3}\right)-13 \lambda a_{H}^{2}+3 \lambda b_{H}\right)\right. \\
\left.+\left(87 a_{H}^{2} b_{H}-120 a_{H}^{4}-7 b_{H}^{2}\right) \frac{m_{h}^{2}}{v^{2}}\right] \Delta_{\varepsilon}, \\
\delta b_{\square H}=\frac{1}{4}\left[-3\left(4 a_{H}^{4}+b_{C}^{2}\right)+30 a_{C}^{2} b_{C}+10 a_{H}^{2} b_{H}-36 a_{C}^{4}-b_{H}^{2}\right] \Delta_{\varepsilon}, \\
\delta a_{\Delta H}=-\frac{3}{4}\left(-7 a_{C}^{2} b_{C}+a_{H}^{2} b_{H}+6 a_{C}^{4}-2 a_{H}^{4}+b_{C}^{2}\right) \Delta_{\varepsilon}, \\
\delta c_{D H}=\left[-\frac{3}{4}\left(a_{C}^{2}-b_{C}\right)^{2}-\frac{a_{H}^{4}}{4}\right] \Delta_{\varepsilon}, \\
\delta \lambda=\left\{\frac{3}{2 v^{2}}\left[8 \mu_{3}^{2}\left(6 a_{H}^{2}-b_{H}\right)-16 \lambda \mu_{3} v a_{H}+\lambda^{2} v^{2}\right]\right. \\
-12\left(-12 \mu_{3} a_{H} b_{H}+32 \mu_{3} a_{H}^{3}-6 \lambda v a_{H}^{2}+\lambda v b_{H}\right) \frac{m_{h}^{2}}{v^{3}} \\
\left.+6\left(-48 a_{H}^{2} b_{H}+80 a_{H}^{4}+3 b_{H}^{2}\right) \frac{m_{h}^{4}}{v^{4}}\right\} \Delta_{\varepsilon} .
\end{gathered}
$$

$\pi \pi h h$

The relevant diagrams for this case are displayed in Fig. 6.7, and the required counterterms are:

$$
\begin{aligned}
\delta b_{C}=\frac{1}{2}\left(a_{C}^{2}-b_{C}\right)[ & \left(4 a_{C}+8 a_{H}\right) \frac{\mu_{3}}{v}-\lambda \\
& \left.-2\left(8 a_{C} a_{H}+4 a_{C}^{2}+12 a_{H}^{2}-b_{C}-2 b_{H}\right) \frac{m_{h}^{2}}{v^{2}}\right] \Delta_{\varepsilon}
\end{aligned}
$$




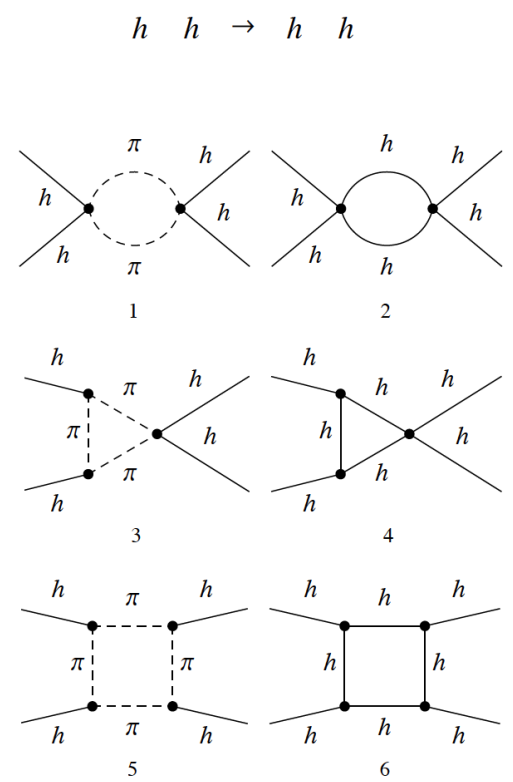

Figure 6.6: Diagrams contributing to the $h h \rightarrow h h$ amplitude, not including diagrams obtained by crossing.

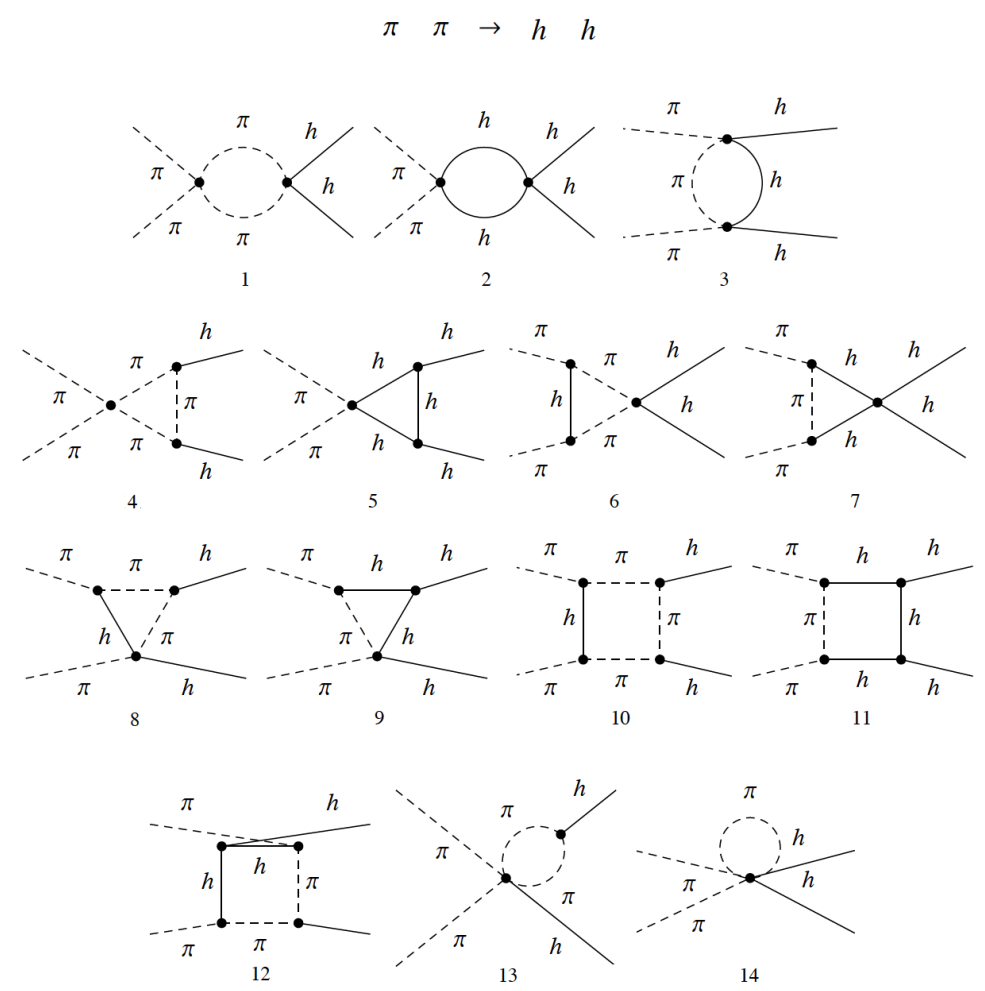

Figure 6.7: Diagrams contributing to the $\pi \pi \rightarrow h h$ amplitude, not including diagrams obtained by crossing. 


$$
\begin{aligned}
& \delta a_{7}= \frac{1}{8}\left[a_{C}^{2}\left(-4 a_{H}^{2}-3 b_{C}+b_{H}+4\right)+2 a_{C} a_{H} b_{C}+b_{C}\left(4 a_{H}^{2}-b_{H}-2\right)+4 a_{C}^{4}\right] \Delta_{\varepsilon}, \\
& \delta c_{8}= \frac{1}{3}\left[a_{C}^{2}\left(a_{H}^{2}+b_{C}\right)-2 a_{C} a_{H} b_{C}-a_{C}^{3} a_{H}+a_{C}^{4}+b_{C}^{2}\right] \Delta_{\varepsilon}, \\
& \delta b_{9}= \frac{1}{4}\left[-a_{C}^{2}\left(-4 a_{H}^{2}+5 b_{C}+b_{H}\right)-4 a_{C} a_{H} b_{C}+4 a_{C}^{3} a_{H}+4 a_{C}^{4}+b_{C}^{2}\right] \Delta_{\varepsilon}, \\
& \delta a_{10}=\frac{1}{4}\left[a_{C}^{2}\left(4 a_{H}^{2}+b_{C}-b_{H}\right)-4 a_{C} a_{H} b_{C}-4 a_{C}^{4}+b_{C}^{2}\right] \Delta_{\varepsilon}, \\
& \delta c_{20}=\frac{1}{12}\left[a_{C}^{2}\left(2 a_{H}^{2}-b_{C}+6\right)+2 a_{C} a_{H} b_{C}\right. \\
&\left.\quad-b_{C}\left(3 a_{H}^{2}+b_{C}+6\right)-2 a_{C}^{3} a_{H}+2 a_{C}^{4}\right] \Delta_{\varepsilon} .
\end{aligned}
$$

Again, this computation shows that the renormalization procedure requires the presence of all possible chiral invariant $\pi \pi h h$ counterterms in the Lagrangian, in the most general case. Furthermore, we have identified new NIDs to be absorbed with:

$$
\begin{aligned}
& \mathcal{O}_{2}^{\mathrm{NID}}=+\left(2 a_{C}^{2}-b_{C}\right)\left(\frac{3}{2}+5 \eta\right) \frac{\Delta_{\varepsilon}}{v^{4}} \boldsymbol{\pi} \square \pi h \square h, \\
& \mathcal{O}_{3}^{\mathrm{NID}}=+\left(a_{C}^{2}-b_{C}\right)\left(\frac{3}{2}+5 \eta\right) \frac{\Delta_{\varepsilon}}{v^{4}} \boldsymbol{\pi} \square \boldsymbol{\pi} \partial_{\mu} h \partial^{\mu} h, \\
& \mathcal{O}_{4}^{\mathrm{NID}}=-2 a_{C}^{2}\left(\frac{3}{2}+5 \eta\right) \frac{\Delta_{\varepsilon}}{v^{4}} \boldsymbol{\pi} \partial_{\mu} \boldsymbol{\pi} \partial^{\mu} h \square h .
\end{aligned}
$$

While these NIDs differ from that for the three-point function in Eq. (6.27) in their counterterm structure; they are all proportional to the factor $(3 / 2+5 \eta)$. Therefore a proper choice of parametrization, i.e. $\eta=-3 / 10$, removes all mixed $h-\pi$ NIDs. Intriguingly, this value of $\eta$ is of no special significance as fas as we know, and in fact, as we will see from the NIDs arising in the $\pi \pi \pi \pi$ case, there is no choice of parametrization that can avoid all non-invariant divergences.

$\pi \pi \pi \pi$

Finally, we consider $\pi \pi \rightarrow \pi \pi$ amplitudes. Diagrams of relevance for the process are displayed in Fig. 6.8. The counterterm amplitude for this case involves not only counterterms unique to this process, $\delta c_{6}$ and $\delta c_{11}$ :

$$
\begin{aligned}
& \delta c_{6}=\frac{1}{48}\left[a_{C}^{2}\left(6 b_{C}-8\right)-2 a_{C}^{4}-3 b_{C}^{2}-2\right] \Delta_{\varepsilon}, \\
& \delta c_{11}=-\frac{1}{12}\left(a_{C}^{2}-1\right)^{2} \Delta_{\varepsilon}
\end{aligned}
$$

but also counterterm coefficients also involved in the GB self-energy renormalization in Eq. (6.21):

$$
\begin{aligned}
& \delta_{\pi}-2 \delta v^{2}=-\left(a_{C}^{2}-b_{C}\right) \frac{m_{h}^{2}}{v^{2}} \Delta_{\varepsilon} \Rightarrow \delta v^{2}=0, \\
& \delta c_{9}=\frac{a_{C}^{2}}{4} \Delta_{\varepsilon} .
\end{aligned}
$$

The previous expressions are a nice check of the computation; since $\delta v^{2}$ is forced to vanish for $\delta_{\pi}$ to be consistent with the renormalization of the GB 2-point function; which also makes $\delta c_{9}$ be in agreement in both $\pi \pi$ and $\pi \pi \pi \pi$ amplitudes. 


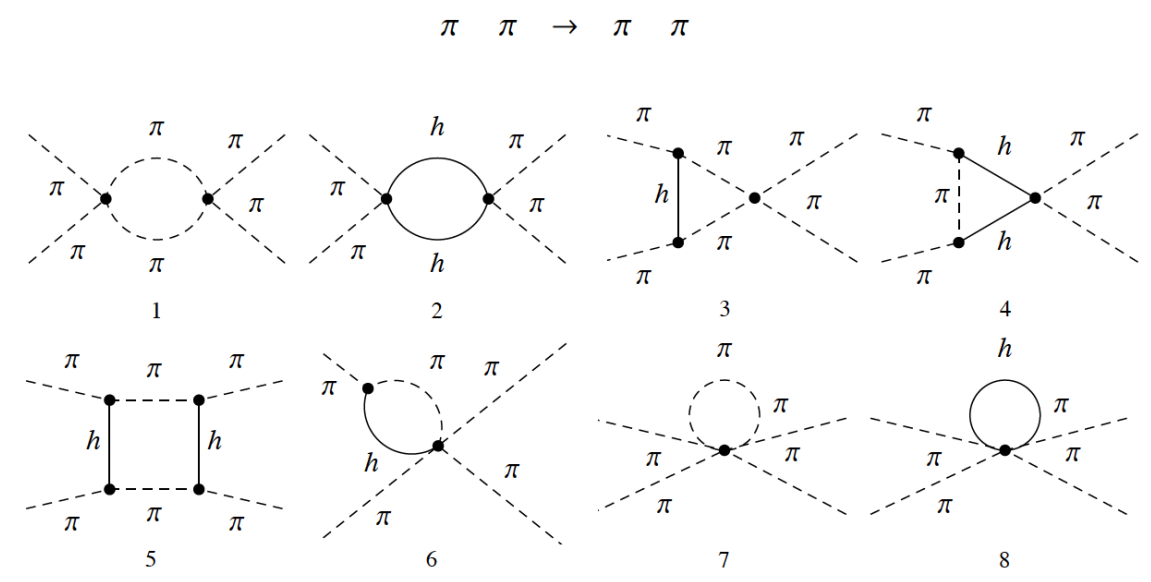

Figure 6.8: Diagrams contributing to the $\pi \pi \rightarrow \pi \pi$ amplitude, not including diagrams obtained by crossing.

We also find NIDs arising in this process; but all of them were already present in the nonlinear $\sigma$ model [146]. They stem from the insertion in the loop of the four-pion vertex (whose coupling depends on $\eta$; see Eq. (6.11)). The NIDs read:

$$
\begin{aligned}
& \mathcal{O}_{5}^{\mathrm{NID}}=+\left(9 \eta^{2}+5 \eta+\frac{3}{4}\right) \frac{\Delta_{\varepsilon}}{v^{4}}(\boldsymbol{\pi} \square \boldsymbol{\pi})^{2}, \\
& \mathcal{O}_{6}^{\mathrm{NID}}=+\left[1+4 \eta+\left(\frac{1}{2}+\eta\right) a_{C}^{2}\right] \frac{\Delta_{\varepsilon}}{v^{4}}(\boldsymbol{\pi} \square \boldsymbol{\pi})\left(\partial_{\mu} \boldsymbol{\pi} \partial^{\mu} \boldsymbol{\pi}\right), \\
& \mathcal{O}_{7}^{\mathrm{NID}}=+2 \eta^{2} \frac{\Delta_{\varepsilon}}{v^{4}} \boldsymbol{\pi}^{2}(\square \boldsymbol{\pi})^{2}, \\
& \mathcal{O}_{8}^{\mathrm{NID}}=+2 \eta\left(a_{C}^{2}-1\right) \frac{\Delta_{\varepsilon}}{v^{4}}\left(\square \boldsymbol{\pi} \partial_{\mu} \boldsymbol{\pi}\right)\left(\boldsymbol{\pi} \partial^{\mu} \boldsymbol{\pi}\right) .
\end{aligned}
$$

As expected, the parametrization freedom - the dependence on the $\eta$ parameter appears only in NIDs, and never on chiral-invariant counterterms. As commented above, no parametrization can remove all pure-GBs NIDs. Note also from the previous expressions, that only $\delta a_{H}$ and $\delta b_{H}$ vanish for an $\mathcal{F}_{H}(h)=1$ in the Lagrangian, in general (with generic $a_{C}$ and $b_{C}$ ).

\section{Comparison with previous works and SM limit}

Previous works on the one-loop renormalization of the scalar sector of the non-linear Lagrangian with a light Higgs have used either the square root parametrization $(\eta=0$ in our parametrization) or the exponential one $(\eta=-1 / 6)$, producing very interesting results. The main difference with this work is that they have concentrated on the analysis of scattering processes relevant for phenomenology, thus doing an on-shell analysis. They also disregard fermionic operators; which in practice this means to neglect all fermion masses, as it have been done here.

As a consequence, our off-shell renormalization procedure requires a larger number of independent four-derivative bosonic operators. However, it is possible to trade those extra operators via the equations of motion by operators from other sectors 
of the theory, including gauge and Yukawa-like interactions. In order to study the theory one has to decide which set is kept, as long as it is complete and independent. In any case, we stress that we have checked that our results reduce to the ones found in [25] (on-shell $h h \rightarrow h h, \pi \pi \rightarrow h h$ and $\pi \pi \rightarrow \pi \pi$; no Higgs potential) and [26] (on-shell $W_{L}^{+} W_{L}^{-} \rightarrow Z_{L} Z_{L}$ ); taking into account all the corresponding assumptions in each computation.

There is another particularity of the off-shell renormalization scheme which deserves to be pointed out. A closer look at the counterterms reveals that, in the SM case, that is

$$
a_{C}=b_{C}=1, \quad a_{H}=b_{H}=0, \quad \mu_{3}=3 \frac{m_{h}^{2}}{v}, \quad \lambda=3 \frac{m_{h}^{2}}{v^{2}},
$$

several BSM operator coefficients computed in this section do not vanish. At first this might look counterintuitive, since the SM is a renormalizable model and thus no new operator should be required by the renormalization procedure to absorb an infinite divergence. However, when calculating physical amplitudes the contribution of these non-vanishing operator coefficients all combine in such a way that the overall BSM contribution indeed cancels in the on-shell processes. The same pattern propagates to the renormalization group equations discussed in Sect. 6.4. This non-triviality in taking the SM limit is the price to pay for using an off-shell renormalization scheme.

\section{Dealing with the apparent non-invariant divergences (NIDs)}

In the previous section it was seen that apparent chiral non-invariant divergences are found in the computation of the 1-loop amplitudes off-shell. As we have already commented, this issue was analyzed long ago in the renormalization of the nonlinear $\sigma$ model $[146,151,158-161]$. Furthermore, it was shown that they do not contribute to on-shell quantities [146]. Thus, one could completely disregard these divergences, as they are unphysical. However, let us explicitly show that this is indeed our case.

By performing a non-linear redefinition the pion field, including space-time derivatives, these divergences can be absorbed [146]. In fact, Lagrangians related by a general local field redefinition $\pi \rightarrow \pi f\left(\pi, h, \partial_{\mu} \pi, \partial_{\mu} h, \ldots\right)$, even when involving derivatives, are equivalent [162-165]. As an aside comment, other alternatives explored in the literature to evade the non-invariant divergences in the non-linear sigma model were a modified background field method $[159,160]$ and the introduction of modified Feynman rules [152].

After the field redefinition, the Lagrangian gets a shift, $\mathcal{L} \rightarrow \mathcal{L}+\delta \mathcal{L}$, so since both Lagrangians must be equivalent, we know the part $\delta \mathcal{L}$ must necessarily be nonphysical. In other words, if a pion field redefinition involving derivatives makes it possible to absorb the non-invariant divergences, and at the same time does not generate any other new structure, then the equivalence between the original and the redefined Lagrangians tells us that such non-invariant terms are unphysical as they do not contribute to the S-matrix and therefore that chiral symmetry remains indeed unbroken. 
We thus perform the following general GB field redefinition, which extends the pion field redefinition considered in [146] to a redefinition with terms containing also the $h$ singlet:

$$
\begin{aligned}
\pi_{i} \rightarrow & \pi_{i}\left[1+\frac{\alpha_{1}}{v^{4}} \boldsymbol{\pi} \square \boldsymbol{\pi}+\frac{\alpha_{2}}{v^{4}} \partial_{\mu} \boldsymbol{\pi} \partial^{\mu} \boldsymbol{\pi}+\frac{\beta}{v^{3}} \square h+\frac{\tilde{\gamma_{1}}}{v^{4}} h \square h+\frac{\gamma_{2}}{v^{4}} \partial_{\mu} h \partial^{\mu} h\right] \\
+ & \frac{\alpha_{3}}{v^{4}} \square \pi_{i}(\boldsymbol{\pi} \boldsymbol{\pi})+\frac{\alpha_{4}}{v^{4}} \partial_{\mu} \pi_{i}\left(\boldsymbol{\pi} \partial^{\mu} \boldsymbol{\pi}\right) .
\end{aligned}
$$

Up to four fields and four derivatives, the following additional terms appear in the Lagrangian (having absorbed all the operators already appearing in the chiral invariant Lagrangian and doing $\left.\gamma_{1}=\tilde{\gamma}_{1}+2 a_{C} \beta\right)$ :

$$
\begin{aligned}
\Delta \mathcal{L}^{\mathrm{NID}}= & -\boldsymbol{\pi} \square \boldsymbol{\pi}\left(\frac{\alpha_{1}}{v^{4}} \boldsymbol{\pi} \square \boldsymbol{\pi}+\frac{\alpha_{2}}{v^{4}} \partial_{\mu} \boldsymbol{\pi} \partial^{\mu} \boldsymbol{\pi}+\frac{\beta}{v^{3}} \square h+\frac{\gamma_{1}}{v^{4}} h \square h+\frac{\gamma_{2}}{v^{4}} \partial_{\mu} h \partial^{\mu} h\right) \\
& -\frac{\alpha_{3}}{v^{4}}(\square \boldsymbol{\pi} \square \boldsymbol{\pi})(\boldsymbol{\pi} \boldsymbol{\pi})-\frac{\alpha_{4}}{v^{4}}\left(\square \boldsymbol{\pi} \partial_{\mu} \boldsymbol{\pi}\right)\left(\boldsymbol{\pi} \partial^{\mu} \boldsymbol{\pi}\right)-\frac{2 a_{C} \beta}{v^{4}}\left(\partial_{\mu} h \square h\right)\left(\boldsymbol{\pi} \partial^{\mu} \boldsymbol{\pi}\right) .
\end{aligned}
$$

We see that the new part of the Lagrangian that has appeared contains counterterms only for processes $h \pi \pi, h h \pi \pi$ and $\pi \pi \pi \pi$, precisely the ones that bear the apparent NIDs. Comparing the terms in $\Delta \mathcal{L}^{\mathrm{NID}}$ with the NID operators found, Eqs. (6.27), (6.30) and (6.33), it follows that by choosing

$$
\begin{array}{rlrl}
\alpha_{1} & =\left(9 \eta^{2}+5 \eta+\frac{3}{4}\right) \Delta_{\varepsilon}, & \beta & =-\left(\frac{3}{2}+5 \eta\right) a_{C} \Delta_{\varepsilon}, \\
\alpha_{2} & =\left[1+4 \eta+\left(\frac{1}{2}+\eta\right) a_{C}^{2}\right] \Delta_{\varepsilon}, & \gamma_{1} & =\left(\frac{3}{2}+5 \eta\right)\left(2 a_{C}^{2}-b_{C}\right) \Delta_{\varepsilon} \\
\alpha_{3} & =2 \eta^{2} \Delta_{\varepsilon}, & \gamma_{2} & =\left(\frac{3}{2}+5 \eta\right)\left(a_{C}^{2}-b_{C}\right) \Delta_{\varepsilon} \\
\alpha_{4} & =2 \eta\left(a_{C}^{2}-1\right) \Delta_{\varepsilon}, &
\end{array}
$$

all 1-loop NIDs are removed away. In conclusion, it has been checked that the addition of extra terms in the Lagrangian, via the redefinition of the pion fields, provides the necessary coefficients for the absorption of the non-invariant divergences, while they do not modify any previous result for the rest of the counterterms and thus no physical effect is added. Moreover, the contribution of the NIDs to on-shell amplitudes vanishes, as expected. This is not always trivially seen when taking the previous results individually. For instance, the contribution of $\mathcal{O}_{4}^{\mathrm{NID}}$ (see Eq. (6.30)) to the $h h \pi \pi$ amplitude on-shell is cancelled by the contribution from the correction to the $h \pi \pi$ vertex, given by $\mathcal{O}_{1}^{\mathrm{NID}}$ (equation (6.27)).

The field redefinitions implemented above to reabsorb the scalar NIDs may indeed be equivalent to the application of the GB equations of motion [166], and contribute to other type of NIDs: for instance those involving simultaneously GBs and fermions, not yet explored. Their exact computation is not called for when exploring the scalar sector and the set of purely scalar counterterms required at one-loop by the theory, which is what is clarified here. 


\subsection{Renormalization Group Equations}

It is straightforward to derive the RGE from the one-loop $\delta c_{i}$ divergent contributions determined in the previous section. The evolution of those Lagrangian coefficients which do not vanish in the SM limit is given by:

$$
\begin{aligned}
& 16 \pi^{2} \frac{d}{d \ln \mu} a_{C}=\frac{1}{2} a_{C}\left[a_{H} \frac{\mu_{3}}{v}+\left(3 b_{C}-5 a_{H}^{2}+b_{H}\right) \frac{m_{h}^{2}}{v^{2}}\right]+a_{C}^{2}\left(\frac{\mu_{3}}{2 v}-2 a_{H} \frac{m_{h}^{2}}{v^{2}}\right) \\
& -\frac{3}{2} a_{C}^{3} \frac{m_{h}^{2}}{v^{2}}-\frac{1}{2 v} b_{C} \mu_{3}+2 a_{H} b_{C} \frac{m_{h}^{2}}{v^{2}} \\
& 16 \pi^{2} \frac{d}{d \ln \mu} b_{C}=b_{C}\left[2 a_{C} \frac{\mu_{3}}{v}+5 a_{H} \frac{\mu_{3}}{v}-\frac{\lambda}{2}-\left(5 a_{C}^{2}+8 a_{H} a_{C}+17 a_{H}^{2}-3 b_{H}\right) \frac{m_{h}^{2}}{v^{2}}\right] \\
& +b_{C}^{2} \frac{m_{h}^{2}}{v^{2}}+\frac{1}{2}\left(-4 a_{C} \frac{\mu_{3}}{v}-8 a_{H} \frac{\mu_{3}}{v}+\lambda\right) a_{C}^{2} \\
& +2\left(2 a_{C}^{2}+4 a_{H} a_{C}+6 a_{H}^{2}-b_{H}\right) a_{C}^{2} \frac{m_{h}^{2}}{v^{2}}, \\
& 16 \pi^{2} \frac{d}{d \ln \mu} m_{h}^{2}=-\frac{1}{2} \mu_{3}^{2}+\left(5 a_{H} \frac{\mu_{3}}{v}-\frac{\lambda}{2}\right) m_{h}^{2}+\left(2 b_{H}-11 a_{H}^{2}\right) \frac{m_{h}^{4}}{v^{2}}, \\
& 16 \pi^{2} \frac{d}{d \ln \mu} \mu_{3}=\frac{1}{2} \mu_{3}\left[\left(-a_{C}^{2}+b_{C}-87 a_{H}^{2}+15 b_{H}\right) \frac{m_{h}^{2}}{v^{2}}-3 \lambda\right]+\frac{15}{2 v} \mu_{3}^{2} a_{H} \\
& +6 a_{H} \lambda \frac{m_{h}^{2}}{v}+6\left(8 a_{H}^{3}-3 a_{H} b_{H}\right) \frac{m_{h}^{4}}{v^{3}}, \\
& 16 \pi^{2} \frac{d}{d \ln \mu} \lambda=\lambda\left[26 a_{H} \frac{\mu_{3}}{v}+\left(14 b_{H}-82 a_{H}^{2}\right) \frac{m_{h}^{2}}{v^{2}}\right]-\frac{3}{2} \lambda^{2} \\
& +12\left(b_{H}-6 a_{H}^{2}\right) \frac{\mu_{3}^{2}}{v^{2}}+48 a_{H}\left(8 a_{H}^{2}-3 b_{H}\right) \mu_{3} \frac{m_{h}^{2}}{v^{3}} \\
& -6\left(80 a_{H}^{4}-48 b_{H} a_{H}^{2}+3 b_{H}^{2}\right) \frac{m_{h}^{4}}{v^{4}} .
\end{aligned}
$$

For completeness, we also include here the resulting RGEs for the rest of coefficients in the Lagrangian:

$$
\begin{aligned}
16 \pi^{2} \frac{d}{d \ln \mu} a_{H}=a_{H} & {\left[\lambda-\left(a_{C}^{2}-b_{C}+17 b_{H}+45 a_{H}^{2}\right) \frac{m_{h}^{2}}{v^{2}}\right] } \\
+ & \left(-12 a_{H}^{2}+2 b_{H}\right) \frac{\mu_{3}}{v} \\
16 \pi^{2} \frac{d}{d \ln \mu} b_{H}=b_{H} & {\left[2\left(-a_{C}^{2}+97 a_{H}^{2}+b_{C}\right) \frac{m_{h}^{2}}{v^{2}}-44 a_{H} \frac{\mu_{3}}{v}+3 \lambda\right] } \\
- & 18 b_{H}^{2} \frac{m_{h}^{2}}{v^{2}}+a_{H}^{2}\left(-13 \lambda+84 a_{H} \frac{\mu_{3}}{v}\right)-240 a_{H}^{4} \frac{m_{h}^{2}}{v^{2}} \\
& 16 \pi^{2} \frac{d}{d \ln \mu} v^{2}=-2\left(a_{C}^{2}-b_{C}\right) m_{h}^{2}
\end{aligned}
$$




$$
\begin{aligned}
16 \pi^{2} \frac{d}{d \ln \mu} c_{6}= & -\frac{1}{24}\left[2+2 a_{C}^{4}+3 b_{C}^{2}-a_{C}^{2}\left(-8+6 b_{C}\right)\right] \\
16 \pi^{2} \frac{d}{d \ln \mu} c_{7}= & -c_{7}\left[\left(a_{C}^{2}-5 a_{H}^{2}-b_{C}+b_{H}\right) \frac{m_{h}^{2}}{v^{2}}+a_{H} \frac{\mu_{3}}{v}\right] \\
& +\frac{1}{2}\left(-2 a_{C}-a_{C}^{3}+a_{C}^{2} a_{H}-a_{H} b_{C}\right)
\end{aligned}
$$

$$
\begin{aligned}
16 \pi^{2} \frac{d}{d \ln \mu} a_{7}=- & a_{7}\left[2\left(a_{C}^{2}-5 a_{H}^{2}-b_{C}+b_{H}\right) \frac{m_{h}^{2}}{v^{2}}+2 a_{H} \frac{\mu_{3}}{v}\right] \\
+\frac{1}{4}[ & 4 a_{C}^{4}+2 a_{C} a_{H} b_{C}+b_{C}\left(-2+4 a_{H}^{2}-b_{H}\right) \\
& \left.+a_{C}^{2}\left(4-4 a_{H}^{2}-3 b_{C}+b_{H}\right)\right],
\end{aligned}
$$

$$
\begin{aligned}
16 \pi^{2} \frac{d}{d \ln \mu} c_{8}= & -c_{8}\left[2\left(a_{C}^{2}-5 a_{H}^{2}-b_{C}+b_{H}\right) \frac{m_{h}^{2}}{v^{2}}+2 a_{H} \frac{\mu_{3}}{v}\right] \\
& +\frac{2}{3}\left[a_{C}^{4}-a_{C}^{3} a_{H}-2 a_{C} a_{H} b_{C}+b_{C}^{2}+a_{C}^{2}\left(a_{H}^{2}+b_{C}\right)\right],
\end{aligned}
$$

$$
\begin{aligned}
16 \pi^{2} \frac{d}{d \ln \mu} c_{9}= & \frac{a_{C}^{2}}{2}, \\
16 \pi^{2} \frac{d}{d \ln \mu} a_{9}= & -a_{9}\left[\left(a_{C}^{2}-5 a_{H}^{2}-b_{C}+b_{H}\right) \frac{m_{h}^{2}}{v^{2}}+a_{H} \frac{\mu_{3}}{v}\right] \\
& -\frac{1}{2} a_{C}\left(a_{C}^{2}+a_{C} a_{H}-b_{C}\right), \\
16 \pi^{2} \frac{d}{d \ln \mu} b_{9}= & -b_{9}\left[2\left(a_{C}^{2}-5 a_{H}^{2}-b_{C}+b_{H}\right) \frac{m_{h}^{2}}{v^{2}}+2 a_{H} \frac{\mu_{3}}{v}\right] \\
& +\frac{1}{2}\left[4 a_{C}^{4}+4 a_{C}^{3} a_{H}-4 a_{C} a_{H} b_{C}+b_{C}^{2}+a_{C}^{2}\left(4 a_{H}^{2}-5 b_{C}-b_{H}\right)\right],
\end{aligned}
$$

$$
\begin{aligned}
16 \pi^{2} \frac{d}{d \ln \mu} c_{10}= & -c_{10}\left[\left(a_{C}^{2}-5 a_{H}^{2}-b_{C}+b_{H}\right) \frac{m_{h}^{2}}{v^{2}}+a_{H} \frac{\mu_{3}}{v}\right] \\
& +a_{C}\left(a_{C}^{2}-a_{C} a_{H}+b_{C}\right), \\
16 \pi^{2} \frac{d}{d \ln \mu} a_{10}= & -a_{10}\left[2\left(a_{C}^{2}-5 a_{H}^{2}-b_{C}+b_{H}\right) \frac{m_{h}^{2}}{v^{2}}+2 a_{H} \frac{\mu_{3}}{v}\right] \\
& +\frac{1}{2}\left(-4 a_{C}^{4}-4 a_{C} a_{H} b_{C}+b_{C}^{2}+a_{C}^{2}\left(4 a_{H}^{2}+b_{C}-b_{H}\right)\right),
\end{aligned}
$$




$$
\begin{aligned}
16 \pi^{2} \frac{d}{d \ln \mu} c_{11}= & -\frac{1}{6}\left(a_{C}^{2}-1\right)^{2}, \\
16 \pi^{2} \frac{d}{d \ln \mu} c_{20}= & -c_{20}\left[2\left(a_{C}^{2}-5 a_{H}^{2}-b_{C}+b_{H}\right) \frac{m_{h}^{2}}{v^{2}}+2 a_{H} \frac{\mu_{3}}{v}\right] \\
& +\frac{1}{6}\left[2 a_{C}^{4}-2 a_{C}^{3} a_{H}+a_{C}^{2}\left(6+2 a_{H}^{2}-b_{C}\right)+2 a_{C} a_{H} b_{C}\right. \\
& \left.-b_{C}\left(6+3 a_{H}^{2}+b_{C}\right)\right],
\end{aligned}
$$

$$
\begin{aligned}
16 \pi^{2} \frac{d}{d \ln \mu} b_{\square H}= & -b_{\square H}\left[4\left(a_{C}^{2}-5 a_{H}^{2}-b_{C}+b_{H}\right) \frac{m_{h}^{2}}{v^{2}}+4 a_{H} \frac{\mu_{3}}{v}\right] \\
& -18 a_{C}^{4}-6 a_{H}^{4}+15 a_{C}^{2} b_{C}-\frac{3 b_{C}^{2}}{2}+5 a_{H}^{2} b_{H}-\frac{b_{H}^{2}}{2}
\end{aligned}
$$

$$
\begin{aligned}
16 \pi^{2} \frac{d}{d \ln \mu} c_{\Delta H}= & -c_{\Delta H}\left[3\left(a_{C}^{2}-5 a_{H}^{2}-b_{C}+b_{H}\right) \frac{m_{h}^{2}}{v^{2}}+3 a_{H} \frac{\mu_{3}}{v}\right] \\
& +3 a_{C}^{3}-a_{H}^{3}-3 a_{C} b_{C},
\end{aligned}
$$

$$
\begin{aligned}
16 \pi^{2} \frac{d}{d \ln \mu} a_{\Delta H}= & -a_{\Delta H}\left[4\left(a_{C}^{2}-5 a_{H}^{2}-b_{C}+b_{H}\right) \frac{m_{h}^{2}}{v^{2}}+4 a_{H} \frac{\mu_{3}}{v}\right] \\
& -\frac{3}{2}\left(6 a_{C}^{4}-2 a_{H}^{4}-7 a_{C}^{2} b_{C}+b_{C}^{2}+a_{H}^{2} b_{H}\right), \\
16 \pi^{2} \frac{d}{d \ln \mu} c_{D H}= & -c_{D H}\left[4\left(a_{C}^{2}-5 a_{H}^{2}-b_{C}+b_{H}\right) \frac{m_{h}^{2}}{v^{2}}+4 a_{H} \frac{\mu_{3}}{v}\right] \\
& -\frac{1}{2}\left[a_{H}^{4}+3\left(a_{C}^{2}-b_{C}\right)^{2}\right] .
\end{aligned}
$$

Note that in the RGE for the Higgs quartic self-coupling $\lambda$, Eq. (6.42), some terms are weighted by numerical factors of $\mathcal{O}(100)$. This suggests that if a BSM theory results in small couplings for $a_{H}$ and $b_{H}$, those terms could still induce measurable phenomenological consequences. Nevertheless, physical amplitudes will depend on a large combination of parameters, which might yield cancellations or enhancements as pointed out earlier, and only a more thorough study can lead to firm conclusions. 


\section{Chapter 7}

\section{Complete renormalization}

In this chapter we present the complete computation of the renormalization of the HEFT Lagrangian [167]. In the previous chapter, we presented the renormalization of the off-shell Green functions from the scalar sector of the Lagrangian. Despite the automation tools used in Mathematica to perform the computation, there was still a large amount of diagrams to calculate. In order to avoid this, we will use here methods based in the Covariant Derivative Expansion (CDE) [168, 169], whose potential to trivialize one-loop computations has recently been explored in different works [170-174].

We will also use the geometric formulation of the bosonic sector of the HEFT Lagrangian developed in Refs. [148, 149]. In those references and also Ref. [28] the effective one-loop Lagrangian is determined for the bosonic sector with only scalars in the internal loops. We extend here those studies and consider all possible fields in the loops. In this way we are able to explicitly determine the structures required as counterterms to renormalize the LO custodial-invariant HEFT Lagrangian and establish the complete set of one-loop RGEs.

\subsection{A gauge and Yukawa theory for a manifold of scalars}

Let us consider a manifold parametrized by a set of scalar fields $\phi^{i}$ and metric $G_{i j}$, fermions fields $\psi$ and a gauge symmetry group acting on both. In order to handle the gauge symmetries, we will work in terms of the isometries, which here will be the symmetries of the scalar kinetic terms. The isometries are generated by Killing vectors, $t(\phi)$ :

$$
\delta \phi^{i}=\theta^{A} t_{A}^{i}(\phi)
$$

where $A$ is an index labelling the isometries and $\theta$ is an infinitesimal parameter. In order to preserve the metric of the manifold, the Killing vectors satisfy the following 
condition

$$
t_{A}^{k} \frac{\partial G_{i j}}{\partial \phi^{k}}+G_{k j} \frac{\partial t_{A}^{k}}{\partial \phi^{i}}+G_{i k} \frac{\partial t_{A}^{k}}{\partial \phi^{j}}=0,
$$

When part or the whole symmetry is gauged we promote the corresponding infinitesimal parameters $\theta \rightarrow \theta(x)$, so the derivative of the scalar field needs to be promoted to the covariant derivative, which has the form

$$
d_{\mu} \phi^{i}=\partial_{\mu} \phi^{i}+A_{\mu}^{B} t_{B}^{i}(\phi)
$$

where $t$ is assumed to contain the gauge coupling constant and $A_{\mu}$ is the gauge field, transforming as

$$
\delta A_{\mu}^{B}=-\partial_{\mu} \theta^{B}-f_{C D}^{B} \theta^{C} A_{\mu}^{D},
$$

in order to leave $G_{i j} d_{\mu} \phi^{i} d_{\mu} \phi^{j}$ invariant, and the $f_{A B}^{C}$ constants are defined as

$$
t_{A}^{k} \frac{\partial t_{B}^{i}}{\partial \phi^{k}}-t_{B}^{k} \frac{\partial t_{A}^{i}}{\partial \phi^{k}}=f_{A B}^{C} t_{C}^{i}
$$

The space of scalar fields has a non-trivial metric and thus we should also define a covariant derivative in the field space:

$$
\begin{aligned}
\nabla_{i} s & =\frac{\partial s}{\partial \phi^{i}} \\
\nabla_{i} v^{j} & =\frac{\partial v^{j}}{\partial \phi^{i}}+\Gamma_{i k}^{j} v^{k}
\end{aligned}
$$

where $\Gamma_{j k}^{i}$ are the Christoffel symbols defined in terms of the metric as

$$
\Gamma_{j k}^{i}=\frac{1}{2} G^{i m}\left(\frac{\partial G_{m j}}{\partial \phi^{k}}+\frac{\partial G_{m k}}{\partial \phi^{j}}-\frac{\partial G_{j k}}{\partial \phi^{m}}\right) .
$$

Finally, it is also useful to introduce the covariant derivative for a upper-index object in field space containing also the gauge interaction:

$$
\left(D_{\mu} \eta\right)^{i}=\left(\partial_{\mu} \eta^{i}+\Gamma_{k j}^{i} d_{\mu} \phi^{k} \eta^{j}\right)+A_{\mu}^{\beta} t_{\beta, j}^{i} \eta^{j}
$$

On the other hand, in the fermion sector the kinetic term respects a $U(n)$ symmetry for $n$ species of Weyl fermions. We can write them inside Dirac fermions $\psi$ transforming as

$$
\delta \psi=i \theta^{A} T_{A}^{\psi} \psi
$$

where $T_{A}^{\psi}$ are the corresponding Hermitian generators for the gauge transformation and they are assumed to contain the gauge coupling and the appropriate projectors $P_{L, R}$ onto the different chiralities. The covariant derivative acting on a fermion then reads

$$
D_{\mu} \psi=\left(\partial_{\mu}+i T_{B}^{\psi} A_{\mu}^{B}\right) \psi
$$

Finally, the Lagrangian is

$$
\mathcal{L}=\frac{G_{i j}}{2} d^{\mu} \phi^{i} d_{\mu} \phi^{j}+\bar{\psi}(i \not D-\mathcal{Y}(\phi)) \psi-\frac{1}{4} A_{\mu \nu} A^{\mu \nu}-V(\phi),
$$


where $A_{\mu \nu}$ is the field strength and $V(\phi)$ is the scalar potential. It contains a Yukawa interaction given by $\mathcal{Y}(\phi)$. Flavor and gauge indices have been omitted for simplicity.

From (7.3) and (7.9), the subgroup of isometries and $U(n)$ fermion symmetries respected by the potential and the Yukawa interactions satisfy:

$$
\begin{gathered}
\theta^{A} t_{A}^{i} \frac{\partial V}{\partial \phi^{i}}=0 \\
\theta^{A} t_{A}^{i} \frac{\partial \mathcal{Y}}{\partial \phi^{i}}-i T_{A}^{\psi} \theta^{A} \mathcal{Y}+i \mathcal{Y} T_{A}^{\psi} \theta^{A}=0
\end{gathered}
$$

\section{One-loop renormalization}

To briefly explain the procedure to compute the one-loop corrections; let us take a scalar theory with action $S[\phi]$. The S-matrix can be computed from the n-point Green functions $G^{(n)}$ by taking functional derivatives with respect to the source function $J$ of the generating functional

$$
Z[J]=\int \mathcal{D} \phi e^{i\left(S[\phi]+\int d x J(x) \phi(x)\right)}
$$

Connected diagrams are actually given by the functional derivatives of $W[J]=$ $-i \log Z[J]$; while $1 P I$ Green functions are generated by a functional denominated the effective action, which is defined as the Legendre transformation of $W[J]$ :

$$
\Gamma[\Phi]=W[J]-\int d x J \Phi
$$

where

$$
\Phi \equiv \frac{\delta W}{\delta J}
$$

corresponds to the vacuum expectation value of $\phi$ in the presence of the source $J$.

In the background field method [175], the effective action can be calculated by expanding a quantum field $\Phi$ around a classical "background"

$$
\phi \rightarrow \Phi+\eta
$$

where $\Phi$ is the solution to the classical equations of motion:

$$
\left.\frac{\delta S}{\delta \phi}\right|_{\phi=\Phi}+J=0
$$

It can be proved that the solution to the equations of motion is also the vev of $\phi$, of Eq. (7.16) (and thus the same notation has been used), see for instance chapter 21 in [176]. Then expanding around the background, the generating functional is, schematically:

$$
e^{i \Gamma}=\int \mathcal{D} \eta \exp \left[i S[\Phi]+\left.i \frac{\eta^{2}}{2} \frac{\delta^{2} S}{\delta \phi^{2}}\right|_{\Phi}+\ldots\right]
$$


where the integral has a Gaussian form, so neglecting higher-order corrections, it can be computed to be proportional to

$$
e^{i \Gamma} \approx e^{i S[\Phi]}\left(\operatorname{det}\left(-\left.\frac{\delta^{2} S[\Phi]}{\delta \phi^{2}}\right|_{\Phi}\right)\right)^{-1 / 2},
$$

so the effective action, at one-loop is

$$
S_{\text {eff }} \equiv \Gamma[\Phi]=S[\Phi]+\frac{i}{2} \log \left[\operatorname{det}\left(-\left.\frac{\delta^{2} S}{\delta \phi^{2}}\right|_{\Phi}\right)\right]
$$

Since in general it is necessary to compute the determinant of the second variation of the action, we will rewrite it in a quadratic form in the variations of the fields, by completing squares via field redefinitions that do not change the measure. For the Lagrangian of Eq. (7.11), this reads:

$$
\begin{aligned}
\delta^{2} S= & \delta \bar{\psi} \Pi_{\psi} \delta \psi-\frac{1}{2} \delta A_{\mu} \hat{\Pi}_{A}^{\mu \nu} \delta A_{\nu} \\
& -\frac{1}{2} \delta \phi\left\{\Pi_{\phi}+2 \bar{\psi} \nabla \mathcal{Y} \Pi_{\psi}^{-1} \nabla \mathcal{Y} \psi-\hat{t}_{l} \hat{\Pi}_{A}^{-1} \hat{t}_{r}\right\} \delta \phi
\end{aligned}
$$

where the different pieces are:

$$
\begin{aligned}
& \Pi_{\psi}=i \not D-\mathcal{Y}, \\
& \left(\Pi_{\phi}\right)_{i j}=D_{i j}^{2}+R_{k i l j} d \phi^{k} d \phi^{l}+\nabla_{i j}^{2}(V+\bar{\psi} \mathcal{Y} \psi), \\
& \left(\hat{\Pi}_{A}\right)_{B C}^{\mu \nu}=\left(\Pi_{A}\right)_{B C}^{\mu \nu}+\left(\bar{\psi} \gamma^{\mu} T_{B}^{\psi} \Pi_{\psi}^{-1} \gamma^{\nu} T_{C}^{\psi} \psi+h . c .\right), \\
& \quad\left(\Pi_{A}\right)_{B C}^{\mu \nu}=-\eta^{\mu \nu} \delta_{B C} D^{2}-2 f_{B C}^{D} F_{D}^{\mu \nu}-\eta^{\mu \nu} t_{B} t_{C}, \\
& \left(\hat{t}_{l}\right)_{i}^{\mu B}=-t_{i}^{B} D^{\mu}+2 d^{\mu} \phi \nabla_{i} t^{B}-\left(\bar{\psi} \nabla_{i} \mathcal{Y} \Pi_{\psi}^{-1} \gamma^{\mu} T^{\psi B} \psi+\text { h.c. }\right), \\
& \left(\hat{t}_{r}\right)_{i}^{\mu B}=t_{i}^{B} D^{\mu}+d^{\nu} \phi \nabla_{i} t^{B}-\left(\bar{\psi} \gamma^{\mu} T^{\psi B} \Pi_{\psi}^{-1} \nabla_{i} \mathcal{Y} \psi+\text { h.c. }\right),
\end{aligned}
$$

where contracted indices have been omitted when obvious and, as for the notation, $\eta^{\mu \nu}$ is the Minkowski metric and $R_{i j k l}$ is the Riemann curvature tensor of the scalar manifold* $\left[T_{B}^{\psi}, T_{C}^{\psi}\right]=i f_{B C}^{A} T_{A}^{\psi}$ are the structure constants of the gauge symmetry. When the covariant derivative (see Eq. (7.10)) acts on the adjoint representation; i. e. in $D_{\mu}\left(\delta A_{\nu}^{B}\right)=\left(\partial_{\mu} \delta_{C}^{B}+i\left(T_{D}^{\mathcal{G}}\right)_{C}^{B} A_{\mu}^{D}\right) \delta A_{\nu}^{C}$, then $\left(T_{D}^{\mathcal{G}}\right)_{C}^{B}=i f_{D C}^{B}$. From (7.6), the second covariant derivative in scalar field space acting on the potential (or the Yukawa matrix), has the form

$$
\nabla_{i j} V=\frac{\partial^{2} V}{\partial \phi^{i} \partial \phi^{j}}-\Gamma_{i j}^{k} \nabla_{k} V
$$

Furthermore, the logarithm is expanded using the CDE method, which does not break covariant derivative structures (hence the name). In [170,172] the procedure is outlined for different simple Lagrangians. In particular, in [172] they discuss the

*Explicit expressions for the curvature and other geometry related objects can be found in Refs. [148, 150] 
subtleties of adapting the method to theories with cross-terms from fields of different spin-statistics by using a toy Yukawa theory.

The different integrals over momenta arising (see [170-172]) are then evaluated; so, keeping only the divergent pieces, we find:

$$
\begin{aligned}
S_{e f f}= & \frac{1}{32 \pi^{2} \varepsilon}\left[\frac{11}{12} \mathbb{C}_{\mathcal{G}} A_{\mu \nu} A^{\mu \nu}-\frac{1}{2} \operatorname{Tr}\left\{\left(\mathcal{Y}^{\dagger} \mathcal{Y}-i(\not D \mathcal{Y})\right)^{2}+\frac{1}{6 i^{2}}\left(\left[D_{\mu}, D_{\nu}\right]\right)^{2}\right\}\right. \\
& +\frac{1}{2} \operatorname{Tr}\left\{\left(R d \phi^{2}+\nabla^{2}(V+\bar{\psi} \mathcal{Y} \psi)-t t\right)^{2}+\frac{1}{6}\left(\left[D_{\mu}, D_{\nu}\right]\right)^{2}+2\left(t^{A} t^{B}\right)^{2}\right\} \\
& +\bar{\psi} \nabla \mathcal{Y}\left(i \not D+2 \mathcal{Y}^{\dagger}\right) \nabla \mathcal{Y} \psi-\left(i 2 \bar{\psi}(t \nabla \mathcal{Y}) T^{\psi} \psi+h . c .\right) \\
& \left.-2\left(d_{\mu} \phi \nabla_{i} t\right)\left(d^{\mu} \phi \nabla^{i} t\right)+\bar{\psi} T^{\psi}(2 i \not D-8 \mathcal{Y}) T^{\psi} \psi,\right]
\end{aligned}
$$

where we have used dimensional regularization with $d=4-2 \varepsilon$ and the Feynmant'Hooft gauge. Again, indices are not shown when it is not necessary, and some terms have been simplified so the result is more compact; for instance, $R d \phi^{2} \equiv$ $R_{i j k l} d_{\mu} \phi^{j} d^{\mu} \phi^{l} . \quad \mathbb{C}_{\mathcal{G}}$ denotes the Casimir of the adjoint representation: $\sum T_{A}^{\mathcal{G}} T_{A}^{\mathcal{G}}=$ $\mathbb{C}_{\mathcal{G}} \mathbb{1}$. The covariant derivative inside the commutator of the second line is the one acting on an upper-index object in field space of Eq. (7.8) and the trace is evaluated on the scalar space. On the other hand, the covariant derivative in the commutator of the first line is the one acting on a fermion field as in Eq. (7.10), with the trace evaluated in fermion space.

\subsection{One-loop HEFT}

In this section we apply the previous result of Eq. (7.25) to the HEFT. Therefore, the gauge group is $\mathcal{G}_{S M}=S U(3)_{c} \times S U(2)_{L} \times U(1)_{Y}$ and there are six fermion representations, which we group in the following way $(\mathrm{RH} \text { neutrinos are optional })^{\dagger}$ in order to have a more compact notation:

$$
\begin{array}{rlrl}
\psi_{L} & =\left(q_{L}, \ell_{L}\right)^{T}, & \psi_{R} & =\left(u_{R}, d_{R}, \nu_{R}, e_{R}\right)^{T}, \\
\mathrm{Q}_{\psi_{L}} & =\operatorname{diag}\left(\frac{1}{6},-\frac{1}{2}\right), \mathrm{Q}_{\psi_{R}}=\operatorname{diag}\left(\frac{2}{3},-\frac{1}{3}, 0,-1\right),
\end{array}
$$

where $T_{\psi}^{Y}=g^{\prime} Q_{\psi}$ in the gauge derivative of Eq. (7.10); while the non-abelian generators are introduced with the usual conventions $\operatorname{Tr}\left(T_{A} T_{B}\right)=g_{(s)}^{2} \delta_{A B} / 2$ for weak isospin (color).

To describe the scalar sector we will use a similar notation as in $[148,149]$, which we review in the following paragraphs.

As explained in Sect. 3.1, the scalar sector of the SM has a complex scalar doublet $\Phi$, which can be written in terms of four real scalar fields as:

$$
\Phi(x)=\frac{1}{\sqrt{2}}\left(\begin{array}{c}
i \phi_{1}(x)+\phi_{2}(x) \\
\mathrm{h}(x)-i \phi_{3}(x)
\end{array}\right),
$$

\footnotetext{
${ }^{\dagger}$ Here the notation is changed slightly from previous chapters for convenience. Flavor indices will be omitted unless necessary; fermions are now denoted using lowercase, so $\mathrm{Q}$ is used to represent the matrices containing the charges under $U(1)_{Y}$.
} 
where $h$ is the Higgs singlet and $\phi^{a}$ are the three GBs to be eaten by the EW gauge bosons to become massive. The Higgs potential of Eq. (3.5) depends only on $\Phi^{\dagger} \Phi$, i.e. on the modulus of the scalar 4-vector

$$
\phi=\left(\mathrm{h}, \phi_{1}, \phi_{2}, \phi_{3}\right)^{T}
$$

which transforms linearly under the $O(4)$ symmetry of the potential. The minimum of the potential is at

$$
\langle\phi \cdot \phi\rangle=\left\langle\mathrm{h}^{2}+\phi_{1}^{2}+\phi_{2}^{2}+\phi_{3}^{2}\right\rangle=v^{2}
$$

a three-sphere $S^{3}$ of radius $v$.

It is convenient to rewrite the fields as polar coordinates by introducing a 4-dimensional unit vector in $\mathbb{R}^{4}, u^{i}(\varphi)(i=0,1,2,3)$, parametrized by three angular variables $\varphi^{a \ddagger}(a=1,2,3)$ :

$$
\left(\begin{array}{c}
\mathrm{h} \\
\phi^{1} \\
\phi^{2} \\
\phi^{3}
\end{array}\right)=h\left(\begin{array}{l}
u^{0}(\varphi) \\
u^{1}(\varphi) \\
u^{2}(\varphi) \\
u^{3}(\varphi)
\end{array}\right)
$$

The potential will then depend only on $h$; while the $\varphi^{a}$ fields will just have derivative couplings. Under an $O(4)$ transformation $h$ remains invariant, while $u$ transforms linearly, as $\phi$. The $O(4)$ transformation preserves $u(\varphi) \cdot u(\varphi)=1$, so only three components of the vector are free. The three unconstrained fields will then transform non-linearly under $O(4)$. As discussed in Sect. 6.2, many parametrizations of $u(\varphi)$ are possible as long as the non-linear constraint is satisfied. Since the vacuum is chosen to be at $\langle\phi\rangle=(v, 0,0,0)^{T}, u$ is such that $u^{j}(0)=\delta_{0}^{j}$.

The SM EW gauge group $\mathcal{G}_{S M}$ is a subgroup of the global symmetry group of the Higgs sector: $\mathcal{G}_{E W} \subset \mathcal{G}=O(4) \sim S U(2) \times S U(2)$. The vev $\langle\phi\rangle$ spontaneously breaks $\mathcal{G}$ to the unbroken subgroup $\mathcal{H}=O(3)$; so $\phi^{a}$ and h transform under the unbroken $O(3)$ as a triplet and singlet, respectively. The EW gauge symmetry contained in $\mathcal{G}$ is just partially contained in $\mathcal{H}$, being the overlap between them the unbroken electromagnetic gauge symmetry $\mathcal{H}_{g}=U(1)_{\text {em }}$.

$O(3)$ is the custodial symmetry (it has the same Lie algebra as $S O(3)$, which is isomorphic to the Lie algebra of $S U(2))$ and thus $O(3) \sim S U(2)_{V}$. Therefore, the unbroken symmetry leads to the relation $M_{W}=M_{Z} \cos \theta_{W}$, as predicted in the SM; see Sect. 5.2. For this reason we are assuming custodial symmetry throughout this chapter and thus a good approximation for the GBs manifold is the 3-sphere $\mathcal{G} / \mathcal{H} \simeq S^{3}$.

The HEFT scalar sector will thus have four fields: $h$ and the GBs $\varphi^{a}$, arranged as $\phi=\left(h, \varphi^{a}\right)$, the spherical polar coordinates of a curved scalar manifold with metric:

$$
G_{i j}=\operatorname{diag}\left(1, F(h)^{2} g_{a b}(\varphi)\right)
$$

\footnotetext{
${ }^{\ddagger}$ We will treat $\varphi^{a}$ as angular variables with no mass dimensions. The $\varphi^{a}$ fields in this chapter are equivalent to the $\pi^{a} / v$ fields appearing in the previous chapter.

§See Ref. [149] for a discussion on the breaking of custodial symmetry.
} 
where $g_{a b}$ is the $O(3)$ invariant metric on the GB manifold:

$$
g_{a b}(\varphi)=\partial_{a} u(\varphi) \cdot \partial_{b} u(\varphi)
$$

with $\partial_{a} \equiv \partial / \partial \varphi^{a}$.

As it was introduced in the first section of this chapter, gauge symmetries will be discussed in terms of Killing vectors of the scalar manifold. In order to get the explicit form for the Killing vector, $t_{A}$, it is convenient to write the scalar field transformation of Eq. (7.1) as

$$
\delta \phi^{i}=\theta^{A} \hat{T}_{A}^{i j} \phi^{j}
$$

with $\hat{T}_{A}$ antisymmetric real matrices satisfying $\operatorname{Tr}\left(\hat{T}_{A} \hat{T}_{B}\right)=-\left(g^{(\prime)}\right)^{2} \delta_{A B}$ for weak isospin (hypercharge). With all the above, taking into account the radial field $h$ does not transform under the $\mathcal{G}$ group, the explicit expression for the Killing vector is

$$
t_{A}=\left(0, g^{a b} \partial_{b} u \cdot \hat{T}_{A} \cdot u\right)
$$

Finally, in order to write the scalar couplings to fermions it is also convenient to introduce the unitary matrix $U(\varphi)$ :

$$
U U^{\dagger}=U^{\dagger} U=\mathbb{1}, \quad U(\varphi)=\hat{\tau} \cdot u(\varphi),
$$

where $\hat{\tau} \equiv\left\{\mathbb{1}, i \tau^{a}\right\}$, with $\tau^{a}$ the Pauli matrices.

With all these definitions, the Lagrangian reads

$$
\begin{aligned}
\mathcal{L} & =\frac{1}{2} \partial_{\mu} h \partial^{\mu} h+\frac{1}{2} F(h)^{2} d \varphi^{2}-\frac{1}{4} A_{\mu \nu} A^{\mu \nu}-V(h) \\
& +\bar{\psi}\left(i \not D-\mathcal{U}(\varphi) \mathcal{Y}(h) P_{R}-\mathcal{Y}^{\dagger}(h) \mathcal{U}^{\dagger}(\varphi) P_{L}\right) \psi,
\end{aligned}
$$

where $\mathcal{Y}(h)=\operatorname{diag}\left(Y_{u}(h), Y_{d}(h), Y_{\nu}(h), Y_{e}(h)\right)$, with $Y_{I}(h)(I=u, d, \nu, e)$ as $3 \times 3$ matrices in flavor space $\left(Y_{\nu}\right.$ is optional $)$ and $\mathcal{U}=\mathbb{1} \otimes U=\operatorname{diag}(U, U) . A_{\mu \nu}$ represents schematically the different field strengths in the SM, see Eq. (1.1).

The Higgs potential $V(h)$ is a function of the $h$ field as in Eq. (6.3). It is important to note that the field $h$ denotes the Higgs excitation around the true EW vacuum and therefore the potential has to satisfy at any order that

$$
V^{\prime}(0)=0,
$$

where the prime represents a derivative with respect to $h . \quad F(h)$ and $Y_{I}(h)$ are generic functions of the singlet $h$, as explained in Sect. 5.3,

$$
\begin{aligned}
& F(h)=v\left(1+a \frac{h}{v}+b \frac{h^{2}}{v^{2}}+\ldots\right), \\
& Y_{I}(h)=Y_{I}^{(0)}+Y_{I}^{(1)} \frac{h}{v}+Y_{I}^{(2)} \frac{h^{2}}{v^{2}}+\ldots,
\end{aligned}
$$

where $Y_{I}^{(n)}$ have mass dimension equals one (they contain the Higgs vev that in Sect. 5.3 was written outside) and $F$ satisfies

$$
F(0)=v=246 \mathrm{GeV},
$$


from the measured gauge boson masses.

Finally, for illustration and later use, we leave here the expressions in the SM limit:

$$
\begin{aligned}
& F_{S M}=h+\langle h\rangle, \quad \mathcal{Y}_{S M}=(h+\langle h\rangle) Y_{S M} / \sqrt{2}, \\
& V_{S M}=-\frac{m_{h}^{2}}{2}(h+\langle h\rangle)^{2}+\frac{\lambda}{8}(h+\langle h\rangle)^{4}, \quad\langle h\rangle^{2}=\frac{2 m_{h}^{2}}{\lambda}=v^{2} .
\end{aligned}
$$

\section{Renormalization of the Leading Lagrangian}

The LO Lagrangian in Eq. (7.36) receives corrections in all the terms at one-loop. These corrections are Higgs-singlet field dependent, e.g. $\left(\partial_{\mu} h\right)^{2} \rightarrow Z(h)\left(\partial_{\mu} h\right)^{2}$, except for the gauge bosons kinetic term. The gauge bosons obtain the standard renormalization, as can be read from Eq. (7.25). It is then required, in order to revert the Lagrangian to its original form, to have $h$-dependent renormalization factors. Explicitly, the field redefinitions required to recover canonical kinetic terms are:

$$
\begin{aligned}
\psi_{R} & \rightarrow \psi_{R}-\frac{1_{\varepsilon}}{32 \pi^{2}}\left(\mathbb{C}_{\psi_{R}}^{\mathcal{G}}+\frac{1}{2}\left(3 \frac{\mathcal{Y}^{\dagger} \mathcal{Y}}{F^{2}}+\mathcal{Y}^{\prime \dagger} \mathcal{Y}^{\prime}\right)\right) \psi_{R}, \\
\psi_{L} & \rightarrow \psi_{L}-\frac{1_{\varepsilon}}{32 \pi^{2}}\left(\mathbb{C}_{\psi_{L}}^{\mathcal{G}}+\frac{\mathcal{U}}{2}\left(\mathcal{Y}^{\prime} \mathcal{Y}^{\prime \dagger}-\frac{\mathcal{Y}^{\dagger}}{F^{2}}\right) \mathcal{U}^{\dagger}+\frac{\mathcal{Y} \mathcal{Y}^{\dagger}+\tilde{\mathcal{Y}} \tilde{\mathcal{Y}}^{\dagger}}{F^{2}}\right) \psi_{L}, \\
h \rightarrow & \\
h & -\frac{1_{\varepsilon}}{32 \pi^{2}} \int d h\left[\frac{\left(g^{\prime}\right)^{2}+3 g^{2}}{4}\left(F^{\prime \prime} F-2\left(F^{\prime}\right)^{2}\right)+2 \operatorname{Tr}\left(\mathcal{Y}^{\prime} \mathcal{Y}^{\dagger \prime}\right)-\frac{3 V^{\prime} F^{\prime \prime} F^{\prime}}{F^{2}}\right],
\end{aligned}
$$

where $\tilde{\mathcal{Y}} \equiv(\mathbb{1} \otimes \epsilon) \mathcal{Y}=\operatorname{diag}\left(\epsilon \operatorname{diag}\left(Y_{u}, Y_{d}\right), \epsilon \operatorname{diag}\left(Y_{\nu}, Y_{e}\right)\right)$, with $\epsilon$ the $2 \times 2$ antisymmetric tensor. We denote the divergence by $1_{\varepsilon} \equiv 1 / \varepsilon+\log \mu$. $\mathbb{C}_{\mathcal{R}}^{\mathcal{G}}$ is the Casimir of the representation $\mathcal{R}, \sum_{\mathcal{G}} T_{A}^{\mathcal{R}} T_{A}^{\mathcal{R}}=\mathbb{C}_{\mathcal{R}}^{\mathcal{G}} \mathbb{1}$. For instance, in the case of the $\mathrm{LH}$ quark doublet, the corresponding Casimir is: $\mathbb{C}_{q_{L}}=(1 / 6)^{2} g^{\prime 2}+3 / 4 g^{2}+4 / 3 g_{s}^{2}$. The limits on the integral in the Higgs field redefinition should be chosen so the tadpole vanishes at loop level and thus our expansion is at any order around the true minimum. See the discussion below Eqs. (7.44)-(7.46).

After these redefinitions three terms in the Lagrangian differ from the classical expression: Yukawa couplings, the potential and the kinetic term for the Goldstone bosons $\varphi$. The UV divergent part of these corrections has a scale dependence that we present as customary in the form of a set of renormalization group equations (RGEs). Despite being formally closed, they constitute however an infinite set due to the functions of the Higgs singlet $F(h)$ and $\mathcal{Y}(h)$ having a priori arbitrarily many parameters.

The corrections received are again functions of the singlet field $h$ and can be encoded in partial differential equations whose solutions are functions of the Higgs singlet and the renormalization scale $\mu$, e.g. $F(h, \mu)$. In order to match with the RGEs given here with the conventional coefficients one can simply perform a Taylor expansion 
on the Higgs functions and match the powers of $h$. Explicitly, the runnings for the different functions are:

$$
\begin{gathered}
\mu \frac{\partial F^{2}}{\partial \mu}-\frac{F F^{\prime}}{16 \pi^{2}} \int d h\left[\frac{\left(g^{\prime}\right)^{2}+3 g^{2}}{2}\left(F^{\prime \prime} F-2\left(F^{\prime}\right)^{2}\right)+4 \operatorname{Tr}\left(\mathcal{Y}^{\prime} \mathcal{Y}^{\dagger \prime}\right)-6 \frac{V^{\prime} F^{\prime \prime} F^{\prime}}{F^{2}}\right] \\
+\frac{2}{16 \pi^{2}}\left[2 \operatorname{Tr}\left(\mathcal{Y} \mathcal{Y}^{\dagger}\right)-\frac{g^{\prime 2}+3 g^{2}}{2} F^{2}+F^{2}\left(F^{\prime 2}-1\right)\left(\frac{g^{\prime 2}}{4}-2 \frac{V^{\prime} F^{\prime}}{F^{3}}\right)-F F^{\prime \prime} V^{\prime \prime}\right]=0 \\
\mu \frac{\partial V}{\partial \mu}-\frac{V^{\prime}}{32 \pi^{2}} \int d h\left[\frac{\left(g^{\prime}\right)^{2}+3 g^{2}}{2}\left(F^{\prime \prime} F-2\left(F^{\prime}\right)^{2}\right)+4 \operatorname{Tr}\left(\mathcal{Y}^{\prime} \mathcal{Y}^{\dagger \prime}\right)-6 \frac{V^{\prime} F^{\prime \prime} F^{\prime}}{F^{2}}\right] \\
-\frac{1}{16 \pi^{2}}\left[\frac{1}{2}\left(V^{\prime \prime}\right)^{2}+\frac{3}{2} \frac{\left(V^{\prime} F^{\prime}\right)^{2}}{F^{2}}-2 \operatorname{Tr}\left(\mathcal{Y}^{\dagger} \mathcal{Y}\right)^{2}-\frac{g^{\prime 2}+3 g^{2}}{4} F^{\prime} V^{\prime}\right. \\
+\frac{3}{2} F^{4} \frac{g^{\prime 4}+2 g^{\prime 2} g^{2}+3 g^{4}}{16}=0, \\
-\mu \frac{\partial \mathcal{Y}}{\partial \mu}+\frac{1}{16 \pi^{2}}\left[g ^ { \prime 2 } \left[Q_{\psi_{L}}^{2} \mathcal{Y}+\mathcal{Y} Q_{\psi_{R}}^{2}-8 Q_{\psi_{L}} \mathcal{Y} Q_{\psi_{R}}-\frac{\mathcal{Y}^{\prime}}{4}\right.\right. \\
\left.+\mathcal{Y}^{\prime}\left(\int d h\left(\frac{F^{\prime \prime} F-2 F^{\prime 2}}{4}\right)-\frac{F^{\prime} F}{4}\right)\right]-8 g_{s}^{2} \mathcal{Y} \\
+3 g^{2} \mathcal{Y}^{\prime}\left(\int d h\left(\frac{F^{\prime \prime} F-2 F^{\prime 2}}{4}\right)-\frac{F^{\prime} F}{4}\right)+\frac{1}{2}\left(\mathcal{Y}^{\prime} \mathcal{Y}^{\dagger \prime} \mathcal{Y}+\mathcal{Y}^{\mathcal{Y}^{\dagger \prime}} \mathcal{Y}^{\prime}\right) \\
+2 \mathcal{Y}^{\prime} \mathcal{Y}^{\dagger} \mathcal{Y}^{\prime}-3 \frac{\tilde{\mathcal{Y}} \tilde{\mathcal{Y}}^{\dagger}}{F^{2}} \mathcal{Y}+\mathcal{Y}^{\prime} \int d h\left(2 \operatorname{Tr}\left(\mathcal{Y}^{\prime} \mathcal{Y}^{\dagger \prime}\right)-3 \frac{V^{\prime} F^{\prime} F^{\prime \prime}}{F^{2}}\right) \\
\left.+V^{\prime \prime} \mathcal{Y}^{\prime \prime}+3 \frac{V^{\prime} F^{\prime}}{F^{3}}\left(\mathcal{Y}^{\prime} F^{\prime} F-\mathcal{Y}\right)\right]=0
\end{gathered}
$$

As mentioned above, we are expanding around the true vacuum so that $V^{\prime}(0)=0$. In order to maintain this condition at the loop level, we have to make sure the RGE for the linear term in $h$ in the potential (the tadpole term) cancels. This term is contained inside the RGE for the potential $V(h)$, in Eq. (7.45). For this we use the renormalization of the Higgs field in Eq. (7.43) choosing the limits of integration so as to cancel the tadpole at one-loop.

We have explicitly checked that the previous RGEs reduce in the SM limit to the corresponding RGEs already computed in the literature [71]. This check can be more directly done by defining $\bar{h}=h+\langle h\rangle$, so the RGEs for the Higgs self-coupling and the Yukawa couplings can be easily compared to those in Ref. [71]. The case of the RGE for the Higgs vev $\langle h\rangle$ is more subtle. Following the procedure described above to impose tadpole cancellation, the RGE for $F^{2}$ gives the running of $v=\langle h\rangle$. If the Higgs redefinition was not chosen to cancel the tadpole term, the definition of $\langle h\rangle$ has to be revised at one-loop. Since at tree level we have $\langle h\rangle^{2}=2 m_{h}^{2} / \lambda$, this means $m_{h}$ has to be promoted to be a running constant as well, so the running for the vev now is given by the running of $2 m_{h}^{2} / \lambda$. 
The renormalization for gauge bosons is just as in the SM and comes from the coefficient of $A_{\mu \nu}^{2}$ in the one-loop effective action for each of the field strengths, see Eq. (7.25). Therefore, the gauge couplings renormalize in the same way as in the SM and the corresponding RGEs are not provided here; but can be consulted also in Ref. [71], noting their conventions (their hypercharge coupling is redefined by a factor $\sqrt{5 / 3})$.

\section{Renormalization of the Sub-leading Lagrangian}

In this case the RGEs are simpler than for the LO Lagrangian, since field renormalization will be a two-loop effect for these operators.

As discussed earlier on, the operator violating the custodial symmetry that should naively be written as LO for dimensions (see Sect. 5.2), is suppressed due to phenomenological reasons. In our notation, this operator reads

$$
\mathcal{L}_{\not}=\frac{C_{\not}}{16 \pi^{2}}\left(d_{\mu} \varphi t_{Y}\right)^{2}
$$

which does arise in our one-loop computation, being controlled by the hypercharge coupling that is inside $t_{Y}$ and the GBs covariant derivative, the Killing vector associated to $U(1)_{Y}$, which is a source of custodial breaking in the SM. The associated RGE is then:

$$
\mu \frac{\partial C_{\not C}}{\partial \mu}-3 F^{2}\left(F^{2}-1\right)=0
$$

and will contribute to the $T$ parameter $₫$ as:

$$
\alpha \delta T=-\frac{3 g^{\prime 2}}{32 \pi^{2}}\left(F^{\prime 2}(0)-1\right) \log \left(\frac{v}{4 \pi f}\right),
$$

where $\alpha=e^{2} /(4 \pi) . \quad \mu=\Lambda=4 \pi f$ is taken to be the scale of new physics behind the pGB Higgs dynamics, which is assumed to respect custodial symmetry. Thus the coefficient is set to vanish at $C_{\not C}(\Lambda)=0$, but is generated when running down in energy till the EW scale $v$.

Now let us list the rest of additional structures found to be required to absorb all the one-loop divergences. They will appear in the Lagrangian as:

$$
\frac{1}{16 \pi^{2}} \sum_{i=1}^{20} C_{i} \mathcal{O}_{i}
$$

Our computation resulted in 20 operators (in addition to $\mathcal{O}_{\not C}$ ) being found to be necessary as counterterms at NLO. Let us first summarize all of them in the following list:

$$
\begin{array}{ll}
\mathcal{O}_{1}=\left(\partial_{\mu} h\right)^{4} / F^{4}, & \mathcal{O}_{5}=\left(d_{\mu} \varphi \partial^{\mu} h\right)^{2} / F^{2}, \\
\mathcal{O}_{2}=\left(\left(d_{\mu} \varphi\right)^{2}\right)^{2}, & \mathcal{O}_{6}=d_{\mu} \varphi^{a} d_{\nu} \varphi_{b} \nabla_{a} t_{B}^{b} A_{\mu \nu}^{B}, \\
\mathcal{O}_{3}=\left(d_{\mu} \varphi d_{\nu} \varphi\right)^{2}, & \mathcal{O}_{7}=\partial^{\mu} h d^{\nu} \varphi \cdot t_{B} A_{\mu \nu}^{B} / F, \\
\mathcal{O}_{4}=\left(\partial_{\nu} h\right)^{2} d_{\mu} \varphi^{2} / F^{2}, & \mathcal{O}_{8}=t_{A} t_{B} A_{\mu \nu}^{B} A^{C, \mu \nu},
\end{array}
$$

`See Ref. [177] and definitions in Sect. 9.2 below. 


$$
\begin{gathered}
\overline{\mathcal{O}}_{9}=\left(\partial_{\mu} h / F\right)^{2}\left(\bar{\psi}_{L} \mathcal{U}\right)^{I} \psi_{R}^{I}, \\
\overline{\mathcal{O}}_{10}=\left(d_{\mu} \varphi\right)^{2}\left(\bar{\psi}_{L} \mathcal{U}\right)^{I} \psi_{R}^{I}, \\
\overline{\mathcal{O}}_{11}=\partial^{\mu} h d_{\mu} \varphi^{a}, \partial_{a}(\bar{\psi} \mathcal{U})^{I} \psi_{R}^{I} / F \\
\mathcal{O}_{12}^{*}=\partial_{\mu} h \bar{\psi}^{I} \gamma_{\mu} \psi_{R}^{I} / F \\
\mathcal{O}_{13}^{*}=\partial_{\mu} h(\bar{\psi} \mathcal{U})^{I} \gamma^{\mu}\left(\mathcal{U}^{\dagger} \psi_{L}\right)^{I} / F \\
\mathcal{O}_{14}=i d_{\mu} \varphi^{a} \bar{\psi}^{I} \gamma^{\mu}\left(\mathcal{U}^{\dagger} \partial_{a} \mathcal{U}\right)_{I J} \psi_{R}^{J}, \\
\mathcal{O}_{15}=i d_{\mu} \varphi^{a}(\bar{\psi} \mathcal{U})^{I} \gamma^{\mu} \overleftrightarrow{\partial}_{a}\left(\mathcal{U}^{\dagger} \psi_{L}\right)^{I} / 2, \\
\mathcal{O}_{16}^{*}=d_{\mu} \varphi^{a} \partial_{a}(\bar{\psi} \mathcal{U})^{I} \gamma^{\mu}\left(\mathcal{U}^{\dagger} \psi_{L}\right)^{I} / 2, \\
\mathcal{O}_{17}=\left(\bar{\psi}_{L} \mathcal{U}\right)^{I} \psi_{R}^{I} \bar{\psi}_{R}^{J}\left(\mathcal{U}^{\dagger} \psi_{L}\right)^{J}, \quad \mathcal{O}_{19}=\bar{\psi}_{R}^{I}\left(\psi_{L} \bar{\psi}_{L} \otimes \mathbb{1}\right)^{I J} \psi_{R}^{J}, \\
\left.\overline{\mathcal{O}}_{18}=\left(\bar{\psi}_{L} \mathcal{U}\right)^{I} \psi_{R}^{I}\left(\bar{\psi}_{L} \mathcal{U}\right)^{J} \psi_{R}^{J}, \quad \overline{\mathcal{O}}_{20} \psi_{R}^{I} \bar{\psi}_{L} \epsilon\right)^{I J} \psi_{R}^{J},
\end{gathered}
$$

where all the barred operators $\left(\overline{\mathcal{O}}_{9-11}, \overline{\mathcal{O}}_{18}\right.$ and $\left.\overline{\mathcal{O}}_{20}\right)$, are added to the action together with their Hermitian conjugate, h.c.; while starred operators are genuinely CPodd.

Again, for simplicity, indices are usually implicit and they should be contracted in order to build invariants under $\mathcal{G}_{S M}$. For instance, the combination $\left(\mathcal{U}^{\dagger} \psi_{L}\right)$ has the $S U(2)_{L}$ indices summed over so it has a flavor index $\alpha=1,2,3$ and a "RH" index $I=u, d, \nu, e:\left(\mathcal{U}^{\dagger} \psi_{L}\right)_{I}^{\alpha}$. Invariance under hypercharge forces some of the fermion flavour indices to be the same, e.g. $\left(\bar{\psi}_{L} \mathcal{U}\right)^{I} \psi_{R}^{J}$ only is $U(1)_{Y}$-invariant for $I=J$. As it will be shown below, in the explicit expressions for the RGEs, the coefficients that contain Yukawa couplings show the corresponding index $I$ indicating the type of $\mathrm{RH}$ fermion and also two flavor indices $Y_{I}^{\alpha \beta}$.

Inside operator $\mathcal{O}_{19}$ the $S U(2)_{L}$ indices are contracted in such a way that the $\mathrm{LH}$ fermions explicitly appear as $\left\{\left(q \bar{q}_{L} \mathbb{1}, q \bar{\ell}_{L} \mathbb{1}\right),\left(\ell \bar{q}_{L} \mathbb{1}, \ell \bar{\ell}_{L} \mathbb{1}\right)\right\}$. On the other hand, in $\overline{\mathcal{O}}_{20}$ it is understood that $\left[\bar{\psi}_{L} \varepsilon() \bar{\psi}_{L} \epsilon\right]=\left[\bar{\psi}_{L} \varepsilon() \bar{\psi}_{L} \otimes \epsilon\right]$ where we insert $\psi_{R}^{I}$ in () and $\varepsilon$ is the antisymmetric tensor with $S U(2)_{L}$ implicit indices.

Some pieces from these structures do violate custodial symmetry. For instance, $\mathcal{O}_{13}^{*}$ could be decomposed into a custodial preserving and custodial breaking piece, schematically:

$$
\begin{aligned}
\mathcal{O}_{13}^{*}=C_{13}(Y) & \frac{\partial_{\mu} h}{F}(\bar{\psi} \mathcal{U}) \gamma^{\mu}\left(\mathcal{U}^{\dagger} \psi_{L}\right) \\
& \rightarrow \hat{C}(Y) \frac{\partial_{\mu} h}{F} \bar{\psi} \gamma^{\mu} \psi_{L}+\tilde{C}(Y) \frac{\partial_{\mu} h}{F} \bar{\psi} \gamma^{\mu}\left(\mathcal{U} \tau_{3} \mathcal{U}^{\dagger}\right) \psi_{L},
\end{aligned}
$$

where the custodial breaking piece contains $\mathcal{U} \tau_{3} \mathcal{U}^{\dagger}$, see Eq. (5.13).

Finally, we do not obtain any dipole or field strength to the cube structures. Holomorphy and helicity sum rules could help to make sense of this absence, as in Refs. [178, 179].

With respect to Refs. $[28,148,149]$, where only scalars are taken to run in the loops, we obtain an extra set of structures, made of scalar and fermion currents, as shown in Eq. (7.53). 


\section{Renormalization Group Equations}

The corresponding RGEs for the different groups of operators in Eqs. (7.51)-(7.54) are:

Bosonic operators.

$$
\begin{aligned}
\mu \frac{d C_{1}}{d \mu} & =-\frac{3}{2}\left(F F^{\prime \prime}\right)^{2}, & \mu \frac{d C_{5}}{d \mu} & =-\frac{4}{3}\left(F F^{\prime \prime}\right)^{2}, \\
\mu \frac{d C_{2}}{d \mu} & =-\frac{1}{2}\left(F F^{\prime \prime}\right)^{2}-\frac{1}{3}\left(F^{2}-1\right)^{2}, & \mu \frac{d C_{6}}{d \mu} & =\frac{1}{3}\left(F^{2}-1\right), \\
\mu \frac{d C_{3}}{d \mu} & =-\frac{2}{3}\left(F^{\prime 2}-1\right)^{2}, & \mu \frac{d C_{7}}{d \mu} & =\frac{2}{3} F^{\prime} F F^{\prime \prime}, \\
\mu \frac{d C_{4}}{d \mu} & =\frac{\left(F F^{\prime \prime}\right)^{2}}{3}-2\left(F^{\prime 2}-1\right) F F^{\prime \prime}, & \mu \frac{d C_{8}}{d \mu} & =\frac{1}{6}\left(F^{2}-1\right),
\end{aligned}
$$

\section{Boson-Yukawa operators.}

$$
\begin{aligned}
\mu \frac{d C_{9}}{d \mu} & =3 F^{\prime \prime}\left(F^{\prime} Y_{I}^{\prime}-\frac{Y_{I}}{F}\right), \\
\mu \frac{d C_{10}}{d \mu} & =F F^{\prime \prime} Y_{I}^{\prime \prime}+2 \frac{F^{2}-1}{F}\left(F^{\prime} Y_{I}^{\prime}-\frac{Y_{I}}{F}\right), \\
\mu \frac{d C_{11}}{d \mu} & =-2 F F^{\prime \prime}\left(\frac{Y_{I}}{F}\right)^{\prime},
\end{aligned}
$$

Scalar current - fermion current operators.

$$
\begin{aligned}
\mu \frac{d C_{12}}{d \mu} & =-\frac{i F}{2}\left[Y_{I}^{\prime \dagger} Y_{I}^{\prime \prime}+3 \frac{Y_{I}^{\dagger}}{F}\left(\frac{Y_{I}}{F}\right)^{\prime}\right]+h . c . \\
\mu \frac{d C_{13}}{d \mu} & =-\frac{i F}{2}\left[Y_{I}^{\prime} Y_{I}^{\prime \prime \dagger}+\frac{Y_{I}}{F}\left(\frac{Y_{I}^{\dagger}}{F}\right)^{\prime}+2 \frac{Y_{I}}{F}\left(\frac{\tilde{Y}_{I}^{\dagger}}{F}\right)^{\prime}\right]+h . c . \\
\mu \frac{d C_{14}}{d \mu} & =\frac{F^{\prime}}{F}\left(Y_{I}^{\dagger} Y_{J}\right)^{\prime}-Y_{I}^{\prime \dagger} Y_{J}^{\prime}-\frac{Y_{I}^{\dagger} Y_{J}}{F^{2}} \\
\mu \frac{d C_{15}}{d \mu} & =\left(\frac{F^{\prime}}{F}\left(Y_{I} Y_{I}^{\dagger}\right)^{\prime}-Y_{I}^{\prime} Y_{I}^{\prime \dagger}-\frac{Y_{I} Y_{I}^{\dagger}}{F^{2}}\right) \\
\mu \frac{d C_{16}}{d \mu} & =-\frac{i}{2}\left(\frac{F^{\prime}}{F} Y_{I} Y_{I}^{\prime \dagger}-\frac{F^{\prime}}{F}\left(\tilde{Y} \tilde{Y}^{\prime \dagger}\right)_{I}\right)+\text { h.c., }
\end{aligned}
$$




\section{Four-fermion operators.}

$$
\begin{gathered}
\mu \frac{d C_{17}}{d \mu}=2\left(\frac{Y_{I}}{F}\right)^{\prime}\left(\frac{Y_{J}^{\dagger}}{F}\right)^{\prime}-Y_{I}^{\prime \prime} Y_{J}^{\prime \prime \dagger}-\frac{3}{F^{2}}\left(F^{\prime} Y_{I}^{\prime}-\frac{Y_{I}}{F}\right)\left(F^{\prime} Y_{J}^{\dagger}-\frac{Y_{J}^{\dagger}}{F}\right) \\
\mu \frac{d C_{18}}{d \mu}=\left(\frac{Y_{I}}{F}\right)^{\prime}\left(\frac{Y_{J}}{F}\right)^{\prime}-\frac{1}{2} Y_{I}^{\prime \prime} Y_{J}^{\prime \prime}-\frac{3}{2 F^{2}}\left(F^{\prime} Y_{I}^{\prime}-\frac{Y_{I}}{F}\right)\left(F^{\prime} Y_{J}^{\prime}-\frac{Y_{J}}{F}\right) \\
\mu \frac{d C_{19}}{d \mu}=-4\left(\frac{Y_{I}^{\dagger}}{F}\right)^{\prime}\left(\frac{Y_{J}}{F}\right)^{\prime} \\
\mu \frac{d C_{20}}{d \mu}=-2\left(\frac{Y_{I}}{F}\right)^{\prime}\left(\frac{Y_{J}}{F}\right)^{\prime}
\end{gathered}
$$

As explained above, $Y_{I}$ for $I=u, d, \nu, e$ are the different types of Yukawa couplings with two flavor indices. Eq. (7.58) shows that the coefficients of the CP-odd operators $\left(\mathcal{O}_{12}^{*}, \mathcal{O}_{13}^{*}, \mathcal{O}_{16}^{*}\right)$ are proportional to the imaginary part of the product of different Yukawa terms. The results shown here are in agreement with computations considering only scalars in the loops, Refs. [148,149], which also agree with Ref. [28] and our previous results for the scalar sector of Chapter 6.

The phenomenology associated to the one-loop HEFT can be studied upon these results, which is beyond the scope of this work. We have presented the renormalization of the LO custodial-invariant HEFT Lagrangian of Eq. (7.36) and have determined the set of operators required at NLO by renormalization with the corresponding RGEs for their coefficients. With this, the general discussion on the Higgs effective field theory in this thesis is concluded. We will however come back to the connection with EFTs when the benchmark Lagrangian from an $S O(5) / S O(4)$ model for a pGB Higgs will be determined in Chapter 10. Also, in the last chapter, we will develop the basis of first-order baryon number violating operators within the HEFT. 


\section{Part III}

Linear sigma model for a pseudo-Goldstone Higgs 



\section{Chapter 8}

\section{Presentation of the model}

In order to explore a possible dynamical nature for the Higgs field we introduce in this chapter a complete renormalizable Lagrangian based on the minimal $S O(5)$ linear $\sigma$ model with the symmetry softly broken to $\mathrm{SO}(4)$; including gauge bosons and fermions, as it is presented in [180]. In the next chapter, we discuss the phenomenological implications and constraints from precision observables and the impact on LHC data.

The model is inspired by the composite Higgs framework, which has been explained in Sect. 4.3; implemented in a minimal $S O(5) \rightarrow S O(4)$ breaking scheme [30]. See the schematics in Fig. 8.1. The high-energy global $S O(5)$ symmetry is broken spontaneously to $S O(4)$, resulting in four massless Goldstone bosons with characteristic scale $f$; analogous to the $f_{\pi}$ pion decay constant in QCD. The four components of the Higgs multiplet then share a common GB origin; while there is an extra scalar, $\sigma$, singlet under $S O(4)$, which acquires a mass already from the spontaneous symmetry breaking.

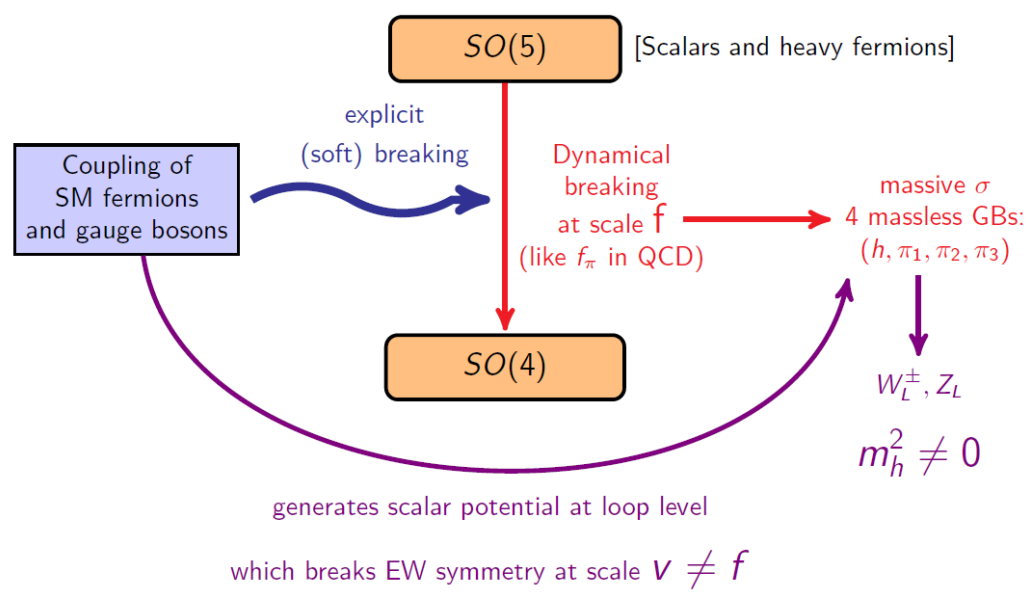

Figure 8.1: Schematics of the $S O(5) \rightarrow S O(4)$ model 
Furthermore, $S O(5)$ is explicitly broken by the coupling of the exotic heavy representations to the SM fermions and by the couplings with the EW gauge bosons in the covariant derivatives. This induces at one-loop a potential for the $h$ field with a non-trivial minimum, providing a mass for $h$ so it becomes a pseudo-Goldstone boson and breaking the SM electroweak symmetry at a scale $v \neq f$.

Most of the literature on composite Higgs uses an effective non-linear approach to study the models; see for instance [31,32,101,102,109,181-184]. Instead, we study here a renormalizable model, a particular UV completion of those. A former analysis in this direction can be found in [34].

By varying the mass of the extra scalar field, $\sigma$, it is possible to sweep from the linear, weakly coupled regime (light $\sigma$ particle) to the non-linear one $\left(m_{\sigma} \rightarrow \infty\right)$, where one should fall onto the standard effective approach. This limit, and the dominant effects from the linear completion will be explored in Chapter 10. As stated in the motivation for this thesis, another advantage of this model is that it might be considered either as a renormalizable UV completion of some deeper dynamics; or it can be regarded as a UV-complete model describing elementary fields.

The choice of the minimal bosonic sector is clear and seems rather model-independent (it could also be used to describe scenarios containing a $S O(5) / S O(4)$ ). However, there are several possible choices for the fermionic sector, an issue that will be tackled in an effective way in the analysis of Sect. 10.1.

The option explored in this paper assumes heavy fermions in vectorial representations of $S O(5)$; as is explained in Sect. 8.2. Direct linear couplings between the exotic fermions and the SM ones will be the primordial source of $S O(5)$ explicit breaking. The Higgs particle has tree-level couplings only with the exotic fermionic sector, via $S O(5)$-invariant Yukawa couplings; while light fermion masses are generated through a "partial compositeness" [35] mechanism. It will be finally discussed how the induced Coleman-Weinberg potential requires soft-breaking terms to be included in the scalar potential from the contributions of heavy fermions.

The complete Lagrangian can then be written as the sum of three terms describing respectively the pure gauge, scalar and fermionic sectors,

$$
\mathcal{L}=\mathcal{L}_{\text {gauge }}+\mathcal{L}_{\text {scalar }}+\mathcal{L}_{\text {fermion }}
$$

where $\mathcal{L}_{\text {gauge }}$ is the SM gauge kinetic terms. This next section discusses in detail the scalar sector and its interactions; while the fermionic sector is left for the final part of the chapter.

\subsection{The $S O(5) / S O(4)$ scalar sector}

The linear $\sigma$ model corresponding to an $S O(5)$ symmetry spontaneously broken to $S O(4)$ is inspired by the linear sigma model of QCD (which features a spontaneous breaking isomorphic to $S O(4) \rightarrow S O(3)$; see Sect. 2.2.2), with potential of the form:

$$
V=\lambda\left(\Phi^{T} \Phi-f^{2}\right)^{2},
$$


where we have rewritten Eq. (2.26) with a real scalar field $\Phi$, transforming under the 4 of $S O(4)$.

Since we want to have one more GB, let us now enlarge the scalar field to a $\phi$ that has one more component, so it transforms in the fundamental (5) representation of $S O(5)$. This implies we have five real degrees of freedom: three of them to be ultimately associated with the longitudinal components of the SM gauge bosons $\left(\boldsymbol{\pi}=\left(\pi_{1}, \pi_{2}, \pi_{3}\right)\right)$; one to correspond to the Higgs particle $h$ and the remaining one to be the scalar denoted by $\sigma$, singlet under $S O(4)$ :

$$
\phi=\left(\pi_{1}, \pi_{2}, \pi_{3}, h, \sigma\right)^{T} \stackrel{\text { u.g. }}{\rightarrow} \quad(0,0,0, h, \sigma)^{T} .
$$

where u.g. stands for unitary gauge $(\boldsymbol{\pi}=0)$. For simplicity, from now on all expressions will be shown in this gauge. The scalar Lagrangian then reads:

$$
\mathcal{L}_{\mathrm{s}}=\frac{1}{2}\left(D_{\mu} \phi\right)^{T}\left(D^{\mu} \phi\right)-V(\phi)
$$

where $D_{\mu}$ is the $S U(2)_{L} \times U(1)_{Y}$ covariant derivative, given by

$$
D_{\mu} \phi=\left(\partial_{\mu}+i g \Sigma_{L}^{i} W_{\mu}^{i}+i g^{\prime} \Sigma_{R}^{3} B_{\mu}\right) \phi
$$

and $\Sigma_{L}^{i}$ and $\Sigma_{R}^{i}$ denote respectively the generators of the $S U(2)_{L}$ and $S U(2)_{R}$ subgroups of the custodial $S O(4)$ group contained in $S O(5)$.

An equivalent notation for $\phi$ can be written in terms of the SM Higgs doublet, $H$, and its charge conjugate, $\tilde{H}$ :

$$
\hat{\phi}=\frac{1}{\sqrt{2}}\left(\tilde{H}^{T}, H^{T}, \sqrt{2} \sigma\right)^{T}
$$

with

$$
H=\left(\begin{array}{l}
H^{u} \\
H^{d}
\end{array}\right) \quad \stackrel{\text { u.g. }}{\rightarrow} \quad \frac{1}{\sqrt{2}}\left(\begin{array}{l}
0 \\
h
\end{array}\right), \quad \tilde{H} \equiv i \sigma_{2} H^{*}=\left(\begin{array}{l}
\tilde{H}^{u} \\
\tilde{H}^{d}
\end{array}\right) \quad \stackrel{\text { u.g. }}{\rightarrow} \quad \frac{1}{\sqrt{2}}\left(\begin{array}{l}
h \\
0
\end{array}\right) .
$$

The hat on $\phi$ is to distinguish from the scalar field in the real notation above. The last component, $\sigma$, is an $S U(2)_{L}$ and $S U(2)_{R}$ singlet. The relation between the real and the complex notation is given by

$$
\phi=\frac{1}{\sqrt{2}}\left(-i\left(H^{u}+\tilde{H}^{d}\right), H^{u}-\tilde{H}^{d}, i\left(H^{d}-\tilde{H}^{u}\right), H^{d}+\tilde{H}^{u}, \sqrt{2} \sigma\right)^{T} .
$$

\section{The scalar potential}

We are interested in including explicit terms modeling the explicit breaking of $S O(5)$. In the unitary gauge, the most general renormalizable potential with broken $S O(5)$; but preserving $S O(4)$ reads:

$$
\begin{aligned}
V(h, \sigma)=\lambda\left(\sigma^{2}+h^{2}-f^{2}\right)^{2}+\alpha f^{3} \sigma-f^{2} \beta h^{2} \\
+a_{1} f \sigma h^{2}+a_{2} \sigma^{2} h^{2}+a_{3} f \sigma^{3}+a_{4} h^{4} .
\end{aligned}
$$


where the expression in a general gauge can be recovered simply by doing the substitution $h^{2} \rightarrow h^{2}+\pi^{2}$. We have chosen to get rid of $\sigma^{2}$ and $\sigma^{4}$ terms; since they can be absorbed in a redefinition of other parameters; which results in only eight parameters.

However, in order to be even more minimal, we will keep the only terms that are strictly necessary for a soft explicit breaking. They are the $\alpha$ and $\beta$ terms; since they will be needed as counterterms to absorb divergences generated by one-loop Coleman-Weinberg contributions to the Lagrangian from the heavy fermions, as it is shown in Sect. 8.3. Therefore, the final expression for the Lagrangian is simply*

$$
\begin{aligned}
\mathcal{L}_{s}= & \frac{1}{2} \partial_{\mu} \sigma \partial^{\mu} \sigma+\frac{1}{2} \partial_{\mu} h \partial^{\mu} h-V(h, \sigma) \\
& V(h, \sigma)=\lambda\left(\sigma^{2}+h^{2}-f^{2}\right)^{2}+\alpha f^{3} \sigma-f^{2} \beta h^{2} .
\end{aligned}
$$

This two extra $\alpha$ and $\beta$ terms have also been considered in Ref. [34]; where also a simple analysis was performed. The resulting scalar Lagrangian depends on four parameters.

EWSB requires both scalars $h, \sigma$ to acquire a non-vanishing vev, respectively dubbed as $v$ and $v_{\sigma}$ below, as for $v \neq 0$ the $S O(4)$ global group and the EW group are spontaneously broken. Therefore, for $\alpha, \beta \neq 0$ and assuming $v \neq 0$, it results that the potential has a minimum at

$$
v_{\sigma}^{2} \equiv\langle\sigma\rangle^{2}=f^{2} \frac{\alpha^{2}}{4 \beta^{2}} \quad, \quad v^{2} \equiv\langle h\rangle^{2}=f^{2}\left(1-\frac{\alpha^{2}}{4 \beta^{2}}+\frac{\beta}{2 \lambda}\right),
$$

satisfying the condition

$$
v^{2}+v_{\sigma}^{2}=f^{2}(1+\beta / 2 \lambda),
$$

The focus of this paper is set on the interpretation of the Higgs particle as a PNGB, which requires $f^{2}>0$ as well as $|v|<\left|v_{\sigma}\right|$; see Sect. 8.1.4 for a discussion on the scalar parameter space. For $f^{2}>0, v^{2}>0$ and $|v|<\left|v_{\sigma}\right|$ we have the following conditions:

$$
\begin{aligned}
& \alpha^{2}<4 \beta^{2}\left(1+\frac{\beta}{2 \lambda}\right), \\
& 2 \beta^{2}\left(1+\frac{\beta}{2 \lambda}\right)<\alpha^{2},
\end{aligned}
$$

which for $|\beta| \ll \lambda$ would indicate $2 \beta^{2} \lesssim \alpha^{2} \lesssim 4 \beta^{2}$. Moreover, in order to get $v^{2} \ll f^{2}$, Eq. (8.11) requires a fine-tuning such that the ratio $\alpha / 2 \beta$ has to be close to one.

Expanding now the $\sigma$ and $h$ fields around their minima, $h \equiv \hat{h}+v$ and $\sigma \equiv \hat{\sigma}+v_{\sigma}$, and diagonalizing the scalar mass matrix, the mass eigenstates are given by

$$
\text { Light }=\hat{h} \cos \gamma-\hat{\sigma} \sin \gamma, \quad \text { Heavy }=\hat{\sigma} \cos \gamma+\hat{h} \sin \gamma .
$$

*Full renormalizability of the theory requires, in general, the presence of all gauge invariant operators of dimension equal to or smaller than four. At two or more loops, the renormalization procedure may thus require to include further symmetry breaking terms beyond those considered; we will assume that their finite contributions will be weighted by comparatively negligible coefficients and can be safely omitted in our analysis. 
It will be later shown that, for a pGB Higgs particle the less fine-tuned regions in parameter space correspond to the case in which the light state is the Higgs and the heavy state is the sigma particle. Therefore, in the phenomenological discussion of the model we will use $h$ and $\sigma$ again for simplicity and take Light $=h$ and Heavy $=\sigma$.

The mixing angle in Eq. (8.15) is given by

$$
\tan 2 \gamma=\frac{4 v v_{\sigma}}{3 v_{\sigma}^{2}-v^{2}-f^{2}}
$$

Note that it should remain in the interval $\gamma \in[-\pi / 4, \pi / 4]$ in order not to interchange the roles of the heavy and light mass eigenstates.

The mass eigenvalues are given by

$$
m_{\text {heavy, light }}^{2}=4 \lambda f^{2}\left\{\left(1+\frac{3}{4} \frac{\beta}{\lambda}\right) \pm\left[1+\frac{\beta}{2 \lambda}\left(1+\frac{\alpha^{2}}{2 \beta^{2}}+\frac{\beta}{8 \lambda}\right)\right]^{1 / 2}\right\}
$$

where the plus sign refers to the heavier eigenstate. For $f^{2}>0$, the squared masses are positive if the following two conditions are satisfied:

$$
3 \beta+4 \lambda>0, \quad 2 \beta^{2}+4 \beta \lambda-\alpha^{2} \lambda / \beta>0,
$$

where the second constraint is equivalent to Eq. (8.13). It follows that ${ }^{\dagger} \beta>0$.

Assuming the $S O(5)$ explicit breaking to be small: $|\beta| / 4 \lambda \ll 1$ the masses of the heavy and light eigenstates read

$$
\begin{aligned}
m_{\text {heavy }}^{2} & =8 \lambda f^{2}+2 \beta\left(3 f^{2}-v^{2}\right)+O\left(\frac{\beta}{4 \lambda}\right), \\
m_{\text {light }}^{2} & =2 \beta v^{2}+O\left(\frac{\beta}{4 \lambda}\right) .
\end{aligned}
$$

The physical scalars thus correspond to a "light" state with mass $O(\sqrt{\beta} v)$ and a "heavy" state with mass $O(\sqrt{\lambda} f)$.

As a consequence of the first expression, the scalar quartic coupling $\lambda$ can be conventionally traded by the sigma mass, usually keeping only the dominant term: $m_{\sigma}^{2} \simeq 8 \lambda f^{2}$ The non-linear model would be recovered in the limit $m_{\sigma} \gg f$; i.e. $\lambda \gg 1$.

Notice also that for $m_{h}<m_{\sigma}$ in the regime of small soft $S O(5)$ breaking the mass of the Higgs is controlled by $\beta$, a different parameter than the quartic self-coupling, $\lambda$. This is consistent with the pGB nature of the Higgs boson whose mass should now appear protected from growing in the strong interacting regime of the theory (given by $\lambda$ ) in which instead the $\sigma$ mass would increase. In other words, we have replaced the hierarchy problem for the Higgs particle mass by a sensitivity of the $\sigma$ particle to heavier scales. In this way the $\sigma$ mass represents generically the heavy UV completion. Finally, note that the expression for $m_{h}$ shows that the value of the $\beta$ parameter for small $\beta / 4 \lambda$ is expected to be around $\beta \sim m_{h}^{2} / 2 v^{2} \sim 0.13$.

\footnotetext{
${ }^{\dagger}$ For $f^{2}<0$, both inequalities in Eq. (8.18) are reverted (and so is also the inequality in Eq. (8.13)); as a consequence, in that case we have $\beta<0$.
} 


\section{Scalar-gauge boson couplings}

In the unitary gauge, the kinetic scalar Lagrangian written in terms or the unrotated fields has the following expression:

$$
\mathcal{L}_{s, k i n}=\frac{1}{2}\left(\partial_{\mu} \hat{\sigma}\right)^{2}+\frac{1}{2}\left(\partial_{\mu} \hat{h}\right)^{2}+\frac{g^{2}}{4}(\hat{h}+v)^{2} W_{\mu}^{+} W^{\mu-}+\frac{\left(g^{2}+g^{\prime 2}\right)}{8}(\hat{h}+v)^{2} Z_{\mu} Z^{\mu},
$$

which justifies the previous identification of the Higgs vev $v$ with the EW scale defined from the $W$ mass

$$
v=246 \mathrm{GeV} .
$$

In terms of the physical $h, \sigma$ fields $\left(m_{h}<m_{\sigma}\right){ }^{\ddagger}$, the Lagrangian reads

$$
\begin{aligned}
\mathcal{L}_{s} & =\frac{1}{2}\left(\partial_{\mu} \sigma\right)^{2}+\frac{1}{2}\left(\partial_{\mu} h\right)^{2}-\frac{1}{2} m_{\sigma}^{2} \sigma^{2}-\frac{1}{2} m_{h}^{2} h^{2}-\lambda\left(h^{2}+2 h \sigma+\sigma^{2}\right)^{2}- \\
& -4 \lambda\left(v \cos \gamma-v_{\sigma} \sin \gamma\right)\left(h^{3}+h \sigma^{2}\right)-4 \lambda\left(v \sin \gamma+v_{\sigma} \cos \gamma\right)\left(\sigma^{3}+h^{2} \sigma\right)+ \\
& +\left(1+\frac{h}{v} \cos \gamma+\frac{\sigma}{v} \sin \gamma\right)^{2}\left(M_{W}^{2} W_{\mu}^{+} W^{\mu-}+\frac{1}{2} M_{Z}^{2} Z_{\mu} Z^{\mu}\right)
\end{aligned}
$$

As a consequence of the rotation to the mass basis, the physical Higgs couplings are thus seen to be suppressed by a $\cos \gamma$ factor with respect to the SM ones. Besides, the physical $\sigma$ particle does also couple to the EW sector due to the mixing with the original Higgs; with couplings suppressed by $\sin \gamma$ instead. This fact will yield interesting phenomenology, as it will be discussed in the next chapter. The SM limit is recovered when the $\sigma$ field is decoupled from the spectrum, with $\cos \gamma=1$.

\section{Renormalization and scalar tree-level decays}

The four independent parameters of the scalar Lagrangian, $f, \lambda, \alpha$ and $\beta$, can be expressed in terms of the following observables:

$$
G_{F} \equiv\left(\sqrt{2} v^{2}\right)^{-1}, \quad m_{h}, \quad m_{\sigma}, \quad \sin \gamma,
$$

with the Fermi constant $G_{F}$ as measured from muon decay $m_{h}$ from the Higgs pole mass, and $m_{\sigma}$ could be determined from future measurements of the $\sigma$ mass. The mixing is given by $\sin \gamma$; which could be measured from either deviations of the Higgs couplings, as in $\kappa_{V}$ or from the $\sigma$ line shape obtained from its decay into four leptons for $m_{\sigma} \geq 300 \mathrm{GeV}$, analogous to the case of a heavy SM Higgs boson ${ }^{\S}$.

To extend the renormalization scheme to the gauge sector, we choose two extra observables: the mass of the $Z$ boson and the fine structure constant,

$$
M_{Z}, \quad \alpha_{e m}=\frac{e^{2}}{4 \pi},
$$

\footnotetext{
${ }^{\ddagger}$ If $m_{h}>m_{\sigma}$ the mixing dependence would correspond to the interchange $\cos \gamma \leftrightarrow \sin \gamma$ in Eq. (8.21)

$\S$ For a lighter $\sigma$, the decay width becomes too narrow (maybe even below the experimental resolution) and more ingenious procedures would be required to determine the scalar mixing strength, such as for instance on-shell to off-shell cross section measurements [185].
} 
with $M_{Z}$ and $\alpha_{e m}$ determined from Z-pole mass measurements and from Thompson scattering, respectively [41]. In our model, the relation between the gauge boson masses is the same as the SM one:

$$
M_{W}=\cos \theta_{W} M_{Z},
$$

where the weak angle is given at tree-level by

$$
\sin ^{2} \theta_{W}=\frac{1}{2}\left(1-\sqrt{1-\frac{4 \pi \alpha_{e m}}{\sqrt{2} G_{F} M_{Z}^{2}}}\right) .
$$

Finally, the relevant tree-level branching ratios for the heavy and light scalar boson decays into the SM EW gauge bosons can be computed straightforwardly:

$$
\begin{aligned}
\Gamma\left(h \rightarrow W W^{*}\right) & =\Gamma_{\mathrm{SM}}\left(h \rightarrow W W^{*}\right) \cos ^{2} \gamma, \\
\Gamma\left(h \rightarrow Z Z^{*}\right) & =\Gamma_{\mathrm{SM}}\left(h \rightarrow Z Z^{*}\right) \cos ^{2} \gamma, \\
\Gamma\left(\sigma \rightarrow W^{+} W^{-}\right) & =\frac{\sqrt{2} G_{F}}{16 \pi} m_{\sigma}^{3} \sin ^{2} \gamma\left[1+\mathcal{O}\left(\frac{M_{W}^{2}}{m_{\sigma}^{2}}\right)\right], \\
\Gamma(\sigma \rightarrow Z Z) & =\frac{\sqrt{2} G_{F}}{32 \pi} m_{\sigma}^{3} \sin ^{2} \gamma\left[1+\mathcal{O}\left(\frac{M_{Z}^{2}}{m_{\sigma}^{2}}\right)\right],
\end{aligned}
$$

where the SM widths can be found for instance in Ref. [186]. Furthermore, the decay of the heavy scalar $\sigma$ into two Higgs particles is:

$$
\Gamma(\sigma \rightarrow h h)=\frac{\sqrt{2} G_{F}}{32 \pi} m_{\sigma}^{3} \sin ^{2} \gamma\left[1+\mathcal{O}\left(\frac{m_{h}^{2}}{m_{\sigma}^{2}}\right)\right] .
$$

In conclusion, the $\sigma$ partial widths into EW gauge bosons and the Higgs will dominate the total $\sigma$ width unless the mixing is unnaturally tiny. Therefore

$$
\frac{\Gamma_{\sigma}}{m_{\sigma}} \simeq \frac{m_{\sigma}^{2} \sin ^{2} \gamma}{8 v^{2}}
$$

and thus the measurement of the line shape of the $\sigma$ seems feasible only for $m_{\sigma}$ above the EW breaking scale (assuming non-negligible mixing). In that regime, the value of $\sin \gamma$ can be inferred from the line shape and all other observables in Eq. (8.26) can then be predicted in terms of the physical parameters defining our renormalization scheme. Other bosonic decay channels requiring one-loop amplitudes (decays into gluons and photons) will be discussed in Sects. 9.3 and 9.4.

\section{Scalar parameter space}

We can now cast the any parameter in terms of those physical quantities. For instance, using Eqs. (8.11), (8.16) and (8.17), the exact expressions for the $h$ and $\sigma$ vevs are:

$$
\begin{aligned}
v & =\left(\sqrt{2} G_{F}\right)^{-1 / 2} \\
v_{\sigma} & =\frac{v \sin (2 \gamma)\left(m_{\sigma}^{2}-m_{h}^{2}\right)}{m_{\sigma}^{2}+m_{h}^{2}-\left(m_{\sigma}^{2}-m_{h}^{2}\right) \cos (2 \gamma)} .
\end{aligned}
$$


And the expressions for the parameters of the scalar potential (some of the parameters have been chosen to appear in combinations due to its convenience and simplicity of the resulting expression):

$$
\begin{aligned}
\lambda & =\frac{\sin ^{2} \gamma m_{\sigma}^{2}}{8 v^{2}}\left(1+\cot ^{2} \gamma \frac{m_{h}^{2}}{m_{\sigma}^{2}}\right), \\
\frac{\beta}{4 \lambda} & =\frac{m_{h}^{2} m_{\sigma}^{2}}{\sin ^{2} \gamma m_{\sigma}^{4}+\cos ^{2} \gamma m_{h}^{4}-2 m_{h}^{2} m_{\sigma}^{2}}, \\
\frac{\alpha^{2}}{4 \beta^{2}} & =\frac{\sin ^{2}(2 \gamma)\left(m_{\sigma}^{2}-m_{h}^{2}\right)^{2}}{4\left(\sin ^{2} \gamma m_{\sigma}^{4}+\cos ^{2} \gamma m_{h}^{4}-2 m_{h}^{2} m_{\sigma}^{2}\right)}, \\
f^{2} & =\frac{v^{2}\left(\sin ^{2} \gamma m_{\sigma}^{4}+\cos ^{2} \gamma m_{h}^{4}-2 m_{h}^{2} m_{\sigma}^{2}\right)}{\left(\sin ^{2} \gamma m_{\sigma}^{2}+\cos ^{2} \gamma m_{h}^{2}\right)^{2}} .
\end{aligned}
$$

The last expression shows that $\sin ^{2} \gamma$ does not coincide with the parameter $\xi=v^{2} / f^{2}$ commonly used in the literature about composite Higgs models, except in the limit $m_{\sigma} \gg m_{h}$ (or more precisely $\beta / 4 \lambda \ll 1$ and $v^{2} \ll f^{2}$ ), where for sizable $\sin \gamma$ the last equation above leads to

$$
\sin ^{2} \gamma_{m_{\sigma} / m_{h} \gg 1}^{\longrightarrow} \frac{v^{2}}{f^{2}}+4 \frac{m_{h}^{2}}{m_{\sigma}^{2}} .
$$

With the aid of this set of exact relations it is particularly instructive to fix the Higgs vev and mass to their known experimental values and plot the parameter space in terms as $\sin ^{2} \gamma$ versus $m_{\sigma}$, as in Fig. 8.2. The light red region corresponds to $f^{2}<0$. In this case the minimum of the potential is at the origin and thus no spontaneous breaking of $S O(5)$ occurs; so no scalar is a GB. The red borders correspond to $f^{2}=0$. On the other hand, in the light brown region, $v_{\sigma}<v$; whereas in the white regions $v<v_{\sigma}$, where the vevs are defined with respect to the unrotated fields. The regions can be connected continuously to the limit where the smaller vev is zero and the field is a true GB. Thus the Higgs will behave mostly as the pGB in the lower-right and upper-left corners (see below). In consequence, it turns out that arbitrary values of $m_{\sigma}$ and $\sin ^{2} \gamma$ are not allowed if we insist on interpreting the Higgs boson as the pseudo-Goldstone boson of a spontaneous $S O(5)$ breaking.

It is also interesting to discuss the plot regarding the scalar masses $\left(m_{h}=125\right.$ $\mathrm{GeV})$.

- In the case of $m_{h}<m_{\sigma}$ (right of the figure), the physical Higgs particle corresponds to the light state, while the physical sigma is the heavy one:

$$
\begin{aligned}
\sigma_{\text {phys }} & =\hat{\sigma} \cos \gamma+\hat{h} \sin \gamma, \\
h_{\text {phys }} & =\hat{h} \cos \gamma-\hat{\sigma} \sin \gamma,
\end{aligned}
$$

The physical Higgs couplings to SM particles are thus suppressed by $\cos \gamma$ with respect to SM values; see for instance Eq. (8.21). In this case it will be shown in Sect. 9.1 that present LHC Higgs data only allow for values $\sin ^{2} \gamma<0.18$

『For $f^{2}<0, \alpha$ would have to be purely imaginary because of hermiticity. 


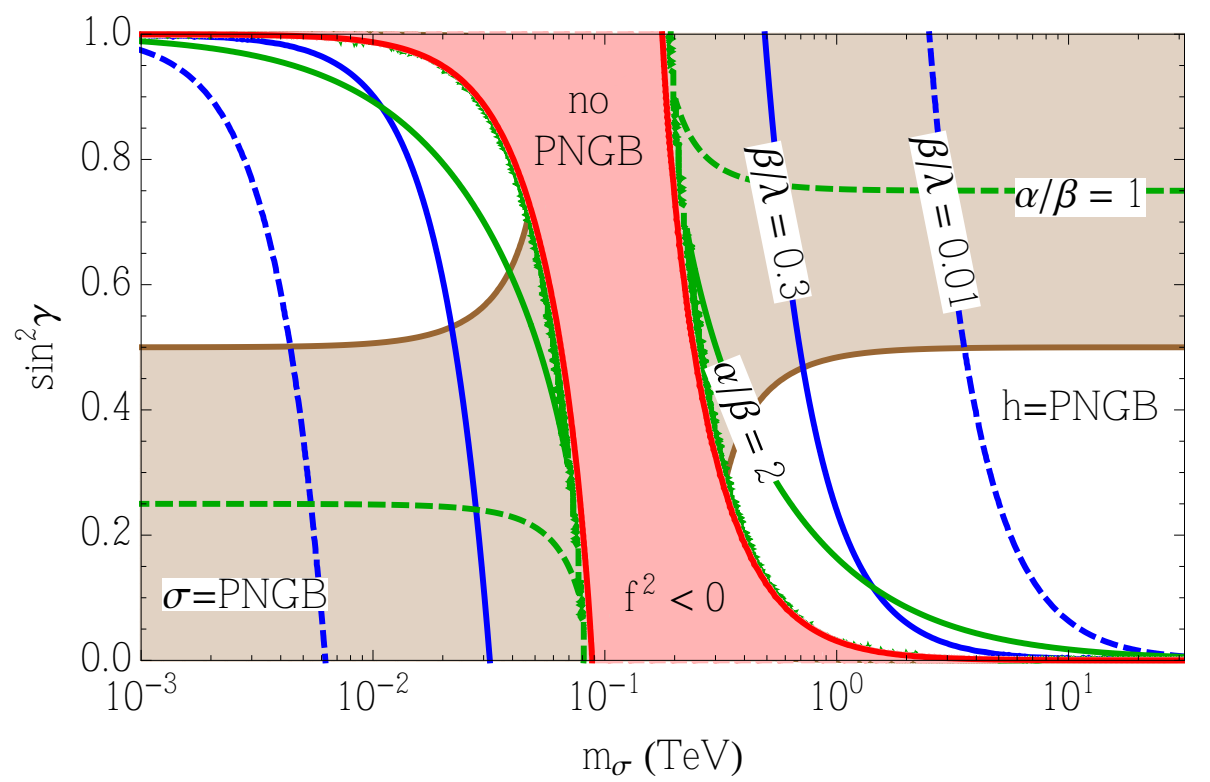

Figure 8.2: $m_{\sigma}$ versus $\sin ^{2} \gamma$ parameter space of the scalar sector. The Higgs mass $m_{h}$ and the Higgs vev $v$ have been fixed to their physical values. The red region corresponds to $f^{2}<0$, where spontaneous symmetry breaking is lost. In the brown region, $|v|>\left|v_{\sigma}\right|$. The Higgs is a $\mathrm{pGB}$ within the white regions at the bottom-right and the top-left part of the plane. The relative importance of the explicit breaking is shown through the $\beta / \lambda$ and $\alpha / \beta$ curves.

at $2 \sigma \mathrm{CL}$; which leaves as allowed parameter space a fraction of the lower white section of the figure. The unrotated Higgs field has the smaller vev in that region, so that for small mixings the physical Higgs is mostly the pGB (where the Higgs is mostly the pGB; $h \simeq \hat{h}$ for very small $\sin \gamma$ ), with a mass driven by $v$; while the physical $\sigma$ is mostly the scalar whose mass is due to the spontaneous breaking of $S O(5)$. The analysis in the next sections will thus focus in this regime, for which Fig. 8.2 already suggests a lower bound on $m_{\sigma}$ of around $550 \mathrm{GeV}$. Notice the blue contours indicating that the heavier the sigma, the softer the explicit breaking.

- Turning to $m_{h}>m_{\sigma}$ (left of the figure), the physical Higgs and sigma particles have interchanged their roles as the light and heavy particles:

$$
\begin{aligned}
h_{\text {phys }} & =\hat{\sigma} \cos \gamma+\hat{h} \sin \gamma, \\
\sigma_{\text {phys }} & =\hat{h} \cos \gamma-\hat{\sigma} \sin \gamma,
\end{aligned}
$$

The physical Higgs couplings to SM particles are now suppressed by $\sin \gamma$; which means that in this case the bound from LHC Higgs data is $\sin ^{2} \gamma>0.82$ at $2 \sigma \mathrm{CL}$. This leaves as allowed area only the upper part of the upper white region; where the smaller vev is that of the unrotated Higgs field. Interestingly, in the region where the mixing is $\sin \gamma$ is large, the physical Higgs is again mostly the particle getting the smaller vev, $h \simeq \hat{h}$, and thus it behaves mostly as the pGB. Nevertheless, the quartic coupling $\lambda$ is there very small, typically 
$\lambda<10^{-3}$, making the $S O(5)$ invariant potential very flat and thus potentially unstable against radiative corrections. Furthermore, if the soft breaking parameters are required to be small $\alpha, \beta \ll \lambda$, their values may require even more fine-tuning. For these reasons we will not study this case further; even if it seems an intriguing possibility.

\subsection{Fermionic sector}

Let us now describe the fermionic sector of the model. This is introduced in order to implement "partial compositeness" [35], as it is explained below and break in this way the $S O(5)$ symmetry explicitly.

Moreover, fermions are always a delicate sector; since they usually contribute to tensions with the EW scale (i.e. to the EW hierarchy problem) or make difficulties in BSM theories (flavor problem). Several possibilities for heavy BSM fermions embedded in $S O(5)$ have been explored in the literature for the exotic fermionic representations (see for instance Refs. [32,34]). For phenomenological reasons (for instance, the EW precision parameters) it is convenient to employ a model with heavy fermions with vectorial interactions under the SM gauge group, in such a way that the ordinary fermions couple to the Higgs at least through a heavy fermion so the light fermion masses will come suppressed by the heavy fermion scale; constituting in this way like a generalized seesaw pattern; as in Fig. 8.3. Therefore, the heavier the exotic fermions, the lighter the light fermions.

In particular, the setup considered has the following characteristics:

- There are heavy (exotic) vector-like fermions in complete representations of $S O(5)$, both in the fundamental representation, denoted below by $\psi$, or singlets denoted by $\chi$.

- By construction only the heavy exotic fermions couple directly to the scalar $\phi \|$, containing the $h$ and $\sigma$ particles.

- The Higgs field couples to the exotic fermions only via $S O(5)$ invariant Yukawa couplings. The sources of $S O(5)$ explicit breaking are instead the EW gauge interactions and the mixing terms between the heavy exotic fermions and the SM fermions. The explicit breaking produces an effective scalar potential at one-loop; which is modeled by the two soft breaking terms in Eq. (8.10); as is justified in Sect. 8.3.

The choice of fermionic representations respects an approximate custodial symmetry which protects the $Z b b$ coupling [111]. For simplicity, in the following we will deal only with the third generation SM quarks, top and bottom, which are expected to feel the effects of the "partial compositeness" the most. It would be straightforward to extend the results to the other quark flavors and leptons; for instance

\footnotetext{
"While in our model ordinary fermions never couple directly to the scalars; other models consider setups in which the SM left doublets are also embedded in $S O(5)$ multiplets together with heavy fermions. An example can be found in Ref. [34].
} 


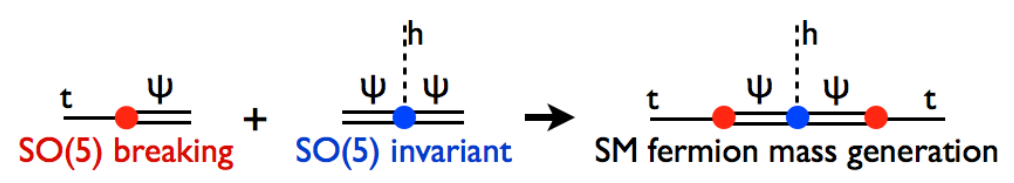

Figure 8.3: Schematics of light fermion mass generation. On the left part, the SM top quark depicted here as an example couples to the corresponding heavy partners breaking explicitly $S O(5)$. The middle picture shows the $S O(5)$ invariant Yukawa interactions between the Higgs and the heavy partners. The combination of both couplings induces an effective top Yukawa coupling and thus a massive top quark.

introducing heavier replica of the exotic sector, leading to very minor additional phenomenological impact.

In composite Higgs models there is a subtlety we have not introduced so far and will usually omit for simplicity: the $S O(5)$ is customarily enlarged by at least an extra $U(1)_{X}$ sector in order to ensure that the heavy exotic fields coupling directly with SM fermions have the right hypercharge assignments. This leads to a pattern of spontaneous global symmetry breaking given by:

$$
S O(5) \times U(1)_{X} \rightarrow S O(4) \times U(1)_{X} \approx S U(2)_{L} \times S U(2)_{R} \times U(1)_{X}
$$

with hypercharge corresponding to a combination of the charge under the new abelian generator $(X)$ and the third generator of $S U(2)_{R}\left(\mathrm{~T}_{R}^{3}\right)$ :

$$
Y=\mathrm{T}_{R}^{3}+X
$$

Since the global $U(1)_{X}$ symmetry remains unbroken, no additional Goldstone bosons are generated. Two different $U(1)_{X}$ charges are compatible with SM hypercharge assignments: $2 / 3$ and $-1 / 3$. We will thus consider two different copies of heavy fermions for each representation, each with a different charge under $U(1)_{X}$. It will turn out that each of them will be necessary to induce a mass term for the SM up-type quarks and down-type quarks, respectively.

Schematically, the fundamental and singlet representations can be decomposed under $S U(2)_{L}$ quantum numbers as follows:

$$
\begin{aligned}
\psi^{(2 / 3)} & \sim\left(X, Q, T^{(5)}\right), & \psi^{(-1 / 3)} & \sim\left(Q^{\prime}, X^{\prime}, B^{(5)}\right), \\
\chi^{(2 / 3)} & \sim T^{(1)}, & \chi^{(-1 / 3)} & \sim B^{(1)},
\end{aligned}
$$

where $X^{\left({ }^{\prime}\right)}, Q^{\left({ }^{\prime}\right)}$ denote the two different $S U(2)_{L}$ doublets contained in the fundamental representation of $S O(5)$. In each multiplet, the first doublet has $\mathrm{T}_{R}^{3}=1 / 2$ while the second one has $\mathrm{T}_{R}^{3}=-1 / 2$. Thus $Q$ and $Q^{\prime}$ are the heavy partners of the SM quark doublet; now denoted by lowercase, $q$, in order not to be confused. See Table 8.1 for a summary with the relevant quantum numbers for all heavy fermions.

On the other hand, $T^{(1,5)}, B^{(1,5)}$ denote $S U(2)_{L} \times S U(2)_{R}$ singlets, in the 5 and 1 representation of $S O(5)$, as it is indicated as superscript. $T_{R}^{(1,5)}$ have the same charges under the SM as $t_{R}$; while $B_{R}^{(1,5)}$ are the partners of $b_{R}$. 


\begin{tabular}{|c|c|c|c|c|c|c|}
\cline { 2 - 7 } \multicolumn{1}{c|}{} & $X$ & $Q$ & $T^{(1,5)}$ & $Q^{\prime}$ & $X^{\prime}$ & $B^{(1,5)}$ \\
\hline$\Sigma_{R}^{(3)}$ & $+1 / 2$ & $-1 / 2$ & 0 & $+1 / 2$ & $-1 / 2$ & 0 \\
\hline$S U(2)_{L} \times U(1)_{Y}$ & $(2,+7 / 6)$ & $(2,+1 / 6)$ & $(1,+2 / 3)$ & $(2,+1 / 6)$ & $(2,-5 / 6)$ & $(1,-1 / 3)$ \\
\hline$U(1)_{X}$ & $+2 / 3$ & $+2 / 3$ & $+2 / 3$ & $-1 / 3$ & $-1 / 3$ & $-1 / 3$ \\
\hline$U(1)_{E M}$ & $X^{u}=+5 / 3$ & $Q^{u}=+2 / 3$ & $+2 / 3$ & $\begin{array}{l}Q^{\prime u}=+2 / 3 \\
X^{\prime d}=-1 / 3\end{array}$ & $X^{\prime u}=-1 / 3$ & $-1 / 3$ \\
& $X^{d}=+2 / 3$ & $Q^{d}=-1 / 3$ & & $Q^{\prime d}=-4 / 3$ & \\
\hline
\end{tabular}

Table 8.1: Heavy fermion charges assignments under the different groups. $\Sigma_{R}^{(3)}$ is the third generator of $S U(2)_{R}$. The last row stands for the charges under the electromagnetic (EM) symmetry. Note there are two heavy fermions with exotic electric charges, $X^{u}$ and $X^{\prime d}$.

\section{The fermionic Lagrangian}

As stated above, the analysis below will be restricted to the third generation of SM quarks for simplicity, denoting by $q_{L}$ and $t_{R}$ and $b_{R}$ the doublet and singlets, respectively. Assuming the content specified in the previous section, the fermionic Lagrangian is given by

$$
\begin{aligned}
\mathcal{L}_{F}= & \bar{q}_{L} i \not D q_{L}+\bar{t}_{R} i \not D t_{R}+\bar{b}_{R} i \not D b_{R} \\
+ & \bar{\psi}^{(2 / 3)}\left(i \not D-M_{5}\right) \psi^{(2 / 3)}+\bar{\psi}^{(-1 / 3)}\left(i \not D-M_{5}^{\prime}\right) \psi^{(-1 / 3)} \\
+ & \bar{\chi}^{(2 / 3)}\left(i \not D-M_{1}\right) \chi^{(2 / 3)}+\bar{\chi}^{(-1 / 3)}\left(i \not D-M_{1}^{\prime}\right) \chi^{(-1 / 3)} \\
- & {\left[y_{1} \bar{\psi}_{L}^{(2 / 3)} \phi \chi_{R}^{(2 / 3)}+y_{2} \bar{\psi}_{R}^{(2 / 3)} \phi \chi_{L}^{(2 / 3)}\right.} \\
& +y_{1}^{\prime} \bar{\psi}_{L}^{(-1 / 3)} \phi \chi_{R}^{(-1 / 3)}+y_{2}^{\prime} \bar{\psi}_{R}^{(-1 / 3)} \phi \chi_{L}^{(-1 / 3)} \\
& +\Lambda_{1}\left(\bar{q}_{L} \Delta_{2 \times 5}^{(2 / 3)}\right) \psi_{R}^{(2 / 3)}+\Lambda_{2} \bar{\psi}_{L}^{(2 / 3)}\left(\Delta_{5 \times 1}^{(2 / 3)} t_{R}\right)+\Lambda_{3} \bar{\chi}_{L}^{(2 / 3)} t_{R} \\
& +\Lambda_{1}^{\prime}\left(\bar{q}_{L} \Delta_{2 \times 5}^{(-1 / 3)}\right) \psi_{R}^{(-1 / 3)}+\Lambda_{2}^{\prime} \bar{\psi}_{L}^{(-1 / 3)}\left(\Delta_{5 \times 1}^{(-1 / 3)} b_{R}\right)+\Lambda_{3}^{\prime} \bar{\chi}_{L}^{(-1 / 3)} b_{R} \\
& + \text { h.c. }] .
\end{aligned}
$$

The first line contains the kinetic terms for the SM fermions. The second and third lines include the kinetic and mass terms for the exotic fermions; which become $S O(5)$-invariant in the gauge-less limit. The fourth and fifth lines contain the $S O(5)$ invariant Yukawa couplings of the exotic sector to the Higgs field. Finally, the last three lines of the Lagrangian contain the $S O(5)$ explicit breaking interactions of SM fermions with exotic fermions. $\Delta_{2 \times 5}$ and $\Delta_{5 \times 1}$ are just introduced for formality and they denote suitable spurions to select the right fermions inside the $S O(5)$ multiplets, transforming under $S U(2) \times U(1)$ such that they mix with the corresponding SM fermion.

The primed parameters are connected to the bottom quark; therefore, if set to zero, no bottom mass would be generated through this mechanism. All parameters in Eq. (8.35) are assumed real for simplicity; i.e. we will assume CP invariance in what follows. 
It is useful to rewrite the Lagrangian in Eq.(8.35) in terms of $S U(2)_{L}$ components.

$$
\begin{aligned}
& \mathcal{L}_{F}= \bar{q}_{L} i \not D q_{L}+\bar{t}_{R} i \not D t_{R}+\bar{b}_{R} i \not D b_{R}+\bar{Q}\left(i \not D-M_{5}\right) Q+\bar{X}\left(i \not D-M_{5}\right) X \\
&+ \bar{T}^{(5)}\left(i \not D-M_{5}\right) T^{(5)}+\bar{T}^{(1)}\left(i \not D-M_{1}\right) T^{(1)}+\bar{Q}^{\prime}\left(i \not D-M_{5}^{\prime}\right) Q^{\prime} \\
&+ \bar{X}^{\prime}\left(i \not D-M_{5}^{\prime}\right) X^{\prime}+\bar{B}^{(5)}\left(i \not D-M_{5}^{\prime}\right) B^{(5)}+\bar{B}^{(1)}\left(i \not D-M_{1}^{\prime}\right) B^{(1)} \\
&-\left[y_{1}\left(\bar{X}_{L} H T_{R}^{(1)}+\bar{Q}_{L} \widetilde{H} T_{R}^{(1)}+\bar{T}_{L}^{(5)} \sigma T_{R}^{(1)}\right)\right. \\
& \quad+y_{2}\left(\bar{T}_{L}^{(1)} H^{\dagger} X_{R}+\bar{T}_{L}^{(1)} \widetilde{H}^{\dagger} Q_{R}+\bar{T}_{L}^{(1)} \sigma T_{R}^{(5)}\right) \\
& \quad+y_{1}^{\prime}\left(\bar{X}_{L}^{\prime}{ }_{L} \widetilde{H} B_{R}^{(1)}+\bar{Q}_{L}^{\prime} H B_{R}^{(1)}+\bar{B}_{L}^{(5)} \sigma B_{R}^{(1)}\right) \\
& \quad+y_{2}^{\prime}\left(\bar{B}_{L}^{(1)} \widetilde{H}^{\dagger} X_{R}^{\prime}+\bar{B}_{L}^{(1)} H^{\dagger} Q_{R}^{\prime}+\bar{B}_{L}^{(1)} \sigma B_{R}^{(5)}\right) \\
& \quad+\Lambda_{1} \bar{q}_{L} Q_{R}+\Lambda_{1}^{\prime} \bar{q}_{L} Q_{R}^{\prime}+\Lambda_{2} \bar{T}_{L}^{(5)} t_{R}+\Lambda_{3} \bar{T}_{L}^{(1)} t_{R} \\
&\left.\quad+\Lambda_{2}^{\prime} \bar{B}_{L}^{(5)} b_{R}+\Lambda_{3}^{\prime} \bar{B}_{L}^{(1)} b_{R}+h . c .\right] .
\end{aligned}
$$

Unless stated otherwise, here and in what follows, the $\sigma$ and $H(h)$ fields denote for simplicity the unshifted and unrotated original scalar fields of Eq. (8.10). This equation shows that the light fermion masses must be proportional to the $S O(5)$ invariant Yukawa couplings of heavy fermions and to the explicitly $S O(5)$ breaking light- heavy fermionic interactions; involving at least the vev for the scalar doublet $H$. Schematically, a $\bar{t}_{L} t_{R}$ mass term is seen to result from the following chain of couplings,

$$
q_{l} \underset{\Lambda_{1}}{\longrightarrow} Q_{R} \underset{M_{5}}{\longrightarrow} Q_{L} \underset{y_{1}\langle\tilde{H}\rangle}{\longrightarrow} T_{R}^{(1)} \underset{M_{1}}{\longrightarrow} T_{L}^{(1)} \underset{\Lambda_{3}}{\longrightarrow} t_{R}
$$

suggesting that the dominant contribution to the mass is

$$
m_{t} \propto y_{1} \frac{\Lambda_{1} \Lambda_{3}}{M_{1} M_{5}} v
$$

see also Fig. 8.3 and Sect. 10.2.1. Furthermore, both the $+2 / 3$ and $-1 / 3$ electrically charged sectors acquire off-diagonal mixing terms due to the linear fermion mixings and the Yukawa couplings.

The expression for the fermionic Lagrangian of Eq. (8.36) can be rewritten in a compact form defining a fermionic vector whose components are ordered by their electrical charges $q_{E M}=(+5 / 3,+2 / 3,-1 / 3,-4 / 3)$ :

$$
\Psi=\left(X^{u}, \mathcal{T}, \mathcal{B}, X^{\prime d}\right)
$$

where $\mathcal{T}$ and $\mathcal{B}$ include the top and bottom quarks together with their heavy fermionic partners:

$$
\mathcal{T}=\left(t, Q^{u}, X^{d}, T^{(5)}, T^{(1)}, Q^{\prime u}\right), \quad \mathcal{B}=\left(b, Q^{\prime d}, X^{\prime u}, B^{(5)}, B^{(1)}, Q^{d}\right) .
$$

The fermion mass terms can then be written as

$$
\mathcal{L}_{\mathcal{M}}=-\bar{\Psi}_{L} \mathcal{M}(h, \sigma) \Psi_{R}
$$


where here and in what follows the sum over all components of the fermionic vector is left implicit and the block diagonal $14 \times 14$ fermion mass matrix $\mathcal{M}$ reads

$$
\begin{aligned}
\mathcal{M}(h, \sigma) & =\operatorname{diag}\left(M_{5}, \mathcal{M}^{\mathcal{T}}(h, \sigma), \mathcal{M}^{\mathcal{B}}(h, \sigma), M_{5}^{\prime}\right) \\
\mathcal{M}^{\mathcal{T}}(h, \sigma) & =\left(\begin{array}{cccccc}
0 & \Lambda_{1} & 0 & 0 & 0 & \Lambda_{1}^{\prime} \\
0 & M_{5} & 0 & 0 & y_{1} \frac{h}{\sqrt{2}} & 0 \\
0 & 0 & M_{5} & 0 & y_{1} \frac{h}{\sqrt{2}} & 0 \\
\Lambda_{2} & 0 & 0 & M_{5} & y_{1} \sigma & 0 \\
\Lambda_{3} & y_{2} \frac{h}{\sqrt{2}} & y_{2} \frac{h}{\sqrt{2}} & y_{2} \sigma & M_{1} & 0 \\
0 & 0 & 0 & 0 & 0 & M_{5}^{\prime}
\end{array}\right), \\
\mathcal{M}^{\mathcal{B}}(h, \sigma) & =\mathcal{M}^{\mathcal{T}}(h, \sigma) \text { with }\left\{y_{i}, \Lambda_{i}, M_{i}\right\} \leftrightarrow\left\{y_{i}^{\prime}, \Lambda_{i}^{\prime}, M_{i}^{\prime}\right\} .
\end{aligned}
$$

The mass matrices can be diagonalized by bi-unitary (or bi-orthogonal, since the parameters are real) transformations:

$$
\Psi_{L}^{\text {phys }}=L \Psi_{L}, \quad \Psi_{R}^{\text {phys }}=R \Psi_{R}, \quad \mathcal{M}^{\text {diag }}=L^{\dagger} \mathcal{M} R
$$

$\mathcal{M}^{\mathcal{T}}$ and $\mathcal{M}^{\mathcal{B}}$ are rather complicated matrices. Therefore, we can diagonalize them only in very simple limits; in which some of the parameters are set to zero; but in order to be general, in the phenomenological analysis of the next chapter they will be diagonalized numerically.

Notice that after the diagonalization, the physical light eigenstates will be admixtures of the light and heavy fermion fields appearing in the Lagrangian and that the vevs of the scalar fields will also induce heavy fermion mass splittings.

\subsection{Coleman-Weinberg potential}

In Sect. 8.1 it was assumed a specific form for the $S O(5)$ scalar potential broken to $S O(4)$, introducing two additional $S O(5)$ breaking parameters $\alpha$ and $\beta$. In this section we will further motivate this assumption. Even assuming that the tree level scalar potential would preserve the global $S O(5)$ symmetry, the presence of $S O(5)$ breaking couplings in the fermionic sector will generate at one-loop level $S O(5)$ breaking terms through the Coleman-Weinberg mechanism [187].

The sources of $S O(5)$ explicit breaking in the model are the $S U(2)_{L} \times U(1)_{Y}$ gauge couplings in the covariant derivative and the linear mixings between heavy and light fermions described in the previous section. The one-loop fermionic contribution is computed as all possible 1PI one-loop diagrams for $2 n$ scalar legs (figure taken from [187]):

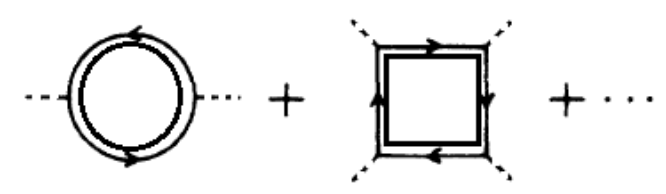


Therefore it can be obtained from the field dependent mass matrix $\mathcal{M}$ as

$$
\begin{aligned}
V_{\text {loop }}=-\frac{i}{2} \int \frac{d^{4} k}{(2 \pi)^{4}} \sum_{n=1}^{\infty} \frac{1}{n} \operatorname{Tr}\left(\frac{\mathcal{M} \mathcal{M}^{\dagger}}{k^{2}}\right)^{n}=\frac{i}{2} \int \frac{d^{4} k}{(2 \pi)^{4}} \operatorname{Tr} \log \left(1-\frac{\mathcal{M M}^{\dagger}}{k^{2}}\right) \\
=-\frac{1}{64 \pi^{2}}\left(\Lambda^{2} \operatorname{Tr}\left[\mathcal{M} \mathcal{M}^{\dagger}\right]-\operatorname{Tr}\left[\left(\mathcal{M} \mathcal{M}^{\dagger}\right)^{2}\right] \log \left(\frac{\Lambda^{2}}{\mu^{2}}\right)+\right. \\
\left.+\operatorname{Tr}\left[\left(\mathcal{M M}^{\dagger}\right)^{2} \log \left(\frac{\mathcal{M M}^{\dagger}}{\mu^{2}}\right)\right]-\frac{1}{2} \operatorname{Tr}\left[\left(\mathcal{M} \mathcal{M}^{\dagger}\right)^{2}\right]\right),
\end{aligned}
$$

where $\Lambda$ is the UV cutoff scale while $\mu$ is a generic renormalization scale. As a result, one quadratically UV divergent term $\left(\Lambda^{2}\right)$ and one logarithmically UV divergent term $(\log \Lambda)$ appear; being the rest of contributions finite. For the model under discussion the form of the UV divergent terms in terms of the scalars is:

$$
\begin{aligned}
\operatorname{Tr}\left[\mathcal{M M}^{\dagger}\right] & =c_{1}+c_{2}\left(\phi^{T} \phi\right), \\
\operatorname{Tr}\left[\left(\mathcal{M} \mathcal{M}^{\dagger}\right)^{2}\right] & =d_{1}+d_{2} \sigma+d_{3} h^{2}+d_{4}\left(\phi^{T} \phi\right)+d_{5}\left(\phi^{T} \phi\right)^{2},
\end{aligned}
$$

where the explicit expressions for the coefficients are

$$
\begin{aligned}
& c_{1}=2 \Lambda_{1}^{2}+\Lambda_{2}^{2}+\Lambda_{3}^{2}+M_{1}^{2}+5 M_{5}^{2}+\left(\{\} \longrightarrow\{\}^{\prime}\right) \\
& c_{2}=y_{1}^{2}+y_{2}^{2}+\left(\{\} \longrightarrow\{\}^{\prime}\right),
\end{aligned}
$$

and

$$
\begin{aligned}
& d_{1}=M_{1}^{4}+5 M_{5}^{4}+2 M_{5}^{2}\left(2 \Lambda_{1}^{2}+\Lambda_{2}^{2}\right)+2 M_{1}^{2} \Lambda_{3}^{2}+2 \Lambda_{1}^{4}+\left(\Lambda_{2}^{2}+\Lambda_{3}^{2}\right)^{2}+\left(\{\} \longrightarrow\{\}^{\prime}\right), \\
& d_{2}=4\left(y_{1} M_{1}+y_{2} M_{5}\right) \Lambda_{2} \Lambda_{3}+\left(\{\} \longrightarrow\{\}^{\prime}\right), \\
& d_{3}=-2 y_{1}^{2} \Lambda_{2}^{2}+y_{2}^{2} \Lambda_{1}^{2}+\left(\{\} \longrightarrow\{\}^{\prime}\right), \\
& d_{4}=4 y_{1} y_{2} M_{1} M_{5}+2\left(y_{1}^{2}+y_{2}^{2}\right)\left(M_{1}^{2}+M_{5}^{2}\right)+2 y_{1}^{2} \Lambda_{2}^{2}+2 y_{2}^{2} \Lambda_{3}^{2}+\left(\{\} \longrightarrow\{\}^{\prime}\right), \\
& d_{5}=y_{1}^{4}+y_{2}^{4}+\left(\{\} \longrightarrow\{\}^{\prime}\right),
\end{aligned}
$$

where the $\left(\{\} \longrightarrow\{\}^{\prime}\right)$ notation means there is a term with all parameters being primed for each of the non-primed contributions shown.

From Eqs. (8.47) and (8.48), the quadratically divergent piece is seen to be $S O(5)$ invariant; while the logarithmic divergence contains two terms $\left(\sigma\right.$ and $\left.h^{2}\right)$ breaking $S O(5)$ explicitly; while respecting $S O(4)$ (in an arbitrary gauge, remember $h^{2}$ would correspond to the combination $\left.h^{2}+\pi^{2}\right)$.

We can get rid of the $S O(5)$ invariant contributions via redefinitions of the parameters of the tree-level Lagrangian, and thus the quadratic divergence can be fully absorbed and the same holds for the $S O(5)$ invariant components of the logarithmically divergent terms $\left(d_{1}, d_{4}\right.$ and $\left.d_{5}\right)$. However, the presence of the $d_{2}$ and $d_{3}$ divergent $S O(5)$-breaking terms requires to add two corresponding counterterms in the potential, so the theory can be renormalized. These two necessary terms are those defined with coefficients $\alpha$ and $\beta$ in the potential; see Eq. (8.10). The computation of the finite part of $V_{\text {loop }}$ should provide the dependence of the parameters on the renormalization scale. 
On the other hand, the SM gauge couplings, despite breaking explicitly the $S O(5)$ symmetry, do not induce extra one-loop divergent contributions to the effective potential other than those already present in the SM. Furthermore, the finite contributions from the EW gauge bosons; going as their mass scale, can be neglected with respect to the ones from heavy fermions, which go as the heavy fermion mass scale. Therefore, being irrelevant for the scope of our work, we will only speak of contributions to the scalar one-loop potential from heavy fermions. 


\section{Chapter 9}

\section{Phenomenology}

The phenomenology of the model presented in the previous chapter is explored here. Bounds are derived first on the model parameters resulting from EW precision tests (namely $\mathrm{S}, \mathrm{T}$ and $g_{L}^{b}$ ) and from LHC Higgs data.

We will analyze the possibility of having a $\sigma$ particle in LHC data and, in that case, what could distinguish the phenomenology from an $S O(5) / S O(4)$ model from that of a generic singlet scalar. For this we will focus on a sigma particle with mass above the EW scale, but below $1 \mathrm{TeV}$, lighter than the heavy exotic fermions; while the analysis of the implications in the limit of very heavy sigma mass will be undertaken in the next chapter.

\subsection{Bound from Higgs measurements}

Current data from Higgs measurements at LHC already allow us to constrain the mixing between the $\sigma$ and the Higgs $h$ particles. In particular, the bound comes from $h$ decays to the EW gauge bosons and the effective $h$-gluon-gluon coupling. Both couplings are suppressed at leading order by a $\cos \gamma$ factor with respect to the SM value (see Sects. 8.1.2 and 9.3). We use the latest ATLAS and CMS combined results for the gluon-gluon and vector boson mediated Higgs production processes [67].

A $\chi^{2}$ fit taking into account the correlation between the corresponding coupling modifiers in the combined fit of the 7 and $8 \mathrm{TeV}$ LHC data (given by figure 26.A of Ref. [67]) constrains directly $\cos \gamma$, translating into the following bound

$$
\sin ^{2} \gamma \lesssim 0.18(\text { at } 2 \sigma)
$$

which in the $m_{\sigma} \gg m_{h}$ limit would point to a value for the non-linearity parameter of composite Higgs models, $\xi \equiv v^{2} / f^{2} \sim \sin ^{2} \gamma$, see Eqs. (8.30) and (8.31). This is consistent with the limits found in the literature $[32,33,101]^{*}$.

\footnotetext{
*The fermion configuration in our model differs from that of the standard $M C H M_{5}$ composite
} 


\subsection{Precision electroweak constraints}

Analyses available on precision tests for composite Higgs models, such as that in Ref. [188], usually consider non-linear versions of the theory where the only scalar present is the Higgs particle, but for Ref. [34], which discusses qualitatively the interplay of scalar and exotic fermion contributions. See also Ref. [189] in the context of a $S U(4) / S p(4)$ model. We present here an analysis of the impact from the scalars $(h$ and $\sigma$ ) and the heavy fermions on precision parameters, namely $S, T$ and the $Z \bar{b}_{L} b_{L}$ coupling. This will allow us to discuss the impact of varying the $\sigma$ mass and some other parameter correlations.

\section{$S, T$ and $g_{L}^{b}$}

Consider the parameter definitions in Ref. [177]:

$$
\begin{aligned}
& \alpha S=\left.4 s_{W} c_{W} \frac{d \Pi_{30}\left(q^{2}\right)}{d q^{2}}\right|_{q^{2}=0}=4 s_{W} c_{W} F_{30}, \\
& \alpha T=\frac{1}{M_{W}^{2}}\left[\Pi_{11}(0)-\Pi_{33}(0)\right]=\frac{1}{M_{W}^{2}}\left[A_{11}-A_{33}\right], \\
& \alpha U=-\left.4 s_{W}^{2} \frac{d}{d q^{2}}\left[\Pi_{33}\left(q^{2}\right)-\Pi_{11}\left(q^{2}\right)\right]\right|_{q^{2}=0}=4 s_{W}^{2}\left(F_{11}-F_{33}\right),
\end{aligned}
$$

where $c_{W}\left(s_{W}\right)$ denotes the cosinus (sinus) of the Weinberg angle $c_{W}=M_{W} / M_{Z}$, and the electroweak vacuum polarization functions are given by

$$
\Pi_{i j}^{\mu \nu}(q)=-i\left[\Pi_{i j}\left(q^{2}\right) g^{\mu \nu}+\left(q^{\mu} q^{\nu}-\text { terms }\right)\right] ; \quad \Pi_{i j}\left(q^{2}\right) \equiv A_{i j}(0)+q^{2} F_{i j}+\ldots
$$

with $i, j=W, Z$ or $i, j=0,3$ for the $B$ or the $W_{3}$ bosons, respectively, and the dots indicating an expansion in powers of $q^{2}$. We will not consider further $U$ as it typically corresponds to higher order (mass dimension eight) couplings while only low-energy data (e.g. LEP) will be used here ${ }^{\dagger}$.

Furthermore, relevant constraints could also stem from deviations induced in the $Z \bar{b}_{L} b_{L}$ coupling from the heavy BSM fermion introduced. This is parametrized by $g_{L}^{b}$ in the decay amplitude:

$$
\mathcal{M}_{Z \rightarrow \bar{b}_{L} b_{L}}=-\frac{e g_{L}^{b}}{s_{W} c_{W}} \bar{b}\left(p_{2}\right) \notin(q) \frac{1-\gamma_{5}}{2} b\left(p_{1}\right)
$$

where $\epsilon(q)$ denotes the $Z$ boson polarization and $p_{i}$ the $b$ quark and antiquark momenta.

The values of $S, T$ and $g_{L}^{b}$ are allowed to deviate from the SM prediction within the constraints: $[188,191]$

$$
\begin{aligned}
\Delta S & \equiv S-S_{S M}=0.0079 \pm 0.095 \\
\Delta T & \equiv T-T_{S M}=0.084 \pm 0.062 \\
\Delta g_{L}^{b} & \equiv g_{L}^{b}-g_{L, S M}^{b}=(-0.13 \pm 0.61) \times 10^{-3},
\end{aligned}
$$

model, which results in a different dependence on $\xi$ of the coupling modifier to fermions $\kappa_{f}$; see Eq. (10.38) and Sect. 10.1 for more details.

$\dagger$ The correspondence with parameters in Ref. [190] is: $\epsilon_{1} \equiv \alpha T \epsilon_{3}=\alpha S /\left(4 s_{W}^{2}\right)$ 
with the $\left(S, T, g_{L}^{b}\right)$ correlation matrix given by

$$
\left(\begin{array}{ccc}
1 & 0.864 & 0.06 \\
0.864 & 1 & 0.123 \\
0.06 & 0.123 & 1
\end{array}\right)
$$

\section{Scalar contributions in the linear $S O(5)$ model: $h$ and $\sigma$}

Given the scalar couplings in Eq. (8.21), their contributions to $S$ and $T$ can be formulated as

$$
\begin{array}{r}
\Delta T^{(h \text { and } \sigma)}=-\Delta T_{S M}^{h}\left(m_{h}\right)+c_{\gamma}^{2} \Delta T_{S M}^{h}\left(m_{h}\right)+\Delta T^{(\sigma)} \\
=s_{\gamma}^{2}\left[-\Delta T_{S M}^{h}\left(m_{h}\right)+\Delta T_{S M}^{h}\left(m_{\sigma}\right)\right], \\
\begin{array}{r}
\Delta S^{(h \text { and } \sigma)}=-\Delta S_{S M}^{h}\left(m_{h}\right)+c_{\gamma}^{2} \Delta S_{S M}^{h}\left(m_{h}\right)+\Delta S^{(\sigma)} \\
=s_{\gamma}^{2}\left[-\Delta S_{S M}^{h}\left(m_{h}\right)+\Delta S_{S M}^{h}\left(m_{\sigma}\right)\right],
\end{array}
\end{array}
$$

where the $\sigma$ contributions $\Delta T^{(\sigma)}$ and $\Delta S^{(\sigma)}$ have been simply written in terms of the usual SM formulae for the Higgs contribution $\Delta T_{S M}^{h}$ and $\Delta S_{S M}^{h}$ with the replacement $m_{h} \rightarrow m_{\sigma}$. The scalar contribution to $\Delta T$ is then given by

$$
\begin{aligned}
\Delta T^{(h \text { and } \sigma)}= & \frac{3 G_{F} M_{W}^{2}}{8 \pi^{2} \sqrt{2}} s_{\gamma}^{2}\left(-m_{h}^{2} \frac{\log \left(m_{h}^{2} / M_{W}^{2}\right)}{M_{W}^{2}-m_{h}^{2}}+m_{\sigma}^{2} \frac{\log \left(m_{\sigma}^{2} / M_{W}^{2}\right)}{M_{W}^{2}-m_{\sigma}^{2}}\right. \\
& \left.+\frac{M_{Z}^{2}}{M_{W}^{2}}\left\{m_{h}^{2} \frac{\log \left(m_{h}^{2} / M_{Z}^{2}\right)}{M_{Z}^{2}-m_{h}^{2}}-m_{\sigma}^{2} \frac{\log \left(m_{\sigma}^{2} / M_{Z}^{2}\right)}{M_{Z}^{2}-m_{\sigma}^{2}}\right\}\right),
\end{aligned}
$$

which in the limit $m_{\sigma} \gg m_{h}, M_{W}, M_{Z}$ reduces to

$$
\Delta T^{(h \text { and } \sigma)} \sim s_{\gamma}^{2} \frac{3 G_{F} M_{W}^{2}}{8 \pi^{2} \sqrt{2}} \frac{s_{W}^{2}}{c_{W}^{2}} \log \left(m_{\sigma}^{2} / M_{W}^{2}\right) .
$$

For the $\Delta S$ corrections, the formulation in Refs. $[192,193]$ is used, leading to

$$
\begin{aligned}
\alpha \Delta S_{S M}^{h}(m)=s_{W}^{2} \frac{2 G_{F}}{\sqrt{2} \pi^{2}} M_{W}^{2}\left(\frac{x}{12(x-1)}\right. & \log (x)+\left(-\frac{x}{6}+\frac{x^{2}}{12}\right) F(x) \\
& \left.-\left(1-\frac{x}{3}+\frac{x^{2}}{12}\right) F^{\prime}(x)\right)
\end{aligned}
$$

where $x \equiv m^{2} / M_{Z}^{2}$ and for $x<4$ :

$$
\begin{aligned}
& F(x)=1+\left(\frac{x}{x-1}-\frac{1}{2} x\right) \log x-x \sqrt{\frac{4}{x}-1} \arctan \sqrt{\frac{4}{x}-1}, \\
& F^{\prime}(x)=-1+\frac{x-1}{2} \log x+(3-x) \sqrt{\frac{x}{4-x}} \arctan \sqrt{\frac{4}{x}-1},
\end{aligned}
$$




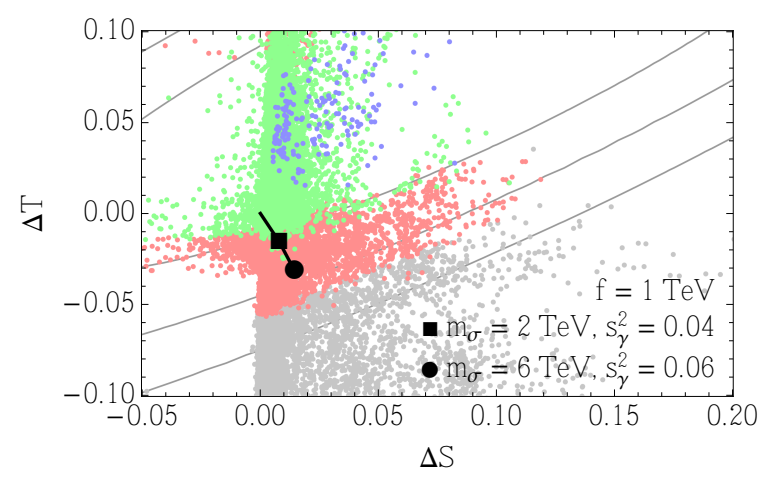

Figure 9.1: Uncombined contributions of the scalar sector (black curve) and the exotic fermionic sector to the parameters $S$ and $T$.

while for $x>4$ :

$$
\begin{aligned}
& F(x)=1+\left(\frac{x}{x-1}-\frac{1}{2} x\right) \log x-x \sqrt{1-\frac{4}{x}} \log \left(\sqrt{\frac{x}{4}-1}+\sqrt{\frac{x}{4}}\right), \\
& F^{\prime}(x)=-1+\frac{x-1}{2} \log x+(3-x) \sqrt{\frac{x}{x-4}} \log \left(\sqrt{\frac{x}{4}-1}+\sqrt{\frac{x}{4}}\right) .
\end{aligned}
$$

In the limit of very large $m_{\sigma}$, the $\sigma$ contribution to $S$ can be approximated by:

$$
\alpha \Delta S^{(\sigma)} \underset{\sigma \rightarrow \infty}{\longrightarrow} s_{\gamma}^{2} s_{W}^{2} \frac{2 G_{F}}{\sqrt{2} \pi^{2}} M_{W}^{2}\left[\frac{1}{12} \log \left(\frac{m_{\sigma}^{2}}{M_{W}^{2}}\right)\right],
$$

consistent with the statements in the literature for a very heavy Higgs particle [177].

In Fig. 9.1 the black points are shown to compare the impact from the $\sigma$ scalar on $S$ and $T$ when varying its mass. As explained in Sect. 8.1.3, the set of parameters in the scalar potential $(f, \lambda, \alpha, \beta)$ has been traded by four observables: $G_{F}, m_{h}, m_{\sigma}$ and the scalar mixing $\gamma$ (with the latter two yet unknown). The first point $\left(m_{\sigma}=2\right.$ $\left.\mathrm{TeV}, s_{\gamma}^{2}=0.04\right)$ corresponds to $\{f=1 \mathrm{TeV}, \lambda=0.38, \alpha=0.35, \beta=0.16\}$; i.e. it lies within the perturbative regime of the linear $S O(5)$ sigma model. On the other hand, the second point $\left(m_{\sigma}=6 \mathrm{TeV}, s_{\gamma}^{2}=0.06\right)$ implies $\{f=1 \mathrm{TeV}, \lambda=4.3$, $\alpha=0.25, \beta=0.13\}$. Now the scalar self-coupling $\lambda$ is closer to the limit of validity of the perturbative expansion $\left(m_{\sigma}=4 \pi f\right)$ [100]; and the $S O(5)$ explicit breaking is much softer in comparison. Both correspond to $f=1 \mathrm{TeV}$ and are within the soft explicit breaking regime $\alpha, \beta<\lambda$. Note that, for a $\sigma$ particle much heavier than the Higgs, values of $f$ below $700 \mathrm{GeV}$ would be difficult to accommodate experimentally as $\sin ^{2} \gamma \simeq v^{2} / f^{2}$, see Eq. (9.1).

In summary, Fig. 9.1 shows a sizable negative contribution of the $\sigma$ particle to $\Delta T$ which increases with $m_{\sigma}$, and positive contribution to $\Delta S$, also increasing with the mass. The result is consistent with the pattern expected in the qualitative discussion of Ref. [34], and similar to that for the heavy Higgs case (see e.g. Ref. [194]). In the limit $m_{\sigma} \rightarrow m_{h}$ the total scalar contribution matches that in the SM due to the Higgs particle. It is easy to extrapolate the $S$ and $T$ scalar contributions to other mixing regimes as they scale with $s_{\gamma}^{2}$. For instance, the effect would be amplified 


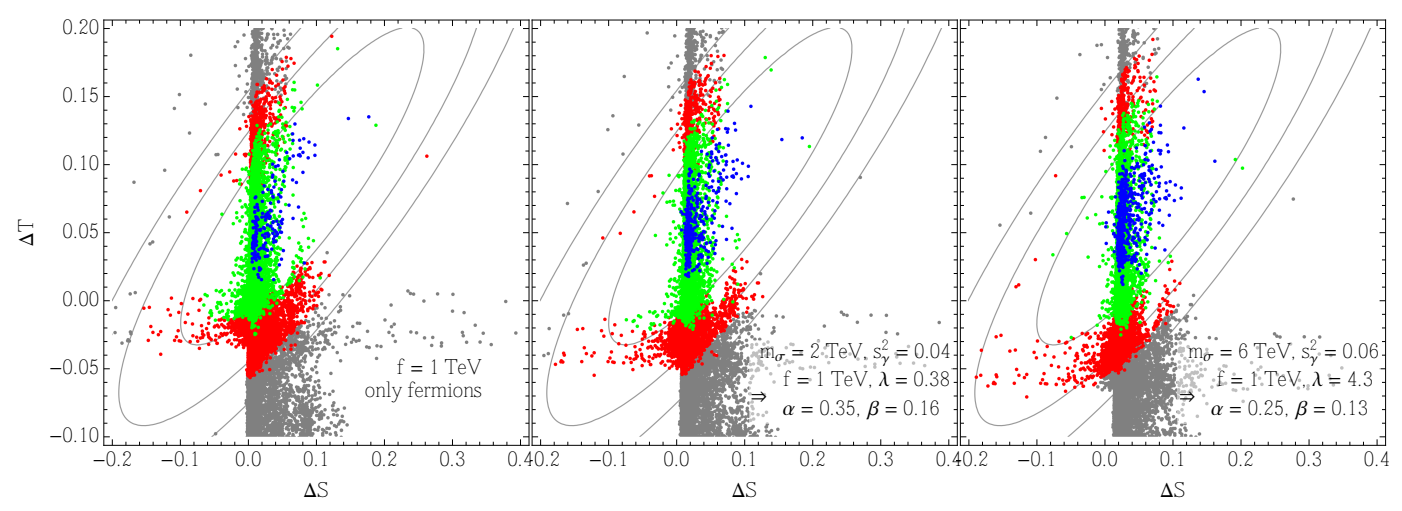

Figure 9.2: Combined contributions to $S$ and $T$ from the scalar sector and the exotic fermionic sector. The blue, green and red points are allowed at 1,2, $3 \sigma$ by the combined $\left(S, T, g_{L}^{b}\right)$ fit, while gray points are outside the $3 \sigma$ region.

by a factor of $\sim 3$ when raising the mixing towards the maximal value allowed by Higgs data, see Eq. (9.1).

Finally, for $g_{L}^{b}$ we will not analyze the one-loop $\sigma$ contributions, since they would be proportional to the bottom Yukawa couplings and thus negligible compared to the top and top-partner contributions to be discussed below.

\section{Fermionic contributions}

The heavy fermion sector may also have an impact on the $S$ and $T$ parameters and on $g_{L}^{b}$. This sector adds additional parameter dependence on top of the four renormalization parameters already discussed for the scalar sector of the linear $S O(5)$ sigma model. The fermionic parameter space is quite large and adjustable, and thus in practice $m_{\sigma}$ and $\gamma$ will be treated here as independent from them. It will also be assumed that the inclusion of quarks and leptons from the first two generations does not alter significantly the analysis of electroweak precision tests, as lighter fermions would have a much smaller mixing with their heavy partners.

The gauge boson couplings to neutral (NC) and charged (CC) fermionic currents in the weak basis can be read from the charges in Table 8.1. After rotation to the mass basis (see Sect. 8.2.1), the corresponding Lagrangians can be written as (the notation is similar to Ref. [195]):

$$
\begin{aligned}
\mathcal{L}_{\mathrm{NC}} & =\bar{\Psi}^{\mathrm{phys}} \gamma^{\mu}\left[\frac{g}{2}\left(C_{L} P_{L}+C_{R} P_{R}\right) W_{\mu}^{3}-g^{\prime}\left(Y_{L} P_{L}+Y_{R} P_{R}\right) B_{\mu}\right] \Psi^{\mathrm{phys}} \\
& =\bar{\Psi}^{\mathrm{phys}} \gamma^{\mu}\left[\frac{g}{2 c_{W}}\left(C_{L} P_{L}+C_{R} P_{R}-2 s_{W}^{2} \mathcal{Q}\right) Z_{\mu}-e \mathcal{Q} A_{\mu}\right] \Psi^{\mathrm{phys}} \\
\mathcal{L}_{C C} & =\bar{\Psi}^{\mathrm{phys}} \gamma^{\mu}\left[\frac{g}{\sqrt{2}}\left(V_{L} P_{L}+V_{R} P_{R}\right) W_{\mu}^{+}\right] \Psi^{\mathrm{phys}}+\text { h.c. }
\end{aligned}
$$

where $P_{L}$ and $P_{R}$ are chirality projectors, $\Psi^{\text {phys }}$ denotes the generic fermionic vector in the physical mass basis and $e$ is the absolute value of the electric charge unit. In 
the model under discussion, the matrices $C$ and $Y$ are related via the electric charge matrix -see also Eq. (8.33):

$$
Y_{\alpha}=\mathcal{Q}-\frac{1}{2} C_{\alpha} \quad \alpha=L \text { or } R
$$

with

$$
\mathcal{Q}=\left(+\frac{5}{3},+\frac{2}{3} \mathbb{1}_{6 \times 6},-\frac{1}{3} \mathbb{1}_{6 \times 6},-\frac{4}{3}\right)
$$

The relation between the $\mathrm{NC}$ coupling matrices in the mass basis, $C_{L, R}$ and $Y_{L, R}$, and their counterparts in the interaction basis (same symbols in curly characters below) is given by

$$
\begin{aligned}
& C_{L}=L \mathcal{C}_{L} L^{\dagger}, \quad C_{R}=R \mathcal{C}_{R} R^{\dagger}, \\
& \mathcal{C}_{L ; R}=\operatorname{diag}\left(+1, \mathcal{C}_{L ; R}^{\mathcal{T}}, \mathcal{C}_{L ; R}^{\mathcal{B}},-1\right), \\
& \mathcal{C}_{L ; R}^{\mathcal{T}}=-\mathcal{C}_{L ; R}^{\mathcal{B}}=\operatorname{diag}(+1 ; 0,+1,-1,0,0,+1) ; \\
& Y_{L}=L \mathcal{Y}_{L} L^{\dagger}, \quad Y_{R}=R \mathcal{Y}_{R} R^{\dagger}, \\
& \mathcal{Y}_{L ; R}=\operatorname{diag}\left(+\frac{7}{6}, \mathcal{Y}_{L ; R}^{\mathcal{T}}, \mathcal{Y}_{L ; R}^{\mathcal{B}},-\frac{5}{6}\right), \\
& \mathcal{Y}_{L ; R}^{\mathcal{T}}=\operatorname{diag}\left(\frac{1}{6} ; \frac{2}{3}, \frac{1}{6}, \frac{7}{6}, \frac{2}{3}, \frac{2}{3}, \frac{1}{6}\right), \\
& \mathcal{Y}_{L ; R}^{\mathcal{B}}=\operatorname{diag}\left(\frac{1}{6} ;-\frac{1}{3}, \frac{1}{6},-\frac{5}{6},-\frac{1}{3},-\frac{1}{3}, \frac{1}{6}\right) .
\end{aligned}
$$

Analogously, for the CC coupling matrices $V_{L, R}$ :

$$
\begin{aligned}
V_{L} & =L \mathcal{V} L^{\dagger}, \\
\mathcal{V}_{L ; R} & =\left(\begin{array}{cccc}
0 & V_{R}=R \mathcal{V} R^{\dagger} & \\
\mathbf{0}_{6 \times 1} & \mathbf{0}_{6 \times 6} & \mathbf{0}_{1 \times 6} \mathcal{T} \mathcal{B} & 0 \\
\mathbf{0}_{6 \times 1} & \mathbf{0}_{6 \times 6} & \mathbf{0}_{6 \times 6} & \mathcal{V}^{\mathcal{B} X^{\prime d}} \\
0 & \mathbf{0}_{1 \times 6} & \mathbf{0}_{1 \times 6} & 0
\end{array}\right), \\
\mathcal{V}^{X^{u} \mathcal{T}} & =\left(\mathcal{V}^{\mathcal{B} X^{\prime d}}\right)^{\dagger}=(0,0,1,0,0,0)
\end{aligned}
$$

while $\mathcal{V}_{L}^{\mathcal{T B}}$ is a $6 \times 6$ matrix whose elements are null but for its $(1,1),(2,6)$ and $(6,2)$ entries with value 1 , and $\mathcal{V}_{R}^{\mathcal{T} \mathcal{B}}$ is a $6 \times 6$ matrix with null elements but for its $(2,6)$ and $(6,2)$ entries with value 1.

The contribution of the fermionic sector to the $T$ parameter, $\Delta T^{f}$, is given by [196]:

$$
\begin{aligned}
\Delta T^{f}=\frac{3}{16 \pi s_{W}^{2} c_{W}^{2}} & \left\{\sum_{i j}\left[\left(\left(V_{L}^{i j}\right)^{2}+\left(V_{R}^{i j}\right)^{2}\right) \theta_{+}\left(\eta_{i}, \eta_{j}\right)+2 V_{L}^{i j} V_{R}^{i j} \theta_{-}\left(\eta_{i}, \eta_{j}\right)\right]-\right. \\
& \left.-\frac{1}{2} \sum_{i j}\left[\left(\left(C_{L}^{i j}\right)^{2}+\left(C_{R}^{i j}\right)^{2}\right) \theta_{+}\left(\eta_{i}, \eta_{j}\right)+2 C_{L}^{i j} C_{R}^{i j} \theta_{-}\left(\eta_{i}, \eta_{j}\right)\right]\right\} \\
& -\frac{3}{16 \pi s_{W}^{2} c_{W}^{2} M_{Z}^{2}}\left(m_{t}^{2}+m_{b}^{2}-2 \frac{m_{t}^{2} m_{b}^{2}}{m_{t}^{2}-m_{b}^{2}} \ln \frac{m_{t}^{2}}{m_{b}^{2}}\right)
\end{aligned}
$$


where $m_{i}$ denotes the fermion masses, $m_{i} \equiv \mathcal{M}_{i i}^{\text {diag }}$, and $\eta_{i} \equiv m_{i}^{2} / M_{Z}^{2}$. The last line in this equation corresponds to the subtraction of the SM contribution from the light fermions (top and bottom). The $\theta_{ \pm}$functions are defined as [196]:

$$
\begin{aligned}
\theta_{+}\left(\eta_{1}, \eta_{2}\right)= & \eta_{1}+\eta_{2}-\frac{2 \eta_{1} \eta_{2}}{\eta_{1}-\eta_{2}} \ln \frac{\eta_{1}}{\eta_{2}}-2\left(\eta_{1} \ln \eta_{1}+\eta_{2} \ln \eta_{2}\right) \\
& +\operatorname{div} \frac{\eta_{1}+\eta_{2}}{2} \\
\theta_{-}\left(\eta_{1}, \eta_{2}\right)= & 2 \sqrt{\eta_{1} \eta_{2}}\left(\frac{\eta_{1}+\eta_{2}}{\eta_{1}-\eta_{2}} \ln \frac{\eta_{1}}{\eta_{2}}-2+\ln \left(\eta_{1} \eta_{2}\right)-\frac{\operatorname{div}}{2}\right) .
\end{aligned}
$$

The fermionic contribution to $S, \Delta S^{f}$, can be also computed following Ref. [196]:

$$
\begin{aligned}
\Delta S^{f}=-\frac{1}{\pi} \sum_{i j}\{ & \left(C_{L}^{i j} Y_{L}^{i j}+C_{R}^{i j} Y_{R}^{i j}\right)\left[-\frac{\operatorname{div}}{12}-\frac{5}{9}+\frac{\eta_{i}+\eta_{j}}{3}+\frac{\ln \left(\eta_{i} \eta_{j}\right)}{6}\right. \\
& \left.+\frac{\eta_{i}-1}{12} f\left(\eta_{i}, \eta_{i}\right)+\frac{\eta_{j}-1}{12} f\left(\eta_{j}, \eta_{j}\right)-\frac{\chi_{+}\left(\eta_{i}, \eta_{j}\right)}{2}\right] \\
& -\left(C_{L}^{i j} Y_{R}^{i j}+C_{R}^{i j} Y_{L}^{i j}\right)\left[2 \sqrt{\eta_{i} \eta_{j}}+\sqrt{\eta_{i} \eta_{j}} \frac{f\left(\eta_{i}, \eta_{i}\right)+f\left(\eta_{j}, \eta_{j}\right)}{4}\right. \\
& \left.\left.+\frac{\chi_{-}\left(\eta_{i}, \eta_{j}\right)}{2}\right]\right\}-\Delta S_{S M}^{f},
\end{aligned}
$$

In both equations above, $f\left(\eta_{1}, \eta_{2}\right)$ and $\chi_{ \pm}\left(\eta_{i}, \eta_{j}\right)$ are as defined in Ref. [196] and "div" stands for the divergent contributions typically appearing in dimensional regularization. The last term corresponds to the subtraction of the SM light (top and bottom) fermionic contributions ${ }^{\ddagger}$.

Finally, to analyze the effect on the $Z \bar{b}_{L} b_{L}$ coupling, we follow Ref. [195] for the computation of the corrections to the $g_{L}^{b}$ parameter defined in Eq. (9.4). Again, only the top and bottom sectors will be taken into account. As before, the mass generation mechanism for the lighter fermions is expected to have a lesser impact on EW precision tests. Moreover, the bottom quark mass will be neglected $\left(y_{1}^{\prime}=\right.$ $\left.y_{2}^{\prime}=0\right)^{\S}$. The fermion-gauge couplings relevant to this case are the $\mathrm{Z}$ couplings for the charge $2 / 3$ and $-1 / 3$ sectors which can be read from Eqs. (9.17) and (9.18), and the couplings to the $W^{ \pm}$boson between the RH $2 / 3$ and the $\mathrm{LH}-1 / 3$ sectors (see the matrix $\mathcal{V}_{L}^{\mathcal{T B}}$ defined after Eq. (9.20)). In addition to the $\mathrm{NC}$ and $\mathrm{CC}$ couplings in Eq. (9.15), the interactions of the charged longitudinal gauge boson components $\left(\pi^{ \pm}\right)$are needed:

$$
\mathcal{L}_{\pi^{ \pm}}=\bar{\Psi}^{\text {phys }} \frac{g}{\sqrt{2}}\left(W_{L} P_{L}+W_{R} P_{R}\right) \Psi^{\text {phys }} \pi^{+}+\text {h.c. }
$$

where

$$
W_{L}=R \mathcal{W}_{L} L^{\dagger}, \quad W_{R}=L \mathcal{W}_{R} R^{\dagger},
$$

$\ddagger$ The SM fermionic contributions to $S$ and $T$ with only one generation of quarks follow from Eq. (9.15) considering a two-component fermion field $\Psi^{S M}=(t, b)$, with $\mathcal{M}^{S M}=\operatorname{diag}\left(m_{t}, m_{b}\right)$ and coupling matrices $\mathcal{Q}^{S M}=Y_{R}^{S M}=\operatorname{diag}(+2 / 3,-1 / 3), C_{L}^{S M}=\operatorname{diag}(+1,-1), Y_{L}^{S M}=+\frac{1}{6} \mathbb{1}_{2 \times 2}$, $V_{L}^{S M}=\operatorname{antidiag}(0,1), C_{R}^{S M}=V_{R}^{S M}=\mathbf{0}_{2 \times 2}$.

$\S$ The cancellation of divergences in the computation of $\Delta g_{L}^{b}$ has been verified in this approximation. 


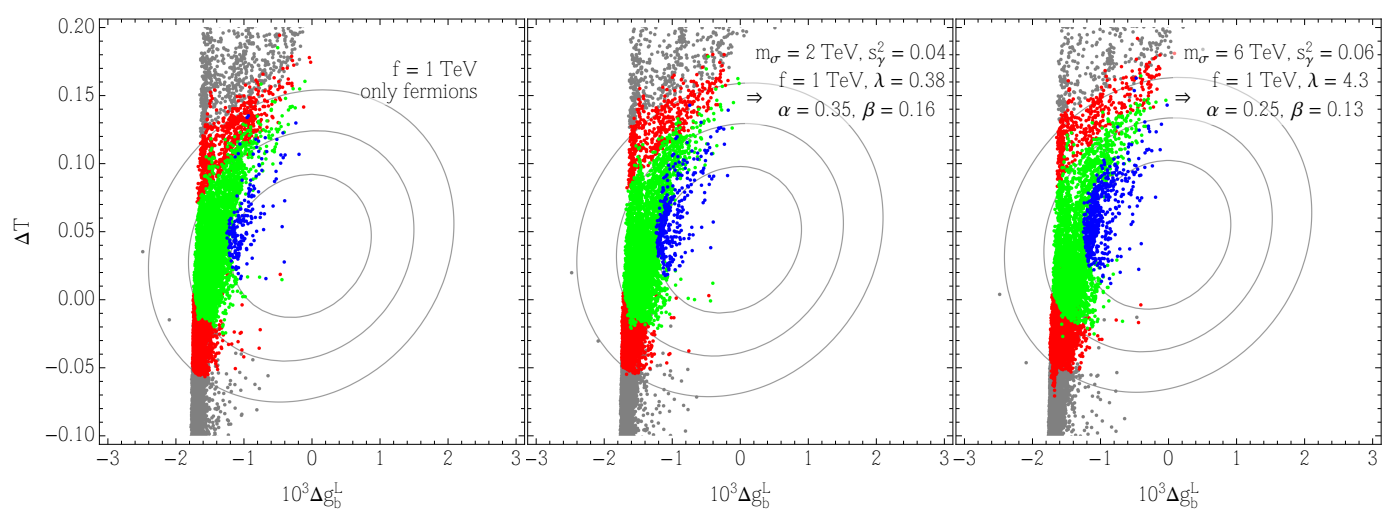

Figure 9.3: Scalar and fermionic impact on the $T$ parameter and on the $Z \bar{b}_{L} b_{L}$ coupling $g_{b}^{L}$.

with $\mathcal{W}_{L}$ and $\mathcal{W}_{R}$ being the mixing matrices in the interaction basis, given in the present model by

$$
\begin{aligned}
\mathcal{W}_{L ; R} & =\left(\begin{array}{cccc}
0 & \mathcal{W}_{L ; R}^{X^{u} \mathcal{T}} & \mathbf{0}_{1 \times 6} & 0 \\
\mathbf{0}_{6 \times 1} & \mathbf{0}_{6 \times 6} & \mathcal{W}_{L ; R}^{\mathcal{T}} & \mathbf{0}_{6 \times 1} \\
\mathbf{0}_{6 \times 1} & \mathbf{0}_{6 \times 6} & \mathbf{0}_{6 \times 6} & \mathcal{W}_{L ; R}^{\mathcal{B} X^{\prime d}} \\
0 & \mathbf{0}_{1 \times 6} & \mathbf{0}_{1 \times 6} & 0
\end{array}\right), \\
\mathcal{W}_{L ; R}^{X^{u} \mathcal{T}} & =\frac{\sqrt{2}}{g}\left(0,0,0,0,-y_{2} ;-y_{1}, 0\right) \\
\left(\mathcal{W}_{L ; R}^{\mathcal{B} X^{\prime d}}\right)^{\dagger} & =\frac{\sqrt{2}}{g}\left(0,0,0,0, y_{1}^{\prime} ; y_{2}^{\prime}, 0\right) .
\end{aligned}
$$

The $6 \times 6$ matrix $\mathcal{W}_{L}^{\mathcal{T} \mathcal{B}}$ in this equation has all elements null but for its $(5,6)$ and $(6,5)$ entries which take values $y_{1}$ and $-y_{2}^{\prime}$, respectively; while $\mathcal{W}_{R}^{\mathcal{T H}}$ is a $6 \times 6$ matrix of null elements but for its $(5,6)$ and $(6,5)$ entries which take values $y_{2}$ and $-y_{1}^{\prime}$, respectively. In practice, only the entries connecting -after rotation- the charge $2 / 3$ fermions to $b_{L}$ will enter the computation.

In the numerical analysis, we will consider for the scalar sector the two sets of values considered earlier to compute the impact from the heavy scalar: one within the perturbative regime $\left(m_{\sigma}=2 \mathrm{TeV}, s_{\gamma}^{2}=0.04\right)$, and the other roughly where perturbativity is lost $\left(m_{\sigma}=6 \mathrm{TeV}, s_{\gamma}^{2}=0.06\right)$. See discussion above.

The exotic fermionic masses will be allowed to vary randomly between $800 \mathrm{GeV}$ and $\mathcal{O}(10 \mathrm{TeV})$, as the heavy top partners with electric charges $+5 / 3$ and $+2 / 3$ are bounded to be above $800-1000 \mathrm{GeV}[197,198]$, depending on the dominant decay mode. In the light fermion sector, the top and bottom masses will be allowed to vary within the intervals $m_{t}=173 \pm 5 \mathrm{GeV}$ and $m_{b}=4.6 \pm 2 \mathrm{GeV}$, respectively, for illustrative purposes.

Figs. 9.1 to 9.4 depict the points that satisfy a $\chi^{2}$ global fit to the precision pseudoobservables $S, T$ and $\Delta g_{L}^{b}$, where the blue, green, and red points are the allowed $1 \sigma, 2 \sigma$ and $3 \sigma$ regions, respectively, while gray points lie above the $3 \sigma$ limit. The central values, uncertainties and correlation matrix are taken from Ref. [188]. The 
ellipses drawn in the $\Delta S-\Delta T$ plane in Figs. 9.1 and 9.2 are the projection for $\Delta g_{b}^{L}=0$, while those in the $\Delta T-\Delta g_{b}^{L}$ plane in Fig. 9.3 take $\Delta S \simeq \Delta S^{(h \text { and } \sigma)}$, the value coming only from the scalar sector. The latter is a good approximation since $S$ gets in practice a very small correction from the heavy fermions, see Fig. 9.2.

\section{$\Delta S$ versus $\Delta T$}

As shown in Fig. 9.2, the fermion sector can actually lead to large deviations in the value of the $T$ parameter. In the first panel of Fig. 9.2 only the fermionic contributions are depicted; while the last two panels show the fermion plus scalar combined results. As in Fig. 9.1, we can see that the lighter the $\sigma$ particle, the less tension follows with respect to EW precision data, in particular due to the impact on $\Delta T$, although it is to be noted that even for large $m_{\sigma}$ fermionic contributions could bring $(S, T)$ within the experimentally allowed region.

The sign of the fermionic contributions to $S$ and $T$ can be qualitatively understood in terms of the light-heavy fermion mixings and the mass hierarchy between the heavy eigenstates. For instance, large mixing values with a heavy singlet are known to induce large positive contributions to $\Delta T$, as pointed out in Ref. [195], as a result of the custodial symmetry being broken by the singlet-doublet mixing. This is illustrated in Ref. [199], by using a simple fermion setup. They consider heavy vector fermions coupling directly to both the light doublet $q_{L}$ and the light singlet $q_{R}$. When only a heavy singlet is present, the expected contributions to $\Delta T$ and $\Delta S$ are both significant (although the ones to $\Delta T$ are more important) and positive for the regime we consider, see their Eq. (32). Instead, when only a heavy vector doublet is taken into account, they obtain sizable contributions to $\Delta T$ and very small to $\Delta S$, with a sign proportional to the sign of the mass splitting between the heavy eigenstates with charge $2 / 3$ and $-1 / 3$.

It is not possible to apply those conclusions in Ref. [199] directly here, though, as in our setup the light fermion mass generation involves necessarily and simultaneously both a heavy doublet and a heavy singlet, see Eq. (8.38). Nevertheless, the fact that fermionic corrections to $\Delta S$ are found to be mainly positive is consistent with being dominated by the participation of a heavy singlet $\left(T_{1}\right)$. Fig. 9.4 shows that indeed a large mixing between $t_{L}$ and the singlet $T^{(1)}$ leads to a positive $\Delta T$ (left panel) while the negative corrections to $\Delta T$ obtained are consistent instead with a large mixing between $t_{R}$ and the doublet component $X^{d}$ (middle panel).

\section{$\Delta T$ versus $\Delta g_{L}^{b}$}

The deviations induced in the $Z b b$ coupling provide additional bounds: even if the model parameters do not impact on $\Delta g_{b}^{L}$ at tree level, the top partners may induce at loop level deviations from the SM value. Fig. 9.3 depicts the purely fermionic and the scalar plus fermion combined contributions in the $T-g_{L}^{b}$ parameter space. The right panel in Fig. 9.4 show a sizable and positive impact on $g_{L}^{b}$ of the mixing between $t_{L}$ and the charge $2 / 3$ heavy singlets $T^{(1)}$ and $T^{(5)}$, similarly to what is found in Ref. [195]. 

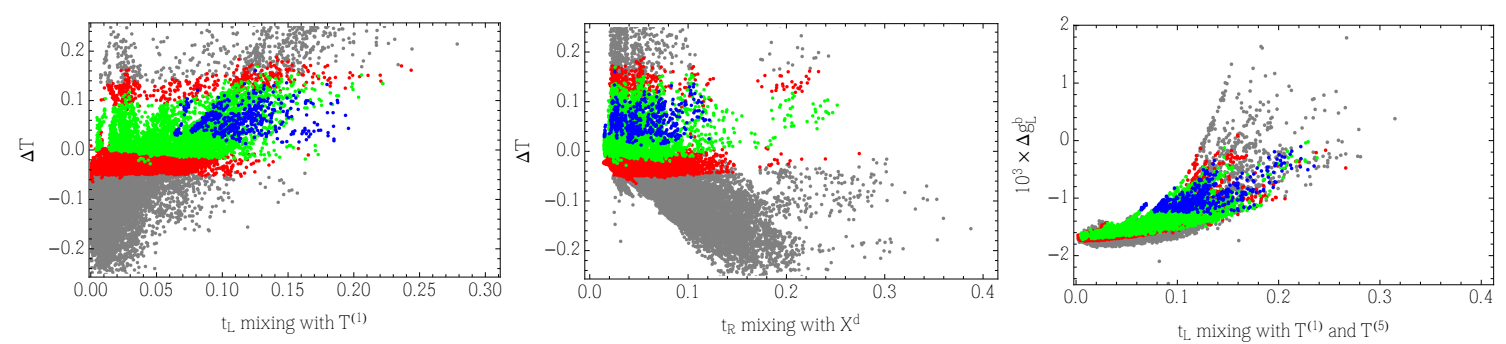

Figure 9.4: Examples of correlations between the size of different fermion mixings and electroweak precision measurements. The label " $t_{L, R}$ mixing with $\Psi_{1}, \Psi_{2}, \ldots$ " indicates $\left(\left|U_{1}^{L, R}\right|^{2}+\left|U_{2}^{L, R}\right|^{2}+\ldots\right)^{1 / 2}$, where $U^{L, R}$ denote the left or right rotation that diagonalizes the mass matrix.

As a final remark, there are considerable mixings in the fermion sector for which the dominant effects go schematically as $\tan \theta_{i j} \sim \Lambda_{i} / M_{j}$. It could be thus suspected that large deviations in the $W t b$ coupling should occur. However, since these rotations are mainly driven by the $S O(5)$ breaking couplings $\Lambda_{i}$ and $\Lambda_{i}^{\prime}$ in Eq. (8.36), which are custodial symmetry preserving, it is found that a rotation in the top sector is mostly compensated by a corresponding one in the bottom sector, leading to practically no deviation in $V_{t b}$.

\subsection{Higgs and $\sigma$ coupling to gluons}

This section and the next one deal with the scalar to photons and to gluons effective couplings, arising at one-loop level. The scalar-gluon-gluon amplitudes $h g g(\sigma g g)$ is defined as

$$
\mathcal{A}_{h(\sigma)} \equiv \mathcal{A}_{h(\sigma) \leftrightarrow g g}\left(m_{h(\sigma)}^{2}\right)=-i \frac{\alpha_{s}}{\pi} g_{h(\sigma)}\left(p \cdot k g^{\mu \nu}-p^{\mu} k^{\nu}\right) \delta^{a b},
$$

where $g_{h}$ and $g_{\sigma}$ are scale-dependent functions that parametrize the amplitude strength, $\alpha_{s}=g_{s}^{2} / 4 \pi$ with $g_{s}$ denoting the QCD coupling constant, $p$ and $k$ stand for the gluon four-momenta, and $a, b$ are color indices. In the case of the SM, the $h g g$ coupling is induced only at one loop level and the amplitude is dominated by the top quark:

$$
g_{h}^{S M}=\left(\frac{y_{t}}{\sqrt{2}}\right) \frac{1}{m_{t}} I\left(\frac{m_{h}^{2}}{m_{t}^{2}}\right),
$$

where $y_{t}$ is the top Yukawa coupling $\left(m_{t} \equiv y_{t} v / \sqrt{2}\right)$ and $I\left(m_{h}^{2} / m_{t}^{2}\right) / m_{t}$ is the loop factor with

$$
I\left(\frac{q^{2}}{m^{2}}\right)=\int_{0}^{1} d x \int_{0}^{1-x} d z \frac{1-4 x z}{1-x z \frac{q^{2}}{m^{2}}} \approx\left\{\begin{array}{ccc}
1 / 3 & \text { for } & m^{2} \gg q^{2} \\
0 & \text { for } & m^{2} \ll q^{2}
\end{array}\right\} .
$$

The SM bottom contribution corresponds to $I\left(m_{h}^{2} / m_{b}^{2}\right) \approx 10^{-2}$ and is thus usually neglected Expanding the global field-dependent mass matrix $\mathcal{M}(h, \sigma)$ in

IThe large mass limit in the integral is customarily applied for $m_{h}<2 m_{i}$, which includes the top case. 
Eqs. (8.41)-(8.44) around the scalar field vevs, $v$ and $v_{\sigma}$, and defining the following constant matrices

$$
\overline{\mathcal{M}} \equiv \mathcal{M}\left(v, v_{\sigma}\right),\left.\quad \frac{\partial \overline{\mathcal{M}}}{\partial h} \equiv \frac{\partial \mathcal{M}(h, \sigma)}{\partial h}\right|_{\substack{h=v \\ \sigma=v_{\sigma}}},\left.\quad \frac{\partial \overline{\mathcal{M}}}{\partial \sigma} \equiv \frac{\partial \mathcal{M}(h, \sigma)}{\partial \sigma}\right|_{\substack{h=v \\ \sigma=v_{\sigma}}}
$$

the fermionic mass Lagrangian Eq. (8.41) can be written as

$$
-\mathcal{L}_{Y}=\bar{\Psi}_{L} \overline{\mathcal{M}} \Psi_{R}+\hat{h} \bar{\Psi}_{L} \frac{\partial \overline{\mathcal{M}}}{\partial h} \Psi_{R}+\hat{\sigma} \bar{\Psi}_{L} \frac{\partial \overline{\mathcal{M}}}{\partial \sigma} \Psi_{R}+\text { h.c. }
$$

where $\hat{h}$ and $\hat{\sigma}$ are the unrotated scalar fluctuations, see Eq. (8.15). Performing the rotation to the fermionic mass eigenstate basis $\left\{\Psi_{i} \rightarrow \Psi_{i}^{\text {phys }}\right\}$ :

$$
(\overline{\mathcal{M}})_{i j} \rightarrow m_{i} \delta_{i j} \quad, \quad\left(\frac{\partial \overline{\mathcal{M}}}{\partial h}\right)_{i j} \rightarrow\left(Y_{h}\right)_{i j} \quad, \quad\left(\frac{\partial \overline{\mathcal{M}}}{\partial \sigma}\right)_{i j} \rightarrow\left(Y_{\sigma}\right)_{i j}
$$

where $m_{i}, Y_{h}$ and $Y_{\sigma}$ are respectively the masses and the couplings to the unrotated scalars fields $\hat{h}, \hat{\sigma}$ of the physical fermionic states $\|^{.}$

It is straightforward to obtain the physical $h \leftrightarrow g g$ and $\sigma \leftrightarrow g g$ amplitudes combining those involving the unrotated $\hat{h}$ and $\hat{\sigma}$ fields. The latter will require the substitution of the SM loop factor in Eq. (9.29) as follows,

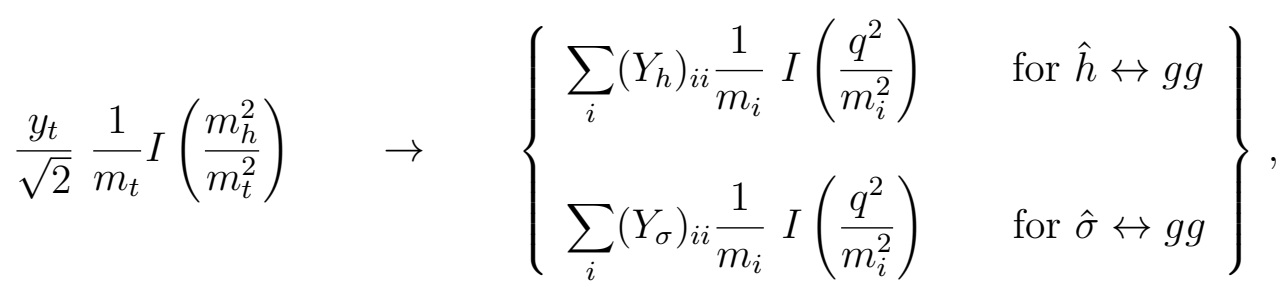

where $q^{2}=m_{h}^{2}$ for $h \leftrightarrow g g$ on-shell transitions, while $q^{2}=m_{\sigma}^{2}$ for $\sigma \leftrightarrow g g$ on-shell transitions, and where the sum runs over all colored fermion species present in the model.

\section{$h \leftrightarrow g g$ transitions}

If all fermion masses were much larger than $m_{h}$, it would be possible to simply factorize the constant integral outside the sum as follows:

$$
\sum_{i} \frac{\left(Y_{h}\right)_{i i}}{m_{i}} I\left(\frac{m_{h}^{2}}{m_{i}^{2}}\right) \approx \frac{1}{3} \sum_{i} \frac{\left(Y_{h}\right)_{i i}}{m_{i}}=\frac{1}{6} \frac{d}{d h} \log \operatorname{det}\left(\overline{\mathcal{M}} \overline{\mathcal{M}}^{\dagger}\right),
$$

where the last term is written in the original unrotated fermionic basis since trace and determinant are invariant under a change of basis. All fermions in the model are indeed much heavier than the Higgs particle but for the bottom quark, whose loop contribution $I\left(m_{h}^{2} / m_{b}^{2}\right)$ is actually negligible. Therefore, at energies $q^{2} \approx m_{h}^{2}$ we

\footnotetext{
"For instance, in this notation $\left(Y_{h}\right)_{t t}=y_{t} / \sqrt{2}$.
} 
should remove the contribution of the bottom quark included in Eq. (9.32), which results in the following effective couplings:

$$
\begin{aligned}
g_{\hat{h}}\left(m_{h}^{2}\right) \approx & \left.\frac{1}{6} \frac{d}{d h}\left(\log \operatorname{det}\left(\mathcal{M} \mathcal{M}^{\dagger}\right)-\log \left(m_{b}(h, \sigma) m_{b}^{*}(h, \sigma)\right)\right)\right|_{\substack{h=v \\
\sigma=v_{\sigma}}} \\
& =\frac{1}{3 v}+O\left(\frac{v}{M_{1}^{\prime} M_{5}^{\prime}}\right), \\
g_{\hat{\sigma}}\left(m_{h}^{2}\right) \approx & \left.\frac{1}{6} \frac{d}{d \sigma}\left(\log \operatorname{det}\left(\mathcal{M} \mathcal{M}^{\dagger}\right)-\log \left(m_{b}(h, \sigma) m_{b}^{*}(h, \sigma)\right)\right)\right|_{\substack{h=v \\
\sigma=v_{\sigma}}} \\
& =-\frac{y_{2}}{3 M_{5}} \frac{\Lambda_{2}}{\Lambda_{3}}+O\left(\frac{v_{\sigma}}{M_{1}^{\prime} M_{5}^{\prime}}, \frac{v_{\sigma}}{M_{5}^{2}}\right),
\end{aligned}
$$

where the eigenvalue of the field-dependent mass matrix corresponding to the bottom quark reads

$$
m_{b}(h, \sigma)=\frac{y_{1}^{\prime} \Lambda_{1}^{\prime} \Lambda_{3}^{\prime}-y_{1}^{\prime} y_{2}^{\prime} \Lambda_{1}^{\prime} \Lambda_{2}^{\prime} \sigma / M_{5}^{\prime}}{M_{1}^{\prime} M_{5}^{\prime}-y_{1}^{\prime} y_{2}^{\prime}\left(h^{2}+\sigma^{2}\right)} \frac{h}{\sqrt{2}} .
$$

The $h \leftrightarrow g g$ amplitude is then given by Eq. (9.27), with

$$
g_{h} \equiv g_{\hat{h}}\left(m_{h}^{2}\right) \cos \gamma-g_{\hat{\sigma}}\left(m_{h}^{2}\right) \sin \gamma
$$

\section{$\sigma \leftrightarrow g g$ transitions}

Following an analogous procedure to the one just explained, the $\sigma g g$ amplitude can be obtained using Eq. (9.31) for $q^{2}=m_{\sigma}^{2}$. The difference with the previous case is that we assume the $\sigma$ mass to be comparable or larger than the top quark mass, so its contribution should be also subtracted from that of the complete fermion mass matrix, just as the bottom quark was subtracted before. In other words, here $m_{b} \ll m_{t}, m_{\sigma} \ll m_{i}$, where $m_{i}$ denotes the heavy fermion masses. We then subtract the bottom contribution as before $\left(I\left(m_{\sigma}^{2} / m_{b}^{2}\right) \approx 0\right)$, but in case the $\sigma$ and top masses are comparable, we take into account the $q^{2}$ dependence in the top loop. This results in: 


$$
\begin{aligned}
& g_{\hat{h}}\left(m_{\sigma}^{2}\right) \approx \frac{1}{6} \frac{d}{d h}\left(\log \operatorname{det}\left(\mathcal{M} \mathcal{M}^{\dagger}\right)-\log \left(m_{t}(h, \sigma) m_{t}^{*}(h, \sigma)\right)\right. \\
&\left.-\log \left(m_{b}(h, \sigma) m_{b}^{*}(h, \sigma)\right)\right)\left.\right|_{\substack{h=v \\
\sigma=v_{\sigma}}}+\frac{1}{v} I\left(\frac{m_{\sigma}^{2}}{m_{t}^{2}}\right) \\
&= \frac{1}{v} I\left(\frac{m_{\sigma}^{2}}{m_{t}^{2}}\right)-\frac{2}{3} v\left(\frac{y_{1} y_{2}}{M_{1} M_{5}}+\frac{y_{1}^{\prime} y_{2}^{\prime}}{M_{1}^{\prime} M_{5}^{\prime}}\right)+O\left(\frac{v v_{\sigma}^{2}}{M_{1}^{2} M_{5}^{2}}, \frac{v v_{\sigma}^{2}}{M^{\prime 2} M_{1}^{\prime 2}}\right) \\
& g_{\hat{\sigma}}\left(m_{\sigma}^{2}\right) \approx \frac{1}{6} \frac{d}{d \sigma}\left(\log \operatorname{det}\left(\mathcal{M} \mathcal{M}^{\dagger}\right)-\log \left(m_{t}(h, \sigma) m_{t}^{*}(h, \sigma)\right)\right. \\
&\left.\quad-\log \left(m_{b}(h, \sigma) m_{b}^{*}(h, \sigma)\right)\right)\left.\right|_{\substack{h=v \\
\sigma=v_{\sigma}}}-\frac{y_{2}}{M_{5}} \frac{\Lambda_{2}}{\Lambda_{3}} I\left(\frac{m_{\sigma}^{2}}{m_{t}^{2}}\right) \\
&=-\frac{y_{2}}{M_{5}} \frac{\Lambda_{2}}{\Lambda_{3}} I\left(\frac{m_{\sigma}^{2}}{m_{t}^{2}}\right)-\frac{2}{3} v_{\sigma}\left(\frac{y_{1} y_{2}}{M_{1} M_{5}}+\frac{y_{1}^{\prime} y_{2}^{\prime}}{M_{1}^{\prime} M_{5}^{\prime}}\right) \\
&+O\left(\frac{v_{\sigma}^{3}}{M_{1}^{2} M_{5}^{2}}, \frac{v_{\sigma}^{3}}{M_{1}^{\prime 2} M_{5}^{\prime 2}}\right),
\end{aligned}
$$

where the eigenvalue of the field-dependent mass matrix corresponding to the top quark reads

$$
m_{t}(h, \sigma)=\frac{y_{1} \Lambda_{1} \Lambda_{3}-y_{1} y_{2} \Lambda_{1} \Lambda_{2} \sigma / M_{5}}{M_{1} M_{5}-y_{1} y_{2}\left(h^{2}+\sigma^{2}\right)} \frac{h}{\sqrt{2}} .
$$

The $\sigma \leftrightarrow g g$ amplitude is finally given by Eqs. (9.27) and (9.36), with

$$
g_{\sigma} \equiv g_{\hat{h}}\left(m_{\sigma}^{2}\right) \sin \gamma+g_{\hat{\sigma}}\left(m_{\sigma}^{2}\right) \cos \gamma .
$$

The moduli of the squared matrix elements for $g g \rightarrow h$ and $g g \rightarrow \sigma$, averaged over the polarizations of the initial state, are then given by

$$
\begin{aligned}
& \overline{\left|\mathcal{A}_{h}\right|^{2}}=\frac{\alpha_{S}^{2} m_{h}^{4}}{64 \pi^{2}} g_{h}^{2}, \\
& \overline{\left|\mathcal{A}_{\sigma}\right|^{2}}=\frac{\alpha_{S}^{2} m_{\sigma}^{4}}{64 \pi^{2}} g_{\sigma}^{2} .
\end{aligned}
$$

In terms of these amplitudes, the cross section at the parton level can be expressed as

$$
\sigma_{\text {part }}(g g \rightarrow h)=\overline{\left|\mathcal{A}_{h}\right|^{2}} \frac{\pi}{s_{\text {part }}} \delta\left(s_{\text {part }}-m_{h}^{2}\right)=\overline{\left|\mathcal{A}_{h}\right|^{2}} \frac{\pi}{\tau s^{2}} \delta\left(\tau-\frac{m_{h}^{2}}{s}\right),
$$

where as usual $s_{\text {part }}$ denotes the center-of-mass energy at the parton level $s_{\text {part }}=\tau s$. A similar expression holds for $\sigma_{\text {part }}(g g \rightarrow \sigma)$. By convoluting the cross-section with the gluon densities $G(x)$, we finally obtain

$$
\begin{aligned}
& \sigma(p p \rightarrow h)=\overline{\left|\mathcal{A}_{h}\right|^{2}} \frac{\pi}{m_{h}^{2} s} \int_{m_{h}^{2} / s}^{1} \frac{d x}{x} G(x) G\left(\frac{m_{h}^{2}}{s x}\right), \\
& \sigma(p p \rightarrow \sigma)=\overline{\left|\mathcal{A}_{\sigma}\right|^{2}} \frac{\pi}{m_{\sigma}^{2} s} \int_{m_{\sigma}^{2} / s}^{1} \frac{d x}{x} G(x) G\left(\frac{m_{\sigma}^{2}}{s x}\right) .
\end{aligned}
$$


In summary, for very heavy fermion partners the $h$-gluon-gluon transitions are dominated by the top quark contribution, see Eq. (9.33); while they might have a more significant impact on $\sigma$-gluon-gluon transitions, see Eq. (9.36).

\subsection{Higgs and $\sigma$ decay into $\gamma \gamma$}

There are no direct $h \gamma \gamma$ or $\sigma \gamma \gamma$ couplings in our Lagrangian; but they arise instead as effective interactions from loops of fermions and, in the case of $h \gamma \gamma$, also of massive vector bosons. In order to compute the effective couplings, we will use a similar procedure to the one followed in the previous section. Let the scalar-photon-photon amplitudes $h \gamma \gamma$ and $\sigma \gamma \gamma$ be defined as

$$
\mathcal{A}_{h(\sigma) \leftrightarrow \gamma \gamma}\left(m_{h(\sigma)}^{2}\right)=i \frac{\alpha}{\pi} \Omega_{h(\sigma)}\left(p \cdot k g^{\mu \nu}-p^{\mu} k^{\nu}\right) \delta^{a b},
$$

where $\Omega_{h}$ and $\Omega_{\sigma}$ are scale-dependent functions. The decay amplitudes are then given by

$$
\Gamma(h \rightarrow \gamma \gamma)=\frac{\alpha^{2} m_{h}^{3}}{64 \pi^{3}}\left|\Omega_{h}\right|^{2}, \quad \Gamma(\sigma \rightarrow \gamma \gamma)=\frac{\alpha^{2} m_{\sigma}^{3}}{64 \pi^{3}}\left|\Omega_{\sigma}\right|^{2} .
$$

The contributions can again be decomposed as

$$
\begin{aligned}
& \Omega_{h}=\cos \gamma \Omega_{\hat{h}}\left(m_{h}^{2}\right)-\sin \gamma \Omega_{\hat{\sigma}}\left(m_{h}^{2}\right), \\
& \Omega_{\sigma}=\sin \gamma \Omega_{\hat{h}}\left(m_{\sigma}^{2}\right)+\cos \gamma \Omega_{\hat{\sigma}}\left(m_{\sigma}^{2}\right) .
\end{aligned}
$$

The unrotated sigma field $\hat{\sigma}$ only couples to fermions; while $\hat{h}$ couples both to fermions and the EW gauge bosons. In this case this means $\hat{h}$ has a contribution for the $W$ boson besides that of fermion fields:

$$
\Omega_{\hat{h}}\left(q^{2}\right)=\Omega_{\hat{h}}^{F}\left(q^{2}\right)+\Omega_{\hat{h}}^{W}\left(q^{2}\right), \quad \Omega_{\hat{\sigma}}\left(q^{2}\right)=\Omega_{\hat{\sigma}}^{F}\left(q^{2}\right),
$$

where the superscripts $F$ and $W$ stand for fermionic and gauge contributions, respectively. The latter is analogous to the SM one, that is,

$$
\Omega_{\hat{h}}^{W}\left(q^{2}\right)=\frac{g^{2} v}{8 m_{W}^{2}} I_{W}\left(\frac{4 m_{W}^{2}}{q^{2}}\right)
$$

where the factor $g^{2} v$ results from the Higgs $-W W$ vertex, and the remaining part, $I_{W} / 8 m_{W}^{2}$, results from the kinematics of the loop integral:

$$
I_{W}(x)=2+3 x+3 x(2-x) f(x), \quad f(x)=\left\{\begin{array}{ll}
\arcsin ^{2}(1 / \sqrt{x}) & x \geq 1 \\
-\frac{1}{4}\left[\log \frac{1+\sqrt{1-x}}{1-\sqrt{1-x}}-i \pi\right]^{2} & x<1
\end{array} .\right.
$$

\section{$h \leftrightarrow \gamma \gamma$ transitions}

At the Higgs mass scale, the SM $\hat{h} W W$ coupling in Eq. (9.46) is given by

$$
\Omega_{\hat{h}}^{W}\left(m_{h}^{2}\right) \approx \frac{4.2}{v}
$$


while the SM quark contributions are given by

$$
\Omega_{h}^{S M, F}=-6 \sum_{f} Q_{f}^{2}\left(\frac{y_{f}}{\sqrt{2}}\right) \frac{1}{m_{f}} I\left(\frac{m_{h}^{2}}{m_{f}^{2}}\right) \approx-\frac{8}{9} \frac{1}{v},
$$

where $y_{f}$ is the fermion Yukawa coupling, $m_{f}=y_{f} v / \sqrt{2}$, and the remaining factor $I / m_{f}$ results from the loop integral. The last expression in Eq. (9.49) corresponds to the top contribution, which dominates the SM fermionic contribution. The SM $h \rightarrow \gamma \gamma$ decay rate has an expression similar to what is given in Eq. (9.42) with $\Omega_{h}=\Omega_{h}^{S M, W}+\Omega_{h}^{S M, F}$. In the model under consideration these expressions for the quark contributions to the $h \rightarrow \gamma \gamma$ transitions are generalized as follows, in analogy with the $g g$ fusion analysis above:

$$
\begin{aligned}
& \Omega_{\hat{h}}^{F}\left(m_{h}^{2}\right)=-2 \sum_{f} N_{C}^{f} Q_{f}^{2} \omega_{f}^{h}\left(m_{h}^{2}\right)=-2\left[3\left(\frac{2}{3}\right)^{2} \omega_{2 / 3}^{h}\left(m_{h}^{2}\right)+3\left(-\frac{1}{3}\right)^{2} \omega_{-1 / 3}^{h}\left(m_{h}^{2}\right)\right], \\
& \Omega_{\hat{\sigma}}^{F}\left(m_{h}^{2}\right)=-2 \sum_{f} N_{C}^{f} Q_{f}^{2} \omega_{f}^{\sigma}\left(m_{h}^{2}\right)=-2\left[3\left(\frac{2}{3}\right)^{2} \omega_{2 / 3}^{\sigma}\left(m_{h}^{2}\right)+3\left(-\frac{1}{3}\right)^{2} \omega_{-1 / 3}^{\sigma}\left(m_{h}^{2}\right)\right],
\end{aligned}
$$

where $N_{C}^{f}$ is the number of colors of a given quark species $f$, and $\omega_{f}^{h}$ are scaledependent functions, which for charge $2 / 3$ and $-1 / 3$ fermions read

$$
\begin{aligned}
\omega_{2 / 3}^{h}\left(m_{h}^{2}\right) & \left.\equiv \frac{1}{6} \frac{d}{d h}\left(\log \operatorname{det}\left(\mathcal{M}_{\mathcal{T}} \mathcal{M}_{\mathcal{T}}^{\dagger}\right)\right)\right|_{\substack{h=v \\
\sigma=v_{\sigma}}}=\frac{1}{3 v} \\
\omega_{-1 / 3}^{h}\left(m_{h}^{2}\right) & \left.\equiv \frac{1}{6} \frac{d}{d h}\left(\log \operatorname{det}\left(\mathcal{M}_{\mathcal{B}} \mathcal{M}_{\mathcal{B}}^{\dagger}\right)-\log \left(m_{b}(h, \sigma) m_{b}^{*}(h, \sigma)\right)\right)\right|_{\substack { h=v \\
\sigma=v_{\sigma} \\
\begin{subarray}{c}{h \\
\text { s. }{ h = v \\
\sigma = v _ { \sigma } \\
\begin{subarray} { c } { h \\
\text { s. } } }\end{subarray}} \\
& =-\frac{2}{3} v \frac{y_{1}^{\prime} y_{2}^{\prime}}{M_{1}^{\prime} M_{5}^{\prime}}+\mathcal{O}\left(\frac{v v_{\sigma}^{2}}{M^{\prime 2} M^{\prime 2}}\right)
\end{aligned}
$$

As for the $\sigma$ particle, we find:

$$
\begin{aligned}
\omega_{2 / 3}^{\sigma}\left(m_{h}^{2}\right) & \left.\equiv \frac{1}{6} \frac{d}{d \sigma}\left(\log \operatorname{det}\left(\mathcal{M}_{\mathcal{T}} \mathcal{M}_{\mathcal{T}}^{\dagger}\right)\right)\right|_{\substack{h=v \\
\sigma=v_{\sigma}}}=-\frac{y_{2}}{3 M_{5}} \frac{\Lambda_{2}}{\Lambda_{3}}+\mathcal{O}\left(\frac{v_{\sigma}}{M_{5}^{2}}\right) \\
\omega_{-1 / 3}^{\sigma}\left(m_{h}^{2}\right) & \left.\equiv \frac{1}{6} \frac{d}{d \sigma}\left(\log \operatorname{det}\left(\mathcal{M}_{\mathcal{B}} \mathcal{M}_{\mathcal{B}}^{\dagger}\right)-\log \left(m_{b}(h, \sigma) m_{b}^{*}(h, \sigma)\right)\right)\right|_{\substack{h=v \\
\sigma=v_{\sigma}}} \\
& =-\frac{2}{3} \frac{v_{\sigma} y_{1}^{\prime} y_{2}^{\prime}}{M_{1}^{\prime} M_{5}^{\prime}}+\mathcal{O}\left(\frac{v_{\sigma}^{3}}{M_{1}^{\prime 2} M_{5}^{\prime 2}}\right) .
\end{aligned}
$$

As in the previous section, the bottom contribution has been neglected, while it has been assumed $m_{h} \ll m_{i}$ for the top mass and all other exotic fermion masses. 


\section{$\sigma \leftrightarrow \gamma \gamma$ transitions}

Similarly, for $\sigma$ decaying into two photons the contributions for the unrotated field $\hat{\sigma}$ are given by

$$
\begin{aligned}
& \Omega_{\hat{\sigma}}^{F}\left(m_{\sigma}^{2}\right)=-2 \sum_{f} N_{C}^{f} Q_{f}^{2} \omega_{f}^{\sigma}\left(m_{\sigma}^{2}\right)=-2\left[3\left(\frac{2}{3}\right)^{2} \omega_{2 / 3}^{\sigma}\left(m_{\sigma}^{2}\right)+3\left(-\frac{1}{3}\right)^{2} \omega_{-1 / 3}^{\sigma}\left(m_{\sigma}^{2}\right)\right], \\
& \Omega_{\hat{h}}^{F}\left(m_{\sigma}^{2}\right)=-2 \sum_{f} N_{C}^{f} Q_{f}^{2} \omega_{f}^{h}\left(m_{\sigma}^{2}\right)=-2\left[3\left(\frac{2}{3}\right)^{2} \omega_{2 / 3}^{h}\left(m_{\sigma}^{2}\right)+3\left(-\frac{1}{3}\right)^{2} \omega_{-1 / 3}^{h}\left(m_{\sigma}^{2}\right)\right],
\end{aligned}
$$

where

$$
\begin{aligned}
\omega_{2 / 3}^{\sigma}\left(m_{\sigma}^{2}\right) \equiv & \left.\frac{1}{6} \frac{d}{d \sigma}\left(\log \operatorname{det}\left(\mathcal{M}_{\mathcal{T}} \mathcal{M}_{\mathcal{T}}^{\dagger}\right)-\log \left(m_{t}(h, \sigma) m_{t}^{*}(h, \sigma)\right)\right)\right|_{\substack { h=v \\
\sigma=v_{\sigma} \\
\begin{subarray}{c}{h \\
M_{5}{ h = v \\
\sigma = v _ { \sigma } \\
\begin{subarray} { c } { h \\
M _ { 5 } } }\end{subarray}} \frac{y_{2}}{\Lambda_{3}} I\left(\frac{m_{\sigma}^{2}}{m_{t}^{2}}\right) \\
= & -\frac{y_{2}}{M_{5}} \frac{\Lambda_{2}}{\Lambda_{3}} I\left(\frac{m_{\sigma}^{2}}{m_{t}^{2}}\right)-\frac{2}{3} v_{\sigma} \frac{y_{1} y_{2}}{M_{1} M_{5}}+\mathcal{O}\left(\frac{v_{\sigma}^{3}}{M_{1}^{2} M_{5}^{2}}\right), \\
\omega_{-1 / 3}^{\sigma}\left(m_{\sigma}^{2}\right) \equiv & \left.\frac{1}{6} \frac{d}{d \sigma}\left(\log \operatorname{det}\left(\mathcal{M}_{\mathcal{B}} \mathcal{M}_{\mathcal{B}}^{\dagger}\right)-\log \left(m_{b}(h, \sigma) m_{b}^{*}(h, \sigma)\right)\right)\right|_{\substack{h=v \\
\sigma=v_{\sigma} \\
=}}-\frac{2}{3} v_{\sigma} \frac{y_{1}^{\prime} y_{2}^{\prime}}{M_{1}^{\prime} M_{5}^{\prime}}+\mathcal{O}\left(\frac{v_{\sigma}^{3}}{M_{1}^{\prime 2} M_{5}^{\prime 2}}\right),
\end{aligned}
$$

while for $\omega^{h}\left(m_{\sigma}^{2}\right)$ it results

$$
\begin{aligned}
\omega_{2 / 3}^{h}\left(m_{\sigma}^{2}\right) \equiv & \left.\frac{1}{6} \frac{d}{d h}\left(\log \operatorname{det}\left(\mathcal{M}_{\mathcal{T}} \mathcal{M}_{\mathcal{T}}^{\dagger}\right)-\log \left(m_{t}(h, \sigma) m_{t}^{*}(h, \sigma)\right)\right)\right|_{\substack { h=v \\
\sigma=v_{\sigma} \\
\begin{subarray}{c}{h \\
v{ h = v \\
\sigma = v _ { \sigma } \\
\begin{subarray} { c } { h \\
v } }\end{subarray}} \\
& +\frac{1}{v} I\left(\frac{m_{\sigma}^{2}}{m_{t}^{2}}\right) \\
= & \frac{1}{v} I\left(\frac{m_{\sigma}^{2}}{m_{t}^{2}}\right)-\frac{2}{3} v \frac{y_{1} y_{2}}{M_{1} M_{5}}+\mathcal{O}\left(\frac{v v_{\sigma}^{2}}{M_{1}^{2} M_{5}^{2}}\right), \\
\omega_{-1 / 3}^{h}\left(m_{\sigma}^{2}\right) \equiv & \left.\frac{1}{6} \frac{d}{d h}\left(\log \operatorname{det}\left(\mathcal{M}_{\mathcal{B}} \mathcal{M}_{\mathcal{B}}^{\dagger}\right)-\log \left(m_{b}(h, \sigma) m_{b}^{*}(h, \sigma)\right)\right)\right|_{\substack{h=v \\
\sigma=v_{\sigma} \\
=}} \\
= & \frac{2}{3} v \frac{y_{1}^{\prime} y_{2}^{\prime}}{M_{1}^{\prime} M_{5}^{\prime}}+\mathcal{O}\left(\frac{v v_{\sigma}^{2}}{M_{1}^{\prime 2} M_{5}^{\prime 2}}\right),
\end{aligned}
$$

where again it has been assumed that $m_{b} \ll m_{\sigma}, m_{t}$ comparable or lighter than the sigma mass, and $m_{\sigma} \ll m_{i}$ for all the other heavy quarks. Finally, the physical $h$ and $\sigma$ decay widths into two photons are given by

$$
\begin{aligned}
\Gamma(h \rightarrow \gamma \gamma) & =\frac{\alpha^{2} m_{h}^{3}}{64 \pi^{3}}\left|\cos \gamma\left[\Omega_{\hat{h}}^{W}\left(m_{h}^{2}\right)+\Omega_{\hat{h}}^{F}\left(m_{h}^{2}\right)\right]-\sin \gamma \Omega_{\hat{\sigma}}^{F}\left(m_{h}^{2}\right)\right|^{2}, \\
\Gamma(\sigma \rightarrow \gamma \gamma) & =\frac{\alpha^{2} m_{\sigma}^{3}}{64 \pi^{3}}\left|\sin \gamma\left[\Omega_{\hat{h}}^{W}\left(m_{\sigma}^{2}\right)+\Omega_{\hat{h}}^{F}\left(m_{\sigma}^{2}\right)\right]+\cos \gamma \Omega_{\hat{\sigma}}^{F}\left(m_{\sigma}^{2}\right)\right|^{2}
\end{aligned}
$$


In summary, $\sigma \rightarrow \gamma \gamma$ transitions are dominated by the $W^{ \pm}$loop contributions unless the scalar mixing is small enough for the heavy partner loop contribution to be significant.

\subsection{The $\sigma$ resonance at the $\mathrm{LHC}$}

This section explores what LHC data can say about a $\sigma$ resonance. ${ }^{* *}$ The constraints from electroweak precision tests analyzed in Sect. 9.2 showed that a scenario with a light $\sigma$ particle tends to diminish the tension with data. On the other side, naturalness prefers a sizable mass for a pGB Higgs and an unprotected $\sigma$ particle. Furthermore, the pGB interpretation implies the existence of a non-zero mixing between $\sigma$ and $h, \sin ^{2} \gamma \sim \xi \ll 1$ which would require a strong fine-tuning of the theory for a light sigma, see Eq. (9.1) and the discussion after Eqs. (8.30), (8.31).

In order to estimate the LHC constraints on the model, we recast many LHC searches for scalar resonances into the $\sigma$ parameter space, calculating the production cross section and decays of the $\sigma$ particle. The production of the $\sigma$ particle at the LHC may proceed mainly via two processes, gluon fusion and vector boson fusion (VBF). Gluon fusion usually dominates the production due to the large gluon parton distribution functions. Nevertheless, this conclusion is somewhat model-dependent as the heavy fermion couplings to $\sigma$ may a priori enhance or diminish the cross section. VBF depends essentially on the mixing angle $\gamma$, but it typically yields a lower production cross section than gluon fusion for $m_{\sigma}<\mathcal{O}(1 \mathrm{TeV})$. For this reason, we will assume the $\sigma$ is produced through gluon fusion in what follows.

To account for higher order corrections to $\Gamma(\sigma \rightarrow g g)$, we will profit from the results in the literature for a heavy SM-like Higgs boson $H^{\prime}$, using the following approximation

$$
\sigma(g g \rightarrow \sigma) \simeq \frac{|A(\sigma \rightarrow g g)|^{2}}{\left|A_{S M}\left(H^{\prime} \rightarrow g g\right)\right|^{2}} \sigma_{S M}\left(g g \rightarrow H^{\prime}\right),
$$

where $A\left(\sigma, H^{\prime} \rightarrow g g\right)$ refers to LO (one-loop) amplitudes and $\sigma_{S M}\left(g g \rightarrow H^{\prime}\right)$ is the NNLO standard gluon fusion production cross section given in Ref. [53].

Let us now discuss the LHC impact of the $\sigma$ particle by first considering an scenario in which the impact of the heavy fermions on gluon fusion is negligible as compared to the top contribution coming from the mixing with the Higgs. Then the production amplitude can be approximated by the top loop contribution for a heavy SM Higgs weighed down by $\sin \gamma$. The left panel in Fig. 9.5 shows the results from recasting different LHC searches for a heavy Higgs-like particle into constraints in the $\left\{m_{\sigma}, \sin ^{2} \gamma\right\}$ plane under this assumption.

The searches taken into account here include diphoton [201,202], diboson [203-206] and dihiggs $[207,208]$ decays. The constraint from Higgs measurements on the

${ }^{* *}$ Later work also explores other interesting phenomenological consequences for a scalar like the sigma particle, as Ref. [200] where the decay into fermions is studied. 

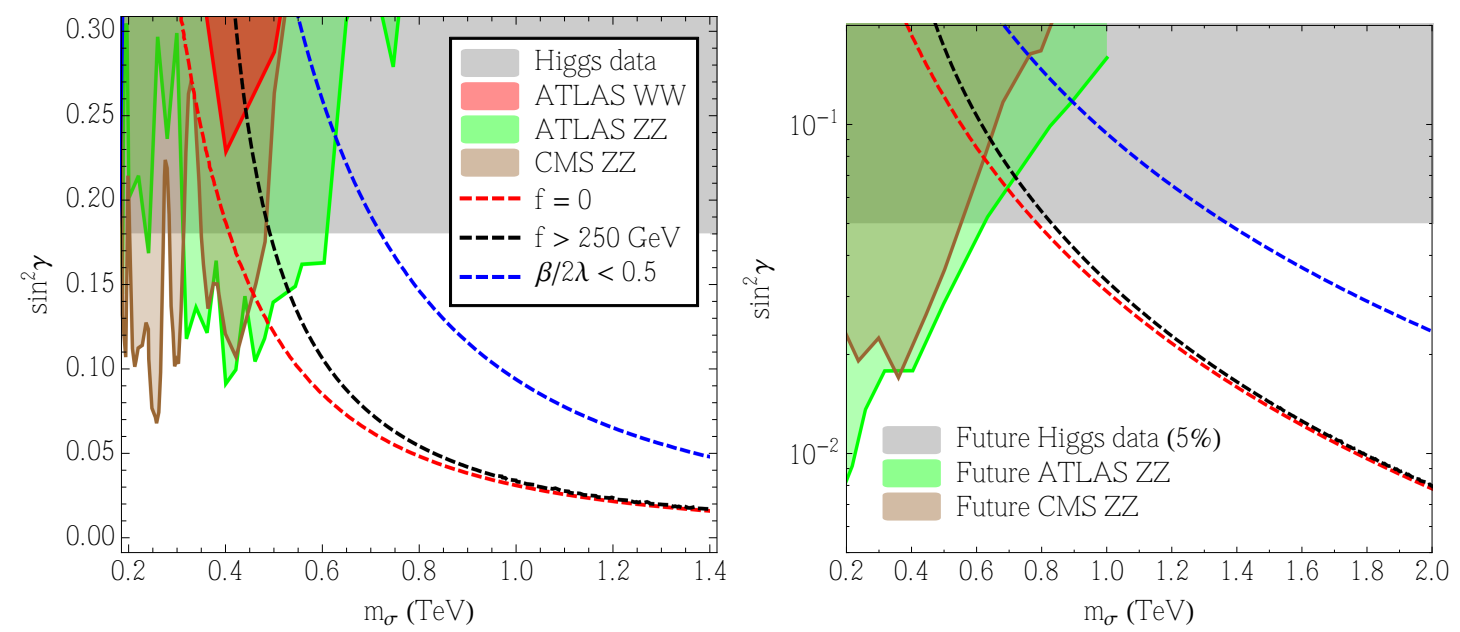

Figure 9.5: Constraints on $\sin ^{2} \gamma$ versus $\sigma$ mass parameter space from LHC run-1 (left panel) and prospects for LHC run-2 with an integrated luminosity of $3000 \mathrm{fb}^{-1}$ (right panel), considering gluon fusion is dominated by the top loop.

mixing $\sin ^{2} \gamma<0.18$ from Sect. 9.1, which is independent of $m_{\sigma}$, is also shown as a grey colored excluded area. Fig. 9.5 shows that run-1 LHC data were already sensitive to $m_{\sigma}<600 \mathrm{GeV}$ for $\sin ^{2} \gamma \simeq 0.1$. It is worth noting that these bounds apply to any generic scenario in which the Higgs particle is substituted by a Higgssinglet scalar system with a mixing angle $\gamma$, independently of the details of the theory. Now, considering the pGB Higgs theoretical motivation for the $S O(5) / S O(4)$ model discussed here, interesting bounds can be derived, since a pGB nature for the Higgs boson corresponds to the area to the right of the red curve depicted, see also Fig. 8.2. This curve corresponds to the minimal theoretical requirement $f^{2}>0$ for $S O(5)$ to be spontaneously broken, which results in a bound $m_{\sigma}>500 \mathrm{GeV}$, in particular from the impact of ATLAS $H_{\text {heavy }} \rightarrow Z Z$ searches. If $f^{2}$ values above the electroweak scale are instead required (black curve) $m_{\sigma}>550 \mathrm{GeV}$ follows.

The future prospects for this scenario are depicted on the right panel of Fig. 9.5. It shows the future LHC sensitivity in the $Z Z$ decay channel of the $14 \mathrm{TeV}$ LHC run with an integrated luminosity of $3 \mathrm{ab}^{-1}$, for both ATLAS and CMS [209]. It also shows the expectation for the excluded region for the scalar mixing from Higgs data, assuming a $5 \%$ precision on the Higgs couplings to SM particles. In conclusion, in the absence of any beyond the SM signal, future LHC data together with the theoretically motivated constraints mentioned above, could push the limit on the $\sigma$ mass above $900 \mathrm{GeV}-1.4 \mathrm{TeV}$.

The difference between the LHC predictions of the model discussed in this paper and those stemming from extending the SM by a generic scalar singlet (see e.g. Ref. [210]) is the underlying $S O(5)$ structure of the former, which prescribes a specific relation between the quartic terms in the potential as well as specific soft breaking terms. In the generic extra singlet scenarios, the allowed parameter space is given by the entire white area in Fig. 9.5, while a PNGB nature for the Higgs restricts the allowed region to the area to the right of the curves depicted in the figure.

Finally, let us discuss the possible impact from the exotic heavy fermions of the 

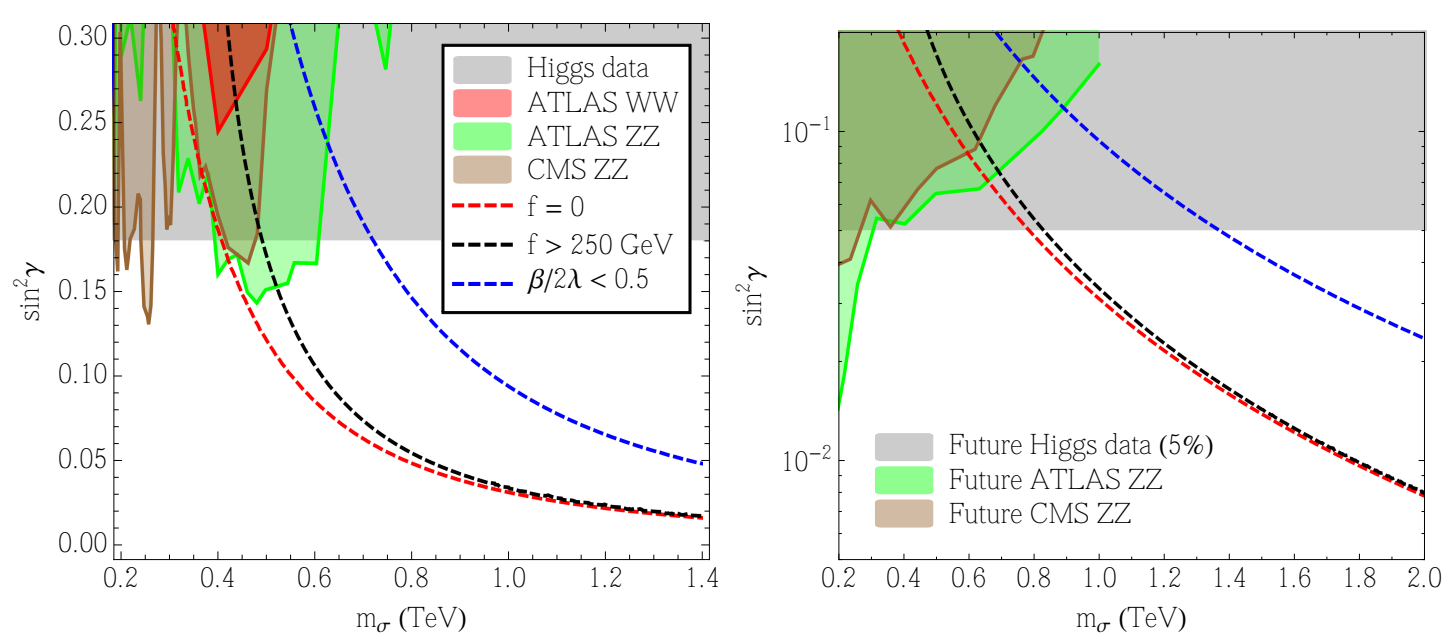

Figure 9.6: Constraints on $\sin ^{2} \gamma$ versus $\sigma$ mass parameter space from LHC run-1 (left panel) and prospects for LHC run-2 with an integrated luminosity of $3000 \mathrm{fb}^{-1}$ (right panel), considering a sizable contribution of the heavy fermion sector to gluon fusion. See text for details.

model. We use the approximate expressions in Eqs. (9.39) and (9.41), and assuming that the factor $y_{1} y_{2} / M_{1} M_{5}$ in Eq. (9.36) is the largest contribution. The results obtained are depicted in Fig. 9.6. It shows that the LHC bounds on $\sin ^{2} \gamma$ can be weakened by $\mathcal{O}(30-50 \%)$ with respect to the bounds found in Fig. 9.5. This is due to a destructive interference between the heavy fermions and the top loops, for the set of parameters considered. Moreover, future searches will be much more sensitive to the heavy fermion sector as they probe smaller mixing angles, thus entering regions in parameter space where the top quark is relatively less important to the $\sigma$ phenomenology. 


\section{Chapter 10}

\section{Connection with EFTs}

The analysis presented here [211] takes the model described in Chapter 8 and explores the limit $\lambda \rightarrow \infty$. This corresponds to a very heavy $\sigma$ particle. Indeed, sending its mass to infinity should make us fall onto the usual effective non-linear construction.

The number of couplings is usually very large in the most general case for the HEFT Lagrangian [125-127,140]. Therefore, the purpose of this chapter is to obtain the benchmark Lagrangian for models based on an $S O(5) / S O(4)$ breaking. See also Ref. [212].

Ref. [213] established a purely bosonic basis for a generic $S O(5) / S O(4)$ construction (among other patterns) with no $S O(5)$ explicit breaking. In this computation we find a reduced subset of those operators in the particular case of our minimal $S O(5)$ linear sigma model. We expect those operators to be common to the non-linear limit of any construction containing the $S O(5) / S O(4)$ spontaneous breaking. The leading order operators from the explicit $S O(5)$-breaking are also determined in this work.

Furthermore, the leading contributions from BSM heavy fermions are presented as well. However, instead of restricting the treatment to the very particular, rather model-dependent, setup of heavy fermions described in previous chapters, a simplified effective Yukawa coupling is defined to handle the dominant fermion-induced effects (see section 10.1). In this fashion, we provide a way to classify and disentangle among the many different choices found in the literature for heavy fermions embeddings in $S O(5)$ [32].

Finally, we particularize the study to the fermionic scenario of Sect. 8.2. The heavy fermions there are integrated out explicitly together with the heavy scalar particle. Moreover, we do this both first integrating out the heavy fermions and then the heavy scalar and then the other way round; so it can be checked whether the order in which the computation is done has any effect on the results. 
By providing the benchmark Lagrangian for this kind of models, containing the first corrections from the linear renormalizable completion, we expect to provide a way to analyze the impact of a possible renormalizable UV completion and compare with the effects of scenarios with purely non-linear dynamics.

\subsection{Model independent analysis}

The considered Lagrangian has three parts:

$$
\mathcal{L}=\mathcal{L}_{s}+\mathcal{L}_{\mathrm{f}}+\mathcal{L}_{g}
$$

where $\mathcal{L}_{s}$ is the scalar Lagrangian of $(8.10)$, and $\mathcal{L}_{g}$ contains the SM kinetic terms for gauge bosons.

The fermionic part of the Lagrangian in Eq. (10.1) will be written as the sum of two terms:

$$
\mathcal{L}_{\mathrm{f}}=\mathcal{L}_{\mathrm{f}, \mathrm{SM}}^{\mathrm{kin}}+\mathcal{L}_{\mathrm{f}}^{\mathrm{Yuk}}
$$

where $\mathcal{L}_{\mathrm{f}, \mathrm{SM}}^{\text {kin }}$ comprises the kinetic terms for only SM fermions. As described in Sect. 8.2, a generic feature of models implementing partial compositeness is the addition of heavy vector-like fermions, which mix linearly with the SM fields, being in this way responsible for the explicit breaking of $S O(5)$, inducing a potential and mass for the Higgs particle at one-loop, and for the generation of SM fermion masses through effective Yukawa couplings.

The exact form of the effective coupling is model-dependent and varies according to how the SM fermions are embedded in $S O(5)$. However, at low energies it is possible to write an effective Yukawa Lagrangian in terms only of the SM fermions, plus $h$ and $\sigma$, which respects electroweak gauge invariance but breaks $S O(5)$,

$$
\mathcal{L}_{\mathrm{f}}^{\text {Yuk }} \equiv-y_{\mathrm{f}}^{0} \mathcal{O}_{\text {Yuk,f }}^{(n, m)}+\cdots+\text { h.c. },
$$

where the constant $y_{\mathrm{f}}^{0}$ is a model-dependent coefficient* and we define the effective Yukawa operator for a given fermion $\mathrm{f}$ (let us consider only quarks for simplicity) as

$$
\mathcal{O}_{\text {Yuk }, \mathrm{f}}^{(n, m)} \equiv \bar{q}_{L} \widetilde{H} \mathrm{f}_{R}\left(\frac{\sigma}{f}\right)^{n}\left(\frac{2 H^{\dagger} H}{f^{2}}\right)^{m},
$$

if $\mathrm{f}$ is an up-type quark, with $\widetilde{H} \equiv i \sigma^{2} H^{*}$ and

$$
\mathcal{O}_{\text {Yuk }, \mathrm{f}}^{(n, m)} \equiv \bar{q}_{L} H \mathrm{f}_{R}\left(\frac{\sigma}{f}\right)^{n}\left(\frac{2 H^{\dagger} H}{f^{2}}\right)^{m},
$$

if $\mathrm{f}$ is a down-type quark. The ellipses in Eq. (10.3) refer to other SM fermion operators and possibly extra model-dependent terms coming from the heavy fermion sector. The effective interaction between the SM fermions and the scalars is schematically shown in Fig. 10.1.

\footnotetext{
*The superscript 0 indicates that $y_{\mathrm{f}}^{0}$ encodes only the leading contributions induced by the heavy fermionic sector.
} 


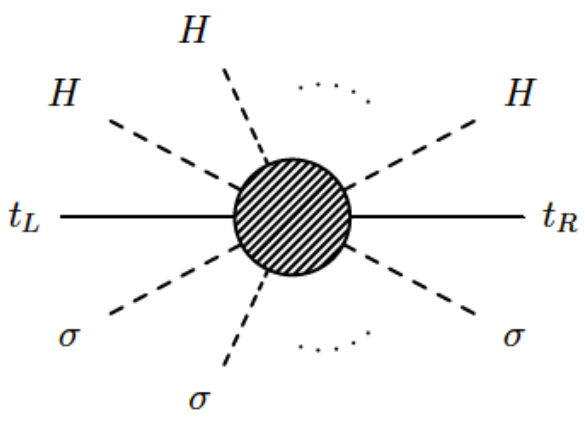

Figure 10.1: Schematic fermion mass operator at low scales with arbitrary insertions of the scalar fields.

\begin{tabular}{|c|c|}
\hline \hline Fermion representation $\left(q_{L^{-}} q_{R}\right)$ & Yukawa interactions $y_{\mathrm{f}}^{0} \mathcal{O}_{\text {Yuk }}^{(n, m)}$ \\
\hline \hline $5-1,5-10,10-5$ & $y \mathcal{O}_{\text {Yuk }}^{(0,0)}$ \\
\hline $5-5,10-10,14-10,10-14,14-1$ & $y \mathcal{O}_{\text {Yuk }}^{(1,0)}$ \\
\hline $14-14$ & $3 y \mathcal{O}_{\text {Yuk }}^{(1,0)}-2 y^{\prime} \mathcal{O}_{\text {Yuk }}^{(1,1)}+8 y^{\prime} \mathcal{O}_{\text {Yuk }}^{(3,0)}$ \\
\hline $14-5$ & $y \mathcal{O}_{\text {Yuk }}^{(0,0)}+y^{\prime} \mathcal{O}_{\text {Yuk }}^{(2,0)}$ \\
\hline $5-14$ & $y \mathcal{O}_{\text {Yuk }}^{(0,0)}+y^{\prime} \mathcal{O}_{\text {Yuk }}^{(0,1)}-4 y^{\prime} \mathcal{O}_{\text {Yuk }}^{(2,0)}$ \\
\hline \hline
\end{tabular}

Table 10.1: Yukawa operators corresponding to particular embeddings (see e.g. Ref [32]) of a SM quark doublet $q_{L}$ and right-handed $q_{R}$ fermion (either up-type or down-type right-handed quark) into $S O(5)$. The coefficients $y, y^{\prime}$ refer to distinct possible relative weights of $S O(5)$ invariant operators allowed by the models

In the literature of composite Higgs models the notation $\mathrm{MCHM}_{\mathrm{A}-\mathrm{B}-\mathrm{C}}$ is often used to indicate their fermion composition, with $\mathrm{A}, \mathrm{B}, \mathrm{C}$ indicating the $S O(5)$ representation in which the SM doublet $q_{L}$, up-type right-handed and down-type right-handed fermions are embedded, respectively. Also, when only one subindex appears as in $\mathrm{MCHM}_{\mathrm{A}}$ it is understood to be of the type $\mathrm{MCHM}_{\mathrm{A}-\mathrm{A}-\mathrm{A}}$. See Table 10.1 for a summary of the $\{n, m\}$ parameter values for different models ${ }^{\dagger}$.

Eqs. (10.4) and (10.5) assume that a given fermion mass corresponds to a single set of $\{n, m\}$ values. This is often the case; for instance, the top and bottom Yukawa couplings in the $\mathrm{MCHM}_{5-1-1}$ model of Sect. 8.2 correspond to $\mathcal{O}_{\text {Yuk }}^{(0,0)}$, while in the $\mathrm{MCHM}_{5}$ scenario they both correspond to $\mathcal{O}_{\text {Yuk }}^{(1,0)}$ (see e.g. Ref. [33]). Notice that, for these cases with a single Yukawa operator, the global coefficients and suppression scales in Eqs. (10.3)-(10.5) are constrained by the fermion masses and therefore do not constitute any additional model dependence.

\footnotetext{
${ }^{\dagger}$ Models with spinorial $S O(5)$ embeddings, e.g. $\mathrm{MCHM}_{4}$ [30], are phenomenologically excluded in particular in view of $Z \rightarrow b \bar{b}$ data [102]
} 
Nevertheless, in some scenarios a given fermion mass results instead from combining several operators of the type in Eqs. (10.4) and (10.5) with different $\{n, m\}$ values. The procedure derived can be easily extended to encompass it. A model-dependence remains then in the relative size of the $y$ and $y^{\prime}$ weights in Table 10.1. An example is the $\mathrm{MCHM}_{14-14-10}$ scenario [32] in which different sets of $\{n, m\}$ values are involved in generating the top mass, while the bottom mass only requires set $\{n, m\}=$ $\{1,0\}$. The cases of single and of multiple Yukawa operators contributing to a given mass will be further considered explicitly below. We focus in what follows on the top Yukawa coupling unless otherwise explicitly stated, while the conclusions to be obtained are easily generalized to all light fermions.

\section{Polar coordinates}

In order to derive the benchmark bosonic Lagrangian it is convenient to rewrite the scalar degrees of freedom in polar coordinates:

$$
\begin{aligned}
\sigma & =\rho c_{\varphi} \\
H & =\frac{1}{\sqrt{2}} \rho \mathbf{U} s_{\varphi},
\end{aligned}
$$

with $c_{\varphi} \equiv \cos \varphi / f, s_{\varphi} \equiv \sin \varphi / f$, and $\mathbf{U}(x)$ is the GB matrix. In this notation the scalar Lagrangian in Eq. (10.1) reads

$\mathcal{L}_{s}=\frac{1}{2} \partial_{\mu} \rho \partial^{\mu} \rho+\frac{\rho^{2}}{2 f^{2}}\left[\partial_{\mu} \varphi \partial^{\mu} \varphi-\frac{f^{2}}{2} s_{\varphi}^{2}\left\langle\mathbf{V}_{\mu} \mathbf{V}^{\mu}\right\rangle\right]-\lambda\left(\rho^{2}-f^{2}\right)^{2}-\alpha f^{3} \rho c_{\varphi}+\beta f^{2} \rho^{2} s_{\varphi}^{2}$,

where \langle\rangle denotes the trace and $\mathbf{V}_{\mu}=\left(D_{\mu} \mathbf{U}\right) \mathbf{U}^{\dagger}$ as in Eq. (5.12). The effective top Yukawa operator in Eqs. (10.3), (10.4) is then given by

$$
y_{t}^{0} \mathcal{O}_{\text {Yuk }, t}^{(n, m)}=\frac{y_{t}^{0}}{\sqrt{2}}\left(\bar{q}_{L} \mathbf{U} P_{+} \mathbf{q}_{R}\right) \rho\left(\frac{\rho}{f}\right)^{n+2 m} c_{\varphi}^{n} s_{\varphi}^{2 m+1}
$$

Note that the right-handed SM fermions have been gathered in a $S U(2)_{R}$ doublet, labeled by $\mathbf{q}_{R} \equiv\left(t_{R}, b_{R}\right)$ in order not to confuse with the heavy fermions $Q$ shown in Eq. (8.34). $P_{+}=\operatorname{diag}(1,0)$ and $P_{-}=\operatorname{diag}(0,1)$ are the projectors onto the up-type and down-type, respectively, right-handed SM fermions.

The $\rho$ and $\varphi$ fields will develop vevs,

$$
\rho \rightarrow \rho+\langle\rho\rangle, \quad \varphi \rightarrow h+\langle\varphi\rangle .
$$

At the minimum of the potential the $\varphi$ field corresponds to

$$
\cos \left(\frac{\langle\varphi\rangle}{f}\right)=-\frac{\alpha}{2 \beta}\left(1+\frac{\beta}{2 \lambda}\right)^{-1 / 2} .
$$

The connection between the vevs of the fields in the linear and polar parametrizations is

$$
\langle\rho\rangle=\sqrt{\langle\sigma\rangle^{2}+2\langle H\rangle^{2}}, \quad\langle\varphi\rangle=f \tan ^{-1}\left(\frac{\sqrt{2}\langle H\rangle}{\langle\sigma\rangle}\right) .
$$


Now the radial field $\rho$ will take the role of the heavy scale, previously taken by the $\sigma$ scalar. Indeed, $m_{\rho}=m_{\sigma}$, exactly, as expected for a physical observable; while the physical Higgs $h$ corresponds now to the excitation of the $\varphi$ field; see Eq. 10.10.

Finally, as the pure gauge Lagrangian $\mathcal{L}_{g}$ and the weak coupling to fermions are not modified, the coefficient of the $W_{\mu}$ mass term in Eq. (10.8) allows to identify the electroweak scale $v$ in terms of the Lagrangian parameters:

$$
v^{2}=\langle\rho\rangle^{2} \sin ^{2}\left(\frac{\langle\varphi\rangle}{f}\right)
$$

\section{Expansion in $1 / \lambda$}

The scalar quartic coupling $\lambda$ can be conventionally traded by the $\rho$ mass, given by $m_{\rho}^{2} \simeq 8 \lambda f^{2}$ for negligible $\alpha$ and $\beta$, see Eq. (8.19). The non-linear model would be recovered in the limit $m_{\rho} \gg f$; that is, $\lambda \rightarrow \infty$. Varying the $\rho$ mass (i. e., $\lambda$ ) allows to sweep from the regime of perturbative ultraviolet completion to the non-linear one assumed in models in which the Higgs particle is a low-energy remnant of some strong dynamics; which is the limit we want to explore in the following.

The exact equation of motion for $\rho$ reads

$$
\begin{aligned}
\square \rho-\frac{\rho}{f^{2}}[ & \left.\partial_{\mu} \varphi \partial^{\mu} \varphi-\frac{f^{2}}{2} s_{\varphi}^{2}\left\langle\mathbf{V}_{\mu} \mathbf{V}^{\mu}\right\rangle\right]+4 \lambda \rho\left(\rho^{2}-f^{2}\right)+\alpha f^{3} c_{\varphi}-2 \beta \rho f^{2} s_{\varphi}^{2} \\
& +(n+2 m+1)\left(\frac{y_{t}^{0}}{\sqrt{2}} \bar{q}_{L} \mathbf{U} P_{+} \mathbf{q}_{R}+\text { h.c. }\right) c_{\varphi}^{n} s_{\varphi}^{2 m+1}\left(\frac{\rho}{f}\right)^{n+2 m}=0
\end{aligned}
$$

where $\square \equiv \partial_{\mu} \partial^{\mu}$. In a $1 / \lambda$ expansion, the $\rho$ field can be expressed as

$$
\rho \equiv \rho_{0}+\rho_{1} / \lambda+\rho_{2} / \lambda^{2}+\ldots
$$

where the leading terms are given by

$$
\begin{aligned}
& \rho_{0}=f, \\
& \begin{aligned}
\rho_{1}=\frac{f}{4}\left[\frac{1}{2 f^{4}} \partial_{\mu} \varphi \partial^{\mu} \varphi\right. & -\frac{1}{4 f^{2}}\left\langle\mathbf{V}_{\mu} \mathbf{V}^{\mu}\right\rangle s_{\varphi}^{2}-\frac{1}{2} \alpha c_{\varphi}+\beta s_{\varphi}^{2} \\
& \left.\quad-\frac{(n+2 m+1)}{2 f^{3}}\left(\frac{y_{t}^{0}}{\sqrt{2}} \bar{q}_{L} \mathbf{U} P_{+} \mathbf{q}_{R}+\text { h.c. }\right) c_{\varphi}^{n} s_{\varphi}^{2 m+1}\right],
\end{aligned}
\end{aligned}
$$

and subsequent ones can be written as polynomial functions of $\rho_{1}$. Substituting these in Eq. (10.8) yields the $1 / \lambda^{n}$ Lagrangian corrections,

$$
\mathcal{L}=\mathcal{L}_{0}+\mathcal{L}_{1} / \lambda+\mathcal{L}_{2} / \lambda^{2}+\ldots
$$


where the different terms in this equation are given by

$$
\begin{aligned}
& \mathcal{L}_{0}=\frac{1}{2} \partial_{\mu} \varphi \partial^{\mu} \varphi-\frac{f^{2}}{4}\left\langle\mathbf{V}_{\mu} \mathbf{V}^{\mu}\right\rangle s_{\varphi}^{2}-\alpha f^{4} c_{\varphi}+\beta f^{4} s_{\varphi}^{2}-\frac{f y_{t}^{0}}{\sqrt{2}} \bar{q}_{L} \mathbf{U} P_{+} \mathbf{q}_{R} c_{\varphi}^{n} s_{\varphi}^{2 m+1}, \\
& \mathcal{L}_{1}=4 f^{2} \rho_{1}^{2}, \\
& \left.\mathcal{L}_{2}=\frac{1}{2}\left(\partial_{\mu} \rho_{1}\right)^{2}+\frac{\rho_{1}^{2}}{2}\left[\alpha f^{2} c_{\varphi}+\left(1-(n+2 m)^{2}\right)\left(\frac{f y_{t}^{0}}{\sqrt{2}} \bar{q}_{L} \mathbf{U} P_{+} \mathbf{q}_{R}+\text { h.c. }\right) c_{\varphi}^{n} s_{\varphi}^{2 m+1}\right)\right] .
\end{aligned}
$$

$\mathcal{L}_{0}$ coincides with the leading-order Lagrangian for the scalar sector of the minimal composite Higgs model [30], as expected. The expressions obtained for $\mathcal{L}_{1}$ and $\mathcal{L}_{2}$ are remarkably compact and a similar pattern holds for higher orders in $1 / \lambda$.

The maximum number of derivatives of $\mathcal{L}_{n}$ is $2+2 n$, although not all $2+2 n$ derivative operators are generated at order $n$. The ordering in which the operators appear is akin to the power counting of non-linear Higgs effective theory [100, 129, 130, $135]$.

Eqs. (10.16) and (10.19) suggest interesting correlations between operators involving the Higgs boson, gauge bosons and fermions. In particular, operators such as $\left(\partial_{\mu} h\right)^{2} \bar{\psi} \psi$ or $\left\langle\mathbf{V}_{\mu} \mathbf{V}^{\mu}\right\rangle \bar{\psi} \psi$, where $\psi$ denotes a generic fermion, are weighted by the fermion mass and also bear a dependence on the SM embedding into $S O(5)$, parametrized by the set $\{n, m\}$ in Eq. (10.9). From those equations emerges the low-energy effective Lagrangian in terms of SM fields at a given order in $1 / \lambda$,

$$
\mathcal{L}_{\text {eff }}=\mathcal{L}_{g}+\mathcal{L}_{\mathrm{f}, \mathrm{SM}}^{\text {kin }}+\sum_{i} \mathcal{P}_{i} \mathcal{F}_{i}(\varphi)
$$

where the first two terms in the right-hand side contain respectively the kinetic terms for gauge bosons and fermions as in Eqs. (10.1) and (10.2), and the index $i$ runs over all operator labels and coefficient functions $\mathcal{F}_{i}(\varphi)$ in Table 10.2. The table collects all couplings corresponding to two and four "derivatives", where plain derivatives and gauge boson insertions are counted with equal weight; since they come together in the covariant derivative.

We have found that, up to first order in the linear corrections, the benchmark effective Lagrangian is determined to be composed of ten operators, five of them bosonic and the rest fermionic ${ }^{\ddagger}$, including that responsible for Yukawa couplings. The coefficients of those operators are not free but intimately correlated by the coefficient functions explicitly determined in this work, and shown in the table.

Among the couplings which first appear at $\mathcal{O}(1 / \lambda)$, three bosonic operators are singled out in the $\mathrm{SO}(5)$-invariant limit $\left(\alpha=\beta=0\right.$, massless $\mathrm{SM}$ fermions): $\mathcal{P}_{6}$, $\mathcal{P}_{20}$ and $\mathcal{P}_{D H}$. The two latter ones involve multiple Higgs insertions and are out of present experimental reach; while the strength of $\mathcal{P}_{6}$, which involves vertices with four gauge bosons, is already tested directly by data, although the present sensitivity is very weak $[214,215]^{\S}$.

\footnotetext{
${ }^{\ddagger}$ For fermionic operators only the generic Lorentz and flavor structures are made explicit, being trivial their decomposition in terms of different flavors.

§These bounds can be translated for instance in $m_{\rho} \gtrsim 70 \mathrm{GeV}$ for $f \approx 600 \mathrm{GeV}$.
} 


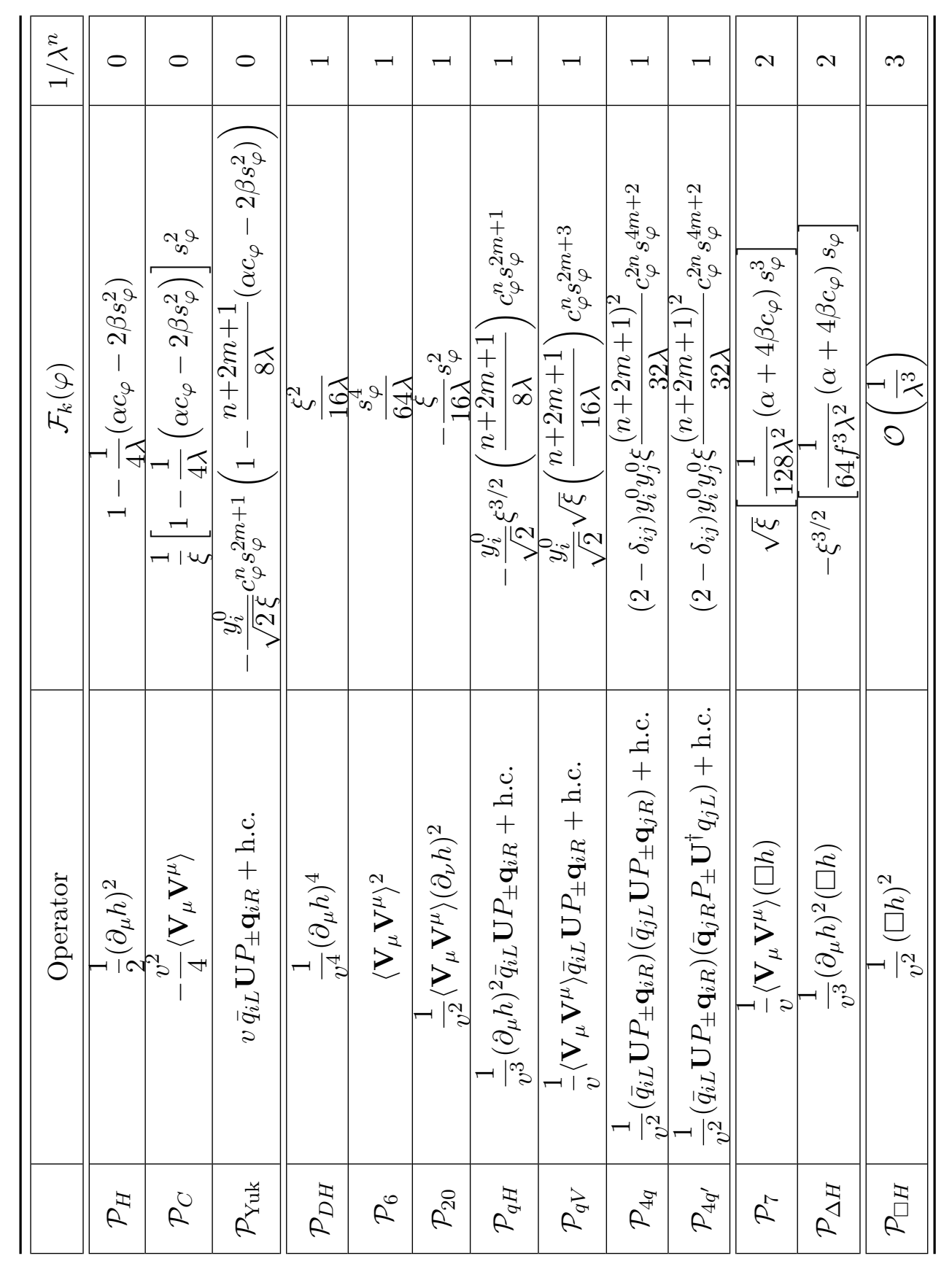

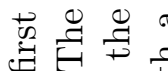

궁

Ð

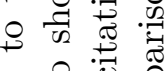

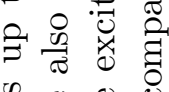

苞芯要

$\overrightarrow{0}^{\circ} \bigcirc$

模

슬

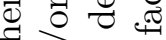

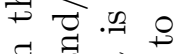

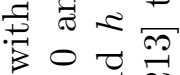

4 t

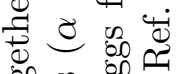

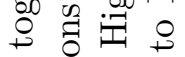

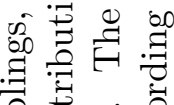

苛泀

0 bo

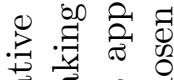

荡

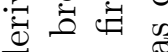

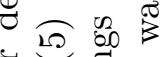

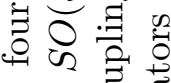

च छ

बै

ङ 50

$\infty 0$

:

吾昰青

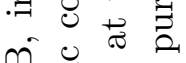

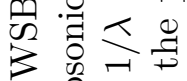

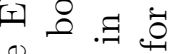

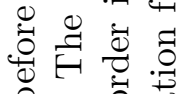

क.

每

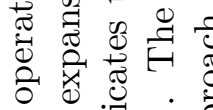

芒苛家 苑 里

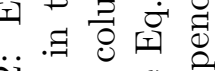
ช่

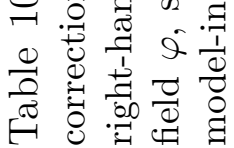


Operators involving SM fermions have an implicit dependence on the symmetrybreaking terms in the Lagrangian. Most interestingly, the corresponding $\mathcal{F}_{i}(\varphi)$ coefficients, written as a function of the $\{n, m\}$ parameters, allow to differentiate the expected impact of different fermionic ultraviolet completions in the literature.

The gauge field dependence is present only through powers of $\left\langle\mathbf{V}_{\mu} \mathbf{V}^{\mu}\right\rangle$, consistent with its exclusively scalar covariant derivative origin, see Eq. (10.8). Other chiralinvariant Lorentz contractions such as $\left\langle\mathbf{V}_{\mu} \mathbf{V}_{\nu}\right\rangle^{2}$ would be loop-induced, and thus expected to be subleading.

The scalar functions $\mathcal{F}_{i}(\varphi)$ obtained are in agreement with those derived in Ref. [213] in the $S O(5)$ invariant limit, for the subset of operators identified here as benchmarks; see their Eqs. (2.5)-(2.8). In addition, Table 10.2 also shows the leading deviations due to the presence of the explicit $S O(5)$-breaking parameters $\alpha$ and $\beta$.

\section{Impact on Higgs observables}

\section{Bosonic sector}

From Eqs. (10.18) and (10.19), the potential reads

$$
\frac{V}{f^{4}}=\alpha c_{\varphi}-\beta s_{\varphi}^{2}-\frac{1}{16 \lambda}\left(\alpha c_{\varphi}-2 \beta s_{\varphi}^{2}\right)^{2}+\mathcal{O}\left(\frac{1}{\lambda^{2}}\right)
$$

with minimum at $\cos \left(\frac{\langle\varphi\rangle}{f}\right) \simeq-\frac{\alpha}{2 \beta}\left(1-\frac{\beta}{4 \lambda}\right)$; see Eq.(10.11). The kinetic energy of the physical Higgs excitation $h$ (see Eq. (10.10)) gets then a correction given by

$$
\frac{1}{2}\left(1+\frac{\beta}{2 \lambda}\right) \partial_{\mu} h \partial^{\mu} h,
$$

which is reabsorbed by a field redefinition

$$
h \rightarrow\left(1+Z_{h}\right) h, \quad \text { with } \quad Z_{h}=-\frac{\beta}{4 \lambda} .
$$

The renormalization of the scalar sector can be made in terms of four observables as in Sect. 8.1, with $m_{\rho}=m_{\sigma}$ and the mixing is substituted by the modification to the Higgs coupling to the EW gauge bosons:

$$
\begin{gathered}
G_{F} \equiv\left(\sqrt{2} v^{2}\right)^{-1}, \quad m_{h}, \quad \kappa_{V}, \quad m_{\rho} \\
\Gamma\left(h \rightarrow W W^{*}\right) \equiv \Gamma_{\mathrm{SM}}\left(h \rightarrow W W^{*}\right) \kappa_{V}^{2} .
\end{gathered}
$$

The masses of the heavy and light resonances are given in (8.17), with the expressions in the limit of small $S O(5)$ explicit breaking, $|\beta| / 4 \lambda \ll 1$, given in (8.19). In the non-linear limit $\lambda \rightarrow \infty$ the $\rho$ field decouples from the spectrum and the scalar sector depends on just three renormalized parameters. It is now possible to foresee 
the impact of the linear corrections in terms of mass dependence. Precisely because large $\lambda$ implies large $m_{\rho}$, dimensional arguments suggest the equivalence

$$
\frac{1}{\lambda} \quad \Rightarrow \quad \frac{m_{h}^{2}}{m_{\rho}^{2}} \simeq \frac{\beta}{4 \lambda} \xi,
$$

as expansion parameter, see Eq. (8.19). In other words, the linear corrections are expected to be proportional to the two small parameters $\beta$ and $\xi$ and thus doubly suppressed.

A departure from 1 of the Higgs coupling to the vector bosons, given by $\kappa_{V}$ (see Eq. (10.26)), is generically expected. This is encoded, from Eqs. (10.17)-(10.20) at order $1 / \lambda$, in the operator $\mathcal{P}_{C}$ in table 10.2 and reads

$$
\mathcal{L}_{h V V}=-\left(\frac{1}{2} \sqrt{1-\xi}+\frac{\beta}{8 \lambda} \frac{(2-\xi) \xi}{\sqrt{1-\xi}}\right)\left\langle\mathbf{V}_{\mu} \mathbf{V}^{\mu}\right\rangle v h
$$

or in other words

$$
\kappa_{V}=\sqrt{1-\xi}+\frac{\beta \xi}{2 \lambda} \frac{(1-\xi / 2)}{\sqrt{1-\xi}} .
$$

Assuming for illustrative purposes $\mathcal{O}(\xi) \sim \mathcal{O}(1 / \lambda)$ and expanding up to second order in these parameters, the result simplifies to

$$
\kappa_{V} \simeq \sqrt{1-\xi}+\frac{\beta \xi}{2 \lambda}
$$

The first term on the right-hand side of this equation is the well-known correction present in non-linear scenarios [30], while the second term encodes the linear correction linked to the scale of ultraviolet completion, which in terms of physical parameters we predict to be given by

$$
\kappa_{V}^{2} \simeq 1-\xi+4 \frac{m_{h}^{2}}{m_{\rho}^{2}}
$$

where Eq. (10.27) has been used. Higher order corrections are expected to be very small, as they will originate from operators with at least 4 derivatives. For instance, the first extra tree-level contribution to $\kappa_{V}$ is order $1 / \lambda^{2}$, from the operator $\mathcal{P}_{7}$ in table 10.2:

$$
\delta \kappa_{V}^{2} \simeq \frac{1}{2 \sqrt{2} G_{F}} \frac{m_{h}^{2}}{m_{\rho}^{4}}
$$

\section{Fermionic sector}

Consider first the case in which the fermion mass is generated by a single Yukawa operator $\mathcal{O}_{\text {Yuk }, f}^{(n, m)}$ (see Eqs. (10.4) and (10.5)). From the Lagrangian in Eqs. (10.17)(10.20), and more specifically from the Yukawa operator in the third line of Table 10.2, an expression for the fermion mass follows after applying Eqs. (10.10) and (10.24):

$$
\begin{aligned}
\mathcal{L}_{\mathrm{f}}^{\text {Yuk }} & \supset-m_{\mathrm{f}} \overline{\mathrm{f}}_{L} \mathrm{f}_{R}+\text { h.c. } \\
m_{\mathrm{f}} & \simeq \frac{y_{\mathrm{f}}^{0}}{\sqrt{2}} f \sqrt{\xi}(1-\xi)^{n / 2} \xi^{m}\left(1+n \frac{1}{\xi(1-\xi)} \frac{m_{h}^{2}}{m_{\rho}^{2}}\right) .
\end{aligned}
$$


The renormalization scheme is now enlarged to the fermion sector choosing as observables precisely the fermion masses. The prediction that follows for the Higgs coupling to a given fermion $\mathrm{f}$,

$$
\mathcal{L}_{h \mathrm{ff}} \equiv-g_{h \mathrm{ff}} h \overline{\mathrm{f}}_{L} \mathrm{f}_{R}+\text { h.c. },
$$

takes then the form

$$
\begin{aligned}
g_{h \mathrm{ff}} \simeq \frac{y_{\mathrm{f}}^{0}}{\sqrt{2}} & (1-\xi)^{\frac{n-1}{2}} \xi^{m}\left\{(1+2 m)(1-\xi)-n \xi+\frac{\beta}{\xi(1-\xi)} \frac{m_{h}^{2}}{m_{\rho}^{2}} \times\right. \\
& \times[(1+2 m+n) \xi(1-\xi)(2-\xi)+n(1+2 m(1-\xi)-n \xi)]\} .
\end{aligned}
$$

Encoding the deviations with respect to the SM expectations through the conventional $\kappa_{\mathrm{f}}$ parameter,

$$
\kappa_{\mathrm{f}} \equiv g_{h \mathrm{ff}} / g_{h \mathrm{ff}}^{\mathrm{SM}}
$$

where $g_{h \mathrm{ff}}^{\mathrm{SM}}=m_{\mathrm{f}} / v$, we find the following somewhat lengthy expression for $\kappa_{\mathrm{f}}$ up to order $1 / \lambda$ :

$$
\kappa_{\mathrm{f}} \simeq \frac{(1+2 m)(1-\xi)-n \xi}{\sqrt{1-\xi}}+(2+4 m+3 n) \frac{m_{h}^{2}}{m_{\rho}^{2}},
$$

where once again Eq. (10.27) has been used. It is straightforward to check that the first term on the right-hand side of this equation reproduces well-known $\kappa_{\mathrm{f}}$ results for different models in the literature, which assume a non-linear realization. The second term gives instead the leading linear corrections. For instance, this equation leads to the following results for the $\mathrm{MCHM}_{5-1-1}$ (corresponding to $n=m=0$ in our parametrization) and $\mathrm{MCHM}_{5}$ (corresponding to $n=1, m=0$ ):

$$
\kappa_{\mathrm{f}}^{\mathrm{MCHM}_{5-1-1}} \simeq \sqrt{1-\xi}+2 \frac{m_{h}^{2}}{m_{\rho}^{2}}, \quad \kappa_{\mathrm{f}}^{\mathrm{MCHM}_{5}} \simeq \frac{1-2 \xi}{\sqrt{1-\xi}}+5 \frac{m_{h}^{2}}{m_{\rho}^{2}} .
$$

obtaining again at order $1 / \lambda$ a correction doubly suppressed as proportional to both $\beta$ and $\xi$ (see Eq. (10.27)).

The cases mentioned above correspond to situations in which only one effective Yukawa interaction arises. However, there are cases in which more than one Yukawa operators can appear; see Table 10.1. To illustrate what happens in this case, let us consider now a setup in which a given fermion mass corresponds to the combination of several $S O(5)$ invariant Yukawa operators:

$$
\mathcal{L}_{\mathrm{f}}^{\text {Yuk }}=-c_{(n, m)} \mathcal{O}_{\text {Yuk }, \mathrm{f}}^{(n, m)}+\cdots+\text { h.c. }
$$

where $c_{(n, m)}$ are related to the generators of $S O(5)$ and the fermion embedding in a given model. The procedure is still quite straightforward. The fermion mass will be a sum of contributions similar to that in Eq. (10.33), weighted by the coefficients $c_{(n, m)}$, and a similar combination protocol will apply to the obtention of the fermion-Higgs coupling $g_{h f f}$ and thus $\kappa_{\mathrm{f}}$. As an example, consider the $\mathrm{MCHM}_{14-14-10}$ scenario [32], in which the third family quark doublet and the right-handed top are embedded each in a 14-plet of $S O(5)$, denoted by $Q_{L}$ and $U_{R}$, respectively; while the right-handed 
bottom is included in a 10-plet representation denoted by $D_{R}$. Two $S O(5)$ invariant operators [32] contribute in this case to the top quark mass:

$$
y_{u} \phi^{\dagger} \bar{Q}_{L} U_{R} \phi-\tilde{y}_{u}\left(\phi^{\dagger} \bar{Q}_{L} \phi\right)\left(\phi^{\dagger} U_{R} \phi\right) \rightarrow 3 y_{u} \mathcal{O}_{\text {Yuk }}^{(1,0)}-\tilde{y}_{u}\left(2 \mathcal{O}_{\text {Yuk }}^{(1,1)}-8 \mathcal{O}_{\text {Yuk }}^{(3,0)}\right),
$$

leading to

$$
\kappa_{\mathrm{t}}^{\mathrm{MCHM}_{14-14-10}} \simeq \frac{y_{u}(3-6 \xi)+2 \tilde{y}_{u}\left(4-23 \xi+20 \xi^{2}\right)}{\sqrt{1-\xi}\left(3 y_{u}+2 \tilde{y}_{u}(4-5 \xi)\right)}+\frac{15 y_{u}^{2}+32 \tilde{y}_{u}\left(8 \tilde{y}_{u}-3 y_{u}\right)}{\left(8 \tilde{y}_{u}-3 y_{u}\right)^{2}} \frac{3 m_{h}^{2}}{m_{\rho}^{2}}
$$

On the other hand, in this example only one effective Yukawa operator contributes to the bottom quark mass,

$$
y_{d} \phi^{\dagger} \bar{Q}_{L} D_{R} \phi \rightarrow y_{d} \mathcal{O}_{\mathrm{Yuk}}^{(1,0)}
$$

and consequently

$$
\kappa_{\mathrm{b}}^{\mathrm{MCHM}_{14-14-10}} \simeq \frac{1-2 \xi}{\sqrt{1-\xi}}+\frac{5 \beta \xi}{4 \lambda} \simeq \frac{1-2 \xi}{\sqrt{1-\xi}}+\frac{5 m_{h}^{2}}{m_{\rho}^{2}} .
$$

Again, all $\mathcal{O}(1 / \lambda)$ corrections considered above show the double suppression in $\xi$ and $\beta$, which is equivalent to a $m_{h}^{2} / m_{\rho}^{2}$ suppression factor (Eq. (10.27)).

\subsection{Explicit fermion sector}

In the previous section, the heavy fermion sector was assumed to be integrated out from the start; while the corrections due to the heavy scalar singlets were explored. In this section we start instead with a complete (bosons plus fermions) renormalizable model, so as to estimate the impact of a fermionic ultraviolet completion beyond that related to the effective Yukawa couplings discussed above. The lowenergy effective Lagrangian made out of SM fields is then explicitly determined up to the leading corrections stemming from the heavy scalar and fermion sectors, up to $\mathcal{O}(1 / \lambda) \sim \mathcal{O}\left(m_{h}^{2} / m_{\rho}^{2}\right)$ and $\mathcal{O}\left(f / \mathcal{M}_{i}\right)$, respectively; where $\mathcal{M}_{i}$ denotes generically the heavy fermion masses.

We use now a fermionic Lagrangian

$$
\mathcal{L}_{\mathrm{f}}=\mathcal{L}_{\mathrm{f}}^{\text {kin }}+\mathcal{L}_{\mathrm{f}}^{\text {Yuk }},
$$

where $\mathcal{L}_{\mathfrak{f}}^{\text {kin }}$ contains now kinetic terms for all fermions, light and heavy, and the fermion mass Lagrangian denoted by $\mathcal{L}_{\mathrm{f}}^{\text {Yuk }}$ that needs to be specified for a particular ultraviolet fermion completion.

In particular, let us analyze the model described in Chapter 8 and 9. The fermion fields are shown in (8.34); while the complete fermionic Lagrangian can be seen in Eqs. (8.35) and (8.36). That particular choice of heavy fermion representations is equivalent to an $\mathrm{MCHM}_{5-1-1}$ scenario, and thus to the effective Yukawa operator $\mathcal{O}_{\text {Yuk,f }}^{(n, m)}$ in Eqs. (10.4) and (10.5) with $\{n, m\}=\{0,0\}$ (see entry $5-1$ in the first row of Table 10.1). 


\begin{tabular}{|c|c|}
\hline \hline Coefficient & Leading Order in $f / \mathcal{M}_{i}$ \\
\hline \hline $\mathcal{Z}_{q_{L}}$ & $\left(1+\frac{\Lambda_{1}^{2}}{M_{5}^{2}}+\frac{\Lambda_{1}^{\prime 2}}{M_{5}^{\prime 2}}\right)$ \\
\hline $\mathcal{Z}_{t_{R}}$ & $\left(1+\frac{\Lambda_{2}^{2}}{M_{5}^{2}}+\frac{\Lambda_{3}^{2}}{M_{1}^{2}}\right)$ \\
\hline $\mathcal{Z}_{b_{R}}$ & $\left(1+\frac{\Lambda_{2}^{\prime 2}}{M_{5}^{\prime 2}}+\frac{\Lambda_{3}^{\prime 2}}{M_{1}^{\prime 2}}\right)$ \\
\hline \hline
\end{tabular}

Table 10.3: Table with the definitions for the renormalization factors.

\section{Integrating out only the heavy fermions}

Let us integrate out only the heavy BSM fermions of the model, so we can provide here with a basis relevant for a regime in which $\mathcal{M}_{i} \gg m_{\sigma} \gg v$; where $\mathcal{M}_{i}$ is defined as a generic heavy fermion mass scale associated with the mass generation mechanism of a given SM fermion. For instance, for the top quark, $M_{1} \sim M_{5} \sim$ $\Lambda_{1} \sim \cdots \sim \mathcal{M}_{t}$ (see Eq. (8.35)). Assuming this scale to be larger than $f, f / \mathcal{M}_{i}$ is a good expansion parameter. In what follows we will thus assume $f / \mathcal{M}_{i} \ll 1$ for simplicity, and $\Lambda_{i} \approx \mathcal{M}_{i}$.

The procedure to integrate the heavy fermions out is actually quite lengthy; so only the resulting mass-dimension $(d) 4,5$ and 6 effective operators and their coefficients are summarized here. For energies $E<\mathcal{M}_{i}$, the effective Lagrangian describing the $d \leq 6$ interactions of fermions with gauge and scalar fields can be written as

$$
\mathcal{L}_{\text {eff }}=\bar{q}_{L} i \not D q_{L}+\bar{t}_{R} i \not D t_{R}+\bar{b}_{R} i \not D b_{R}+\sum_{i} c_{i} \mathcal{O}_{i}
$$

where the set $\left\{\mathcal{O}_{i}\right\}$ includes operators of dimension four (for which the induced coefficients are the leading contributions to the top and bottom Yukawa couplings), five and six.

The kinetic energies for the light fermion fields get contributions which require wave function renormalization in order to be canonically normalized:

$$
\begin{aligned}
q_{L} & \rightarrow \mathcal{Z}_{q_{L}}^{-1 / 2} q_{L} \\
q_{R} & \rightarrow\left(\begin{array}{cc}
\mathcal{Z}_{t_{R}}^{-1 / 2} & 0 \\
0 & \mathcal{Z}_{b_{R}}^{-1 / 2}
\end{array}\right)\left(\begin{array}{l}
t_{R} \\
b_{R}
\end{array}\right),
\end{aligned}
$$

where $\mathcal{Z}_{t_{R}}^{-1 / 2}$ and $\mathcal{Z}_{b_{R}}^{-1 / 2}$ are given in Table 10.3 .

The operators obtained and their coefficients resulting after those redefinitions, at leading order in $f / \mathcal{M}_{i}$, are shown in Table 10.48, where the following definitions have been used,

$$
\begin{aligned}
\left(H^{\dagger} i \overleftrightarrow{D}_{\mu} H\right) & \equiv i\left(H^{\dagger}\left(\vec{D}_{\mu} H\right)-\left(H^{\dagger} \overleftarrow{D}_{\mu}\right) H\right) \\
\left(H^{\dagger} i \overleftrightarrow{D}_{\mu}^{i} H\right) & \equiv i\left(H^{\dagger} \tau^{i}\left(\vec{D}_{\mu} H\right)-\left(H^{\dagger} \overleftarrow{D}_{\mu}\right) \tau^{i} H\right)
\end{aligned}
$$




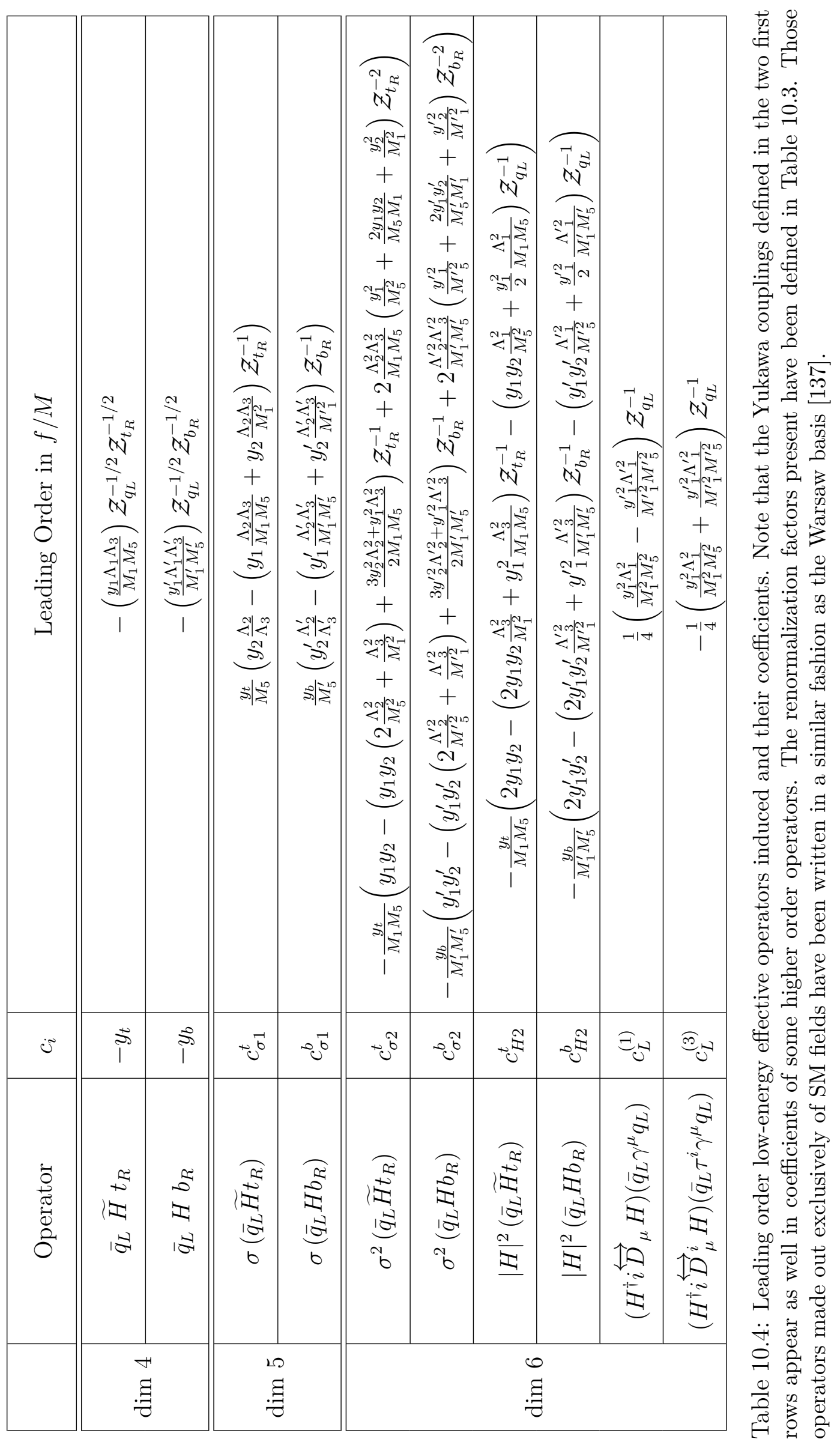


Interestingly, the coefficients for operators $\left(H^{\dagger} i \overleftrightarrow{D}_{\mu} H\right)\left(\bar{t}_{R} \gamma^{\mu} t_{R}\right),\left(H^{\dagger} i \overleftrightarrow{D}_{\mu} H\right)\left(\bar{b}_{R} \gamma^{\mu} b_{R}\right)$ and $i\left(\widetilde{H}^{\dagger} D_{\mu} H\right)\left(\bar{t}_{R} \gamma^{\mu} b_{R}\right)$ were found to vanish.

It is important to note that when writing Eq. (10.45) and Table 10.48 the scalar fields are emphnot shifted by their vevs; which introduces the following subtlety. Let us consider for instance the top and bottom quark masses corresponding to the first two operators in the table, which are their respective Yukawa couplings. When the Higgs field gets a vev, mass terms for them are generated. However, additional contributions to the light quark masses can originate from the next six operators in the list, for $\sigma=\langle\sigma\rangle$ and $H=\langle H\rangle$. The corrections induced in the top and bottom mass are of higher order in $f / \mathcal{M}_{i}$, though, and do not need to be retained when working at leading order. Finally, the expressions for the light fermion masses are:

$$
\begin{aligned}
& m_{t}=\frac{v}{\sqrt{2}}\left(\frac{y_{1} \Lambda_{1} \Lambda_{3}}{M_{1} M_{5}}\right) \frac{1}{\sqrt{\left(1+\frac{\Lambda_{1}^{2}}{M_{5}^{2}}+\frac{\Lambda_{1}^{\prime}}{M_{5}^{\prime 2}}\right)\left(1+\frac{\Lambda_{2}^{2}}{M_{5}^{2}}+\frac{\Lambda_{3}^{2}}{M_{1}^{2}}\right)}}\left(1+\mathcal{O}\left(\frac{f}{\mathcal{M}_{t}}\right)\right), \\
& m_{b}=\frac{v}{\sqrt{2}}\left(\frac{y_{1}^{\prime} \Lambda_{1}^{\prime} \Lambda_{3}^{\prime}}{M_{1}^{\prime} M_{5}^{\prime}}\right) \frac{1}{\sqrt{\left(1+\frac{\Lambda_{1}^{2}}{M_{5}^{2}}+\frac{\Lambda_{1}^{\prime}}{M_{5}^{\prime 2}}\right)\left(1+\frac{\Lambda_{2}^{\prime 2}}{M_{5}^{\prime 2}}+\frac{\Lambda_{3}^{\prime 2}}{M_{1}^{\prime 2}}\right)}}\left(1+\mathcal{O}\left(\frac{f}{\mathcal{M}_{b}}\right)\right) .
\end{aligned}
$$

The same reasoning applies to other couplings. For example the fermion- $\sigma$ coupling via the $\mathcal{O}_{\sigma 1}^{t}$ operator would get corrections proportional to $c_{\sigma 2}^{t}\langle\sigma\rangle$ (see Table 10.48), which are of higher order in the $f / \mathcal{M}_{i}$ expansion, and can thus be also disregarded when restraining to the leading contributions.

\section{Integrating out only the heavy scalar}

Above, we have integrated out only the heavy fermions, determining then the effective Lagrangian made out of SM fields plus the singlet scalar present in the minimal $S O(5)$ sigma model.

Let us now start by integrating out only the heavy scalar; so it is possible in the end to check whether he final low-energy effective Lagrangian made out only of SM fields is independent of the order in which the limits (heavy scalar and heavy BSM fermions) are taken.

Using polar coordinates and integrating out the radial mode $\rho$ does not bring any novel complication with respect to the procedure carried out in Sect. 10.1, except for lengthier expressions. $\mathcal{L}_{F}^{\text {Yuk }}$ can be compactly written prior to any integration procedure as

$$
\begin{aligned}
\mathcal{L}_{F}^{\text {Yuk }}=-[ & \rho\left(s_{\varphi} \mathcal{O}_{s}^{F}+c_{\varphi} \mathcal{O}_{c}^{F}\right)+\Lambda_{1} \bar{q}_{L} Q_{R}+\Lambda_{1}^{\prime} \bar{q}_{L} Q_{R}^{\prime} \\
& \left.+\Lambda_{2} \bar{T}_{L}^{(5)} t_{R}+\Lambda_{3} \bar{T}_{L}^{(1)} t_{R}+\Lambda_{2}^{\prime} \bar{B}_{L}^{(5)} b_{R}+\Lambda_{3}^{\prime} \bar{B}_{L}^{(1)} b_{R}+\text { h.c. }\right]
\end{aligned}
$$

where $\mathcal{O}_{s}^{F}$ and $\mathcal{O}_{c}^{F}$ are heavy fermion bilinears corresponding to the first four lines 
in Eq. (8.36):

$$
\begin{aligned}
\mathcal{O}_{s}^{F} & =-\frac{1}{\sqrt{2}}\left[y_{1}\left(\bar{X}_{L} \mathbf{U} e_{-} T_{R}^{(1)}+\bar{Q}_{L} \mathbf{U} e_{+} T_{R}^{(1)}\right)+y_{2}\left(\bar{T}_{L}^{(1)} \mathbf{U} e_{-} X_{R}+\bar{T}_{L}^{(1)} \mathbf{U} e_{+} Q_{R}\right)\right. \\
& \left.+y_{1}^{\prime}\left(\bar{X}_{L}^{\prime} \mathbf{U} e_{+} B_{R}^{(1)}+\bar{Q}_{L}^{\prime} \mathbf{U} e_{-} B_{R}^{(1)}\right)+y_{2}^{\prime}\left(\bar{B}_{L}^{(1)} \mathbf{U} e_{+} X_{R}^{\prime}+\bar{B}_{L}^{(1)} \mathbf{U} e_{-} Q_{R}^{\prime}\right)\right], \quad 10.4 \\
\mathcal{O}_{c}^{F} & =-\frac{1}{\sqrt{2}}\left[y_{1} \bar{T}_{L}^{(5)} T_{R}^{(1)}+y_{2} \bar{T}_{L}^{(1)} T_{R}^{(5)}+y_{1}^{\prime} \bar{B}_{L}^{(5)} B_{R}^{(1)}+y_{2}^{\prime} \bar{B}_{L}^{(1)} B_{R}^{(5)}\right]
\end{aligned}
$$

where $e_{+}=(1,0)$ and $e_{-}=(0,1)$.

Considering next the limit of very large scalar mass $m_{\rho}$ (i.e. $\lambda \rightarrow \infty$ ), the effective Lagrangian at $\mathcal{O}(1 / \lambda)$ takes again the form in Eq. (10.19), with $\rho_{1}$ showing now an explicit dependence on the heavy fermion spectrum:

$$
\begin{aligned}
\rho_{1}=\frac{f}{4}\left[\frac{1}{2 f^{4}} \partial_{\mu} \varphi \partial^{\mu} \varphi\right. & -\frac{1}{4 f^{2}}\left\langle\mathbf{V}_{\mu} \mathbf{V}^{\mu}\right\rangle s_{\varphi}^{2}-\frac{1}{2} \alpha c_{\varphi}+\beta s_{\varphi}^{2} \\
& \left.-\left\{\frac{1}{2 f^{3}} \mathcal{O}_{c}^{F} c_{\varphi}+\frac{1}{2 f^{3}} \mathcal{O}_{s}^{F} s_{\varphi}+\text { h.c. }\right\}\right],
\end{aligned}
$$

where the structures bearing the contributions from heavy BSM fermions are:

$$
\begin{aligned}
- & \frac{1}{8 \lambda f^{3}} \partial_{\mu} \varphi \partial^{\mu} \varphi\left(\mathcal{O}_{c}^{F} c_{\varphi}+\mathcal{O}_{s}^{F} s_{\varphi}\right) \\
& \frac{1}{16 \lambda f}\left\langle\mathbf{V}_{\mu} \mathbf{V}^{\mu}\right\rangle s_{\varphi}^{2}\left(\mathcal{O}_{c}^{F} c_{\varphi}+\mathcal{O}_{s}^{F} s_{\varphi}\right) \\
& \frac{1}{16 \lambda f^{2}}\left(\mathcal{O}_{c}^{F} c_{\varphi}+\mathcal{O}_{s}^{F} s_{\varphi}\right)^{2}
\end{aligned}
$$

\section{Combining the two limits}

In order to obtain the final effective Lagrangian, one can either integrate out the BSM fermions in (10.52), or integrate out the $\rho$ scalar in the operators of Table 10.48; after redefining the fields in terms of the polar ones. Since we are only interested in the dominant corrections from heavy fermions; the contributions from the dimension six operators in Table 10.48 will be disregarded.

Either way or another we obtain five effective operators up to first order in $1 / \lambda$ and $f / \mathcal{M}_{i}$; as is shown in Table 10.5. The $a_{\sigma 1}^{i}$ operator coefficients of the $f / \mathcal{M}_{i}$ corrections are expected to be $\mathcal{O}(1)$. They are a redefinition of the $c_{\sigma}^{i}$ operator coefficients in Table 10.48 so as to extract explicitly the $f / \mathcal{M}_{i}$ dependence:

$$
c_{\sigma 1}^{i} \rightarrow y_{t}^{0} a_{\sigma 1}^{t} / \mathcal{M}_{t} .
$$

The exact expressions for $c_{\sigma}^{i}$ for the fermion model discussed here can be found in Table 10.48.

Regarding the impact on observables, it is found that, at tree level, the heavy fermions have no effect on the gauge-Higgs coupling and $\kappa_{V}$ is still given by Eq. (10.29). 


\begin{tabular}{|c|c|c|}
\hline \hline & Operator & $\mathcal{F}_{i}(\varphi)$ \\
\hline $\mathcal{P}_{\text {Yuk }}$ & $v\left(\bar{q}_{i L} \mathbf{U} P_{ \pm} \mathbf{q}_{i R}\right)$ & $-\frac{y_{t}^{0}}{\sqrt{2 \xi}} s_{\varphi}\left[1-\frac{1}{8 \lambda}\left(\alpha c_{\varphi}-2 \beta s_{\varphi}^{2}\right)-2 \frac{f}{\mathcal{M}_{i}} a_{\sigma 1}^{i} c_{\varphi}\right]$ \\
\hline $\mathcal{P}_{q h}$ & $\left(\partial_{\mu} h\right)^{2}\left(\bar{q}_{i L} \mathbf{U} P_{ \pm} \mathbf{q}_{i R}\right)$ & $-\frac{y_{i}^{0}}{8 \sqrt{2} \lambda f^{3}} s_{\varphi}\left(1-2 \frac{f}{\mathcal{M}_{i}} a_{\sigma 1}^{i} c_{\varphi}\right)$ \\
\hline $\mathcal{P}_{q V}$ & $\left\langle\mathbf{V}_{\mu} \mathbf{V}^{\mu}\right\rangle\left(\bar{q}_{i L} \mathbf{U} P_{ \pm} \mathbf{q}_{i R}\right)$ & $\frac{y_{i}^{0}}{16 \sqrt{2} \lambda f} s_{\varphi}\left(1-2 \frac{f}{\mathcal{M}_{i}} a_{\sigma 1}^{i} c_{\varphi}\right)$ \\
\hline $\mathcal{P}_{4 q}$ & $\left(\bar{q}_{i L} \mathbf{U} P_{ \pm} \mathbf{q}_{i R}\right)\left(\bar{q}_{j L} \mathbf{U} P_{ \pm} \mathbf{q}_{j R}\right)$ & $\left(2-\delta_{i j}\right) \frac{y_{i}^{0} y_{j}^{0}}{32 \lambda f^{2}} s_{\varphi}^{2}\left[1-2\left(a_{\sigma 1}^{i} \frac{f}{\mathcal{M}_{i}}+a_{\sigma 1}^{j} \frac{f}{\mathcal{M}_{j}}\right) c_{\varphi}\right]$ \\
\hline $\mathcal{P}_{4 q^{\prime}}$ & $\left(\bar{q}_{i L} \mathbf{U} P_{ \pm} \mathbf{q}_{i R}\right)\left(\overline{\mathbf{q}}_{j R} P_{ \pm} \mathbf{U}^{\dagger} q_{j L}\right)$ & $\left(2-\delta_{i j}\right) \frac{y_{i}^{0} y_{j}^{0}}{32 \lambda f^{2}} s_{\varphi}^{2}\left[1-2\left(a_{\sigma 1}^{i} \frac{f}{\mathcal{M}_{i}}+a_{\sigma 1}^{j} \frac{f}{\mathcal{M}_{j}}\right) c_{\varphi}\right]$ \\
\hline \hline
\end{tabular}

Table 10.5: Effective operators, up to order $f / \mathcal{M}_{i}$ and $1 / \lambda$, after integrating out the radial mode $\rho$ and the heavy fermions in a UV realization of partial compositeness. The coefficients $a_{\sigma 1}^{f}$, with $f=t, b$ can be found in Table 10.48; see Eq. 10.53. The Hermitian conjugate should be included for all operators here. As done above, the Higgs field $h$ is defined as the excitation of the field $\varphi$ (see Eq. (10.10)).

The coupling to top quarks, on the contrary, receives fermionic contributions from the first operator in Table 10.5:

$$
\kappa_{\mathrm{t}}=\sqrt{1-\xi}+2 \frac{m_{h}^{2}}{m_{\rho}^{2}}+a_{\sigma 1}^{t} \frac{f}{\mathcal{M}_{t}} \xi+\ldots
$$

The leading heavy fermion corrections $\sim \xi f / \mathcal{M}_{t}$, are also found to be doubly suppressed, like the bosonic ones in $\sim \beta \xi /(2 \lambda)$. It is important to note, though, that the tree-level fermionic contributions found may be larger than those induced by the scalar sector if $f / \mathcal{M}_{t}>\beta / \lambda$. This may be of special importance for the top quark since the top partners should not be too heavy in order not to generate a new hierarchy problem.

In the limit $f / \mathcal{M}_{i} \rightarrow 0$, the operators in Table 10.5 coincide as expected with the fermion-Higgs and four fermion operators given previously in Table 10.2 using the effective Yukawa operator $\mathcal{O}_{\text {Yuk }}^{(0,0)}$. 


\section{Part IV}

\section{HEFT and Baryon Number Violation}





\section{Chapter 11}

\section{Baryon non-invariant couplings in Higgs effective field theory}

The reason why baryon number violating (BNV) processes are considered relevant is because they offer the possibility to tackle the matter-antimatter asymmetry in our universe [216-218]; which cannot be explained within the SM. Nevertheless, no BVN effects have been observed so far; despite the numerous experimental searches on decays of hadrons, heavy fermions, $Z$ boson and nucleons. The latter provides the most stringent constraints [41].

As an example of the impact of a non-linearly realized EWSB sector on new physics for baryon number violating processes, we present in this chapter the construction of the complete basis of operators violating at first-order baryon and lepton numbers, within the HEFT expansion [219].

The first attempts towards the description of an EFT for BNV processes were done in within the SMEFT framework, and go back to the late 1970's [220-223]; but is also contained in a few more recent studies $[136,137,224]$. At the lowest order in the expansion in the cut-off of the theory suppressing the operators, $1 / \Lambda_{B}$, four BNV independent non-renormalizable structures of canonical dimension $d=6$ with the SM particle content were identified

$$
\begin{aligned}
\mathcal{O}_{1} & =\bar{d}_{R \alpha}^{C} u_{R \beta} \bar{Q}_{L \gamma i}^{C} L_{L j} \epsilon_{i j} \epsilon_{\alpha \beta \gamma}, \\
\mathcal{O}_{2} & =\bar{Q}_{L i \alpha}^{C} Q_{L j \beta} \bar{u}_{R \gamma}^{C} e_{R} \epsilon_{i j} \epsilon_{\alpha \beta \gamma}, \\
\mathcal{O}_{3} & =\bar{Q}_{L i \alpha}^{C} Q_{L j \beta} \bar{Q}_{L \gamma k}^{C} L_{L l} \epsilon_{i l} \epsilon_{k j} \epsilon_{\alpha \beta \gamma}, \\
\mathcal{O}_{4} & =\bar{d}_{R \alpha}^{C} u_{R \beta} \bar{u}_{R \gamma}^{C} e_{R} \epsilon_{\alpha \beta \gamma},
\end{aligned}
$$

where here we chose the notation such that $Q_{L} \equiv\left(u_{L}, d_{L}\right)^{T}, L_{L} \equiv\left(\nu_{L}, e_{L}\right)^{T}, u_{R}$, $d_{R}$ and $e_{R}$ are the SM fermions; $\epsilon_{\alpha \beta \gamma}$ and $\epsilon_{i j}$ are the antisymmetric tensors for the color and weak isospin contractions, respectively. Only one generation of fermions is considered. Moving to the three generation case does not require the introduction of additional structures, but only to insert explicitly flavor indices on the fermion 
fields. In addition, if $\mathrm{RH}$ neutrinos, $N_{R}$, are also considered, the set is extended by two operators:

$$
\begin{aligned}
& \mathcal{O}_{5}=\bar{Q}_{L i \alpha}^{C} Q_{L j \beta} \bar{d}_{R \gamma}^{C} N_{R} \epsilon_{i j} \epsilon_{\alpha \beta \gamma}, \\
& \mathcal{O}_{6}=\bar{u}_{R \alpha}^{C} d_{R \beta} \bar{d}_{R \gamma}^{C} N_{R} \epsilon_{\alpha \beta \gamma} .
\end{aligned}
$$

The operators in Eqs. (11.1) and (11.2) preserve $B-L$ with $\Delta B=+1=\Delta L$; so they can be related to baryon decay processes into an anti-lepton and a meson. The constraints on the proton lifetime [225-227] translate into a lower bound on the cut-off $\Lambda_{B}$ of about $10^{15} \mathrm{GeV}$, independently of the specific flavor contraction that can be considered for each operator. On the contrary, when a flavor symmetry is considered, such as the so-called Minimal Flavor Violation (MFV) ansatz in its global [228-236] or gauged [237-243] versions, the scale $\Lambda_{B}$ can be lowered, but still it will be much larger than the EW scale.

\subsection{The BNV HEFT Lagrangian}

One could expect that the basis of BNV operators introduced in Eqs. (11.1) and (11.2) will not be modified in the HEFT framework, as they are purely fermionic. Indeed, these six operators appear again simply rewritten in terms of $S U(2)_{L}$ and $S U(2)_{R}$ fermion doublets. However, the fact that the GB matrix $\mathbf{U}$ and $\mathbf{T}$ are adimensional allows to construct additional independent structures also with canonical dimension $d=6$, bearing interactions that appear at higher orders in the SMEFT; as it will be discussed below.

In short, the set of BNV operators within the HEFT formalism, at the first order in the expansion on $\Lambda_{B}$, consists of 12 independent structures:

$$
\begin{aligned}
\mathcal{R}_{1} & =\bar{Q}_{L i \alpha}^{C} Q_{L j \beta} \bar{Q}_{L k \gamma}^{C} L_{L l} \epsilon_{i l} \epsilon_{k j} \epsilon_{\alpha \beta \gamma} \mathcal{F}_{1}(h), \\
\mathcal{R}_{2} & =\bar{Q}_{L i \alpha}^{C} Q_{L j \beta} \bar{Q}_{L k \gamma}^{C}\left(\mathbf{T} L_{L}\right)_{l} \epsilon_{i l} \epsilon_{k j} \epsilon_{\alpha \beta \gamma} \mathcal{F}_{2}(h), \\
\mathcal{R}_{3} & =\bar{Q}_{R i \alpha}^{C} Q_{R j \beta} \bar{Q}_{R k \gamma}^{C} L_{R l} \epsilon_{i l} \epsilon_{k j} \epsilon_{\alpha \beta \gamma} \mathcal{F}_{3}(h), \\
\mathcal{R}_{4} & =\bar{Q}_{R i \alpha}^{C} Q_{R j \beta} \bar{Q}_{R k \gamma}^{C}\left(\mathbf{U}^{\dagger} \mathbf{T} \mathbf{U} L_{R}\right)_{l} \epsilon_{i l} \epsilon_{k j} \epsilon_{\alpha \beta \gamma} \mathcal{F}_{4}(h), \\
\mathcal{R}_{5} & =\bar{Q}_{R i \alpha}^{C} Q_{R j \beta} \bar{Q}_{L k \gamma}^{C} L_{L l} \epsilon_{i j} \epsilon_{k l} \epsilon_{\alpha \beta \gamma} \mathcal{F}_{5}(h), \\
\mathcal{R}_{6} & =\bar{Q}_{R i \alpha}^{C} Q_{R j \beta} \bar{Q}_{L k \gamma}^{C}\left(\mathbf{T} L_{L}\right)_{l} \epsilon_{i j} \epsilon_{k l} \epsilon_{\alpha \beta \gamma} \mathcal{F}_{6}(h), \\
\mathcal{R}_{7} & =\left(\bar{Q}_{R \alpha}^{C} \mathbf{U}^{t}\right)_{i}\left(\mathbf{T} \mathbf{U} Q_{R \beta}\right)_{j} \bar{Q}_{L k \gamma}^{C} L_{L l} \epsilon_{i l} \epsilon_{k j} \epsilon_{\alpha \beta \gamma} \mathcal{F}_{7}(h), \\
\mathcal{R}_{8} & =\left(\bar{Q}_{R \alpha}^{C} \mathbf{U}^{t}\right)_{i}\left(\mathbf{T} \mathbf{U} Q_{R \beta}\right)_{j} \bar{Q}_{L k \gamma}^{C}\left(\mathbf{T} L_{L}\right)_{l} \epsilon_{i l} \epsilon_{k j} \epsilon_{\alpha \beta \gamma} \mathcal{F}_{8}(h), \\
\mathcal{R}_{9} & =\bar{Q}_{L i \alpha}^{C} Q_{L j \beta} \bar{Q}_{R k \gamma}^{C} L_{R l} \epsilon_{i j} \epsilon_{k l} \epsilon_{\alpha \beta \gamma} \mathcal{F}_{9}(h), \\
\mathcal{R}_{10} & =\bar{Q}_{L i \alpha}^{C} Q_{L j \beta} \bar{Q}_{R k \gamma}^{C}\left(\mathbf{U}^{\dagger} \mathbf{T} \mathbf{U} L_{R}\right)_{l} \epsilon_{i j} \epsilon_{k l} \epsilon_{\alpha \beta \gamma} \mathcal{F}_{10}(h), \\
\mathcal{R}_{11} & =\left(\bar{Q}_{L \alpha}^{C} \mathbf{U}^{*}\right)_{i}\left(\mathbf{U}^{\dagger} \mathbf{T} Q_{L \beta}\right)_{j} \bar{Q}_{R k \gamma}^{C} L_{R l} \epsilon_{i l} \epsilon_{k j} \epsilon_{\alpha \beta \gamma} \mathcal{F}_{11}(h), \\
\mathcal{R}_{12} & =\left(\bar{Q}_{L \alpha}^{C} \mathbf{U}^{*}\right)_{i}\left(\mathbf{U}^{\dagger} \mathbf{T} Q_{L \beta}\right)_{j} \bar{Q}_{R k \gamma}^{C}\left(\mathbf{U}^{\dagger} \mathbf{T} \mathbf{U} L_{R}\right)_{l} \epsilon_{i l} \epsilon_{k j} \epsilon_{\alpha \beta \gamma} \mathcal{F}_{12}(h) .
\end{aligned}
$$

All the operators in this list have canonical mass dimension $d=6$ and therefore are suppressed by $\Lambda_{B}^{2}$. When ignoring $\mathrm{RH}$ neutrinos, the number of independent 
operators reduces to 9 . In particular, $\mathcal{R}_{4}, \mathcal{R}_{10}$ and $\mathcal{R}_{12}$ turn out to be vanishing or redundant with respect to the other structures. Among these 12 operators, 4 structures preserve custodial symmetry: $\mathcal{R}_{1}, \mathcal{R}_{3}, \mathcal{R}_{5}$ and $\mathcal{R}_{9}$. Indeed, they do not contain the custodial spurion $\mathbf{T}$.

The basis has structures that can be classified into four distinct classes: schematically, $Q_{L} Q_{L} Q_{L} L_{L}(L L L L), Q_{R} Q_{R} Q_{R} L_{R}(R R R R), Q_{L} Q_{L} Q_{R} L_{R} \quad(L L R R)$ and $Q_{R} Q_{R} Q_{L} L_{L}(R R L L)$. Notice also that all the operators can be written in terms of Lorentz scalar currents, being the other type of contractions vanishing or redundant via Fierz identities. Finally, it is worth stressing all the operators preserve $B-L$ (see next section for a detailed discussion on this).

\subsection{Comparison with the SMEFT}

In this section we compare the list of operators obtained within the HEFT framework with the BNV SMEFT operators in Eqs. (11.1) and (11.2). This serves as an example of various of the differences between linear and non-linear bases in Sect. 5.4.

First of all, the HEFT basis contains a larger number of structures (twelve) than the SMEFT one (only six). In both cases the operators have canonical dimension 6 and are thus suppressed by $1 / \Lambda_{B}^{2}$. As it has been pointed out a couple of times already, this is an important characteristic of the HEFT formalism; due to the adimensionality of $\mathbf{T}$ and $\mathbf{U}$. The HEFT operators which do not contain GBs can be straightforwardly related to the SMEFT set of $d=6$ structures:

$$
\begin{aligned}
\mathcal{R}_{1} & \rightarrow \mathcal{O}_{3}, \\
\mathcal{R}_{3} & \rightarrow \mathcal{O}_{4}+\mathcal{O}_{6}, \\
\mathcal{R}_{4} & \rightarrow-\mathcal{O}_{4}+\mathcal{O}_{6}, \\
\mathcal{R}_{5} & \rightarrow-\mathcal{O}_{1}, \\
\mathcal{R}_{9} & \rightarrow \mathcal{O}_{2}-\mathcal{O}_{5}, \\
\mathcal{R}_{10} & \rightarrow-\mathcal{O}_{2}-\mathcal{O}_{5} .
\end{aligned}
$$

Among these, operators $\mathcal{R}_{4}$ and $\mathcal{R}_{10}$ contain the combination $\mathbf{U}^{\dagger} \mathbf{T} \mathbf{U}$; but this actually simplifies to $\tau_{3}$ once using the definition of $\mathbf{T}$ in Eq. (5.13). This list shows that there is a linear correspondence between 6 operators of the HEFT basis and the 6 operators of the $d=6$ SMEFT one. The other HEFT operators contain interactions that can be described by SMEFT operators with dimension 8. An example is the following:

$$
\mathcal{R}_{2} \rightarrow \bar{Q}_{L i \alpha}^{C} Q_{L j \beta} \bar{Q}_{L k \gamma}^{C}\left[\left(\tilde{\Phi} \tilde{\Phi}^{\dagger}-\Phi \Phi^{\dagger}\right) L_{L}\right]_{l} \epsilon_{i l} \epsilon_{k j} \epsilon_{\alpha \beta \gamma}
$$

where the $h$-independent couplings of the combination $\tilde{\Phi} \tilde{\Phi}^{\dagger}-\Phi \Phi^{\dagger}$ in the unitary gauge play the same role as the scalar chiral field $\mathbf{T}$ in $\mathcal{R}_{2}$. Finally, notice also that only two combinations of SMEFT operators, $\mathcal{O}_{4}-\mathcal{O}_{6}$ and $\mathcal{O}_{2}+\mathcal{O}_{5}$, contain sources of custodial symmetry breaking; while most operators in Eq. (11.3) are custodial symmetry breaking (all are custodial violating except for $\mathcal{R}_{1}, \mathcal{R}_{3}, \mathcal{R}_{5}$ and $\mathcal{R}_{9}$ ).

Secondly, it is important to note that all the operators in both basis preserve $B-L$. In the HEFT formalism this is a consequence of hypercharge invariance. The reason 
for this is that hypercharge can be identified with $B-L$ in theories invariant under the $S U(2)_{L} \times S U(2)_{R}$ symmetry, such as in left-right symmetric models $[244,245]$. In these frameworks, because the $\mathrm{RH}$ fermions also belong to an $S U(2)$ doublet representation, and they have the same electric charge as their LH counterparts, both LH and RH fields must have the same hypercharge, -1 for leptons and 1/3 for quarks, in a given convention. This can be easily seen from the decomposition of the local transformation under $U(1)_{Y}$ of the LH and RH fermions; which, in a compact notation, ca be written in terms of $(B-L)$ as:

$$
\begin{aligned}
\psi_{L} & \rightarrow e^{i(B-L) \theta(x)} \psi_{L} \\
\psi_{R} & \rightarrow e^{i(B-L) \theta(x)} e^{i \theta(x) \tau_{3}} \psi_{R},
\end{aligned}
$$

where $\theta(x)$ is the hypercharge transformation parameter. Furthermore, the only source of custodial symmetry breaking we have in this formalism is $\mathbf{T}$; which does preserve the third component of $S U(2)_{R}$. Therefore, from Eq. (11.6), since it does not carry hypercharge, it means it cannot violate $(B-L)$. As a consequence, since hypercharge must be conserved, the only way to write $(B-L)$ non-conserving operators will be to introduce new structures, violating transformations under the third component of $S U(2)_{R}$; which are not found in conventional HEFT; as it will be shown in the example just below.

By contrast, in the SMEFT Lagrangian; where hypercharge and $B-L$ are independent, it is possible to find $B-L$ non-invariant operators; but it can be proved these are only found at odd-dimensions; following directly from Lorentz and hypercharge invariance [246]. The lowest dimensional example is the lepton number violating dimension five effective operator $\left(\bar{L}_{L}^{c} \tilde{\Phi}^{*}\right)\left(\tilde{\Phi}^{\dagger} L_{L}\right)$; known as the Weinberg operator [247]. As explained in the previous paragraph, different sources of $S U(2)_{R}$ violation other than $\mathbf{T}$ need be considered. For example, it is possible to write the analog of the Weinberg operator by taking the Pauli matrix $\sigma_{+}=\left(\sigma_{1}+i \sigma_{2}\right) / 2$; see [248] and also small discussion in [249]:

$$
\left(\bar{L}_{L}^{c} \mathbf{U}^{*}\right) \sigma_{+}\left(\mathbf{U}^{\dagger} L_{L}\right) \text {. }
$$

This operator preserves hypercharge, but violates $S U(2)_{R}$ and lepton number by two units, as it can be seen by writing explicitly the transformation under hypercharge of the GB matrix:

$$
\mathbf{U}(x) \rightarrow \mathbf{U}(x) e^{-i \theta(x) \tau_{3}} .
$$

Notice that this is a three dimensional operator and therefore provides a direct mass term for the light active neutrinos. In contrast, the Weinberg operator in the SMEFT is of $d=5$ and thus suppressed by a power of the mass scale at which lepton number is broken. Again, this is another example of the strong impact of the adimensionality of the GB matrix $\mathbf{U}$ with respect to the $S U(2)_{L}$ doublet Higgs of the SMEFT. In any case, in the rest of this chapter, no other sources of $S U(2)_{R}$ violation will be considered beside $\mathbf{T}$, consistently with previous studies in the HEFT context.

In conclusion, as expected, there is not a one-to-one relation between the HEFT and SMEFT sets of operators. Several correlations typical of the SMEFT are lost 
in the HEFT and that some couplings that are expected to be strongly suppressed in the SMEFT are instead predicted to be relevant in HEFT. As an example, in the comparison between the decay rates of the proton and of the neutron: $\Gamma\left(p \rightarrow \pi^{0} e^{+}\right)$ and $\Gamma\left(n \rightarrow \pi^{0} \bar{\nu}_{e}\right)$, in the $d=6$ SMEFT framework, the values of these two observables are predicted to be exactly the same; while this correlation can be broken considering $d=8$ operators. However, in the HEFT context, the operators $R_{2}, R_{6}$, $R_{7}, R_{8}, R_{11}, R_{12}$ contribute differently to the two decay rates, so no correlation arises at any order, in general. An experimental discrepancy among these two observables could then be explained either in terms of the SMEFT, but advocating $d=8$ contributions, or in terms of the HEFT Lagrangian. The magnitude of the discrepancy is what could tell which is the correct description: a relative difference between the two decay rates larger than about $\left(v^{2} / \Lambda_{B}^{2}\right)^{2}$ cannot be compatible with the $d=8$ SMEFT Lagrangian, and instead could well be accounted for in the HEFT context. At present, the non-observation of the proton decay puts a lower bound on the ratio $\Lambda_{B} / c_{i}$ of about $10^{15} \mathrm{GeV}$, where $c_{i}$ represents the combination of the operator coefficients entering the proton decay rate. As a result, this strategy to disentangle the two frameworks is an interesting feature from the theoretical side, although experimentally is not viable yet. Moreover, it allows to estimate the order of magnitude of the contributions to these decay rates from the $d=8$ SMEFT operators of about $10^{-51}$, with respect to those from the $d=6$ ones.

\subsection{Flavor contraction counting}

So far we have made no reference to the flavor structure of the operators. The number of independent flavor contractions can be counted directly considering the symmetries of the operators in Eq. (11.3). Alternatively, one can adopt the Hilbert series technique. This is a mathematical method from Invariant Theory to count the number of independent structures invariant under a certain symmetry group (for recent phenomenology applications see Refs. [250-255]). Basically, this technique provides a polynomial function whose terms can be matched with the operators in Eq. (11.3) and the corresponding coefficients count the number of independent flavor contractions. Although the matching is straightforward in the absence of scalar fields, as for the BNV HEFT operators considered here, one should be careful when dealing with structures containing the fields $\mathbf{T}$ and $\mathbf{U}$, being necessary to remove the redundancies due to $\mathbf{T}^{2}=\mathbb{1}$ and $\mathbf{U}^{\dagger} \mathbf{U}=\mathbb{1}$ a posteriori.

Let us consider $N_{f}$ fermion families and go through the four cases described in Sect. 11.1; namely $L L L L, R R R R, R R L L$ and $L L R R$, one by one:

- LLLL: The counting for $\mathcal{R}_{1}$ is $N_{f}^{2}\left(2 N_{f}^{2}+1\right) / 3$ and coincides with the one given in Ref. [224], where it is discussed in terms of flavor representations by using Young tableaux. The counting of $\mathcal{R}_{2}$ is the same as $\mathcal{R}_{1}$, as $\mathbf{T}$ only adds a flip of sign in the second component of the lepton doublet. When applying the Hilbert series technique, it seems there are more structures contributing to these type; but as it is warned above, a few cases with $\mathbf{T}$ insertions had to be subtracted from the total counting by hand for being redundant. 
- RRRR: $\mathcal{R}_{3}$ and $\mathcal{R}_{4}$ counting obviously simply mirrors that of the $L L L L$ case, so each operator presents $N_{f}^{2}\left(2 N_{f}^{2}+1\right) / 3$ flavor contractions. This does not seem consistent with the results in the SMEFT case (see Refs. [224, 256]), where the total number of flavor contractions for the $R R R R$ structures, $\mathcal{O}_{4}$ and $\mathcal{O}_{6}$, is $2 N_{f}^{4}$. This apparent contradiction is easily solved noticing that the $S U(2)_{R}$ symmetry is still partially preserved in the operators $\mathcal{R}_{3}$ and $\mathcal{R}_{4}$ and prevents part of the possible flavor contractions among four RH singlet fermions. Indeed, rewriting explicitly the flavor indices $a, b, c, d$, one gets

$$
\begin{aligned}
& \mathcal{R}_{3}^{a b c d}=\mathcal{O}_{4}^{\{b c\} a d}+\mathcal{O}_{6}^{\{b c\} a d}, \\
& \mathcal{R}_{4}^{a b c d}=-\mathcal{O}_{4}^{\{b c\} a d}+\mathcal{O}_{6}^{\{b c\} a d},
\end{aligned}
$$

where the brackets should be read as $\mathcal{O}_{i}^{\{a b\} c d} \equiv \mathcal{O}_{i}^{a b c d}+\mathcal{O}_{i}^{b a c d}$. This shows that $\mathcal{R}_{3}$ and $\mathcal{R}_{4}$ only contain the flavor symmetric contractions in $b$ and $c$ of the SMEFT operators. The flavor antisymmetric contractions should instead be described by two additional structures:

$$
\begin{aligned}
& \mathcal{R}_{3}^{\prime}=\bar{Q}_{R i \alpha}^{C}\left(\mathbf{U}^{\dagger} \mathbf{T} \mathbf{U} Q_{R \beta}\right)_{j} \bar{Q}_{R k \gamma}^{C} L_{R l} \epsilon_{i l} \epsilon_{k j} \epsilon_{\alpha \beta \gamma}, \\
& \mathcal{R}_{4}^{\prime}=\bar{Q}_{R i \alpha}^{C}\left(\mathbf{U}^{\dagger} \mathbf{T} \mathbf{U} Q_{R \beta}\right)_{j} \bar{Q}_{R k \gamma}^{C}\left(\mathbf{U}^{\dagger} \mathbf{T} \mathbf{U} L_{R}\right)_{l} \epsilon_{i l} \epsilon_{k j} \epsilon_{\alpha \beta \gamma} .
\end{aligned}
$$

These two operators are redundant with respect to $R_{3}$ and $R_{4}$ for $N_{f}=1$; but they should be added to the list in Eq. (11.3) for $N_{f}>1$ (see Ref. [223] for a similar discussion in the SMEFT). The number of the flavor contractions of these four $R R R R$ operators sums up to $2 N_{f}^{4}$; matching the result in the SMEFT case.

- RRLL: among these operators, only $\mathcal{R}_{5}$ can be directly related to a $d=6$ operator of the SMEFT Lagrangian (see Eq. (11.4)). However, rewriting the expression for $\mathcal{R}_{5}$, making explicit the flavor indices, one can see that actually $\mathcal{R}_{5}$ only contains part of the interactions described by $\mathcal{O}_{1}$ :

$$
\mathcal{R}_{5}^{a b c d}=-\mathcal{O}_{1}^{\{a b\} c d} .
$$

Similarly, the operator $\mathcal{R}_{6}^{a b c d}$ also contains only the flavor contractions symmetric in $a$ and $b$. It is therefore necessary to introduce two additional operators that completely break the $S U(2)_{R}$ structure between the first two $S U(2)_{R}$ quark doublets in $\mathcal{R}_{5}$ and $\mathcal{R}_{6}$ :

$$
\begin{aligned}
& \mathcal{R}_{5}^{\prime}=\bar{Q}_{R i \alpha}^{C}\left(\mathbf{U}^{\dagger} \mathbf{T} \mathbf{U} Q_{R \beta}\right)_{j} \bar{Q}_{L k \gamma}^{C} L_{L l} \epsilon_{i j} \epsilon_{k l} \epsilon_{\alpha \beta \gamma} \mathcal{F}_{5}(h) . \\
& \mathcal{R}_{6}^{\prime}=\bar{Q}_{R i \alpha}^{C}\left(\mathbf{U}^{\dagger} \mathbf{T} \mathbf{U} Q_{R \beta}\right)_{j} \bar{Q}_{L k \gamma}^{C}\left(\mathbf{T} L_{L}\right)_{l} \epsilon_{i j} \epsilon_{k l} \epsilon_{\alpha \beta \gamma} \mathcal{F}_{6}(h) .
\end{aligned}
$$

Analogously to the previous case, these two structures are redundant with $\mathcal{R}_{5}$ and $\mathcal{R}_{6}$ for $N_{f}=1$; otherwise they should be added to the basis; since $\mathcal{R}_{5}^{\prime}$ and $\mathcal{R}_{6}^{\prime}$ contain the interactions with the combinations antisymmetric in $a$ and $b$. In this way, $\mathcal{R}_{5}$ and $\mathcal{R}_{5}^{\prime}$ provide altogether all the flavor contractions of the SMEFT operator $\mathcal{O}_{1}$. On the other hand, the interactions of $\mathcal{R}^{6}$ and $\mathcal{R}_{6}^{\prime}$ are described by a $d=8$ operator of the SMEFT Lagrangian. Finally, the 
independent structures contained in the two remaining $R R L L$ operators, $\mathcal{R}_{7}$ and $\mathcal{R}_{8}$, read in the unitary gauge

$$
\bar{u}_{R \alpha a}^{C} u_{R \beta b} \bar{d}_{L \gamma c}^{C} e_{L d} \varepsilon_{\alpha \beta \gamma}, \quad \bar{d}_{R \alpha a}^{C} d_{R \beta b} \bar{u}_{L \gamma c}^{C} \nu_{L d} \varepsilon_{\alpha \beta \gamma},
$$

and are non-vanishing only for the combinations antisymmetric in $a$ and $b$. As a result, the number of independent flavor contractions for each of these two operators is $N_{f}^{3}\left(N_{f}-1\right) / 2$.

- LLRR: the interactions in $\mathcal{R}^{9}$ and $\mathcal{R}^{10}$ are described by linear combinations of the operators $\mathcal{O}_{2}$ and $\mathcal{O}_{5}$ of the SMEFT Lagrangian; as in Eq. (11.4), and the number of flavor contractions is $N_{f}^{3}\left(N_{f}+1\right) / 2$ for each of them; in agreement with Refs. [224,256]. On the other hand, the counting of the flavor contractions in $\mathcal{R}_{11}$ and $\mathcal{R}_{12}$ is analogous to the one for their $R R L L$ counterparts, $\mathcal{R}_{7}$ and $\mathcal{R}_{8}$; that is, $N_{f}^{3}\left(N_{f}-1\right) / 2$.

As a summary of the previous discussion, the number of flavor contractions can be summarized for the different operators as follows:

$$
\begin{array}{lll}
\mathcal{R}_{1} \rightarrow N_{f}^{2}\left(2 N_{f}^{2}+1\right) / 3 & \mathcal{R}_{7} & \rightarrow N_{f}^{3}\left(N_{f}-1\right) / 2 \\
\mathcal{R}_{2} \rightarrow N_{f}^{2}\left(2 N_{f}^{2}+1\right) / 3 & \mathcal{R}_{8} & \rightarrow N_{f}^{3}\left(N_{f}-1\right) / 2 \\
\mathcal{R}_{3}, \mathcal{R}_{3}^{\prime} \rightarrow N_{f}^{4} & \mathcal{R}_{9} & \rightarrow N_{f}^{3}\left(N_{f}+1\right) / 2 \\
\mathcal{R}_{4}, \mathcal{R}_{4}^{\prime} \rightarrow N_{f}^{4} & \mathcal{R}_{10} \rightarrow N_{f}^{3}\left(N_{f}+1\right) / 2 \\
\mathcal{R}_{5}, \mathcal{R}_{5}^{\prime} \rightarrow N_{f}^{4} & \mathcal{R}_{11} \rightarrow N_{f}^{3}\left(N_{f}-1\right) / 2 \\
\mathcal{R}_{6}, \mathcal{R}_{6}^{\prime} \rightarrow N_{f}^{4} & \mathcal{R}_{12} \rightarrow N_{f}^{3}\left(N_{f}-1\right) / 2 .
\end{array}
$$

In brief, this analysis completes previous studies on the HEFT Lagrangian by providing within that framework the complete set of operators at first-order that are not invariant under baryon and lepton numbers (but do preserve the $B-L$ combination). A detailed comparison with the SMEFT Lagrangian is also presented, pointing out a strategy to distinguish between the two approaches. Finally, the number of independent flavor contractions is also discussed. 


\section{Summary and conclusions}

The focus of this thesis was set on the study of the possibility that the Higgs particle descends from a Goldstone boson, originated in the spontaneous breaking of an approximate symmetry that would help protect its mass against quantum corrections.

Among the original results of this work, we explored the one-loop off-shell renormalization of the scalar sector of the effective non-linear Lagrangian with a light Higgs $h$. The full set of four-derivative custodial-invariant scalar operators required by the off-shell renormalization of all the 1-, 2-, 3- and 4- point functions involving GBs and the $h$ particle was determined. The results confirmed that the generic low-energy effective chiral Lagrangian with a light Higgs previously developed is complete in the scalar sector, as all the operators stated there (and nothing else) have been found to be required by the renormalization procedure. All computations were performed in a general parametrization of the GB matrix, showing that all counterterms induced by the renormalization procedure are independent of the parametrization chosen, as physical couplings should be. For completeness, the RGEs for the coefficients of the obtained counterterms have been computed as well. This could be useful in the future to compare experimental data at different energies.

Interestingly, performing an off-shell renormalization has also allowed us to identify chiral non-invariant divergences (NIDs) arising due to the perturbative procedure. These are shown to have no physical effect, as expected. They extend the set of NIDs previously found in the non-linear $\sigma$ model [146] to a set including also the Higgs particle. We showed that there exists a local field redefinition of the GBs that allows to absorb all apparently chiral non-invariances, thus proving that chiral symmetry actually remains unbroken.

As a further step, the renormalization of the HEFT Lagrangian has been performed. Instead of computing all the possible one-loop diagrams (as it was done in the first part of this work, regarding the scalar sector of the Lagrangian), we have used the powerful covariant derivative expansion. Moreover, the Lagrangian is formulated in terms of a geometric description of scalars in HEFT. As a result, we determine the complete set of necessary counterterms and the one-loop RGEs for the non-linear basis.

On a different approach, we developed and analyzed a particular renormalizable model based on a $S O(5) \rightarrow S O(4)$ spontaneous breaking in order to gain insight on the possible dynamics behind non-linear realizations of EWSB with a light Higgs 
particle. After including the minimal set of counterterms required by the ColemanWeinberg effective potential at one-loop, characterized by two arbitrary coefficients, we give a detailed analysis of the parameter space of the scalar potential in terms of the mass of the extra scalar, $\sigma$, and its mixing with the Higgs particle. In this way, we have identified the most interesting region for phenomenology, where the Higgs is a pGB and the hierarchy problem is alleviated. However, while the GB nature of the Higgs would protect its mass from large corrections, the $\sigma$ scalar mass would be unprotected from becoming heavy.

We provide a constraint on the mixing between the $h$ and $\sigma$ scalars from current LHC Higgs data: $\sin ^{2} \gamma<0.18$ at $2 \sigma$. The contributions of scalars and exotic fermions to precision parameters $\Delta S$ and $\Delta T$ and the modification of the $Z \bar{b} b$ coupling were also computed. We find that a not too heavy $\sigma$ particle is somewhat preferred, easing the possible tension on $\mathrm{S}$ and $\mathrm{T}$ from heavy fermions, usually present in the literature. The translation of the LHC bounds from heavy scalars searches onto the $\sigma$ parameter space of our model was also performed. Focusing on the region in which the Higgs can be considered as having a pGB origin, we found that run-1 LHC data already set a lower bound on the $\sigma$ mass of around $m_{\sigma}>550 \mathrm{GeV}$.

Finally, the connection with effective field theory is recovered: the heavy scalar mass allows to sweep from the linear sigma model to the non-linear regime in the heavy $\sigma$ mass limit. In this way we determined the benchmark effective Lagrangian from the $S O(5) \rightarrow S O(4)$ linear sigma model and also the first linear corrections. The latter allow to estimate the effect of a linear completion in non-linear implementations.

The effective benchmark operators found are shown to constitute a reduced set of the most general basis of bosonic operators for $S O(5) / S O(4)$ theories with no explicit breaking. In addition, we provide the dominant contributions stemming from the explicit $S O(5)$ breaking in our model, including the impact from heavy fermions. This impact is quite model-dependent, unlike the results for the scalar sector. For this reason, a model-independent approach based on an effective Yukawa interaction for the light fermions was developed in order to handle the dominant fermionic effects for different fermion setups. The effective Lagrangian was also derived using the explicit fermion embedding chosen in our renormalizable model, showing that the resulting operators match those provided by the corresponding effective Yukawa operator. Furthermore, the impact on Higgs couplings to EW gauge bosons and SM fermions, resulting from integrating out the heavy sector, is computed and found to be rather universal, with the linear corrections appearing to be doubly suppressed: both by the EW versus GB scales ratio $\left(v^{2} / f^{2}\right)$ and the parameters controlling the explicit versus spontaneous breakings $(\beta / \lambda)$.

As an additional exploration of the possible impact of a non-linear EWSB implementation, we have constructed, within the HEFT formalism, the basis of baryon number non-invariant operators, relevant to explain the matter-antimatter asymmetry of the visible Universe. A detailed comparison with the corresponding operators in the SMEFT basis is also provided. As usual, the number of operators in HEFT is found to be larger than in SMEFT at the same order in the expansion. A discussion on the number of independent flavor contractions is presented as well, considering 
the Hilbert series method to count invariants.

In conclusion, we have provided solid foundation for the choice of NLO effective operators for a given LO theory in the context of non-linear EWSB, at least from the point of view of renormalization. Furthermore, the idea of a Higgs particle with a pGB nature has been studied in a simple renormalizable implementation, which may be considered as a UV completion by itself, and is also used as a handle to clarify the interplay between the linear and non-linear regimes. 


\section{Resumen y conclusiones}

El objetivo de esta tesis ha sido el estudio de la posibilidad de que la partícula Higgs descienda de un bosón de Goldstone, originado en la ruptura espontánea de una simetría aproximada, la cual ayuda a proteger su masa de correcciones cuánticas.

Entre los resultados originales de este trabajo, está el estudio de la renormalización fuera de la capa de masas a 1 loop del sector escalar del Lagrangiano efectivo no-lineal para un Higgs ligero $h$. El conjunto completo de operadores escalares invariantes bajo simetría custodia de cuatro derivadas requeridos por la renormalización fuera de la capa de masas de todas las funciones de correlación de 1, 2, 3 y 4 puntos que involucran BGs y el Higgs $h$ fue determinado. Los resultados confirman que el Lagrangiano quiral efectivo de baja energía genérico con un Higgs ligero desarrollado en trabajos previos está completo en el sector escalar, dado que todos los operadores en la base de dichos trabajos (y nada más) son requeridos por el procedimiento de renormalización. Todos los cálculos fueron realizados en una parametrización general de la matriz de BGs, mostrando que todos los contra-términos inducidos por el procedimiento de renormalización son independientes de la parametrización elegida, tal como deberían ser los acoplos físicos. Por completitud, las EGRs correspondientes a los coeficientes de los contra-términos obtenidos han sido computados también. Esto podría resultar útil en el futuro para comparar datos experimentales a diferentes energías.

Curiosamente, realizar una renormalización fuera de la capa de masas también nos ha permitido identificar divergencias no-invariantes quirales, las cuales aparecen como consecuencia de aplicar un procedimiento perturbativo. Se muestra que no tienen consecuencias físicas, como es de esperar. Extienden el conjunto de divergencias noinvariantes encontradas previamente en el modelo $\sigma$ no-lineal [146] a un conjunto que incluye también la partícula de Higgs. Se muestra que existe una redefinición local de los campos de los BGs que permite absorber todas las no-invariancias quirales, y por tanto se demuestra que la simetría quiral se mantiene, de hecho, intacta.

Como continuación, se ha realizado la renormalización del Lagrangiano efectivo nolineal con un Higgs ligero completo. En lugar de calcular todos los diagramas posibles a 1 loop (como se hizo en la primera parte de este trabajo para la renormalización del sector escalar del Lagrangiano), hemos utilizado técnicas funcionales basadas en una expansión en las derivadas covariantes. Además, se utiliza una descripción geométrica de los escalares para formular el Lagrangiano efectivo. Como resultado, hemos determinado el conjunto completo de contra-términos necesarios y las EGRs 
a 1 loop para la base no-lineal.

De manera complementaria, hemos desarrollado y analizado un modelo renormalizable particular basado en una ruptura espontánea $S O(5) \rightarrow S O(4)$ para entender mejor la dinámica subyacente a las realizaciones no-lineales de la RESE con una partícula de Higgs ligera. Tras incluir el conjunto mínimo de contra-términos requeridos por el potencial efectivo de Coleman-Weinberg a 1 loop, caracterizados por dos coeficientes arbitrarios, mostramos un análisis detallado del espacio de parámetros del potencial escalar en términos de la masa del escalar extra, $\sigma$, y su mezcla con el Higgs. De esta manera, hemos identificado la región más interesante para la fenomenología, donde el Higgs es un pBG y el problema de la jerarquía es aliviado. Sin embargo, aunque la naturaleza GB del Higgs podría proteger su masa de grandes correcciones, el escalar $\sigma$ estaría desprotegido frente a volverse pesado.

Encontramos una cota para el ángulo de mezcla entre los escalares $h$ y $\sigma$ a partir de los datos actuales para el Higgs del LHC: $\sin ^{2} \gamma<0.18$ a $2 \sigma$. Las contribuciones de escalares y fermiones exóticos a los parámetros de precisión $\Delta S$ y $\Delta T$ y la modificación del acoplo $Z \bar{b} b$ también han sido computadas. Se encuentra que una partícula $\sigma$ no demasiado pesada es preferida, aliviando la posible tensión en $\mathrm{S}$ y $\mathrm{T}$ de los fermiones pesados, típicamente presente en la literatura. La traducción de los límites del LHC de búsquedas de escalares pesados sobre el espacio de parámetros del $\sigma$ de nuestro modelo también ha sido realizada. Centrándonos en la región en la que el Higgs puede ser considerado como pBG en origen, encontramos que los datos obtenidos en el run-1 del LHC ya establecen un límite inferior en la masa del sigma en torno a $m_{\sigma}>550 \mathrm{GeV}$.

Finalmente, recuperamos la conexión con la teoría efectiva de campos desde el modelo. La masa del sigma permite hacer un barrido del modelo sigma lineal al régimen no-lineal en el límite de masa muy pesada. De esta manera determinados el Lagrangiano efectivo de referencia desde el modelo sigma lineal $S O(5) \rightarrow S O(4)$ junto con las primeras correcciones lineales. Esto último permite estimar el efecto en implementaciones no-lineales de un modelo lineal que las complete en el ultravioleta.

Los operadores efectivos encontrados constituyen un conjunto reducido de la base más general de los operadores bosónicos para teorías $S O(5) / S O(4)$ sin ruptura explícita de la simetría. Adicionalmente, proveemos las contribuciones dominantes que vienen de la ruptura $S O(5)$ explícita en nuestro modelo, incluyendo el impacto de los fermiones pesados. Este impacto es bastante dependiente del modelo, no así los resultados del sector escalar. Por esta razón, un enfoque independiente del modelo basado en una interacción de Yukawa efectiva es desarrollada para así estudiar los efectos dominantes para diferentes configuraciones de fermiones en la literatura. El Lagrangiano efectivo fue también derivado utilizando fermiones en las representaciones particulares elegidas en nuestro modelo renormalizable, mostrando que los operadores resultantes coinciden con aquellos calculados a partir del operator Yukawa efectivo correspondiente. Además, ha sido calculado el impacto en los acoplos del Higgs a bosones gauge ED y fermiones del ME, resultantes de integrar el sector pesado. Este resultó ser bastante universal, con las correcciones lineales que aparecen suprimidas doblemente: tanto por el ratio de escalas ED vs. BG $\left(v^{2} / f^{2}\right)$ como 
por el ratio entre los parámetros que controlan la ruptura explícita vs. espontánea $(\beta / \lambda)$.

Como una exploración adicional del posible impacto de una implementación RSED no-lineal, hemos construido, dentro del formalismo de la TEC quiral para un Higgs ligero, la base de operadores que no conservan el número bariónico, relevantes para explicar la asimetría materia-antimateria del Universo visible. También se ha realizado una comparación detallada de los correspondientes operadores en la base TEC del ME Como es habitual, el número de operadores en la TEC quiral para un Higgs ligero resultó ser mayor que en la TEC del ME al mismo orden. Se ha presentado también una discusión acerca del número de contracciones de sabor independientes, considerando el método de las series de Hilbert para contar invariantes.

En conclusión, hemos proporcionado una fundamentación sólida para la elección de operadores efectivos a segundo orden para una teoría a primer orden dada en el contexto de una RSED no-lineal, al menos desde el punto de vista de la renormalización. Además, la idea de una partícula Higgs con un origen como pBG ha sido estudiada en una implementación renormalizable simple, que podría ser considerada como una terminación en el ultravioleta por sí misma, y es también utilizada como herramienta para clarificar la interacción entre los regímenes lineal y no-lineal. 


\section{Acknowledgements}

I am grateful to my supervisor, Belén, and to Pedro, Luca and Rodrigo for their patience. Also to my other collaborators Kirill, Ferruccio and Stefano.

I also wish to thank my family for supporting me throughout this time. To Miguel, for enduring the last part and in general to all my friends and to everybody who kept me company and/or helped me these years. 


\section{Bibliography}

[1] CMS Collaboration Collaboration, S. Chatrchyan et. al., Observation of a new boson at a mass of $125 \mathrm{GeV}$ with the CMS experiment at the $\mathrm{LHC}$, Phys.Lett. B716 (2012) 30-61, [arXiv:1207.7235].

[2] ATLAS Collaboration Collaboration, G. Aad et. al., Observation of a new particle in the search for the Standard Model Higgs boson with the ATLAS detector at the LHC, Phys.Lett. B716 (2012) 1-29, [arXiv:1207.7214].

[3] G. S. Guralnik, C. R. Hagen, and T. W. B. Kibble, Global Conservation Laws and Massless Particles, Phys. Rev. Lett. 13 (1964) 585-587.

[4] F. Englert and R. Brout, Broken Symmetry and the Mass of Gauge Vector Mesons, Phys.Rev.Lett. 13 (1964) 321-323.

[5] P. W. Higgs, Broken symmetries, massless particles and gauge fields, Phys.Lett. 12 (1964) 132-133.

[6] P. W. Higgs, Broken Symmetries and the Masses of Gauge Bosons, Phys.Rev.Lett. 13 (1964) 508-509.

[7] H. Georgi and D. B. Kaplan, Composite Higgs and Custodial SU(2), Phys.Lett. B145 (1984) 216.

[8] G. Passarino, NLO Inspired Effective Lagrangians for Higgs Physics, Nucl.Phys. B868 (2013) 416-458, [arXiv:1209.5538].

[9] C. Grojean, E. E. Jenkins, A. V. Manohar, and M. Trott, Renormalization Group Scaling of Higgs Operators and $\Gamma(h \rightarrow \gamma \gamma)$, JHEP 1304 (2013) 016, [arXiv: 1301.2588].

[10] J. Elias-Miro, J. Espinosa, E. Masso, and A. Pomarol, Renormalization of dimension-six operators relevant for the Higgs decays $h \rightarrow \gamma \gamma, \gamma Z$, JHEP 1308 (2013) 033, [arXiv:1302.5661].

[11] J. Elias-Miro, J. Espinosa, E. Masso, and A. Pomarol, Higgs windows to new physics through $d=6$ operators: constraints and one-loop anomalous dimensions, JHEP 1311 (2013) 066, [arXiv: 1308.1879].

[12] R. Contino, M. Ghezzi, C. Grojean, M. Muhlleitner, and M. Spira, Effective Lagrangian for a light Higgs-like scalar, JHEP 1307 (2013) 035, [arXiv:1303.3876]. 
[13] E. E. Jenkins, A. V. Manohar, and M. Trott, Renormalization Group Evolution of the Standard Model Dimension Six Operators I: Formalism and lambda Dependence, JHEP 1310 (2013) 087, [arXiv:1308.2627].

[14] E. E. Jenkins, A. V. Manohar, and M. Trott, Renormalization Group Evolution of the Standard Model Dimension Six Operators II: Yukawa Dependence, JHEP 01 (2014) 035, [arXiv:1310.4838].

[15] R. Alonso, E. E. Jenkins, A. V. Manohar, and M. Trott, Renormalization Group Evolution of the Standard Model Dimension Six Operators III: Gauge Coupling Dependence and Phenomenology, JHEP 04 (2014) 159, [arXiv:1312.2014].

[16] A. C. Longhitano, Low-Energy Impact of a Heavy Higgs Boson Sector, Nucl.Phys. B188 (1981) 118.

[17] A. C. Longhitano, Heavy Higgs Bosons in the Weinberg-Salam Model, Phys.Rev. D22 (1980) 1166.

[18] T. Appelquist and C. W. Bernard, Strongly Interacting Higgs Bosons, Phys.Rev. D22 (1980) 200.

[19] F. Feruglio, The Chiral approach to the electroweak interactions, Int.J.Mod.Phys. A8 (1993) 4937-4972, [hep-ph/9301281].

[20] B. Grinstein and M. Trott, A Higgs-Higgs bound state due to new physics at a TeV, Phys.Rev. D76 (2007) 073002, [arXiv:0704.1505].

[21] R. Contino, C. Grojean, M. Moretti, F. Piccinini, and R. Rattazzi, Strong Double Higgs Production at the LHC, JHEP 1005 (2010) 089, [arXiv:1002.1011].

[22] J. F. Donoghue, C. Ramirez, and G. Valencia, $\pi-\pi$ Scattering and Chiral Lagrangians, Phys. Rev. D38 (1988) 2195.

[23] M. J. Herrero and E. Ruiz Morales, The Electroweak chiral Lagrangian for the Standard Model with a heavy Higgs, Nucl. Phys. B418 (1994) 431-455, [hep-ph/9308276].

[24] M. J. Herrero and E. Ruiz Morales, Nondecoupling effects of the SM higgs boson to one loop, Nucl. Phys. B437 (1995) 319-355, [hep-ph/9411207].

[25] R. L. Delgado, A. Dobado, and F. J. Llanes-Estrada, One-loop $W_{L} W_{L}$ and $Z_{L} Z_{L}$ scattering from the electroweak Chiral Lagrangian with a light Higgs-like scalar, JHEP 1402 (2014) 121, [arXiv:1311.5993].

[26] D. Espriu, F. Mescia, and B. Yencho, Radiative corrections to $W_{L} W_{L}$ scattering in composite Higgs models, Phys.Rev. D88 (2013) 055002, [arXiv:1307.2400].

[27] R. Delgado, A. Dobado, M. Herrero, and J. Sanz-Cillero, One-loop $\gamma \gamma \rightarrow W_{L}^{+} W_{L}^{-}$and $\gamma \gamma \rightarrow Z_{L} Z_{L}$ from the Electroweak Chiral Lagrangian with a light Higgs-like scalar, arXiv:1404.2866. 
[28] F.-K. Guo, P. Ruiz-Femenia, and J. J. Sanz-Cillero, One loop renormalization of the electroweak chiral Lagrangian with a light Higgs boson, Phys. Rev. D92 (2015) 074005, [arXiv:1506.04204].

[29] M. Gell-Mann and M. Levy, The axial vector current in beta decay, Nuovo Cim. 16 (1960) 705.

[30] K. Agashe, R. Contino, and A. Pomarol, The Minimal composite Higgs model, Nucl.Phys. B719 (2005) 165-187, [hep-ph/0412089].

[31] R. Contino, D. Marzocca, D. Pappadopulo, and R. Rattazzi, On the effect of resonances in composite Higgs phenomenology, JHEP 1110 (2011) 081, [arXiv:1109.1570].

[32] M. Carena, L. Da Rold, and E. Pontón, Minimal Composite Higgs Models at the LHC, JHEP 06 (2014) 159, [arXiv:1402.2987].

[33] G. Panico and A. Wulzer, The Composite Nambu-Goldstone Higgs, Lect. Notes Phys. 913 (2016) pp.1-316, [arXiv:1506.01961].

[34] R. Barbieri, B. Bellazzini, V. S. Rychkov, and A. Varagnolo, The Higgs boson from an extended symmetry, Phys. Rev. D76 (2007) 115008, [arXiv:0706.0432].

[35] D. B. Kaplan, Flavor at SSC energies: A New mechanism for dynamically generated fermion masses, Nucl. Phys. B365 (1991) 259-278.

[36] S. L. Glashow, Partial Symmetries of Weak Interactions, Nucl. Phys. 22 (1961) 579-588.

[37] M. Gell-Mann, A Schematic Model of Baryons and Mesons, Phys. Lett. 8 (1964) 214-215.

[38] G. Zweig, An SU(3) model for strong interaction symmetry and its breaking. Version 2, in DEVELOPMENTS IN THE QUARK THEORY OF HADRONS. VOL. 1. 1964 - 1978 (D. Lichtenberg and S. P. Rosen, eds.), pp. 22-101. 1964.

[39] S. Weinberg, A Model of Leptons, Phys. Rev. Lett. 19 (1967) 1264-1266.

[40] A. Salam, Weak and Electromagnetic Interactions, Conf. Proc. C680519 (1968) 367-377.

[41] Particle Data Group Collaboration, C. Patrignani et. al., Review of Particle Physics, Chin. Phys. C40 (2016), no. 10100001.

[42] C.-N. Yang and R. L. Mills, Conservation of Isotopic Spin and Isotopic Gauge Invariance, Phys. Rev. 96 (1954) 191-195.

[43] Y. Nambu, Quasiparticles and Gauge Invariance in the Theory of Superconductivity, Phys. Rev. 117 (1960) 648-663.

[44] J. Goldstone, Field Theories with Superconductor Solutions, Nuovo Cim. 19 (1961) 154-164. 
[45] J. Goldstone, A. Salam, and S. Weinberg, Broken Symmetries, Phys. Rev. 127 (1962) 965-970.

[46] V. L. Ginzburg and L. D. Landau, On the Theory of Superconductivity, Zh. Eksp. Teor. Fiz. 20 (1950) 1064-1082.

[47] P. W. Anderson, Plasmons, Gauge Invariance, and Mass, Phys. Rev. 130 (1963) 439-442.

[48] J. S. Schwinger, Gauge Invariance and Mass, Phys. Rev. 125 (1962) 397-398.

[49] J. S. Schwinger, Gauge Invariance and Mass. 2., Phys. Rev. 128 (1962) $2425-2429$.

[50] E. C. G. Stueckelberg, Die Wechselwirkungskräfte in der Elektrodynamik und in der Feldtheorie der Kräfte, Helv. Phys. Acta. 11 (1938) 225.

[51] LHC Higgs Cross Section Working Group Collaboration, S. Dittmaier et. al., Handbook of LHC Higgs Cross Sections: 1. Inclusive Observables, arXiv: 1101.0593.

[52] S. Dittmaier et. al., Handbook of LHC Higgs Cross Sections: 2. Differential Distributions, arXiv: 1201.3084.

[53] LHC Higgs Cross Section Working Group Collaboration, J. R. Andersen et. al., Handbook of LHC Higgs Cross Sections: 3. Higgs Properties, arXiv:1307.1347.

[54] LHC Higgs Cross Section Working Group Collaboration, D. de Florian et. al., Handbook of LHC Higgs Cross Sections: 4. Deciphering the Nature of the Higgs Sector, arXiv: 1610.07922.

[55] ATLAS Collaboration, G. Aad et. al., Evidence for the spin-0 nature of the Higgs boson using ATLAS data, Phys. Lett. B726 (2013) 120-144, [arXiv:1307.1432].

[56] CMS Collaboration, S. Chatrchyan et. al., Study of the Mass and Spin-Parity of the Higgs Boson Candidate Via Its Decays to Z Boson Pairs, Phys. Rev. Lett. 110 (2013), no. 8 081803, [arXiv:1212.6639].

[57] L. D. Landau, On the angular momentum of a system of two photons, Dokl. Akad. Nauk Ser. Fiz. 60 (1948), no. 2 207-209.

[58] C.-N. Yang, Selection Rules for the Dematerialization of a Particle Into Two Photons, Phys. Rev. 77 (1950) 242-245.

[59] ATLAS Collaboration, G. Aad et. al., Study of the spin and parity of the Higgs boson in diboson decays with the ATLAS detector, Eur. Phys. J. C75 (2015), no. 10 476, [arXiv:1506.05669]. [Erratum: Eur. Phys. J.C76,no.3,152(2016)].

[60] CMS Collaboration, V. Khachatryan et. al., Constraints on the spin-parity and anomalous $H V V$ couplings of the Higgs boson in proton collisions at 7 and 8 TeV, Phys. Rev. D92 (2015), no. 1 012004, [arXiv:1411.3441]. 
[61] ATLAS, CMS Collaboration, G. Aad et. al., Combined Measurement of the Higgs Boson Mass in pp Collisions at $\sqrt{s}=7$ and 8 TeV with the ATLAS and CMS Experiments, Phys. Rev. Lett. 114 (2015) 191803, [arXiv: 1503.07589].

[62] A. Denner, S. Heinemeyer, I. Puljak, D. Rebuzzi, and M. Spira, Standard Model Higgs-Boson Branching Ratios with Uncertainties, Eur. Phys. J. C71 (2011) 1753, [arXiv:1107.5909].

[63] ATLAS Collaboration, G. Aad et. al., Measurement of the Higgs boson mass from the $H \rightarrow \gamma \gamma$ and $H \rightarrow Z Z^{*} \rightarrow 4 \ell$ channels with the ATLAS detector using $25 \mathrm{fb}^{-1}$ of pp collision data, Phys. Rev. D90 (2014), no. 5 052004, [arXiv: 1406.3827].

[64] CMS Collaboration, V. Khachatryan et. al., Precise determination of the mass of the Higgs boson and tests of compatibility of its couplings with the standard model predictions using proton collisions at 7 and $8 \mathrm{TeV}$, Eur. Phys. J. C75 (2015), no. 5 212, [arXiv:1412.8662].

[65] ATLAS Collaboration, G. Aad et. al., Constraints on the off-shell Higgs boson signal strength in the high-mass $Z Z$ and $W W$ final states with the ATLAS detector, Eur. Phys. J. C75 (2015), no. 7 335, [arXiv:1503.01060].

[66] CMS Collaboration, V. Khachatryan et. al., Limits on the Higgs boson lifetime and width from its decay to four charged leptons, Phys. Rev. D92 (2015), no. 7 072010, [arXiv:1507.06656].

[67] ATLAS, CMS Collaboration, G. Aad et. al., Measurements of the Higgs boson production and decay rates and constraints on its couplings from a combined ATLAS and CMS analysis of the LHC pp collision data at $\sqrt{s}=7$ and $8 \mathrm{TeV}$, JHEP 08 (2016) 045, [arXiv: 1606.02266].

[68] LHC Higgs Cross Section Working Group Collaboration, A. David, A. Denner, M. Duehrssen, M. Grazzini, C. Grojean, G. Passarino, M. Schumacher, M. Spira, G. Weiglein, and M. Zanetti, LHC HXSWG interim recommendations to explore the coupling structure of a Higgs-like particle, arXiv:1209.0040.

[69] J. Ellis and T. You, Updated Global Analysis of Higgs Couplings, JHEP 06 (2013) 103, [arXiv: 1303.3879].

[70] C. Mariotti and G. Passarino, Higgs boson couplings: measurements and theoretical interpretation, Int. J. Mod. Phys. A32 (2017), no. 041730003 , [arXiv: 1612.00269].

[71] H. Arason, D. J. Castano, B. Keszthelyi, S. Mikaelian, E. J. Piard, P. Ramond, and B. D. Wright, Renormalization group study of the standard model and its extensions. 1. The Standard model, Phys. Rev. D46 (1992) 3945-3965.

[72] D. Buttazzo, G. Degrassi, P. P. Giardino, G. F. Giudice, F. Sala, A. Salvio, and A. Strumia, Investigating the near-criticality of the Higgs boson, JHEP 12 (2013) 089, [arXiv: 1307.3536]. 
[73] J. Elias-Miro, J. R. Espinosa, G. F. Giudice, G. Isidori, A. Riotto, and A. Strumia, Higgs mass implications on the stability of the electroweak vacuum, Phys. Lett. B709 (2012) 222-228, [arXiv:1112.3022].

[74] G. Degrassi, S. Di Vita, J. Elias-Miro, J. R. Espinosa, G. F. Giudice, G. Isidori, and A. Strumia, Higgs mass and vacuum stability in the Standard Model at NNLO, JHEP 08 (2012) 098, [arXiv:1205.6497].

[75] J. R. Espinosa, G. F. Giudice, E. Morgante, A. Riotto, L. Senatore, A. Strumia, and N. Tetradis, The cosmological Higgstory of the vacuum instability, JHEP 09 (2015) 174, [arXiv: 1505.04825].

[76] G. 't Hooft, Naturalness, chiral symmetry, and spontaneous chiral symmetry breaking, NATO Sci. Ser. B 59 (1980) 135-157.

[77] M. K. Gaillard and B. W. Lee, Rare Decay Modes of the K-Mesons in Gauge Theories, Phys. Rev. D10 (1974) 897.

[78] S. L. Glashow, J. Iliopoulos, and L. Maiani, Weak Interactions with Lepton-Hadron Symmetry, Phys. Rev. D2 (1970) 1285-1292.

[79] V. Agrawal, S. M. Barr, J. F. Donoghue, and D. Seckel, The Anthropic principle and the mass scale of the standard model, Phys. Rev. D57 (1998) 5480-5492, [hep-ph/9707380].

[80] P. W. Graham, D. E. Kaplan, and S. Rajendran, Cosmological Relaxation of the Electroweak Scale, Phys. Rev. Lett. 115 (2015), no. 22 221801, [arXiv: 1504.07551].

[81] L. F. Abbott, A Mechanism for Reducing the Value of the Cosmological Constant, Phys. Lett. B150 (1985) 427-430.

[82] R. S. Gupta, Z. Komargodski, G. Perez, and L. Ubaldi, Is the Relaxion an Axion?, JHEP 02 (2016) 166, [arXiv:1509.00047].

[83] S. Weinberg, Implications of Dynamical Symmetry Breaking, Phys. Rev. D13 (1976) 974-996.

[84] S. Weinberg, Implications of Dynamical Symmetry Breaking: An Addendum, Phys. Rev. D19 (1979) 1277-1280.

[85] L. Susskind, Dynamics of Spontaneous Symmetry Breaking in the Weinberg-Salam Theory, Phys.Rev. D20 (1979) 2619-2625.

[86] S. Dimopoulos and L. Susskind, Mass Without Scalars, Nucl.Phys. B155 (1979) 237-252.

[87] S. Dimopoulos and J. Preskill, Massless Composites With Massive Constituents, Nucl.Phys. B199 (1982) 206.

[88] C. Quigg and R. Shrock, Gedanken Worlds without Higgs: QCD-Induced Electroweak Symmetry Breaking, Phys. Rev. D79 (2009) 096002, [arXiv:0901.3958]. 
[89] R. Contino, The Higgs as a Composite Nambu-Goldstone Boson, arXiv: 1005.4269.

[90] G. 't Hooft, A Planar Diagram Theory for Strong Interactions, Nucl. Phys. B72 (1974) 461.

[91] G. 't Hooft, A Two-Dimensional Model for Mesons, Nucl. Phys. B75 (1974) 461-470.

[92] E. Witten, Baryons in the 1/n Expansion, Nucl. Phys. B160 (1979) 57-115.

[93] M. E. Peskin and T. Takeuchi, A New constraint on a strongly interacting Higgs sector, Phys.Rev.Lett. 65 (1990) 964-967.

[94] B. Holdom and J. Terning, Large corrections to electroweak parameters in technicolor theories, Phys. Lett. B247 (1990) 88-92.

[95] M. Golden and L. Randall, Radiative Corrections to Electroweak Parameters in Technicolor Theories, Nucl. Phys. B361 (1991) 3-23.

[96] D. B. Kaplan and H. Georgi, $S U(2) \times U(1)$ Breaking by Vacuum Misalignment, Phys.Lett. B136 (1984) 183.

[97] D. B. Kaplan, H. Georgi, and S. Dimopoulos, Composite Higgs Scalars, Phys.Lett. B136 (1984) 187.

[98] H. Georgi, D. B. Kaplan, and P. Galison, Calculation of the Composite Higgs Mass, Phys.Lett. B143 (1984) 152.

[99] M. J. Dugan, H. Georgi, and D. B. Kaplan, Anatomy of a Composite Higgs Model, Nucl.Phys. B254 (1985) 299.

[100] A. Manohar and H. Georgi, Chiral Quarks and the Nonrelativistic Quark Model, Nucl.Phys. B234 (1984) 189.

[101] V. Sanz and J. Setford, Composite Higgs models after Run2, arXiv: 1703.10190.

[102] R. Contino, L. Da Rold, and A. Pomarol, Light custodians in natural composite Higgs models, Phys.Rev. D75 (2007) 055014, [hep-ph/0612048].

[103] B. Gripaios, A. Pomarol, F. Riva, and J. Serra, Beyond the Minimal Composite Higgs Model, JHEP 0904 (2009) 070, [arXiv:0902.1483].

[104] G. Ferretti and D. Karateev, Fermionic UV completions of Composite Higgs models, JHEP 03 (2014) 077, [arXiv:1312.5330].

[105] G. Cacciapaglia and F. Sannino, Fundamental Composite (Goldstone) Higgs Dynamics, JHEP 04 (2014) 111, [arXiv: 1402.0233].

[106] G. Cacciapaglia and F. Sannino, An Ultraviolet Chiral Theory of the Top for the Fundamental Composite (Goldstone) Higgs, Phys. Lett. B755 (2016) 328-331, [arXiv:1508.00016]. 
[107] R. Barbieri, D. Buttazzo, F. Sala, D. M. Straub, and A. Tesi, A $125 \mathrm{GeV}$ composite Higgs boson versus flavour and electroweak precision tests, JHEP 05 (2013) 069, [arXiv:1211.5085].

[108] A. Pomarol and F. Riva, The Composite Higgs and Light Resonance Connection, JHEP 08 (2012) 135, [arXiv: 1205.6434].

[109] G. Panico, M. Redi, A. Tesi, and A. Wulzer, On the Tuning and the Mass of the Composite Higgs, JHEP 03 (2013) 051, [arXiv: 1210.7114].

[110] C. Grojean, O. Matsedonskyi, and G. Panico, Light top partners and precision physics, JHEP 1310 (2013) 160, [arXiv:1306.4655].

[111] K. Agashe, R. Contino, L. Da Rold, and A. Pomarol, A Custodial symmetry for Zbb, Phys. Lett. B641 (2006) 62-66, [hep-ph/0605341].

[112] L. Randall and R. Sundrum, A Large mass hierarchy from a small extra dimension, Phys. Rev. Lett. 83 (1999) 3370-3373, [hep-ph/9905221].

[113] R. Contino, Y. Nomura, and A. Pomarol, Higgs as a holographic pseudoGoldstone boson, Nucl. Phys. B671 (2003) 148-174, [hep-ph/0306259].

[114] D. Pappadopulo, A. Thamm, and R. Torre, A minimally tuned composite Higgs model from an extra dimension, JHEP 07 (2013) 058, [arXiv:1303.3062].

[115] N. Arkani-Hamed, A. G. Cohen, and H. Georgi, Electroweak symmetry breaking from dimensional deconstruction, Phys.Lett. B513 (2001) 232-240, [hep-ph/0105239].

[116] N. Arkani-Hamed, A. G. Cohen, E. Katz, A. E. Nelson, T. Gregoire, and J. G. Wacker, The Minimal moose for a little Higgs, JHEP 08 (2002) 021, [hep-ph/0206020].

[117] N. Arkani-Hamed, A. G. Cohen, E. Katz, and A. E. Nelson, The Littlest Higgs, JHEP 07 (2002) 034, [hep-ph/0206021].

[118] H. Georgi, The Higgs as a pseudo-Goldstone boson, Comptes Rendus Physique 8 (2007) 1029-1047.

[119] S. R. Coleman, J. Wess, and B. Zumino, Structure of phenomenological Lagrangians. 1., Phys. Rev. 177 (1969) 2239-2247.

[120] C. G. Callan, Jr., S. R. Coleman, J. Wess, and B. Zumino, Structure of phenomenological Lagrangians. 2., Phys. Rev. 177 (1969) 2247-2250.

[121] S. Weinberg, Phenomenological Lagrangians, Physica A96 (1979) 327-340.

[122] T. Appelquist, M. J. Bowick, E. Cohler, and A. I. Hauser, The Breaking of Isospin Symmetry in Theories With a Dynamical Higgs Mechanism, Phys.Rev. D31 (1985) 1676. 
[123] T. Appelquist and G.-H. Wu, The Electroweak chiral Lagrangian and new precision measurements, Phys.Rev. D48 (1993) 3235-3241, [hep-ph/9304240].

[124] A. Azatov, R. Contino, and J. Galloway, Model-Independent Bounds on a Light Higgs, JHEP 1204 (2012) 127, [arXiv:1202.3415].

[125] R. Alonso, M. Gavela, L. Merlo, S. Rigolin, and J. Yepes, The Effective Chiral Lagrangian for a Light Dynamical "Higgs Particle", Phys.Lett. B722 (2013) 330-335, [arXiv:1212.3305].

[126] G. Buchalla, O. Cata, and C. Krause, Complete Electroweak Chiral Lagrangian with a Light Higgs at NLO, Nucl.Phys. B880 (2014) 552-573, [arXiv:1307.5017].

[127] I. Brivio, J. Gonzalez-Fraile, M. C. Gonzalez-Garcia, and L. Merlo, The complete HEFT Lagrangian after the LHC Run I, Eur. Phys. J. C76 (2016), no. 7 416, [arXiv: 1604.06801].

[128] G. Giudice, C. Grojean, A. Pomarol, and R. Rattazzi, The Strongly-Interacting Light Higgs, JHEP 0706 (2007) 045, [hep-ph/0703164].

[129] A. G. Cohen, D. B. Kaplan, and A. E. Nelson, Counting 4 pis in strongly coupled supersymmetry, Phys. Lett. B412 (1997) 301-308, [hep-ph/9706275].

[130] M. A. Luty, Naive dimensional analysis and supersymmetry, Phys. Rev. D57 (1998) 1531-1538, [hep-ph/9706235].

[131] E. E. Jenkins, A. V. Manohar, and M. Trott, Naive Dimensional Analysis Counting of Gauge Theory Amplitudes and Anomalous Dimensions, Phys.Lett. B726 (2013) 697-702, [arXiv: 1309.0819].

[132] G. Buchalla, O. Cata, and C. Krause, On the Power Counting in Effective Field Theories, Phys.Lett. B731 (2014) 80-86, [arXiv:1312.5624].

[133] G. Buchalla, O. Cata, and C. Krause, A Systematic Approach to the SILH Lagrangian, Nucl. Phys. B894 (2015) 602-620, [arXiv:1412.6356].

[134] G. Buchalla, O. Cata, A. Celis, and C. Krause, Comment on "Analysis of General Power Counting Rules in Effective Field Theory", arXiv:1603.03062.

[135] B. M. Gavela, E. E. Jenkins, A. V. Manohar, and L. Merlo, Analysis of General Power Counting Rules in Effective Field Theory, Eur. Phys. J. C76 (2016), no. 9 485, [arXiv:1601.07551].

[136] W. Buchmuller and D. Wyler, Effective Lagrangian Analysis of New Interactions and Flavor Conservation, Nucl.Phys. B268 (1986) 621-653.

[137] B. Grzadkowski, M. Iskrzynski, M. Misiak, and J. Rosiek, Dimension-Six Terms in the Standard Model Lagrangian, JHEP 1010 (2010) 085, [arXiv:1008.4884]. 
[138] K. Hagiwara, S. Ishihara, R. Szalapski, and D. Zeppenfeld, Low-energy effects of new interactions in the electroweak boson sector, Phys.Rev. D48 (1993) 2182-2203.

[139] K. Hagiwara, T. Hatsukano, S. Ishihara, and R. Szalapski, Probing nonstandard bosonic interactions via $W$ boson pair production at lepton colliders, Nucl.Phys. B496 (1997) 66-102, [hep-ph/9612268].

[140] I. Brivio, T. Corbett, O. J. P. Éboli, M. B. Gavela, J. Gonzalez-Fraile, M. C. Gonzalez-Garcia, L. Merlo, and S. Rigolin, Disentangling a dynamical Higgs, JHEP 03 (2014) 024, [arXiv: 1311.1823].

[141] J. Gasser and H. Leutwyler, Chiral Perturbation Theory to One Loop, Annals Phys. 158 (1984) 142.

[142] M. B. Gavela, K. Kanshin, P. A. N. Machado, and S. Saa, On the renormalization of the electroweak chiral Lagrangian with a Higgs, JHEP 03 (2015) 043, [arXiv:1409.1571].

[143] M. S. Chanowitz and M. K. Gaillard, The TeV Physics of Strongly Interacting W's and Z's, Nucl. Phys. B261 (1985) 379-431.

[144] G. J. Gounaris, R. Kogerler, and H. Neufeld, Relationship Between Longitudinally Polarized Vector Bosons and their Unphysical Scalar Partners, Phys. Rev. D34 (1986) 3257.

[145] M. S. Chanowitz, M. Golden, and H. Georgi, Low-Energy Theorems for Strongly Interacting W's and Z's, Phys.Rev. D36 (1987) 1490.

[146] T. Appelquist and C. W. Bernard, The Nonlinear $\sigma$ Model in the Loop Expansion, Phys.Rev. D23 (1981) 425.

[147] S. Weinberg, Nonlinear realizations of chiral symmetry, Phys.Rev. 166 (1968) 1568-1577.

[148] R. Alonso, E. E. Jenkins, and A. V. Manohar, A Geometric Formulation of Higgs Effective Field Theory: Measuring the Curvature of Scalar Field Space, Phys. Lett. B754 (2016) 335-342, [arXiv:1511.00724].

[149] R. Alonso, E. E. Jenkins, and A. V. Manohar, Geometry of the Scalar Sector, JHEP 08 (2016) 101, [arXiv: 1605.03602].

[150] R. Alonso, E. E. Jenkins, and A. V. Manohar, Sigma Models with Negative Curvature, Phys. Lett. B756 (2016) 358-364, [arXiv:1602.00706].

[151] J. Charap, Closed-loop calculations using a chiral-invariant lagrangian, Phys.Rev. D2 (1970) 1554-1561.

[152] I. Gerstein, R. Jackiw, S. Weinberg, and B. Lee, Chiral loops, Phys.Rev. D3 (1971) 2486-2492.

[153] R. Mertig, M. Bohm, and A. Denner, FEYN CALC: Computer algebraic calculation of Feynman amplitudes, Comput. Phys. Commun. 64 (1991) 345-359. 
[154] A. Alloul, N. D. Christensen, C. Degrande, C. Duhr, and B. Fuks, FeynRules 2.0 - A complete toolbox for tree-level phenomenology, Comput. Phys.

Commun. 185 (2014) 2250-2300, [arXiv:1310.1921].

[155] J. Kublbeck, M. Bohm, and A. Denner, Feyn Arts: Computer Algebraic Generation of Feynman Graphs and Amplitudes, Comput. Phys. Commun. 60 (1990) 165-180.

[156] T. Hahn, Generating Feynman diagrams and amplitudes with FeynArts 3, Comput. Phys. Commun. 140 (2001) 418-431, [hep-ph/0012260].

[157] T. Hahn and M. Perez-Victoria, Automatized one loop calculations in four-dimensions and D-dimensions, Comput. Phys. Commun. 118 (1999) 153-165, [hep-ph/9807565].

[158] B. de Wit and M. T. Grisaru, On-shell Counterterms and Nonlinear Invariances, Phys.Rev. D20 (1979) 2082.

[159] J. Honerkamp, Chiral multiloops, Nucl.Phys. B36 (1972) 130-140.

[160] D. Kazakov, V. Pervushin, and S. Pushkin, Invariant Renormalization for the Field Theories with Nonlinear Symmetry, Teor.Mat.Fiz. 31 (1977) 169-176.

[161] D. Kazakov, V. Pervushin, and S. Pushkin, An Invariant Renormalization Method for Nonlinear Realizations of the Dynamical Symmetries, Theor.Math.Phys. 31 (1977) 389.

[162] M. Ostrogradsky, Mémoire sur les équations différentielles relatives an problème des isopérimétres, .

[163] C. Grosse-Knetter, Effective Lagrangians with higher derivatives and equations of motion, Phys.Rev. D49 (1994) 6709-6719, [hep-ph/9306321].

[164] S. Scherer and H. W. Fearing, Field transformations and the classical equation of motion in chiral perturbation theory, Phys. Rev. D52 (1995) 6445-6450, [hep-ph/9408298].

[165] C. Arzt, Reduced effective Lagrangians, Phys. Lett. B342 (1995) 189-195, [hep-ph/9304230].

[166] J. Bijnens, G. Colangelo, and G. Ecker, The Mesonic chiral Lagrangian of order $p^{6}$, JHEP 02 (1999) 020, [hep-ph/9902437].

[167] R. Alonso, K. Kanshin, and S. Saa, One loop renormalization of Higgs Effective Field Theory, . In preparation.

[168] M. K. Gaillard, The Effective One Loop Lagrangian With Derivative Couplings, Nucl. Phys. B268 (1986) 669-692.

[169] O. Cheyette, Effective Action for the Standard Model With Large Higgs Mass, Nucl. Phys. B297 (1988) 183-204.

[170] B. Henning, X. Lu, and H. Murayama, How to use the Standard Model effective field theory, JHEP 01 (2016) 023, [arXiv:1412.1837]. 
[171] A. Drozd, J. Ellis, J. Quevillon, and T. You, The Universal One-Loop Effective Action, JHEP 03 (2016) 180, [arXiv:1512.03003].

[172] B. Henning, X. Lu, and H. Murayama, One-loop Matching and Running with Covariant Derivative Expansion, arXiv:1604.01019.

[173] F. del Aguila, Z. Kunszt, and J. Santiago, One-loop effective lagrangians after matching, Eur. Phys. J. C76 (2016), no. 5 244, [arXiv:1602.00126].

[174] J. Fuentes-Martin, J. Portoles, and P. Ruiz-Femenia, Integrating out heavy particles with functional methods: a simplified framework, JHEP 09 (2016) 156, [arXiv: 1607.02142].

[175] L. F. Abbott, Introduction to the Background Field Method, Acta Phys. Polon. B13 (1982) 33.

[176] M. Srednicki, Quantum field theory. Cambridge University Press, 2007.

[177] M. E. Peskin and T. Takeuchi, Estimation of oblique electroweak corrections, Phys. Rev. D46 (1992) 381-409.

[178] C. Cheung and C.-H. Shen, Nonrenormalization Theorems without Supersymmetry, Phys. Rev. Lett. 115 (2015), no. 7 071601, [arXiv: 1505.01844].

[179] R. Alonso, E. E. Jenkins, and A. V. Manohar, Holomorphy without Supersymmetry in the Standard Model Effective Field Theory, Phys. Lett. B739 (2014) 95-98, [arXiv: 1409.0868].

[180] F. Feruglio, B. Gavela, K. Kanshin, P. A. N. Machado, S. Rigolin, and S. Saa, The minimal linear sigma model for the Goldstone Higgs, JHEP 06 (2016) 038, [arXiv:1603.05668].

[181] D. Marzocca, M. Serone, and J. Shu, General Composite Higgs Models, JHEP 1208 (2012) 013, [arXiv:1205.0770].

[182] M. Redi and A. Tesi, Implications of a Light Higgs in Composite Models, JHEP 10 (2012) 166, [arXiv: 1205.0232].

[183] A. Carmona and F. Goertz, A naturally light Higgs without light Top Partners, JHEP 05 (2015) 002, [arXiv: 1410.8555].

[184] G. von Gersdorff, E. Pontón, and R. Rosenfeld, The Dynamical Composite Higgs, JHEP 06 (2015) 119, [arXiv:1502.07340].

[185] L. J. Dixon and Y. Li, Bounding the Higgs Boson Width Through Interferometry, Phys. Rev. Lett. 111 (2013) 111802, [arXiv:1305. 3854].

[186] J. F. Gunion, H. E. Haber, G. L. Kane, and S. Dawson, The Higgs Hunter's Guide, Front. Phys. 80 (2000) 1-448.

[187] S. R. Coleman and E. J. Weinberg, Radiative Corrections as the Origin of Spontaneous Symmetry Breaking, Phys. Rev. D7 (1973) 1888-1910.

[188] D. Ghosh, M. Salvarezza, and F. Senia, Extending the Analysis of Electroweak Precision Constraints in Composite Higgs Models, arXiv:1511.08235. 
[189] H. Gertov, A. Meroni, E. Molinaro, and F. Sannino, Theory and phenomenology of the elementary Goldstone Higgs boson, Phys. Rev. D92 (2015), no. 9 095003, [arXiv:1507.06666].

[190] G. Altarelli and R. Barbieri, Vacuum polarization effects of new physics on electroweak processes, Phys. Lett. B253 (1991) 161-167.

[191] M. Ciuchini, E. Franco, S. Mishima, M. Pierini, L. Reina, and L. Silvestrini, Update of the electroweak precision fit, interplay with Higgs-boson signal strengths and model-independent constraints on new physics, in International Conference on High Energy Physics 2014 (ICHEP 2014) Valencia, Spain, July 2-9, 2014, 2014. arXiv:1410.6940.

[192] V. A. Novikov, L. B. Okun, and M. I. Vysotsky, On the Electroweak one loop corrections, Nucl. Phys. B397 (1993) 35-83.

[193] A. Orgogozo and S. Rychkov, The S parameter for a Light Composite Higgs: a Dispersion Relation Approach, JHEP 06 (2013) 014, [arXiv:1211.5543].

[194] H. E. Haber and D. O'Neil, Basis-independent methods for the two-Higgs-doublet model III: The CP-conserving limit, custodial symmetry, and the oblique parameters S, T, U, Phys. Rev. D83 (2011) 055017, [arXiv: 1011.6188].

[195] C. Anastasiou, E. Furlan, and J. Santiago, Realistic Composite Higgs Models, Phys. Rev. D79 (2009) 075003, [arXiv:0901.2117].

[196] L. Lavoura and J. P. Silva, The Oblique corrections from vector - like singlet and doublet quarks, Phys. Rev. D47 (1993) 2046-2057.

[197] ATLAS Collaboration, G. Aad et. al., Search for production of vector-like quark pairs and of four top quarks in the lepton-plus-jets final state in $p p$ collisions at $\sqrt{s}=8 \mathrm{TeV}$ with the ATLAS detector, JHEP 08 (2015) 105, [arXiv:1505.04306].

[198] CMS Collaboration, Search for top quark partners with charge 5/3 at $\sqrt{s}=13 \mathrm{TeV}$.

[199] S. Dawson and E. Furlan, A Higgs Conundrum with Vector Fermions, Phys. Rev. D86 (2012) 015021, [arXiv:1205.4733].

[200] S. Fichet, G. von Gersdorff, E. PontÂşn, and R. Rosenfeld, The Global Higgs as a Signal for Compositeness at the LHC, JHEP 01 (2017) 012, [arXiv:1608.01995].

[201] ATLAS Collaboration, G. Aad et. al., Search for Scalar Diphoton Resonances in the Mass Range 65-600 GeV with the ATLAS Detector in pp Collision Data at $\sqrt{s}=8 \mathrm{TeV}$, Phys. Rev. Lett. 113 (2014), no. 17 171801, [arXiv: 1407.6583].

[202] CMS Collaboration, V. Khachatryan et. al., Search for diphoton resonances in the mass range from 150 to $850 \mathrm{GeV}$ in pp collisions at $\sqrt{s}=8 \mathrm{TeV}$, Phys. Lett. B750 (2015) 494-519, [arXiv:1506.02301]. 
[203] ATLAS Collaboration, G. Aad et. al., Search for a high-mass Higgs boson decaying to a $W$ boson pair in pp collisions at $\sqrt{s}=8$ TeV with the ATLAS detector, JHEP 01 (2016) 032, [arXiv:1509.00389].

[204] ATLAS Collaboration, G. Aad et. al., Search for an additional, heavy Higgs boson in the $H \rightarrow Z Z$ decay channel at $\sqrt{s}=8 \mathrm{TeV}$ in pp collision data with the ATLAS detector, Eur. Phys. J. C76 (2016), no. 1 45, [arXiv: 1507.05930].

[205] CMS Collaboration, Properties of the Higgs-like boson in the decay $H$ to ZZ to $4 \mathrm{l}$ in $p p$ collisions at sqrt $s=7$ and $8 \mathrm{TeV}$.

[206] CMS Collaboration, Update on the search for the standard model Higgs boson in pp collisions at the $\mathrm{LHC}$ decaying to $W+W$ in the fully leptonic final state, .

[207] CMS Collaboration, V. Khachatryan et. al., Search for resonant pair production of Higgs bosons decaying to two bottom quark-antiquark pairs in proton-proton collisions at $8 \mathrm{TeV}$, Phys. Lett. B749 (2015) 560-582, [arXiv: 1503.04114].

[208] ATLAS Collaboration, G. Aad et. al., Searches for Higgs boson pair production in the $h h \rightarrow b b \tau \tau, \gamma \gamma W W^{*}, \gamma \gamma b b, b b b b$ channels with the ATLAS detector, Phys. Rev. D92 (2015) 092004, [arXiv:1509.04670].

[209] ATLAS, CMS Collaboration, A. Holzner, Beyond standard model Higgs physics: prospects for the High Luminosity LHC, arXiv: 1411.0322.

[210] V. Martín Lozano, J. M. Moreno, and C. B. Park, Resonant Higgs boson pair production in the $h h \rightarrow b \bar{b} W W \rightarrow b \bar{b} \ell^{+} \nu \ell^{-} \bar{\nu}$ decay channel, JHEP 08 (2015) 004, [arXiv: 1501.03799].

[211] M. B. Gavela, K. Kanshin, P. A. N. Machado, and S. Saa, The linear-non-linear frontier for the Goldstone Higgs, Eur. Phys. J. C76 (2016), no. 12 690, [arXiv: 1610.08083].

[212] G. Buchalla, O. Cata, A. Celis, and C. Krause, Standard Model Extended by a Heavy Singlet: Linear vs. Nonlinear EFT, Nucl. Phys. B917 (2017) 209-233, [arXiv: 1608.03564].

[213] R. Alonso, I. Brivio, B. Gavela, L. Merlo, and S. Rigolin, Sigma Decomposition, JHEP 12 (2014) 034, [arXiv:1409.1589].

[214] ATLAS Collaboration, G. Aad et. al., Evidence for Electroweak Production of $W^{ \pm} W^{ \pm} j j$ in pp Collisions at $\sqrt{s}=8 \mathrm{TeV}$ with the ATLAS Detector, Phys. Rev. Lett. 113 (2014), no. 14 141803, [arXiv:1405.6241].

[215] ATLAS Collaboration, G. Aad et. al., Measurements of $W^{ \pm} Z$ production cross sections in pp collisions at $\sqrt{s}=8 \mathrm{TeV}$ with the ATLAS detector and limits on anomalous gauge boson self-couplings, Phys. Rev. D93 (2016), no. 9 092004, [arXiv:1603.02151]. 
[216] M. B. Gavela, P. Hernandez, J. Orloff, and O. Pene, Standard model CP violation and baryon asymmetry, Mod. Phys. Lett. A9 (1994) 795-810, [hep-ph/9312215].

[217] M. B. Gavela, M. Lozano, J. Orloff, and O. Pene, Standard model CP violation and baryon asymmetry. Part 1: Zero temperature, Nucl. Phys. B430 (1994) 345-381, [hep-ph/9406288].

[218] M. B. Gavela, P. Hernandez, J. Orloff, O. Pene, and C. Quimbay, Standard model CP violation and baryon asymmetry. Part 2: Finite temperature, Nucl. Phys. B430 (1994) 382-426, [hep-ph/9406289].

[219] L. Merlo, S. Saa, and M. Sacristán-Barbero, Baryon Non-Invariant Couplings in Higgs Effective Field Theory, Eur. Phys. J. C77 (2017), no. 3 185, [arXiv: 1612.04832].

[220] S. Weinberg, Baryon and Lepton Nonconserving Processes, Phys. Rev. Lett. 43 (1979) 1566-1570.

[221] F. Wilczek and A. Zee, Operator Analysis of Nucleon Decay, Phys. Rev. Lett. 43 (1979) 1571-1573.

[222] H. A. Weldon and A. Zee, Operator Analysis of New Physics, Nucl. Phys. B173 (1980) 269-290.

[223] L. F. Abbott and M. B. Wise, The Effective Hamiltonian for Nucleon Decay, Phys. Rev. D22 (1980) 2208.

[224] R. Alonso, H.-M. Chang, E. E. Jenkins, A. V. Manohar, and B. Shotwell, Renormalization group evolution of dimension-six baryon number violating operators, Phys. Lett. B734 (2014) 302-307, [arXiv:1405.0486].

[225] Super-Kamiokande Collaboration, H. Nishino et. al., Search for Proton Decay via $\pi \rightarrow e^{+} \pi^{0}$ and $p \rightarrow \mu^{+} \pi^{0}$ in a Large Water Cherenkov Detector, Phys. Rev. Lett. 102 (2009) 141801, [arXiv:0903.0676].

[226] Super-Kamiokande Collaboration, H. Nishino et. al., Search for Nucleon Decay into Charged Anti-lepton plus Meson in Super-Kamiokande I and II, Phys. Rev. D85 (2012) 112001, [arXiv:1203.4030].

[227] Super-Kamiokande Collaboration, K. Abe et. al., Search for proton decay via $p \rightarrow e^{+} \pi^{0}$ and $p \rightarrow \mu^{+} \pi^{0}$ in 0.31 megaton.years exposure of the Super-Kamiokande water Cherenkov detector, Phys. Rev. D95 (2017), no. 1 012004, [arXiv:1610.03597].

[228] R. S. Chivukula and H. Georgi, Composite Technicolor Standard Model, Phys.Lett. B188 (1987) 99.

[229] G. D'Ambrosio, G. Giudice, G. Isidori, and A. Strumia, Minimal flavor violation: An Effective field theory approach, Nucl.Phys. B645 (2002) 155-187, [hep-ph/0207036]. 
[230] V. Cirigliano, B. Grinstein, G. Isidori, and M. B. Wise, Minimal flavor violation in the lepton sector, Nucl.Phys. B728 (2005) 121-134, [hep-ph/0507001].

[231] S. Davidson and F. Palorini, Various definitions of Minimal Flavour Violation for Leptons, Phys.Lett. B642 (2006) 72-80, [hep-ph/0607329].

[232] R. Alonso, G. Isidori, L. Merlo, L. A. Munoz, and E. Nardi, Minimal flavour violation extensions of the seesaw, JHEP 1106 (2011) 037, [arXiv: 1103.5461].

[233] R. Alonso, M. Gavela, L. Merlo, and S. Rigolin, On the scalar potential of minimal flavour violation, JHEP 1107 (2011) 012, [arXiv:1103.2915].

[234] R. Alonso, M. Gavela, D. Hernandez, and L. Merlo, On the Potential of Leptonic Minimal Flavour Violation, Phys.Lett. B715 (2012) 194-198, [arXiv:1206.3167].

[235] R. Alonso, M. B. Gavela, D. Hernandez, L. Merlo, and S. Rigolin, Leptonic Dynamical Yukawa Couplings, JHEP 08 (2013) 069, [arXiv:1306.5922].

[236] R. Alonso, M. B. Gavela, G. Isidori, and L. Maiani, Neutrino Mixing and Masses from a Minimum Principle, JHEP 11 (2013) 187, [arXiv:1306.5927].

[237] B. Grinstein, M. Redi, and G. Villadoro, Low Scale Flavor Gauge Symmetries, JHEP 1011 (2010) 067, [arXiv:1009.2049].

[238] T. Feldmann, See-Saw Masses for Quarks and Leptons in SU(5), JHEP 04 (2011) 043, [arXiv:1010.2116].

[239] D. Guadagnoli, R. N. Mohapatra, and I. Sung, Gauged Flavor Group with Left-Right Symmetry, JHEP 04 (2011) 093, [arXiv:1103.4170].

[240] A. J. Buras, L. Merlo, and E. Stamou, The Impact of Flavour Changing Neutral Gauge Bosons on $\bar{B} \rightarrow X_{s} \gamma$, JHEP 08 (2011) 124, [arXiv:1105.5146].

[241] A. J. Buras, M. V. Carlucci, L. Merlo, and E. Stamou, Phenomenology of a Gauged SU $(3)^{3}$ Flavour Model, JHEP 03 (2012) 088, [arXiv:1112.4477].

[242] T. Feldmann, C. Luhn, and P. Moch, Lepton-flavour violation in a Pati-Salam model with gauged flavour symmetry, JHEP 11 (2016) 078, [arXiv: 1608.04124].

[243] R. Alonso, E. Fernandez Martinez, M. B. Gavela, B. Grinstein, L. Merlo, and P. Quilez, Gauged Lepton Flavour, JHEP 12 (2016) 119, [arXiv: 1609.05902].

[244] J. C. Pati and A. Salam, Lepton Number as the Fourth Color, Phys. Rev. D10 (1974) 275-289. [Erratum: Phys. Rev.D11,703(1975)].

[245] R. N. Mohapatra and J. C. Pati, A Natural Left-Right Symmetry, Phys. Rev. D11 (1975) 2558. 
[246] A. Kobach, Baryon Number, Lepton Number, and Operator Dimension in the Standard Model, Phys. Lett. B758 (2016) 455-457, [arXiv:1604.05726].

[247] S. Weinberg, Varieties of Baryon and Lepton Nonconservation, Phys. Rev. D22 (1980) 1694.

[248] J. Hirn and J. Stern, Lepton-number violation and right-handed neutrinos in Higgs-less effective theories, Phys. Rev. D73 (2006) 056001, [hep-ph/0504277].

[249] G. Buchalla and O. Cata, Effective Theory of a Dynamically Broken Electroweak Standard Model at NLO, JHEP 07 (2012) 101, [arXiv: 1203.6510].

[250] E. E. Jenkins and A. V. Manohar, Algebraic Structure of Lepton and Quark Flavor Invariants and CP Violation, JHEP 10 (2009) 094, [arXiv:0907.4763].

[251] A. Hanany, E. E. Jenkins, A. V. Manohar, and G. Torri, Hilbert Series for Flavor Invariants of the Standard Model, JHEP 03 (2011) 096, [arXiv:1010.3161].

[252] L. Lehman and A. Martin, Hilbert Series for Constructing Lagrangians: expanding the phenomenologist's toolbox, Phys. Rev. D91 (2015) 105014, [arXiv:1503.07537].

[253] B. Henning, X. Lu, T. Melia, and H. Murayama, Hilbert series and operator bases with derivatives in effective field theories, Commun. Math. Phys. 347 (2016), no. 2 363-388, [arXiv: 1507.07240].

[254] L. Lehman and A. Martin, Low-derivative operators of the Standard Model effective field theory via Hilbert series methods, JHEP 02 (2016) 081, [arXiv:1510.00372].

[255] B. Henning, X. Lu, T. Melia, and H. Murayama, 2, 84, 30, 993, 560, 15456, 11962, 261485, ...: Higher dimension operators in the SM EFT, arXiv:1512.03433.

[256] Y. Liao and X.-D. Ma, Operators up to Dimension Seven in Standard Model Effective Field Theory Extended with Sterile Neutrinos, arXiv:1612.04527. 